Centre for Petroleum, Fuels and Energy

The School of Mechanical Engineering

\title{
Sulphur Transformation during Pyrolysis of an Australian Lignite
}

by

Setyawati Yani

This thesis is presented for the Degree of

Doctor of Philosophy

in

Chemical Engineering

of

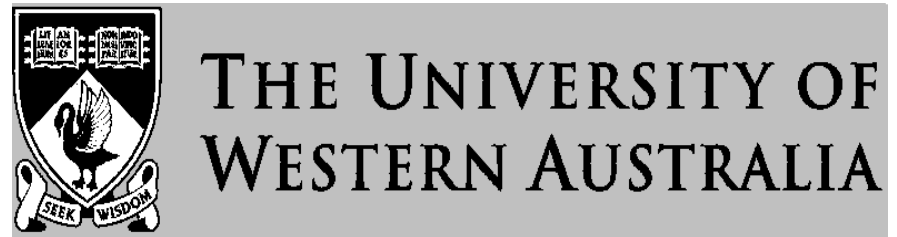

23 January 2009 


\section{DECLARATION}

To the best of my knowledge and belief this thesis contains no material previously published by any other person except where due acknowledgement has been made in the text. This thesis contains no material which has been accepted for the award of any other degree or diploma in any university.

I give consent to this copy of my thesis, when deposited in the University Library, being made available for photocopying and loan. 


\section{ACKNOWLEDGEMENTS}

\section{In the name of Allah, the Most Gracious, the Dispenser of Grace}

I would like to express my deep gratitude to Professor Dongke Zhang FTSE for his inspiration, support, advice and training throughout my candidature. He has been a role model for me from whom I have learned not only about academic matter but also about non academic subjects. It is my privilege to have worked with him.

Part of the thesis work was done in my previous centre, Centre for Fuels and Energy, Curtin University of Technology. Therefore, I would like to acknowledge colleagues in the Centre for the administrative or technical assistance, discussion and friendship: Ms. Angelina Rossiter, Ms. Tasneem Dawood, Mr. Zhezi Zhang, Mr. Cyril Kelly, Dr. Richard Gunawan, Dr. Ghia Hung Pham, and Dr. John Bromly. Special thanks also to Dr. Hamzah Fansuri and Dr. Nurul Widiastuti for friendship, encouragement and helping with XRD and FTIR analysis. To my fellow $\mathrm{PhD}$ candidate at UWA, Ms Meining Song, thank you for your support and providing data of pyrite mineral analysis.

I would also like to acknowledge Mr. Ian Sills of Curtin University of Technology and Mr. Ian Davies of CSIRO minerals for TGA-MS assistance, Associate Professor Ron Watkins and Dr. Dave Oldmeadow of EIGG for CS analysis assistance, Mrs Elaine Miller for SEM assistance and Dr. Lindsay Byrne of Chemistry Department UWA for NMR assistance.

A very big thank you goes to my beloved husband, Syamsuddin Yani, my energetic boy, Daffa, and my lovely daughter, Rayya, for their support, patient and understanding. To my big family in Indonesia, I would also like to say thank you for all your support. 
Acknowledgements

I also wish to acknowledge my home university, Moslem University of Indonesia, Makassar, Indonesia, for giving consent for myself to apply for Australian Development Scholarship and undertake this PhD study. Last but not least to Australian Development Scholarship, thank you very much for providing the scholarship. I trust the experience and knowledge I have gained during my $\mathrm{PhD}$ study will be beneficial to both Indonesia and Australia. 


\section{EXECUTIVE SUMMARY}

Australia has the largest economical lignite resources in the world. However, the utilisation of lignite is faced with a number of technical, economical and environmental problems due to its high moisture content and some of the deposits containing high sulphur and high inorganic matter. During pyrolysis, the first step of any thermochemical conversion processes of coal, some of the sulphur in lignite evolves as sulphur-bearing volatiles while others are retained in the solid phase of the lignite char. The present research aims to study the sulphur transformation during pyrolysis of Australian lignite.

The specific objectives of this research include a study on the transformation of pyrite, sulphate and organic sulphur during lignite pyrolysis as well as an investigation of the effect of inorganic matter on the sulphur transformation during lignite pyrolysis. To help the interpretation of sulphur transformation, the lignite samples were characterised using a combination of analytical techniques, i.e. X-ray diffraction (XRD), X-ray fluorescent (XRF), ion chromatography (IC), Scanning electron microscope equipped with an energy-dispersive X-ray spectroscopy (SEM-EDS), Fourier transform infra red (FTIR), solid state ${ }^{13} \mathrm{C}$ nuclear magnetic resonance (NMR) and petrographic analysis. Pyrolysis was carried out in a thermogravimetric analyser (TGA) and fixed bed reactor. The lignites and their chars were analysed for sulphur forms using a carbon sulphur (CS) analyser. To study the volatiles released during pyrolysis, experiments were also performed using a TGA coupled to a mass spectrometer (TGA-MS).

Eleven (11) Australian lignite samples, denoted as L1 to L11, respectively, from the same lignite deposit but with different sulphur and inorganic matter concentrations were employed in this study. They are categorised as of low quality since they contain high 
moisture, very high ash, low fixed carbon and thus they have low calorific value, except for L1. The lignites contain considerable amount of total sulphur, except for L1. Mineralogy of the lignites showed that the lignites contain extremely high sodium and chlorine. FTIR and solid state ${ }^{13} \mathrm{C}$ NMR spectroscopy confirmed that oxygenated functional structures are significantly present in the lignites.

Transformation of pyrite in the lignite during pyrolysis was studied using samples of a lignite with pyrite-free lignite (L1), a high pyrite lignite (L2), acid-washed lignites (AW L1 and AW L2), a pyrite mineral, and AW L1 blended with various amounts of the pyrite minerals. It was shown that in nitrogen the pyrite mineral $(\mathrm{S} / \mathrm{Fe}=2)$ decomposes to troilite $(\mathrm{S} / \mathrm{Fe}=1)$ at ca $1200 \mathrm{~K}$ and above this temperature, the troilite further decomposes to form elemental iron $(\mathrm{S} / \mathrm{Fe}<1)$. However, when blended with the demineralised lignite, the pyrite mineral can be completely decomposed to troilite at $873 \mathrm{~K}$ as confirmed by FTIR, SEM-EDS and XRD analyses on the resulting chars. Using TGA-MS, it was revealed that hydrogen sulphide and a small quantity of sulphur dioxide were released during the pyrolysis of the pyrite-lignite blends. However, only sulphur dioxide was detected during the pyrolysis of the lignite with high pyrite content. By assuming that the pyrolysis follows the first-order reactions, kinetic parameters of the pyrolysis of the lignite samples and the pyrite-lignite blends were obtained. The activation energy values of the lignite samples decreased with increasing temperature.

The transformation of sulphate during pyrolysis of the lignite was studied using pure sulphates $\left(\mathrm{CaSO}_{4}, \mathrm{FeSO}_{4}\right.$ and $\left.\mathrm{Fe}_{2}\left(\mathrm{SO}_{4}\right)_{3}\right), \mathrm{L} 1$ and L4, and acid washed L1 doped with sulphates $\left(\mathrm{CaSO}_{4}+\mathrm{L} 1\right.$ and $\left.\mathrm{FeSO}_{4}+\mathrm{L} 1\right)$, respectively. The TGA experiments showed that $\mathrm{CaSO}_{4}$ decomposes between $1400-1700 \mathrm{~K}$ in nitrogen and a $50 / 50 \mathrm{~N}_{2} / \mathrm{CO}_{2}$ mixture, while in air $\mathrm{CaSO}_{4}$ decomposes between 1500-1700 K. Using a TGA-MS it was found that only a small fraction of $\mathrm{CaSO}_{4}$ in $\mathrm{CaSO}_{4}+\mathrm{L} 1$ decomposed at $653 \mathrm{~K}$, releasing $\mathrm{SO}_{2}$. 
$\mathrm{FeSO}_{4} 7 \mathrm{H}_{2} \mathrm{O}$ released the bound water below $543 \mathrm{~K}$ and the remaining $\mathrm{FeSO}_{4}$ decomposed between 813 and $953 \mathrm{~K}$. $\mathrm{FeSO}_{4}$ in $\mathrm{FeSO}_{4}+\mathrm{L} 1$ decomposed at $500 \mathrm{~K}$ to release $\mathrm{SO}_{2}$. The inherent sulphates in $\mathrm{L} 4$ were dominated by iron sulphates which started to decompose and release $\mathrm{SO}_{2}$ at around $500 \mathrm{~K}$ and all sulphate had been decomposed at $1073 \mathrm{~K}$.

Transformation of organic sulphur in the lignite during pyrolysis was studied using samples of a lignite with high organic sulphur and mineral content (L3) and its acid washed lignite (AW L3), as well as naphthalene thiol and benzyl sulphoxide impregnated in a low sulphur lignite (AW L1) samples. It was shown that naphthalene thiol and benzyl sulphoxide in their impregnated lignites decomposes below $673 \mathrm{~K}$. It was observed that the retention of organic sulphur in the high organic sulphur lignite was higher than in its acid washed lignite. This is due to the interaction between inherent inorganic matter presents in the lignite with the organic sulphur retaining the organic sulphur in the solid phase. It also was revealed that sulphur dioxide is the only sulphur gas produced during pyrolysis of acid washed high organic sulphur lignite.

The effect of inherent and added inorganic matter on the transformation of organic and inorganic sulphur during pyrolysis of the lignite was studied by comparing the amounts of various forms of sulphur retained in the chars of a lignite contains high organic sulphur and inherent inorganic matter (L3), its acid washed lignite (AW L3), and the acid washed lignite doped with sodium and kaolinite. Inherent inorganic matter was shown to reduce the decomposition of inorganic and organic sulphur during lignite pyrolysis. Sodium helps the retention of sulphur in the chars between $400-500^{\circ} \mathrm{C}$ while kaolinite has a better ability to retain sulphur above $600^{\circ} \mathrm{C}$. 
Executive Summary

The present thesis has extended the fundamental knowledge of sulphur transformation during pyrolysis of lignite 


\section{LIST OF PUBLICATIONS}

\section{Journal Publications}

1. Setyawati Yani and Dongke Zhang, Transformation of Organic and Inorganic Sulphur in a Lignite during Pyrolysis : Influence of Inherent and Added Inorganic Matter, Proceedings of the Combustion Institute 32 (2009) 20832089

\section{Conference Publications}

1. Setyawati Yani and Dongke Zhang, An Experimental Study of the Decomposition of Pyrite in a Lignite During Pyrolysis, CHEMECA 2007, Melbourne, Australia, 23-26 September 2007

2. Setyawati Yani and Dongke Zhang, Organic Sulphur Transformation during Lignite Pyrolysis, $7^{\text {th }}$ Asia-Pacific Conference on Combustion, National Taiwan University, Taipei, Taiwan, 24-27 May 2009 


\section{TABLE OF CONTENTS}

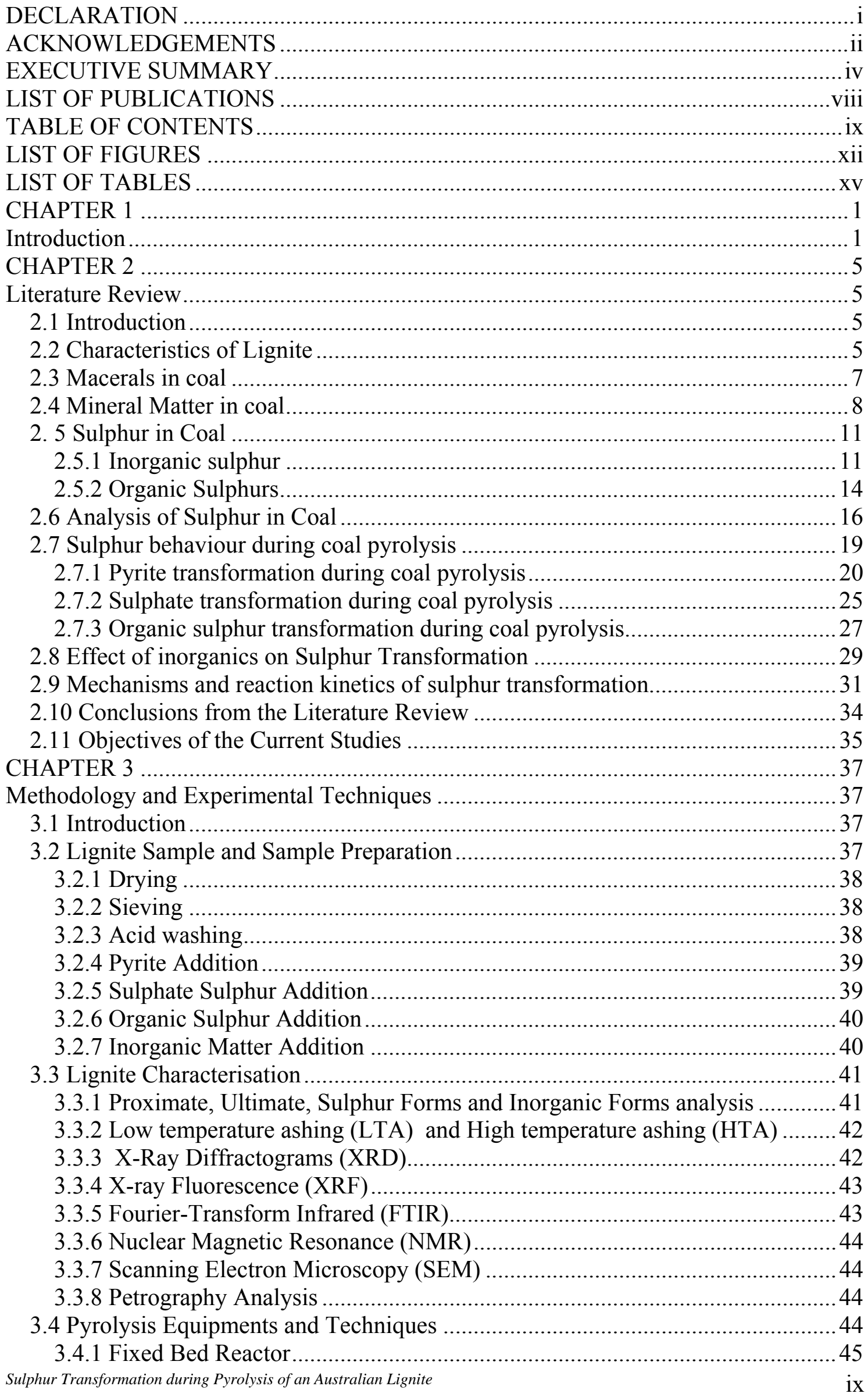




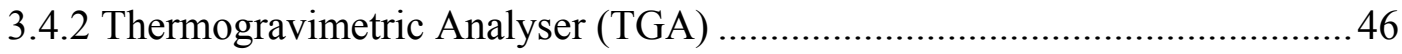

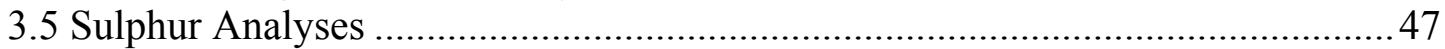

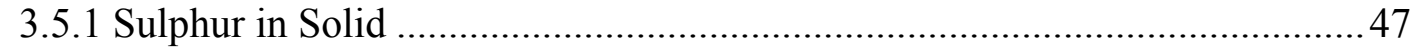

3.5.2 Sulphur in Gas Released during Pyrolysis ................................................. 49

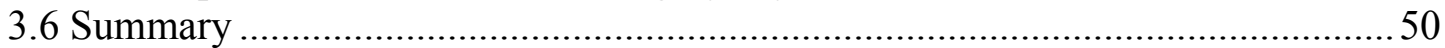

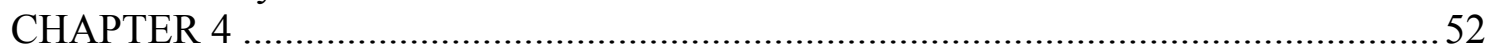

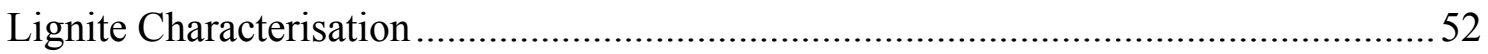

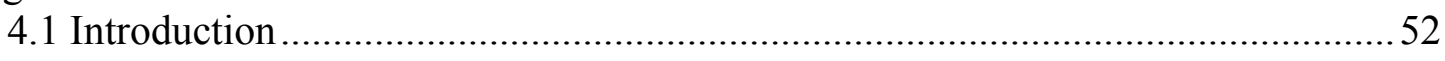

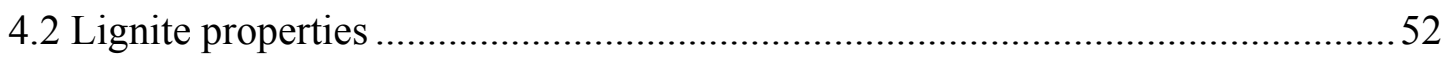

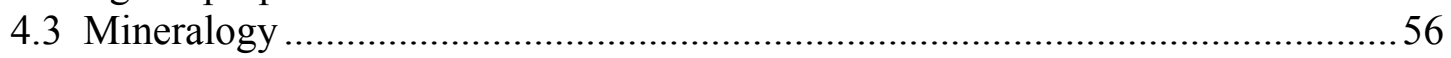

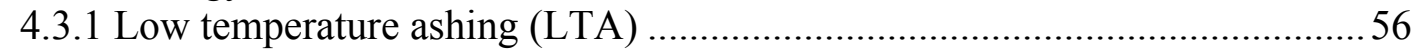

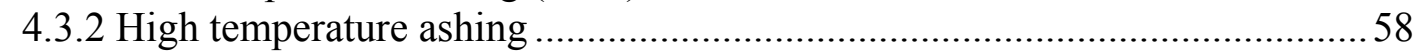

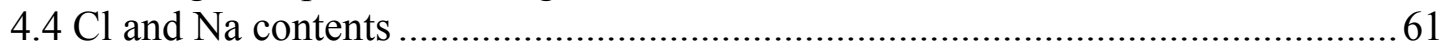

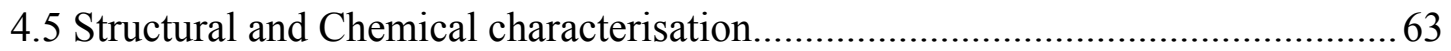

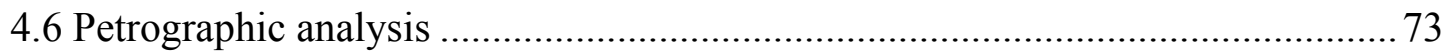

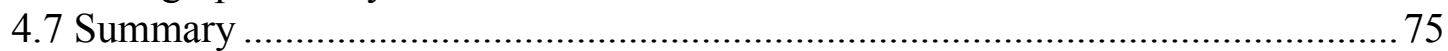

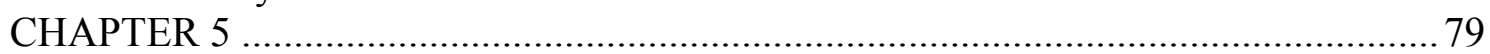

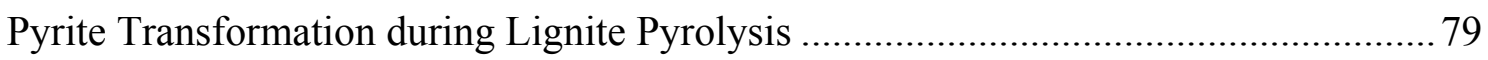

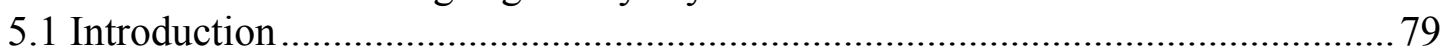

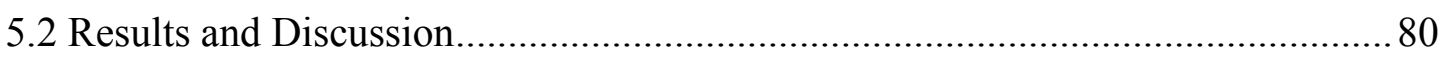

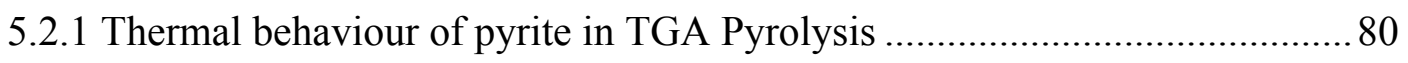

5.2.2 Thermal behaviour of lignite and lignite - pyrite blends............................. 82

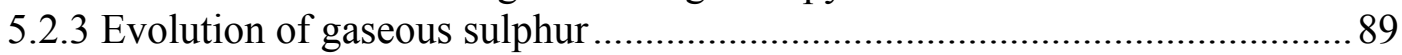

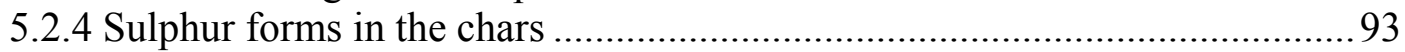

5.2.5 Kinetics of pyrolysis of pyrite, lignite, and lignite - pyrite blends ............... 101

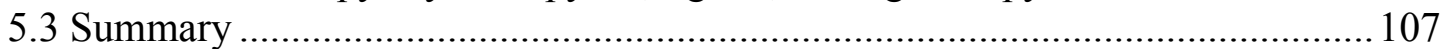

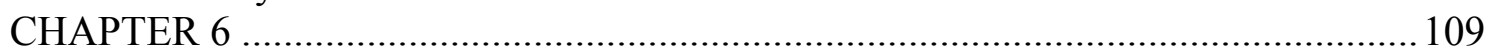

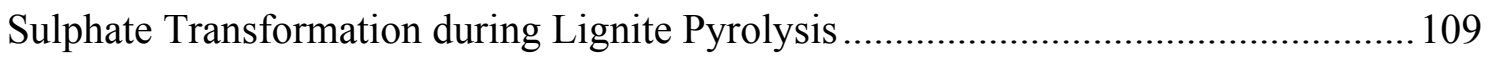

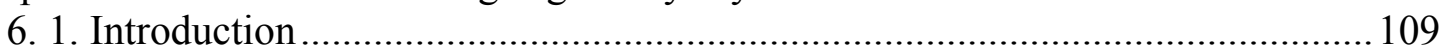

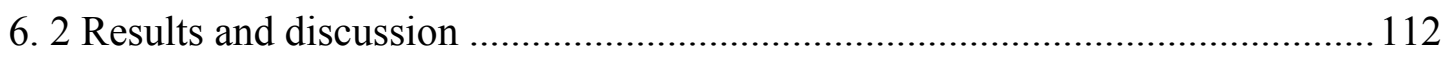

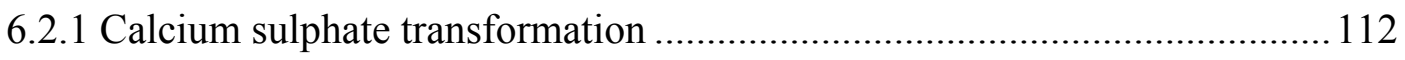

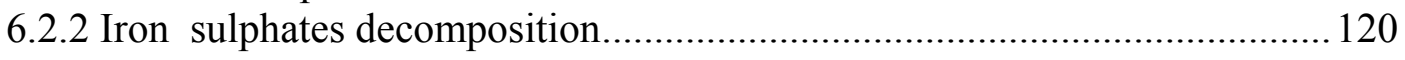

6.2.3 Transformation of inherent sulphate during lignite pyrolysis.................... 127

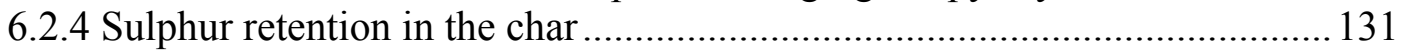

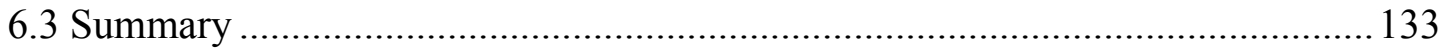

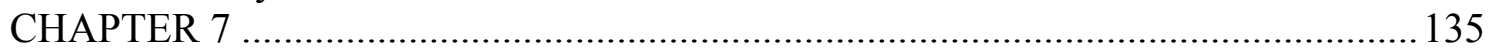

Organic Sulphur Transformation during Lignite Pyrolysis ..................................... 135

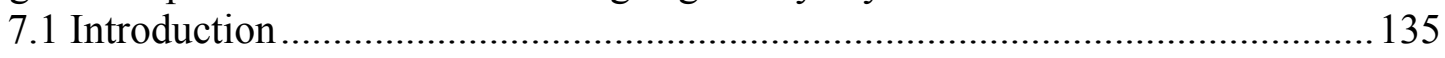

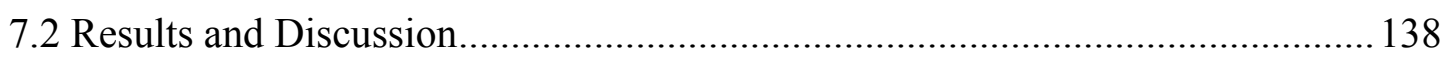

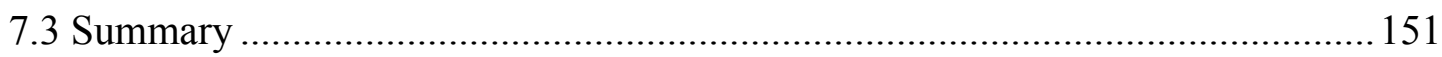

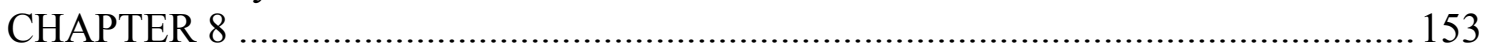

The effect of Inorganic Matter on Sulphur Transformation during Lignite Pyrolysis.. 153

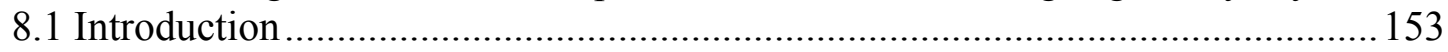

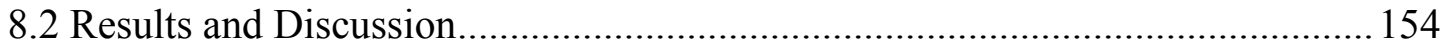

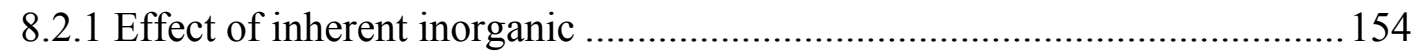

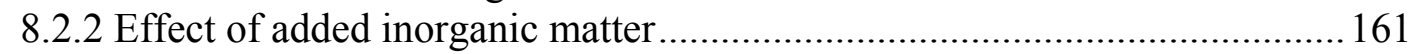

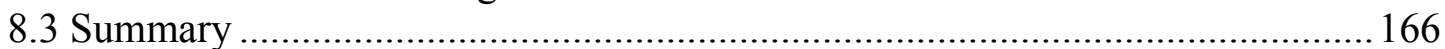

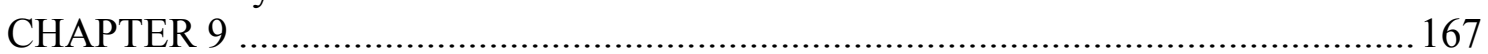

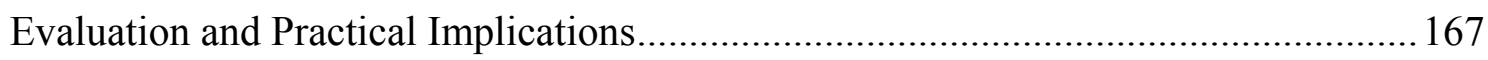

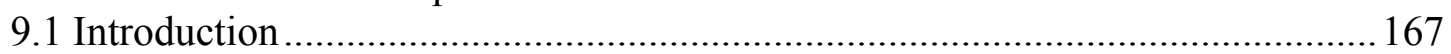

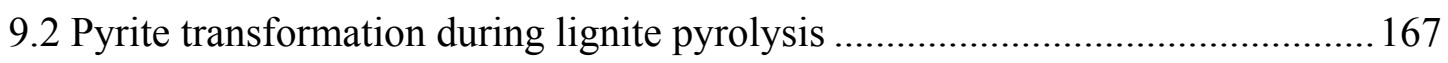

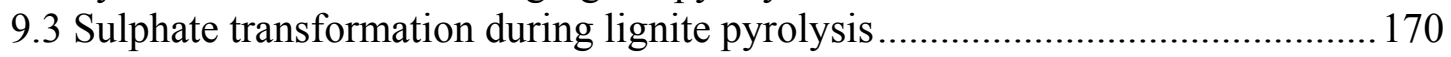

$\begin{array}{lc}\text { Sulphur Transformation during Pyrolysis of an Australian Lignite } & \mathrm{X}\end{array}$ 
9.4 Organic sulphur transformation during lignite pyrolysis 172

9.5 A proposed mechanisms of sulphur transformation during lignite pyrolysis .... 175

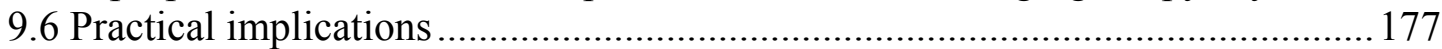

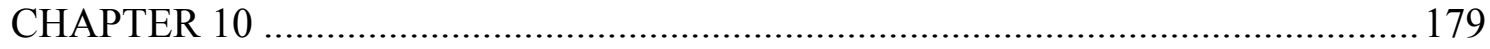

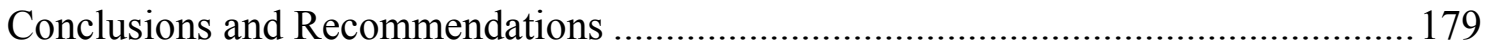

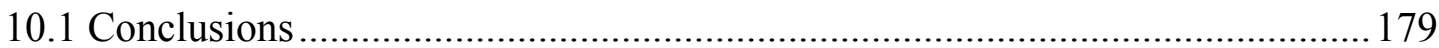

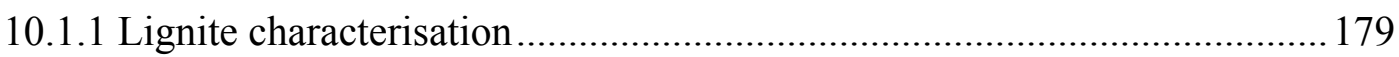

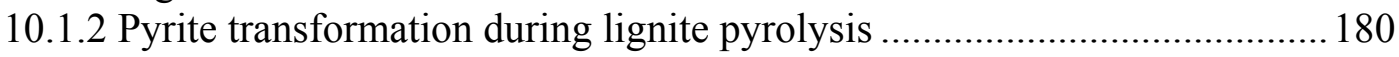

10.1.3 Sulphate transformation during lignite pyrolysis .................................. 181

10.1.4. Organic sulphur transformation during lignite pyrolysis ........................ 182

10.1.5 Effect of inorganic matter on the sulphur transformation during lignite

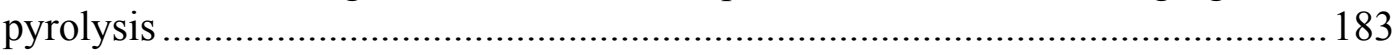

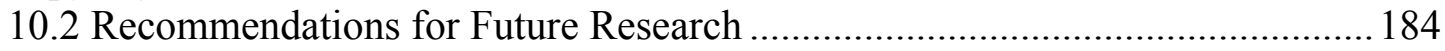

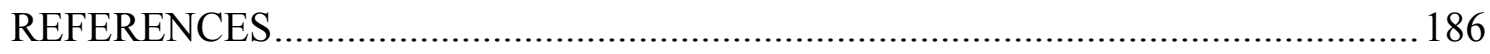

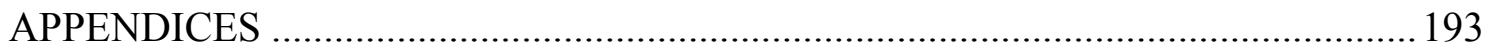

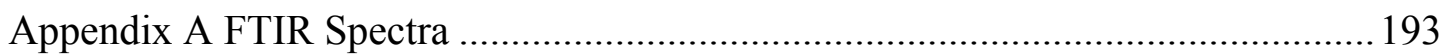

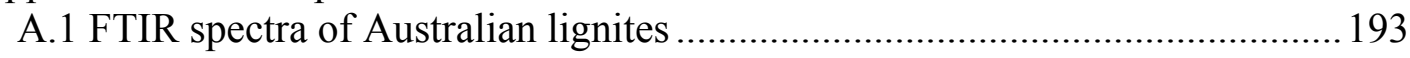

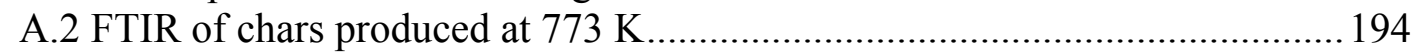

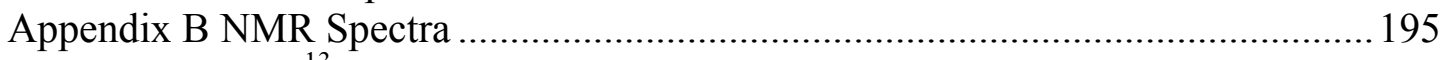

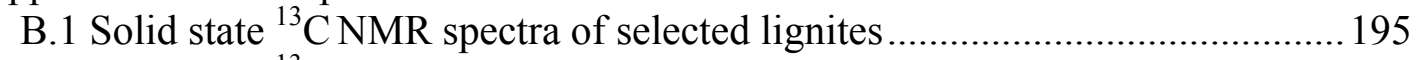

B.2 Solid state ${ }^{13} \mathrm{C}$ NMR spectra of selected chars at $773 \mathrm{~K}$................................. 196

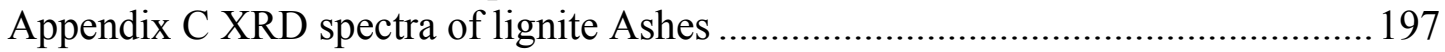

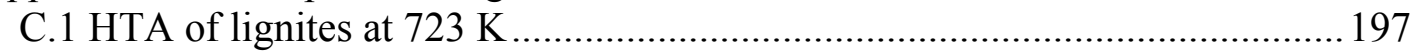

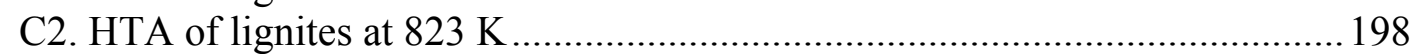

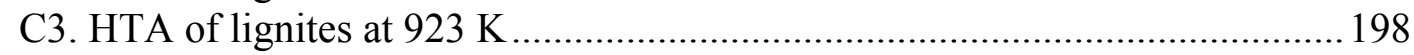




\section{LIST OF FIGURES}

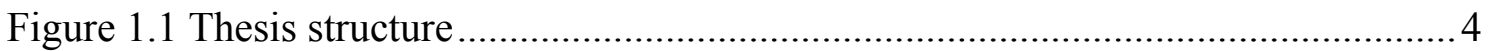

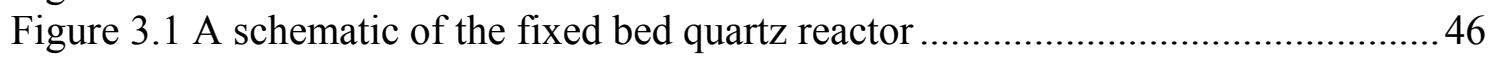

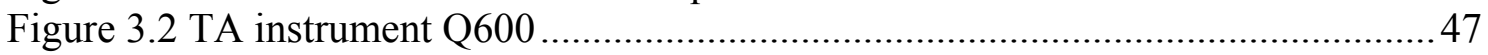

Figure 3.3 Eltra CS 2000 ................................................................................. 48

Figure 3.4 Determination of various forms of sulphur in lignites and chars .................. 48

Figure 3.5 Netzch PC409 TGA-DSC coupled to a 403C Aeolos Netzch mass

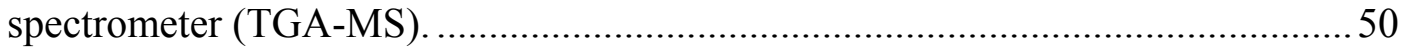

Figure 4.1. X-ray diffractograms of LTA samples L2, L3, L5, L7 .............................56

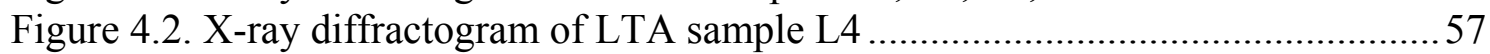

Figure 4.3. X-ray diffractogram of LTA sample L11 …................................................. 58

Figure 4.4. X-ray diffractogram of sample L2 ash prepared at three different

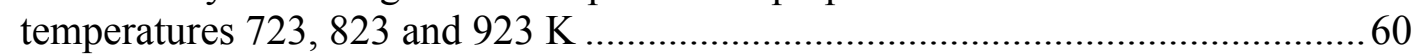

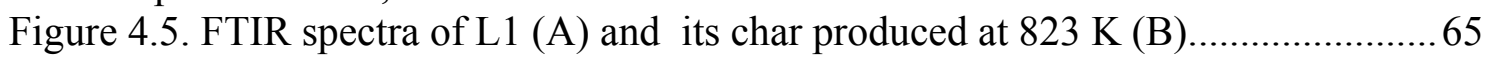

Figure 4.6. FTIR spectra of L2 (A) and its char produced at $823 \mathrm{~K}(\mathrm{~B}) \ldots \ldots \ldots \ldots \ldots \ldots \ldots . . . . . . . . .66$

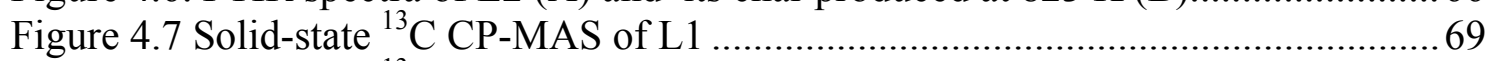

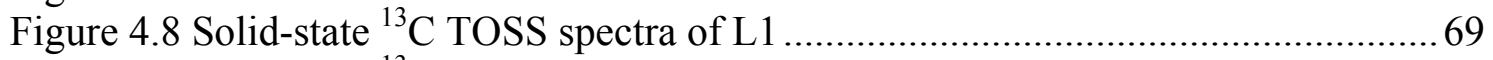

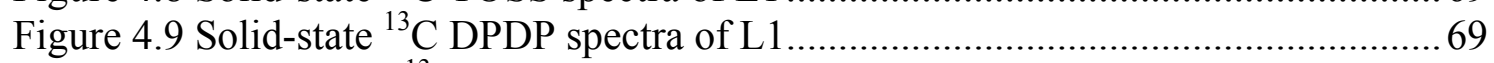

Figure 4.10 Solid-state ${ }^{13} \mathrm{C}$ CP-MAS spectra of Western Australian lignite char ............. 71

Figure 4.11 Solid-state ${ }^{13} \mathrm{C}$ TOSS spectra of Western Australian lignite char ................... 71

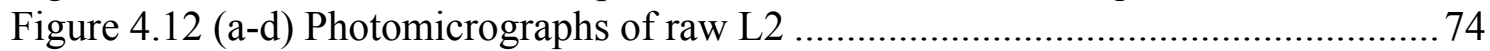

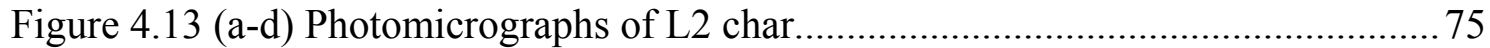

Figure 5.1. TGA mass loss and Fe/S ratio profiles of pyrite decomposition ............ 81

Figure 5.2. TGA mass loss profiles and DTG thermograms on dry basis of L1, AW

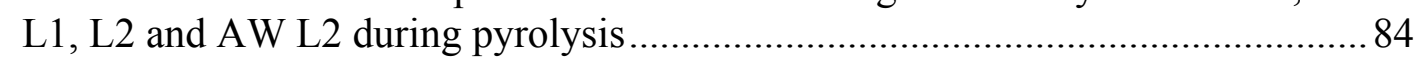

Fig 5.3. TGA mass loss profiles on dry ash free (daf) basis of L1, AW L1, L2 and AW

L2 during pyrolysis ..................................................................................... 85

Figure 5.4. TGA mass loss profiles and DTG thermograms on dry basis during pyrolysis of pyrite, AW L1 and lignite-pyrite blends .......................................... 86

Figure 5.5. Calculated mass loss profiles (dry basis) during the pyrolysis of pyrite,

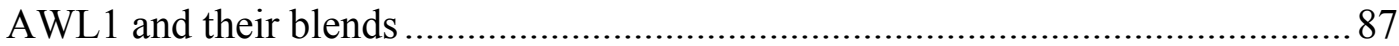

Figure 5.6. Evolution of $\mathrm{H} 2 \mathrm{~S}(\mathrm{~m} / \mathrm{z} 33$ and 34) during pyrolysis of the 10P-90L 1 in TGA-MS 89

Figure 5.7. Evolution of $\mathrm{SO} 2(\mathrm{~m} / \mathrm{z} 48$ and 64) during pyrolysis of L2, AW L2, AW

L1 and 10P-90L1 in TGA-MS ..................................................................... 90

Figure 5.8. FTIR spectra of L2, AW L2, L1, AW L1, and various pyrite-lignite blends

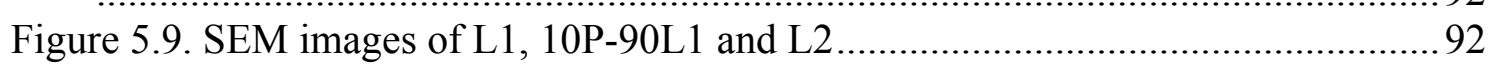

Figure 5.10. Total sulphur retention in chars produced at 573-1073 K during fixed bed

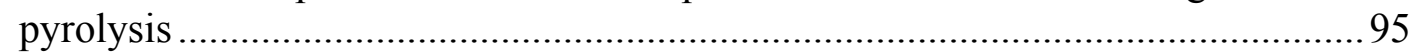

Figure 5.11. Inorganic sulphur retention of chars produced at 573-1073 K during fixed

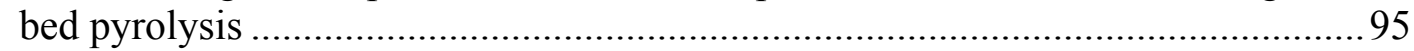

Figure 5.12. FTIR spectra of 10P-90L1 and its chars at different pyrolysis

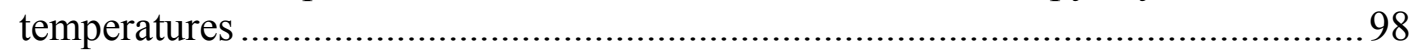

Figure 5.13. SEM images of the 10P-90L1 sample before pyrolysis (A) and its char produced at $673 \mathrm{~K}(\mathrm{~B}), 873 \mathrm{~K}(\mathrm{~A})$, and $1073 \mathrm{~K}(\mathrm{D})$, respectively............................ 99

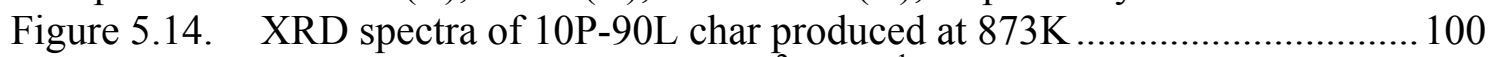

Figure 5.15. A typical plot of $\ln \left(-\ln (1-\mathrm{X}) \cdot \mathrm{T}^{-2}\right)$ vs $\mathrm{T}^{-1}$ for lignite pyrolysis ................... 103

Figure 6.1 TGA and DTG profiles of $\mathrm{CaSO}_{4}$ decomposition in $\mathrm{N}_{2}, \mathrm{~N}_{2} / \mathrm{CO}_{2}$ mixture and

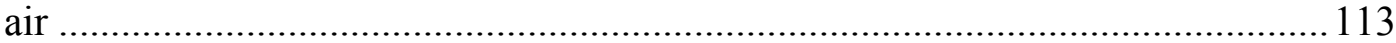

Sulphur Transformation during Pyrolysis of an Australian Lignite xii 
Figure 6.2 TGA and DTG profiles of thermal decomposition of $\mathrm{CaSO}_{4}+\mathrm{L} 1$ in a TGA in nitrogen atmosphere

Figure $6.3 \mathrm{SO}_{2}$ evolution (m/z 64 and $\left.\mathrm{m} / \mathrm{z} 48\right)$ during pyrolysis of $\mathrm{AW} \mathrm{L1}$ and $\mathrm{CaSO}_{4}+\mathrm{L} 1$ in a TGA-MS

Figure 6.4 $\mathrm{SO}_{2}$ evolution during pyrolysis of $\mathrm{AW} \mathrm{L1}$ and $\mathrm{CaSO}_{4}+\mathrm{L} 1$ and from the decomposition of $\mathrm{CaSO}_{4}$

Figure 6.5 SEM backscattered images of $\mathrm{CaSO}_{4}+\mathrm{L} 1$ low magnification and high magnification (a-b) and its EDS spectra ( c ).

Figure 6.6 SEM backscattered images $\mathrm{CaSO}_{4}+\mathrm{L} 1$ chars produced at $1073 \mathrm{~K}$ low and high magnification (a-b) and its EDS spectra ( c ).....

Figure 6.7 XRD patterns of the char of $\mathrm{CaSO}_{4}+\mathrm{L} 1$ produced at $1073 \mathrm{~K}$

Figure 6.8 TGA and DTG profiles of $\mathrm{Fe}_{2}\left(\mathrm{SO}_{4}\right)_{3}$ decomposition in $\mathrm{N}_{2}, \mathrm{~N}_{2} / \mathrm{CO}_{2}$ mixture and air.

Figure 6.9 TGA and DTG profiles of $\mathrm{FeSO}_{4}$ decomposition in $\mathrm{N}_{2}, \mathrm{~N}_{2} / \mathrm{CO}_{2}$ mixture and air

Figure 6.10 TGA and DTG profiles of the pyrolysis of $\mathrm{FeSO}_{4}+\mathrm{L} 1$ in nitrogen atmosphere.

Figure 6.11 Comparison of the thermal decomposition of pure $\mathrm{CaSO}_{4}$ (solid line (-))

and FeSO4 (broken line (---)) in a TGA under nitrogen......

Figure 6.12 Comparison of the thermal decomposition of $\mathrm{CaSO}_{4}+\mathrm{L} 1$ and $\mathrm{FeSO}_{4}+\mathrm{L} 1$ in a TGA under nitrogen atmosphere.

Figure $6.13 \mathrm{SO}_{2}$ evolution during pyrolysis of $\mathrm{FeSO}_{4}+\mathrm{L} 1$ and $\mathrm{AW} \mathrm{L} 1$

Figure $6.14 \mathrm{SO}_{2}$ evolution, sum of $\mathrm{m} / \mathrm{z} 64+\mathrm{m} / \mathrm{z} 48$, during pyrolysis of $\mathrm{AW} \mathrm{L} 1$ and $\mathrm{FeSO}_{4}+\mathrm{L} 1$ and from the decomposition of $\mathrm{FeSO}_{4} \ldots$

Figure 6.15 SEM backscattered images of $\mathrm{FeSO}_{4}+\mathrm{L} 1$ low and high magnification (a-b) and its EDS spectra ( c ).

Figure 6.16 SEM backscattered images of $\mathrm{FeSO}_{4}+\mathrm{L} 1$ char produced at $1073 \mathrm{~K}$ low and

high magnification (a-b) and its EDS spectra ( c )...

Figure 6.17 FTIR spectra of L4 and its chars at $673 \mathrm{~K}$ and $1073 \mathrm{~K}$

Figure $6.18 \mathrm{SO}_{2}$ evolution during pyrolysis of L4 in a TGA under argon atmosphere 129

Figure 6.19 Comparison of retention of the total sulphur in chars from $\mathrm{FeSO}_{4}+\mathrm{L} 1$,

$\mathrm{CaSO}_{4}+\mathrm{L} 1$ and $\mathrm{L} 4$ during pyrolysis in the fixed-bed reactor

Figure 6.20 Comparison between volatile matter release and volatile sulphur release during pyrolysis of the $\mathrm{FeSO}_{4}+\mathrm{L} 1, \mathrm{CaSO}_{4}+\mathrm{L} 1$ and $\mathrm{L} 4$ during pyrolysis in the fixed-bed reactor

Figure 7.1 Comparison of retention of the total sulphur in chars from L3, AW L3,

$\mathrm{NT}+\mathrm{L} 1, \mathrm{BS}+\mathrm{L} 1$ during pyrolysis in the fixed-bed reactor.

Figure 7.2 Comparison of retention of the organic sulphur in chars from L3, AW L3,

$\mathrm{NT}+\mathrm{L} 1, \mathrm{BS}+\mathrm{L} 1$ during pyrolysis in the fixed-bed reactor.....

Figure 7.3 FTIR spectra of (a) AW L1, (b) naphthalene thiol, (c) NT+L1, (d) NT+L1 char at $673 \mathrm{~K}$ and (e) NT+L1 char at $1073 \mathrm{~K}$

Figure 7.4 SEM images of (a) NT $+\mathrm{L} 1$, (b) $\mathrm{NT}+\mathrm{L} 1$ char at $673 \mathrm{~K}$ and (c) $\mathrm{NT}+\mathrm{L} 1$ char at $1073 \mathrm{~K}$

Figure 7.5 SEM images of (a) BS+L1, (c) BS $+\mathrm{L} 1$ char at $673 \mathrm{~K}$,(d) $\mathrm{BS}+\mathrm{L} 1$ char at

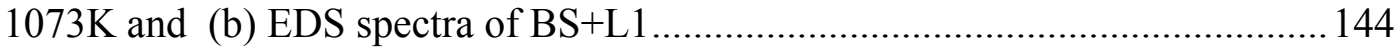

Figure 7.6 SEM images and EDS spectra of AW L3 ( $a$ and $b$ ) and L3 (c and d) ......... 145

Figure 7.7 FTIR spectra of (a) L3, (b) AW L3, (c) AW L3 char at $673 \mathrm{~K}$ and (d) AW

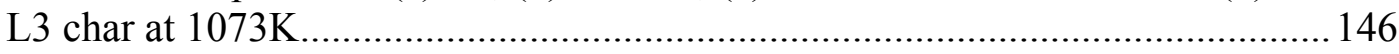

Figure 7.8 Evolution of $\mathrm{SO}_{2}(\mathrm{~m} / \mathrm{z} 64$ and $\mathrm{m} / \mathrm{z} 48)$ during pyrolysis of AW L3 in a TGAMS.

Figure 7.9 Evolution of $\mathrm{CO}_{2}(\mathrm{~m} / \mathrm{z} 44)$ during pyrolysis of AW L3 in a TGA-MS ....... 149 
Figure 7.10 Evolution of $\mathrm{H}_{2} \mathrm{O}(\mathrm{m} / \mathrm{z} 17$ and $\mathrm{m} / \mathrm{z} 18)$ during pyrolysis of $\mathrm{AW} \mathrm{L} 3$ in a TGA-MS

Figure 8.1 The retention of total sulphur, organic sulphur and inorganic sulphur in the

L 2 chars during fixed bed pyrolysis....

Figure 8.2 The retention of total sulphur, organic sulphur and inorganic sulphur in

AWL2 chars during fixed bed pyrolysis

Figure 8.3 A comparison of organic sulphur in L2 and AW L2 chars during fixed bed pyrolysis

Figure 8.4 A comparison of inorganic sulphur retention in L2 and AW L2 chars during

fixed bed pyrolysis

Figure 8.5 The retention of total sulphur, organic sulphur and inorganic sulphur in L3 chars during fixed bed pyrolysis

Figure 8.6 The retention of total sulphur, organic sulphur and inorganic sulphur in AWL3 chars during fixed bed pyrolysis

Figure 8.7 A comparison of organic sulphur retention in L3 and AW L3 chars during fixed bed pyrolysis

Figure 8.8 A comparison of inorganic sulphur retention in L3 and AW L3 chars during fixed bed pyrolysis

Figure 8.9 SEM images and EDS spectra of AW L3 (a-b), AW+Na (c-d) and AW+kaolin (e-f)

Figure 8.10 A comparison of total sulphur retention in L3, AW L3, AW+Na,

AW+kaolin chars during fixed bed pyrolysis

Figure 8.11 A comparison of organic sulphur retention in L3, AW L3, AW+Na, $\mathrm{AW}+$ kaolin chars during fixed bed pyrolysis

Figure 8.12 A comparison between volatile matter release and volatile sulphur release during pyrolysis of $\mathrm{L} 3, \mathrm{AW} \mathrm{L} 3, \mathrm{AW}+\mathrm{Na}$ and $\mathrm{AW}+$ kaolin during pyrolysis in the fixed-bed reactor

Figure 9.1 Comparison of retentions of organic sulphur during pyrolysis of L3 and AW L3 in a fixed bed reactor with pyrolysis of Datong (DT) and Yima (YM) in a fluidized bed reactor by Qi et al [127]

Figure 9.2 A schematic of the mechanisms of sulphur transformation during lignite pyrolysis

Figure A.1 FTIR spectra of L3 to L11, respectively

Figure A.2 FTIR spectra of L3-L11 chars produced at $773 \mathrm{~K}$

Figure B.1 Solid state ${ }^{13}$ C NMR spectra of L1, L3, L4, L9, L10 and L11, respectively

Figure B.2 Solid state ${ }^{13} \mathrm{C}$ NMR spectra of lignite chars produced at $773 \mathrm{~K} \ldots \ldots \ldots \ldots \ldots . . . .196$

Figure C.1 X-ray diffractogram of lignite ash prepared at 723 K............................... 197

Figure C.2 X-ray diffractogram of lignite ash prepared at 823 K............................... 198 


\section{LIST OF TABLES}

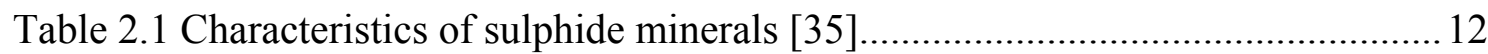

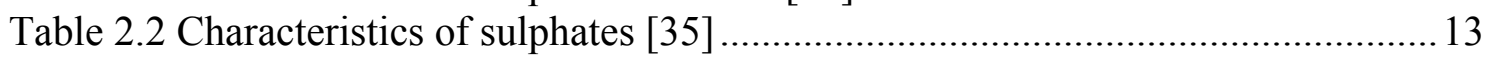

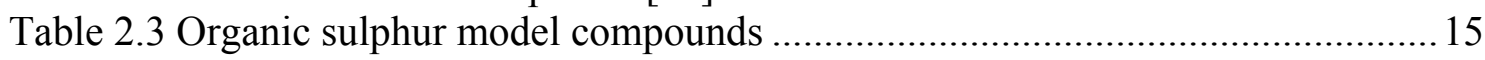

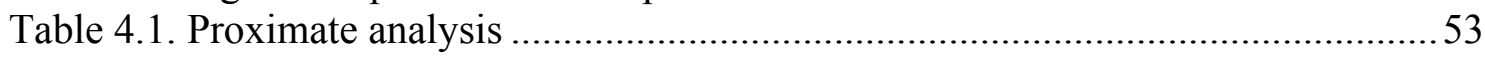

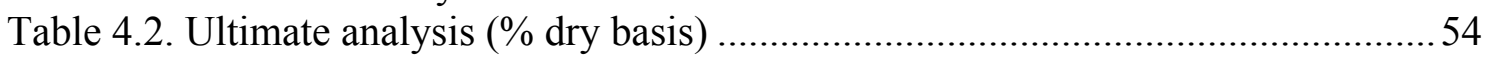

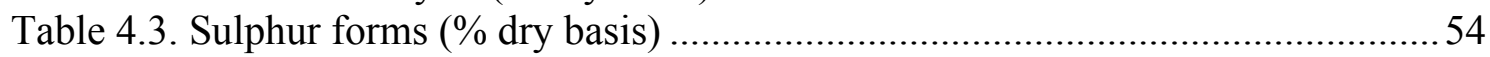

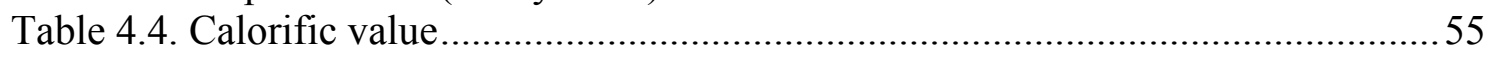

Table 4.5. Mineralogy of LTA samples (A2, B2, C1 and D) using powder XRD and

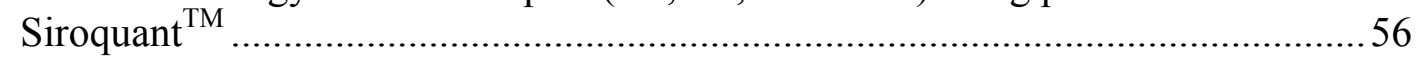

Table 4.6. Ash yield of samples prepared at different temperatures............................59

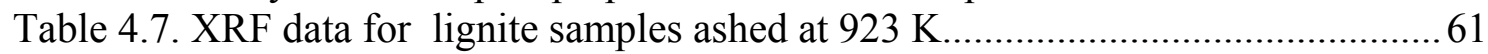

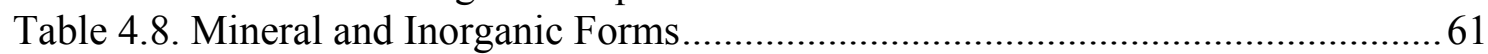

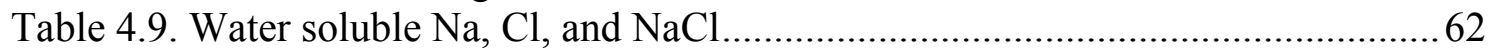

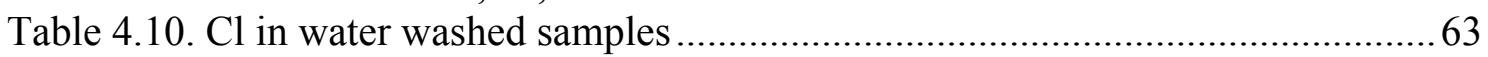

Table 4.11. Band assignments of the most prominent peaks in the FTIR spectra ..........65

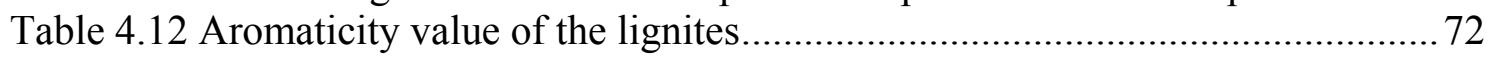

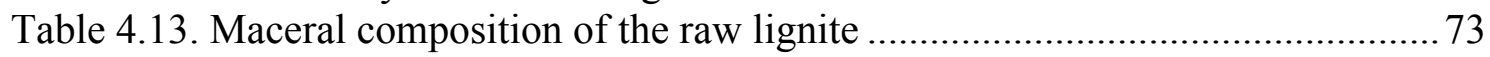

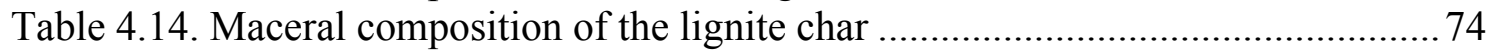

Table 5.1 Total, organic and inorganic sulphur contents of the lignite samples............ 83

Table 5.2. Kinetic parameters of pyrolysis of lignites, pyrite and lignite-pyrite blends

Table 6.1 Comparison of decomposition temperatures of selected sulphates [9, 38, 60, $110,112-114]$

Table 7.1 A summary of possible thermal decomposition reactions of organic sulphur

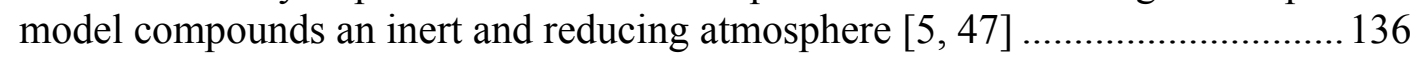

Table 7.2 Structure and physical properties of sulphur model compounds .................. 138

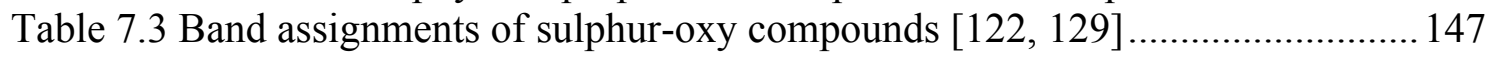

Table 9.1 Comparison of experimental techniques and conditions for thermal decomposition of pyrite between the current study and that of Chen et al [7] .... 168

Table 9.2 A comparison of sulphate decomposition in the current study and that of Yan et al [9].

Table 9.3 A comparison of sulphur forms analysis and experimental data of current study and Qi et al [127] study 


\section{CHAPTER 1}

\section{Introduction}

Lignite as a primary energy source will become increasingly important in Australia's total energy mix in the future in the face of rapidly rising oil price and because of its abundance, easy access and low cost of mining. Australia has about $24 \%$ of the global economical lignite resources, ranking the first in the world [1]. However, the utilisation of lignite is faced with a number of technical, economical and environmental challenges due to its high moisture content (and thus low specific energy) and some of the deposits containing high sulphur and high inorganic matter [2, 3]. Lignites with high sulphur and inorganic matter cannot be utilised in conventional high-temperature combustion or gasification technologies without significant economic and environmental penalties. Low-temperature pyrolysis to yield liquid fuels from condensed volatile matter and upgrading of the resultant char as value-added carbon products present a potentially low-cost alternative. Besides, pyrolysis is the first step of any thermochemical conversion processes of coal. The present contribution of this thesis examines the transformation of sulphur during pyrolysis of a Western Australian lignite.

Generally speaking, all coals contain some sulphur in two major forms, viz, inorganic and organic sulphur. The inorganic sulphur exists as pyrite $\left(\mathrm{FeS}_{2}\right)$ and sulphates (mainly as iron and calcium sulphates). Sulphate sulphur is usually not present in significant quantities in fresh coals but can be formed in partially weathered coals (oxidation of pyrite). Organic sulphur is primarily present as thiolic, sulphidic, and thiophenic structures in the organic matrix of a coal. The amount of thiol and disulphide sulphurs is usually high in lignite, whereas thiophene is present substantially in high-rank coals. The higher the coal rank, the lower the labile sulphur contents $[4,5]$. 
Chapter 1

During pyrolysis, pyrite $\left(\mathrm{FeS}_{2}\right)$ contained in a coal is transformed into a more reactive ferrous sulphide at temperatures above $360^{\circ} \mathrm{C}[6,7]$. This active sulphur then reacts with hydrogen in the coal to form hydrogen sulphide $\left(\mathrm{H}_{2} \mathrm{~S}\right)$ or reacts with active sites of organic matrix in the char structure to form new organic sulphur $[7,8]$. Decomposition of sulphates in coal during pyrolysis occurs at temperatures between 200 and $500^{\circ} \mathrm{C}$ and depends on the type of sulphates: iron sulphate decomposes at a lower temperature than calcium sulphate [9]. Sulphur dioxide $\left(\mathrm{SO}_{2}\right)$ is evolved during decomposition of sulphates in coal [9]. Organic sulphur decomposes according to its stability. The more labile the organic sulphur, the lower the decomposition temperature. In an inert atmosphere, thiols decompose between $200-300^{\circ} \mathrm{C}$, aliphatic sulphide and disulphide between $350-500^{\circ} \mathrm{C}$, cyclic sulphide and aryl sulphide between $700-800^{\circ} \mathrm{C}$, and thiophenes above $900^{\circ} \mathrm{C}[4,5,9]$.

Once the sulphur compounds evolve they combine with moisture in the atmosphere to form acid rain which has been known to have detrimental effect to the environment as well as human health and a source of acid rain. These problems lead to stringent legislation and control of $\mathrm{SO}_{\mathrm{x}}$ emission from power generation plants.

The retention of both organic and inorganic sulphur during coal pyrolysis is influenced by inorganic matter present in the coal. Alkali and alkali earth metals react with sulphur to form low melting-point eutectics which cause slagging, fouling, agglomeration and defluidisation during coal utilisation [10]. Therefore, it is necessary to study the effect of inorganic matter on the sulphur transformations.

The overall aim of this research is to improve our understanding of the behaviour of sulphurs during pyrolysis of Western Australian lignite. This knowledge can lead to an alternative technology of utilising lignites with high sulphur and high inorganic matters. 
Chapter 1

This thesis is written in ten chapters which are interlinked with each other as portrayed in Figure 1.1.

The present chapter (Chapter 1) establishes the scope overall aims of this thesis and the need for further research into the sulphur transformation during pyrolysis of Western Australian lignite.

Chapter 2 extensively reviews previous investigations on this subject, including lignitesulphur chemistry and inorganic matter-sulphur interaction during lignite pyrolysis. It also identifies the gaps in the literature, which form the objectives of the present studies.

Chapter 3 describes all the experimental equipment and techniques employed in the present studies and the data analysis method.

The results, their analysis and their interpretation are presented in five chapters. The characterisation of the lignite is discussed in length in Chapter 4.

Chapters $5-7$ present the results of the finding of various types of sulphur behaviour during lignite pyrolysis. Chapter 5 discusses the pyrite transformation during lignite pyrolysis. Chapter 6 details the sulphate sulphurs transformation during lignite pyrolysis. Chapter 7 summaries the organic sulphur transformation during lignite pyrolsysis.

The interaction between inorganic matter and sulphurs during lignite pyrolysis is discussed in Chapter 8. Chapter 9 evaluates the results obtained in the present study, the practical implication and significance of the current results are also discussed. Chapter 10 provides a concise account of the conclusions over the whole study and outlines recommendations for future research and development. 


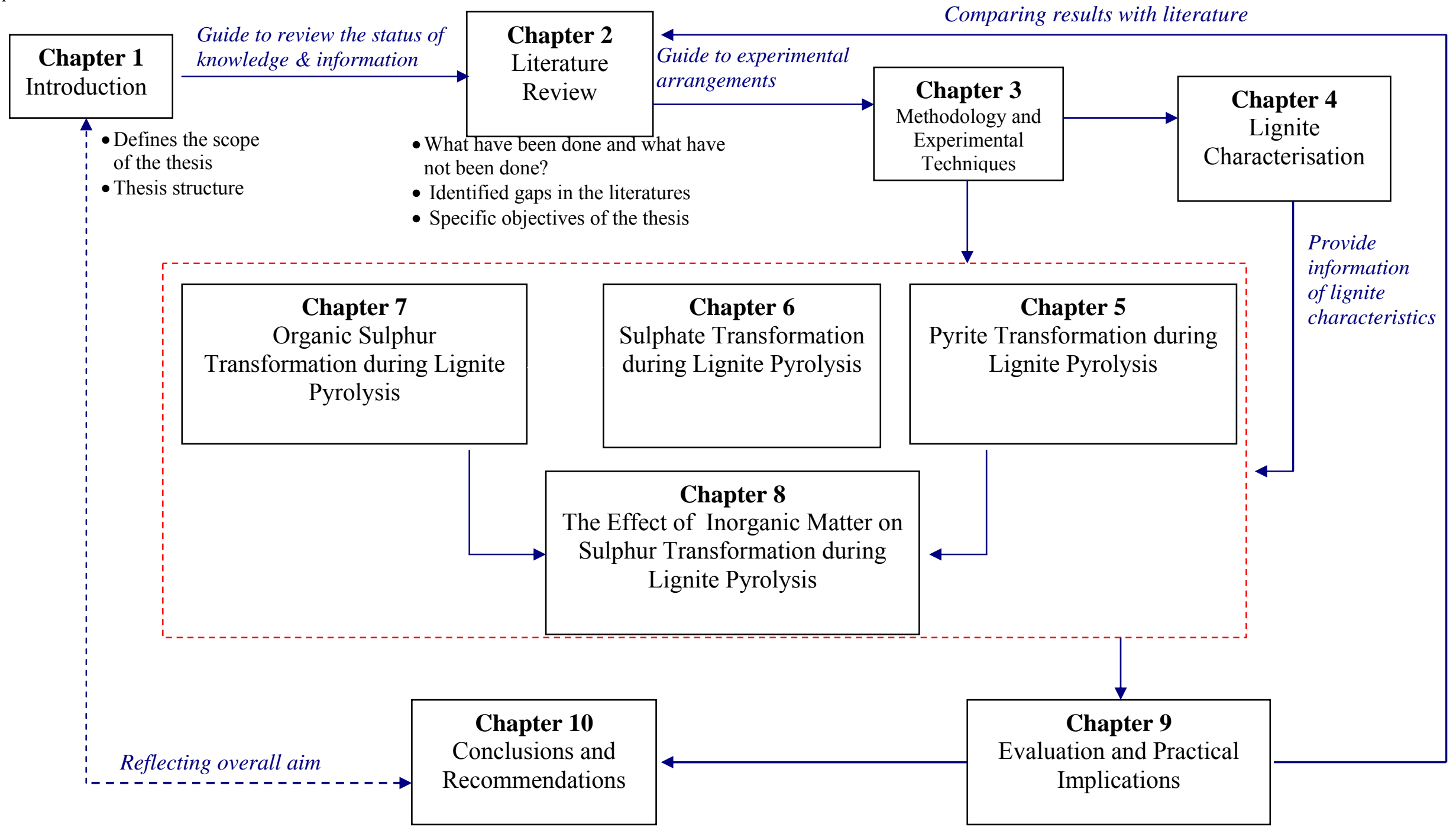

Figure 1.1 Thesis structure 


\section{CHAPTER 2}

\section{Literature Review}

\subsection{Introduction}

The presence of sulphur in lignite, although in small quantities, can dramatically affect lignite utilisation processes especially in terms of pollution control. Extensive research efforts have been conducted in order to understand the transformation of sulphur during lignite pyrolysis [2-6]. It is necessary to establish a solid knowledge base for the current studies by examining the information available on this subject.

This chapter reviews previous literature related to the transformation of sulphur during coal pyrolysis. The characteristics of lignite are reviewed to understand its effects to the behaviour of sulphur. The origin of sulphur in coal is also reviewed. Particular attention is paid to the transformation of various sulphur forms during coal pyrolysis in order to understand possible mechanisms of sulphur release. Attention is also paid to the effect of inorganic matters on sulphur transformation during coal pyrolysis. Through a critical evaluation of the previous work, the gaps in the literature are identified and the objectives of the current studies are established.

\subsection{Characteristics of Lignite}

Coal is a heterogeneous material which is composed from organic and inorganic materials. The organics are derived from plants which have undergone chemical and physical decomposition during burial. While mineral matters are derived from many sources, such as mineral transported by water or air, direct precipitation from solution, 
Chapter 2

precipitation by organic metabolism and secondary precipitation from ground water [11].

Plant remains and other sediments are accumulated in swamps and transformed to peat. Subsequently, peat was transformed, in the general time-sequence, to lignite, subbituminous, bituminous, and anthracite [12]. This transformation is called coalification and the degree to which the coalification has taken place is termed as coal rank. Thus lignite is classified as the youngest coal. Elemental analyses, volatile matter, specific energy determinations or petrographic analyses are employed to define coal rank. However, no one rank indicator is suitable for all ranks [13].

There is no single agreement of the definition of lignite. In some countries lignite is also known as brown coal. Australian coals are only classified into two rank classes according to classification and coding system for Australian coals [14], they are classified as higher rank coal if the gross calorific value $\geq 21 \mathrm{MJ} / \mathrm{kg}$ and as lower rank coal if the gross calorific value $<21 \mathrm{MJ} / \mathrm{kg}$. The American Society for Testing Materials (ASTM) ranks the coals, from the lowest to the highest, as follows: lignite, subbituminous, bituminous and anthracite. A coal is classified as lignite if the gross caloric value $<19.5 \mathrm{MJ} / \mathrm{kg}[15]$. The Alpern coal classification classifies carboniferous rocks into three groups; they are lignite, bituminous and anthracite. According to this classification, a coal which has reflectance ratio $(\mathrm{Rr})<0.6$ and calorific value $<24 \mathrm{MJ} / \mathrm{kg}$ is classified as lignite [16]. Another researcher [13] summaries the classification of coal according to ASTM coal rank. Based on this, lignite is an immature coal which was formed during diagenesis, it also has vitrinite reflectance $<0.3$, moisture content (in situ) $>25 \%$, carbon ca. $71 \%$, volatile matter ca. 49 and the microscopic characterisatics show marked gelification and compaction. The IEA coal research report [12] stated that lignites generally have moisture content between $35-70 \%$, low heat value (6-16 MJ/kg), 
Chapter 2

oxygen content above $20 \%$ on a dry-ash-free basis, lower fixed carbon and higher volatile matter than bituminous coals.

As immature coal, lignite has a great abundance of oxygenated functional groups which is revealed from the study of lignite structure using Fourier transform infrared (FTIR) spectroscopy and solid state 13C nuclear magnetic (NMR) spectroscopy [17-19]. Lignite contains less complex aliphatic structure than higher rank coals, the carbon aromaticity in lignite is also lower than in more mature coals [17].

\subsection{Macerals in coal}

Organic materials in coal are called macerals. The benefit of coal to the society, for example, as a source of energy and hydrocarbon components and also its role in metallurgical processing and in-situ methane captured, is derived from coal macerals [20]. The quantification of macerals in coal is carried out using a polished block of coal which is examined using a reflected light microscope [21]. There are three maceral groups present in hard black coals, they are vitrinite, liptinite, and inertinite [22]. In lignites (soft/brown coals), liptinite and inertinite have the same name as those in black coals, however vitrinite does not occur since the random reflectance $(\mathrm{Rr})$ of lignites is usually less than $0.5 \%$ [23], instead, there is huminite maceral groups in lignites. The random reflectance measured in oil of huminite is between $0.2-0.4 \% \mathrm{Rr}$ and is defined only for lignite [24].

Huminites is characterised by relatively high oxygen (18-28\%) and low carbon contents (60-77\%) compared with liptinite and inertinite. Huminite is coalified woody tissue derived from stems, roots, and vascular tissues of leaves. Textinite, ulminite, attrinite, densinite, corpohuminite and gelinite are macerals of huminite maceral group [24]. Liptinite maceral group is derived from resins, cuticles, spores, pollens and algal 
Chapter 2

remains. Inertinite group is derived from charring plant tissue caused by fire or intensive chemical degradation induced by micro-organism [22].

\subsection{Mineral Matter in coal}

Mineral matter in coal can be generalised as all elements present except organically derived or bound $\mathrm{C}, \mathrm{H}, \mathrm{N}, \mathrm{O}$, and $\mathrm{S}$ [22], although some researcher term the non macerals present in the coal as inorganic matters. Inorganic matters in coal include minerals and non-mineral inorganics [21]. Non-mineral inorganics include dissolved salts and other inorganic compounds in the coal's pore water as well as inorganic elements incorporated in the coal organic matrix, whereas mineral is defined as crystalline or non-crystaline discrete inorganic grain within coal organic matrix such as quartz and kaolinite [20]. If macerals contribute to the value of the coal in its utilisation processes, inorganic matters are mostly associated with problems arising during coal utilisations. Ash residue produced from burning the coal, slag from metallurgical processing, abrasion, corrosion, stickiness and pollution are some of the problems caused by inorganic matters in coal. Therefore, most coal conversion processes have a certain limit of the amount or composition of inorganic matters, in order to optimise the performance of those processes.

Most mineral grains have an average diameter of $20 \mu \mathrm{m}$ with the exception of pyrite and marcasite which have larger sizes. Some of the minerals concentrated in layer which can be cleaned by crushing or cleaning process. However, components which embedded in the coal matrix such as pyrite framboids and kaolinite will remain in the cleaned coal. From academic point of view, mineral is studied to reveal the formation history of coal. Syngenetic minerals are embedded during the early coalification process, while 
Chapter 2

epigenetic minerals, e.g. cleat fillings, are emplaced after the coalification process is complete [22].

Mineral grains are found in coal with different concentration; major, minor and trace. A major mineral in the coal is the mineral with concentration $>10 \mathrm{wt} \%$ of the low temperature ash (LTA) of the coal, minor mineral is if the LTA content between 1-10 $\mathrm{wt} \%$, while trace mineral is when $\mathrm{LTA}<1 \mathrm{wt} \%$ [22]. Major minerals consist of silicates which present as clay minerals, e.g. kaolinite $\left(\mathrm{Al}_{2} \mathrm{Si}_{2} \mathrm{O}_{5}(\mathrm{OH})_{4}\right)$, Illite $\left(\mathrm{KAl}_{2}\left(\mathrm{Si}_{3} \mathrm{Al}\right) \mathrm{O}_{10}(\mathrm{OH})_{2}\right)$, chlorite $(\mathrm{MgFeAl})_{6}\left(\mathrm{SiAl}_{4} \mathrm{O}_{10}(\mathrm{OH})_{8}\right)$, and also quartz $\left(\mathrm{SiO}_{2}\right)$. Minor minerals are carbonates, disulphides, sulphates, feldspars and sulphides. Dolomite $\left(\mathrm{Ca}, \mathrm{Mg}\left(\mathrm{CO}_{3}\right)_{2}\right)$, ankerite $\left(\mathrm{Ca}(\mathrm{FeMg}) \mathrm{CO}_{3}\right)$ and siderite $\left(\mathrm{FeCO}_{3}\right)$ are carbonates. Pyrite $\left(\mathrm{FeS}_{2}\right.$, cubic) and marcasite $\left(\mathrm{FeS}_{2}\right.$, orthorhombic) are disulphides. Sulphate minerals are gypsum $\left(\mathrm{CaSO}_{4} \cdot 2 \mathrm{H}_{2} \mathrm{O}\right)$, bassanite $\left(\mathrm{CaSO}_{4} \cdot 1 / 2 \mathrm{H}_{2} \mathrm{O}\right)$, anhydrite $\left(\mathrm{CaSO}_{4}\right)$ and jarosite $\left(\mathrm{KFe}_{2}\left(\mathrm{SO}_{4}\right)_{2}(\mathrm{OH})_{6}\right.$. Feldspars consist of plagioclase $((\mathrm{NaCa})$ $\left.\mathrm{Al}(\mathrm{AlSi}) \mathrm{Si}_{2} \mathrm{O}_{8}\right)$ and orthoclase $\left(\mathrm{KAlSi}_{3} \mathrm{O}_{8}\right)$. Galena $(\mathrm{PbS})$, pyrrothite $(\mathrm{FeS})$ and sphalerite $(\mathrm{ZnS})$ are classified as sulphides. Trace minerals include arsenopyrite (AsFeS), gold $(\mathrm{Au})$, magnetite $\left(\mathrm{Fe}_{3} \mathrm{O}_{4}\right)$, halite $(\mathrm{NaCl})$, mica, and hematite $\left(\mathrm{Fe}_{2} \mathrm{O}_{3}\right)$ [22, $25]$.

Minerals in coal are determined by many ways, such as high temperature ashing (HTA), low temperature ashing (LTA) and microscopy techniques. HTA is conventionally generated by combusting the sample under oxygen rich atmosphere at $1023 \mathrm{~K}$, then the minerals in the ash is characterised by $\mathrm{X}$ ray powder diffraction (XRD) analysis. To prevent some minerals undergo physical and chemical changes, HTA has been conducted at $643 \mathrm{~K}$ [26]. Even though HTA is performed at this temperature some minerals, such as pyrite, siderite and clay minerals experience irreversible changes of mass and crystal structure [26]. Minimum thermal alteration of the mineral matter in 
Chapter 2

coal can be achieved by low temperature ashing (LTA) which is obtained by controlled oxidation of coal samples in rf-induced plasma at 413-423 K [27], the ash produced is then characterised by XRD. The LTA is a good estimator of true ash content especially for high rank coal. In lower rank coals, however, direct LTA of the coal sample is less useful, since non-mineral inorganics form a significant part of the total mineral. During the LTA process, an interaction between non-mineral inorganics and organic sulphurs may occur to form calcium sulphate, iron sulphate or ammonium sulphate. These sulphates represent artefacts of the low temperature ashing, not constituents really occurring in the mineral forms of the coal. Therefore, LTA applied to leached lower rank coal residues after having a selective leaching of the non mineral inorganics usually provides a better assessment of mineral matters in lower rank coal [20]. Computer-controlled scanning electron microscopy (CCSEM) and electron microprobe analysis are the microscopy techniques commonly used to investigate inorganic matters in coals. CCSEM can be used on polished sections or on broken coal samples, while electron microprobe techniques may be applied to polished coal sections $[20,28]$. Fourier-transform infrared (FTIR) spectroscopy has also been used to assess minerals in coal [29].

Non-mineral inorganics present significantly in the inorganic matter of lower rank coals such as lignites and sub-bituminous coal. These inorganics greatly contribute to ash formation in lower rank coals, up to $30 \%$ of the ash produced from Australian brown coals is derived from non-mineral inorganics [20]. In some lignites, for example, the Victorian brown coal, non-mineral inorganics present as carboxylates and salts dissolved in the bed moisture. The inorganics $\left(\mathrm{Na}^{+}, \mathrm{Ca}^{2+}, \mathrm{Mg}^{2+}, \mathrm{Al}^{3+}\right.$ etc. $)$ are bonded to the $-\mathrm{COO}^{-}$groups as part of the coal organic matter. $\mathrm{NaCl}$ in Victorian brown coal is also dissolved to the bed moisture, when the lignites lost the moisture, the salt would interact with coal organic matter [21]. Non-mineral inorganics may be removed from 
Chapter 2

coal by an appropriate leaching process. Water soluble inorganics can be removed by water washing; carboxylates can be washed by soaking the coal in an ammonium acetate solution; while acid soluble inorganics may be treated by acid washing in a hydrochloric acid solution [20]. The inorganic elements in respective leachates can then be analysed, for example, using ion chromatograph (IC). The coal remaining after the leaching process can be subjected to low temperature ashing to identify the acid insoluble minerals present in the coal.

\section{5 Sulphur in Coal}

Almost all coals contain sulphur which varies between 0.2 and $11 \%$, in the forms of inorganic and organic sulphurs as well as elemental sulphur [4, 30]. Pyrite and Sulphate are the common forms of inorganic sulphur. The organic sulphurs exist as aliphatic, aromatic and heterocyclic forms [31]. The gaseous sulphur evolved during pyrolysis is a result of the conversion of different sulphur compounds in the coal $[4,5,32]$. Therefore understanding sulphur forms in the coal is very important in order to study sulphur transformation during coal pyrolysis and the following sections will review the type of sulphur in coal.

\subsubsection{Inorganic sulphur}

\subsubsection{Pyritic sulphur}

Pyrite is the main inorganic sulphur in coal and most of the sulphur forms in coal present as pyrite [4], although some coals contain very low pyritic sulphur, such as coals from Spain, India and New Zealand [31]. Pyrite was formed either at early diagenetic stage or late diagenetic stage [33]. During early diagenesis, sulphate which is reduced by bacterial reacted with organic matter to form $\mathrm{H}_{2} \mathrm{~S}$. Then $\mathrm{H}_{2} \mathrm{~S}$ reacted with iron $(\mathrm{Fe})$ 
Chapter 2

minerals to produce pyrite $[33,34]$. The pyrite formed during this stage is fine grained in micron sized which is present as crystals and framboids [33]. In late diagenesis, organic sulphur and sulphate may react with Fe minerals to form non framboidal granular to massive aggregate pyrite [33].

Table 2.1 Characteristics of sulphide minerals [35]

\begin{tabular}{|c|c|c|c|c|c|}
\hline Characteristics & Pyrite & Marcasite & Sphalerite & Galena & Chalcopyrite \\
\hline Formula & $\mathrm{FeS}_{2}$ & $\mathrm{FeS}_{2}$ & $\mathrm{ZnS}$ & $\mathrm{PbS}$ & $\mathrm{CuFeS}_{2}$ \\
\hline $\begin{array}{l}\text { Formula } \\
\text { weight }\end{array}$ & 119.97 & 119.97 & 97.44 & 239.2 & 183.5 \\
\hline $\begin{array}{l}\text { Melting point, } \\
\mathrm{K}\end{array}$ & 1465 & 723 & 1293 & 1393 & - \\
\hline Solubility & 0.0005 & 0.00049 & - & 0.00009 & - \\
\hline Colour & $\begin{array}{l}\text { pale brass } \\
\text { yellow }\end{array}$ & $\begin{array}{l}\text { brassy } \\
\text { yellow }\end{array}$ & $\begin{array}{l}\text { usually } \\
\text { black }\end{array}$ & $\begin{array}{l}\text { lead to } \\
\text { silver grey }\end{array}$ & $\begin{array}{l}\text { brassy } \\
\text { yellow }\end{array}$ \\
\hline Lustre & metallic & Metallic & $\begin{array}{l}\text { sub } \\
\text { metallic }\end{array}$ & metallic & metallic \\
\hline Transparency & opaque & Opaque & $\begin{array}{l}\text { transparent } \\
\text { to } \\
\text { translucent }\end{array}$ & opaque & opaque \\
\hline crystallography & $\begin{array}{l}\text { Isometric } \\
\text { (cubic) }\end{array}$ & orthorhombic & isometric & isometric & tetragonal \\
\hline Hardness & $6-6.5$ & $6-6.5$ & $3.5-4$ & $2.5+$ & $3.5-4$ \\
\hline $\begin{array}{l}\text { Specific } \\
\text { gravity }\end{array}$ & 5.018 & 4.8 & 4 & $7.5+$ & 4.2 \\
\hline
\end{tabular}

In addition to pyrite, marcasite $\left(\mathrm{FeS}_{2}\right)$, a different morphological form of pyrite, sphalerite $(\mathrm{ZnS})$, galena $(\mathrm{PbS})$ and chalcopyrite $\left(\mathrm{CuFeS}_{2}\right)$ may also be found in coal. The sulphides of $\mathrm{Ag}, \mathrm{As}, \mathrm{Bi}, \mathrm{Hg}, \mathrm{Co}, \mathrm{Ni}$ and $\mathrm{Sb}$ also have been identified [33]. Although these sulphide minerals are usually in trace concentrations, they can be found 
Chapter 2

in significant concentrations in some coals [36]. The characteristics of pure pyrite, marcasit, sphalerite, galena and chalcopyrite are described in Table 2.1.

\subsubsection{Sulphate sulphur}

The amount of sulphate sulphur in some coals is around $0.1 \%$ and sometimes negligible, however in weathered coals the sulphate sulphur content is quite significant relative to sulphites [4]. In some coals with low pyrite and high organic sulphur, the organic sulphur can oxidise to form calcium sulphate which also can be introduced by ground water[37]. Calcium sulphate, $\mathrm{CaSO} 4$, is the most common sulphate sulphur found in coal. Other sulphates such as, zinc sulphate $\left(\mathrm{ZnSO}_{4}\right)$, iron(II) sulphate $\left(\mathrm{FeSO}_{4}\right)$ and iron (III) sulphate, are also present in some coals [38]. Physical properties of pure sulphate sulphurs are described in Table 2.2.

Table 2.2 Characteristics of sulphates [35]

\begin{tabular}{|l|l|l|l|l|}
\hline Characteristics & $\begin{array}{l}\text { Calcium } \\
\text { sulphate }\end{array}$ & Zinc sulphate & $\begin{array}{l}\text { Iron(II) } \\
\text { sulphate }\end{array}$ & $\begin{array}{l}\text { Iron } \\
\text { sulphate }\end{array}$ \\
\hline Formula & $\mathrm{CaSO}_{4}$ & $\mathrm{ZnSO}{ }_{4}$ & FeSO & \\
\hline Formula weight & 136.14 & 161.44 & 278.02 & $\mathrm{Fe}_{2}\left(\mathrm{SO}_{4}\right)_{3} \cdot \mathrm{xH}_{2} \mathrm{O}$ \\
\hline Melting point, $\mathrm{K}$ & 1723 & 1013 & 337 & 562.02 \\
\hline Solubility & 0.298 & 42 & 32.8 & - \\
\hline Colour & white & white & $\begin{array}{l}\text { blue-green, } \\
\text { black }\end{array}$ & yellow \\
\hline Crystal habit & $\begin{array}{l}\text { rhombic or } \\
\text { monoclinic }\end{array}$ & rhombic & trigonal & Trigonal \\
\hline Refractive index & 1.576 or 1.5 & 1.669 & - & - \\
\hline Specific gravity & 2.96 & 3.74 & 1.89 & 2.1 \\
\hline
\end{tabular}




\subsubsection{Organic Sulphurs}

The amount of organic sulphur in coal is usually between $30-50 \%$ of the total sulphur in the coal [4]. The organic sulphur is originated from sulphur in original vegetations and also from the very complex reaction between organic molecules with inorganic sulphur introduced by sea water during the early diagenesis [13, 37]. The organic sulphur is unevenly distributed among macerals; vitrinite (huminite) and liptinite usually have higher concentration of organic sulphur, whereas inertinite contains less organic sulphur [37].

Organic sulphur in coal is a term for organic sulphur-containing functional groups present as part of the macromolecule structures of the coal. The exact groups occur in coal are very difficult to be identified, but a deduction about their forms can be made [4, 31, 39]. Destructive and non-destructive methods have been used to identify organic sulphur functionalities in coal $[4,36,38,40]$. All destructive methods for analysing sulphur functionalities in coal rely on a suitable organic sulphur model compound.

Organic sulphur in coal exist in various functional groups, for example thiolic (R-S-H), sulphidic (R-S-R'), disulphidic (R-S-S-R), sulphoxide (R-S-O-R) and thiophenic (heterocyclic) structures $[4,31,37]$. The aromaticity of the coal increases with the increase in coal rank. In higher rank coal some of the aliphatic thiols (labile structure) are restructured into heterocyclic structure as thyophenic groups (stabile compound). The amount of thiol and disulphide sulphurs is usually high in lignite, whereas thiophene is present substantially in high-rank coals. The lower the coal rank, the higher the labile sulphur contents $[4,5,37]$.

Some of the organic sulphur compounds which are commonly found in coal are summarized in Table 2.3 
Table 2.3 Organic sulphur model compounds

\begin{tabular}{|c|c|c|c|c|c|}
\hline \multirow[t]{2}{*}{$\begin{array}{l}\text { Sulphur } \\
\text { compound }\end{array}$} & \multirow[t]{2}{*}{ Structure } & \multicolumn{2}{|c|}{$\begin{array}{l}\text { Physical } \\
\text { propersties }\end{array}$} & \multicolumn{2}{|c|}{$\begin{array}{l}\text { Bond dissociation } \\
\text { Energies }\left(\mathrm{kJ} \cdot \mathrm{mol}^{-1}\right)\end{array}$} \\
\hline & & $\begin{array}{l}\text { MP, } \\
\text { K }\end{array}$ & $\begin{array}{l}\mathrm{BP}, \\
\mathrm{K}\end{array}$ & C-S & S-S \\
\hline $\begin{array}{l}\text { 2- } \\
\text { naphthalene } \\
\text { thiol }\end{array}$ & & $\begin{array}{l}353- \\
354\end{array}$ & $\begin{array}{l}365- \\
367\end{array}$ & 297.74 & \\
\hline $\begin{array}{l}\text { Dodecyl } \\
\text { benzene } \\
\text { sulphonic } \\
\text { acid }\end{array}$ & & & & 342.09 & \\
\hline $\begin{array}{l}\text { Benzyl } \\
\text { sulphide }\end{array}$ & & 322 & & 258.20 & \\
\hline $\begin{array}{l}\text { Dibenzyl } \\
\text { disulphide }\end{array}$ & & & & 266.83 & 277.86 \\
\hline $\begin{array}{l}\text { P-tolyl } \\
\text { disulphide }\end{array}$ & & 316 & & 311.84 & 275.79 \\
\hline $\begin{array}{l}\text { Dibenzo } \\
\text { thiophene }\end{array}$ & & 370 & 605 & 308.96 & \\
\hline
\end{tabular}




\subsection{Analysis of Sulphur in Coal}

The most common method to determine the concentration of total sulphur and proportions of the inorganic and organic sulphur forms in coal is wet chemistry method following the ASTM method. The ASTM standard test method, D2492 [41], applies to the determination of sulphate sulphur and pyritic sulphur in coal; the organic Sulphur is calculated by difference from the total sulphur obtained by another ASTM standard test method, D3177 [42].

Another chemical method to determine the sulphur concentration in the coal is to use elemental analyser, for example, a Carbon-Sulphur (CS) analyser. This instrument measures the total concentration of sulphur presents in the coal sample. To determine organic sulphur, the coal sample is washed using acid solution to removed inorganic sulphur. The residue, free of inorganic sulphur, was then analysed for its total sulphur, which is considered to be the organic sulphur as all inorganic sulphur would have been removed during the acid-washing. Inorganic sulphur in a sample was determined by the difference between the total sulphur and organic sulphur [43].

Determination of sulphur using chemical method is subjected to criticism. The main problem is obvious; determination of organic sulphur is by difference. Therefore, the organic sulphur value is subject to the accumulated errors in the sulphate and pyrite determinations. There is also the additional complication that the total sulphur and the sulphur forms have to be determined on different samples [44].

More recent development of electron microscopic instrumental techniques has enabled direct determination of organic sulphur in coal. There are reports in the literature on the use of scanning electron microscopy (SEM), electron probe microanalysis (EPM) and transmission electron microscopy (TEM). 
Chapter 2

The electron probe microanalysis (EPM) obtains images from the impingement of finely focused electron beam, generated at $15-20 \mathrm{eV}$, on polished samples mounted in an epoxy resin base. Quantitative analysis by EPM is performed by comparing the X-ray intensity produced by the sample with that produced by a standard of known composition. The potential advantages of EPM are that it is direct; measurements on individual macerals can be made in situ, non-destructive analysis and rapid. However, EPM suffered from problems associated with the standards used [44].

Scanning electron microscopy (SEM) produces three principal types of imaging according to the energy output of the beam. These include (i) secondary imaging, (ii) backscattered imaging and (iii) elementary X-ray maps. For all imaging modes, the energy and intensity of the emitted electron and X-ray is specific to the particular atom and the amount of the atom. Therefore SEM combined with EDX (energy dispersive analysis with X-ray Spectroscopy), SEM-EDX, is a useful tool to identify the mineral constituents and their distributions in coal. Olivella [45] found that the organic sulphur value from the ASTM result is almost the same as the result from SEM-EDX except for the coal samples with high inorganic sulphur contents. The presence of calcium sulphate or pyritic sulphur in coal matrix probably overestimates the organic sulphur concentrations, since it is difficult to find a 'clean' region during analysis.

Transmission electron microscopy (TEM) employs very thin specimens (much less than $1 \mu \mathrm{m}$ thick) and relies on the measurement of the sulphur K $\alpha \mathrm{X}$-ray line. The real strength of TEM lies in its ability to examine the spatial variation of organic sulphur in a coal sample [44].

The ASTM standard test method has a disadvantage of not being able to determine organic sulphur directly. On the other hand, EPM, SEM-EDX and TEM offer direct and 
non-destructive quantification of organic sulphur in coal. However, the accuracy of EPM, SEM-EDX and TEM measurements have only been justified by comparing results with the ASTM procedure and attaining of a good level of agreement between them [44]. This does not provide completely valid confirmation.

The methods presented above cannot determine the functionalities of organic sulphurs in coal. There are two methods to identify sulphur distributions in coal, i.e., (1) destructive method, for example, the temperature programmed reduction (TPR) and the temperature programmed oxidation (TPO), (2) non-destructive method, such as, X-ray absorption near-edge spectroscopy (XANES) and X-ray photoelectron spectroscopy (XPS). Stefanova et al [46] have studied the sulphur functionalities in the Bulgarian lignite using the atmosphere pressure temperature programmed reduction (AP-TPR) and the atmospheric pressure temperature-programmed oxidation (AP-TPO). Because the samples were very complex, in addition to potentiometric detection, they also used mass-spectrometer (MS), and 'off-line' gas-chromatograph/mass-spectrometer (GC/MS). To identify the sulphur functionalities, they used $\mathrm{H}_{2} \mathrm{~S}$ profiles from AP-TPRMS. They found that the lignite contained all types of sulphur compounds from aliphatic sulphides, aromatic sulphides, simple thiophenic structure and complex thiophenic structure. However, the information about at what temperatures each structure decomposes is not very clear. Furthermore, the effects of other gaseous sulphur evolved during the process on the determination of sulphur functionalities are vague. In the previous study of this Bulgarian lignite using AP-TPR, Maes et al [47] have determined at which particular temperatures each organic sulphur functionality evolves during coal utilisation processes. However, again, they determined the sulphur functionalities based on the $\mathrm{H}_{2} \mathrm{~S}$ profiles and did not include the effect of other gaseous sulphurs evolved. Using the controlled-atmosphere-programmed-temperature-oxidation (CAPTO), Miura et al [32] have quantified the sulphur functionalities in coal. They used $\mathrm{SO}_{2}$ profiles to 
Chapter 2

determine the sulphur distributions. They concluded that their results were quite similar to the sulphur functionalities distributions determined using XANES and XPS, however, they did not take into account the COS detected during their experiments.

The non-destructive techniques are techniques directly used to determine the sulphur functionalities in coal. XANES studies have been reported $[45,47-50]$ to be able to assess sulphur functionalities in coal. The XANES spectra are de-convoluted into peaks to obtain the composition of various sulphur functionalities in coal. However, XANES cannot differentiate between organic ester sulphate and inorganic sulphate. Furthermore, the reduced sulphur forms may be underestimated because the spectral response of reduced forms are lower than oxidised sulphur forms [45]. Another non-destructive technique is XPS. XPS can distinguish between reduced and oxidised sulphur forms easily. However, this technique is a surface technique, therefore, XPS may overestimates the amount of small particles in the large particles of coal [45]. It is worth to note that, although XANES and XPS may provide more accurate data to identify sulphur functionalities in coal, these techniques are not suitable for routine procedures.

\subsection{Sulphur behaviour during coal pyrolysis}

During pyrolysis, some sulphur compounds in coal are transformed to gas, liquid (tar) and solid (char) compounds. The change in sulphur distribution in the gaseous, char, and tar products may clarify the decomposition mechanism of each sulphur form. This section will review the behaviour of each type of sulphur during pyrolysis. 


\subsubsection{Pyrite transformation during coal pyrolysis}

Pure pyrite will decompose to form iron sulphide of lower sulphur content which is known as pyrrothite $\left(\mathrm{FeS}_{\mathrm{x}}, 1 \leq \mathrm{x}<2\right)$ and release sulphur gas when heated under inert atmosphere [51]. The thermal decomposition of pyrite can be written as follows:

$$
\mathrm{FeS}_{2} \leftrightarrow \mathrm{FeS}_{x(S)}+(1-0.5 x) \mathrm{S}_{2(g)}
$$

In a nitrogen atmosphere, thermal decomposition of pyrite starts at $793 \mathrm{~K}$ to form $\mathrm{FeS}_{\mathrm{x}}$ and then forms FeS at $1223 \mathrm{~K}$ [7]. The maximum temperature for decomposition of pyrite to form pyrrothite is at $1015 \pm 1 \mathrm{~K}$ and 1 atmosphere. This temperature is the incongruent melting point of pyrite or pyrite maximum stability [52]. Above this maximum stability, pyrrothite coexists with sulphur rich liquid until $1356 \mathrm{~K}$ when pyrrothite reacts with the liquid to form iron rich sulphur. Pyrite melts congruently at about $1465 \mathrm{~K}$ [53].

Under hydrogen atmosphere, pyrite reacts with hydrogen according to the following equation:

$$
\begin{aligned}
& \mathrm{FeS}_{2}+x \mathrm{H}_{2} \rightarrow \mathrm{FeS}_{(2-x)}+x \mathrm{H}_{2} \mathrm{~S} \\
& \mathrm{FeS}_{(2-x)} \rightarrow \mathrm{FeS}_{x}+2 / n(1-x) S_{n}
\end{aligned}
$$

The initial decomposition of pyrite under hydrogen atmosphere occurs at around $623 \mathrm{~K}$, FeS is formed at $673-843 \mathrm{~K}$, and at $1223 \mathrm{~K}$ elemental iron $(\mathrm{Fe})$ is formed $[7,54]$.

In the presence of oxygen, pyrite will be oxidised to form various final products, such as hematite $\left(\mathrm{Fe}_{2} \mathrm{O}_{3}\right)$, magnetite $\left(\mathrm{Fe}_{3} \mathrm{O}_{4}\right)$, iron sulphate $\left(\mathrm{Fe}_{2}\left(\mathrm{SO}_{4}\right)_{3}, \mathrm{FeSO}_{4}\right)$ and sulphur dioxide $\left(\mathrm{SO}_{2}\right)$ depending on the temperature, oxygen concentration, flow rates and particle size [51]. The oxidation can take place in two ways, the first is direct oxidation 
of pyrite and the second is a two-step process which involves thermal decomposition as in an inert atmosphere and followed by oxidation of pyrrothite formed [51]. The direct oxidation can be described with the following equation :

$$
\begin{aligned}
& \mathrm{FeS}_{2}+5.5 \mathrm{O}_{2} \rightarrow \mathrm{Fe}_{2} \mathrm{O}_{3}+4 \mathrm{SO}_{2} \\
& 2 \mathrm{FeS}_{2}+7 \mathrm{O}_{2} \rightarrow \mathrm{Fe}_{2}\left(\mathrm{SO}_{4}\right)_{3}+\mathrm{SO}_{2} \\
& \mathrm{FeS}_{2}+3 \mathrm{O}_{2} \rightarrow \mathrm{FeSO}_{4}+\mathrm{SO}_{2} \\
& \mathrm{Fe}_{2}\left(\mathrm{SO}_{4}\right)_{3} \leftrightarrow \mathrm{Fe}_{2} \mathrm{O}_{3}+3 \mathrm{SO}_{3} \\
& 2 \mathrm{FeSO}_{4} \leftrightarrow \mathrm{Fe}_{2} \mathrm{O}_{3}+\mathrm{SO}_{3}+\mathrm{SO}_{2} \\
& \mathrm{SO}_{2}+0.5 \mathrm{O}_{2} \leftrightarrow \mathrm{SO}_{3}
\end{aligned}
$$

whereas the two steps process can be described as follows :

$$
\begin{aligned}
& \mathrm{FeS}_{2} \rightarrow \mathrm{FeS}_{x(S)}+(1-0.5 x) \mathrm{S}_{2(g)} \\
& 2 \mathrm{FeS}_{x(S)}+(1.5+2 x) \mathrm{O}_{2(g)} \rightarrow \mathrm{Fe}_{2} \mathrm{O}_{3(s)}+2 x \mathrm{SO}_{2(g)} \\
& 2 \mathrm{FeS}_{x(S)}+(3+2 x) \mathrm{O}_{2(g)} \rightarrow \mathrm{Fe}_{2}\left(\mathrm{SO}_{4}\right)_{3(s)}+(2 x-3) \mathrm{SO}_{2(g)} \\
& \mathrm{FeS}_{x(S)}+(1+x) \mathrm{O}_{2(g)} \rightarrow \mathrm{FeSO}_{4(s)}+(x-) \mathrm{SO}_{2(g)} \\
& 2 \mathrm{FeSO}_{4(s)} \leftrightarrow \mathrm{Fe}_{2} \mathrm{O}_{3(s)}+\mathrm{SO}_{3(g)}+\mathrm{SO}_{2(g)} \\
& \mathrm{Fe}_{2}\left(\mathrm{SO}_{4}\right)_{3(s)} \leftrightarrow \mathrm{Fe}_{2} \mathrm{O}_{3(s)}+3 \mathrm{SO}_{3(g)} \\
& \mathrm{SO}_{2(g)}+0.5 \mathrm{O}_{2} \leftrightarrow \mathrm{SO}_{3(g)}
\end{aligned}
$$

Attar [4] reported that reactions (4)-(6) and (10)-(13) occur at lower temperatures between $623-673 \mathrm{~K}$. The decomposition of iron sulphates occurs at $893 \mathrm{~K}$ and becomes very fast above $983 \mathrm{~K}$.

Pyrite also reacts with carbon monoxide which is very slow in the temperatures below $1073 \mathrm{~K}$, according to[4]:

$$
\mathrm{FeS}_{2}+\mathrm{CO} \rightarrow \mathrm{FeS}+\mathrm{COS}
$$


Carbon can react with pyrite and iron sulphide at temperatures above $1273 \mathrm{~K}$ according to [4]:

$$
\begin{aligned}
& \mathrm{FeS}_{2}+\mathrm{C} \rightarrow \mathrm{Fe}+\mathrm{CS}_{2} \\
& \mathrm{FeS}+\mathrm{C} \rightarrow 2 \mathrm{Fe}+\mathrm{CS}_{2}
\end{aligned}
$$

In the presence of many organic compounds, pyrite starts to decompose to pyrrothite at temperatures as low as 523-573 K [4]. Gryglewicz [6] studied the pyrite transformation during pyrolysis of a high sulphur Polish coal. He observed the pyrite retention in the char using a scanning electron microscope equipped with an energy dispersive X-ray spectroscope (SEM-EDS) by monitoring the iron-sulphur ratio. From the EDS spectra, it was concluded that pyrite started to decompose at $633 \mathrm{~K}$. At $973 \mathrm{~K}$ the conversion of pyrite into iron sulphide (FeS) is almost complete. Chen et al [7] studied the transformation of pyrite during pyrolysis and hydropyrolysis of Chinese coals. They reported that pyrite starts to decompose to pyrrothite at about $673 \mathrm{~K}$ in a nitrogen atmosphere and at about $523 \mathrm{~K}$ in a hydrogen atmosphere. Conversion of pyrite to ferrous sulphide is complete at about $873 \mathrm{~K}$ during coal pyrolysis under the hydrogen atmosphere [55]. The external hydrogen atmosphere takes a principal role for reducing pyrite in coal during pyrolysis. Chen et al [7] also found that organic sulphur in the chars increases above $723 \mathrm{~K}$. They postulated that during coal pyrolysis, pyrite decomposes to form FeS and sulphur. The nascent sulphur is very active. If there is plenty of hydrogen, it will tend to form $\mathrm{H}_{2} \mathrm{~S}$ and go into vapour phase. If the hydrogen is insufficient, the sulphur will react with organic matrix to form organic sulphur. However, the theory is still unclear. Since there may be another factor influencing the pyrite sulphur transfer during pyrolysis. 
The kinetics of thermal decomposition of pyrite has been studied by a number of researchers. The thermal decomposition of pyrite is an endothermic reaction and is often kinetically controlled [51]. Lambert et al [56] studied the kinetics and mechanism of thermal decomposition of pure pyrite in a TGA under inert atmosphere from 620-923 K in order to elucidate the wide variety of the activation energy reported in literature on this transformation. They found that in Argon, helium and vacuum atmosphere, pyrite decomposition follows reaction (1). The reaction follows the unreacted core model and is controlled by chemical reaction. By assuming the reaction to be of the first-order in the surface area of the unreacted core, the apparent activation energy is observed about $200 \mathrm{~kJ} . \mathrm{mol}^{-1}$. However in the presence of hydrogen, different reactions can take place and the findings are summarised as follows:

1. The reaction between pyrite and hydrogen to form pyrrothite as follows: $\mathrm{FeS}_{2}+\mathrm{zH}_{2} \rightarrow \mathrm{FeS}_{(2-z)}+\mathrm{zH}_{2} \mathrm{~S}$

The apparent activation energy of reaction (R2.20) is around $30 \mathrm{~kJ} \cdot \mathrm{mol}^{-1}$.

2. Once the pyrrothite layer has formed, sulphur is removed according to the following equation :

$\mathrm{FeS}_{2}+(x-y) \mathrm{H}_{2} \rightarrow \mathrm{FeS}_{y}+(x-y) \mathrm{H}_{2} \mathrm{~S}$

Reaction (R2.21) has an activation energy of approximately $90 \mathrm{~kJ} \cdot \mathrm{mol}^{-1}$.

3. Sulphur atoms combine to form sulphur gas molecules at the surface.

4. The desorption of sulphur gas $\mathrm{S}_{\mathrm{n}}$ as can be describe with the following equation :

$\mathrm{FeS}_{x} \rightarrow \mathrm{FeS}_{y}+1 / \mathrm{n}(1-y) S_{n}$

where $1<\mathrm{y}<\mathrm{x}$.

An apparent activation energy approximately $200 \mathrm{~kJ}^{\mathrm{mol}} \mathrm{m}^{-1}$ is observed from reaction $(\mathrm{R} 2.22)$. 
Chapter 2

Therefore, in the presence of hydrogen, the pyrite thermal decomposition is assumed to be controlled by step (1) at the beginning of the reaction because the pyrrothite layer is not yet formed, but by step (4) after the pyrrothite layer has been formed.

Other researchers [57] found that thermal decomposition of pure pyrite is controlled by heat transport through a gas film at initial stage and has activation energy of approximately $113 \mathrm{~kJ} \cdot \mathrm{mol}^{-1}$, whereas in the later stage the process is dominated by product layer diffusion with apparent activation energy of approximately $96 \mathrm{~kJ} \cdot \mathrm{mol}^{-1}$.

Borah et al [58] studied the effect of pyrite on the kinetics of coal pyrolysis in a TGA. They revealed that pyrite has negligible effect on devolatilisation when it presents in small amounts, however when pyrite presents in significant amount it decreases the reactivity of coal. Assuming the reaction follows pseudo-first order, they obtained activation energies for coal blended with pyrite samples to be between $36-38 \mathrm{~kJ} \mathrm{~mol}^{-1}$. By comparing the activation energy of coal without pyrite added and blended samples, they argued that higher activation energy in the blended samples due to higher energy needed to decompose pyrite in addition to coal organic matter.

The activation energy is an important kinetic parameter that indicates the significance of the influence of temperature on the reaction. The variation in the activation energy reported may be caused by the varying relative significance of the diffusion process under different reaction conditions. It may be also because of the controlling reaction step in multi-step reaction mechanism $[51,56,57]$. 


\subsubsection{Sulphate transformation during coal pyrolysis}

Sulphate does not present significantly in fresh coal, however in weathered coal the presence of sulphate sulphur becomes more significant [4]. Therefore, it is important to study sulphate decomposition during coal pyrolysis. Generally, thermal degradation of sulphate in inert atmosphere can be described as follows [59]:

$\mathrm{MeSO}_{4} \leftrightarrow \mathrm{MeO}+\mathrm{SO}_{3}$

$\mathrm{SO}_{3} \leftrightarrow \mathrm{SO}_{2}+1 / 2 \mathrm{O}_{2}$

here Me denotes the inherent metal in coal.

Mullens et al [38] studied thermal decomposition of sulphate model compounds using atmospheric-pressure temperature-programmed pyrolysis (AP TPR) coupled to a mass spectrometer (MS). The compounds were calcium sulphate, zinc sulphate, iron (II) sulphate and iron (III) sulphate. The decomposition of the sulphate under inert atmosphere follows reactions (23) and (24) producing $\mathrm{SO}_{2}$ as the sulphur gas. They found that $\mathrm{CaSO}_{4}$ is a very stable compound. In an inert atmosphere $\mathrm{CaSO}_{4}$ decomposes above $1273 \mathrm{~K}, \mathrm{ZnSO}_{4}$ decomposes at $843 \mathrm{~K}$ and both iron sulphates decompose at 743 $\mathrm{K}$.

In reducing (hydrogen) atmospheres, the thermal decomposition of sulphates produces $\mathrm{SO}_{2}$ and $\mathrm{H}_{2} \mathrm{~S}$ [38]. However, no hydrogen sulphide is detected during pyrolysis of calcium sulphate under hydrogen. Hydrogen sulphide and sulphur dioxide released during reductive pyrolysis of zinc sulphate can be explained as follows:

$$
\begin{aligned}
& 2 \mathrm{ZnSO}_{4}+4 \mathrm{H}_{2} \rightarrow \mathrm{ZnS} . \mathrm{ZnO}+\mathrm{SO}_{3}+4 \mathrm{H}_{2} \mathrm{O} \\
& \mathrm{SO}_{3} \leftrightarrow \mathrm{SO}_{2}+0.5 \mathrm{O}_{2} \\
& \mathrm{SO}_{2}+3 \mathrm{H}_{2} \rightarrow \mathrm{H}_{2} \mathrm{~S}+2 \mathrm{H}_{2} \mathrm{O} \\
& \text { Sulphur Transformation during Pyrolysis of an Australian Lignite }
\end{aligned}
$$


From the pattern of gaseous sulphur evolution of iron (II) sulphate and iron (III) sulphate, it can be concluded that in hydrogen atmosphere $\mathrm{Fe}_{2}\left(\mathrm{SO}_{4}\right)_{3}$ decomposes to form $\mathrm{FeSO}_{4}$ and $\mathrm{Fe}_{2} \mathrm{O}_{3}$. The decomposition of $\mathrm{Fe}_{2}\left(\mathrm{SO}_{4}\right)_{3}$ to $\mathrm{Fe}_{2} \mathrm{O}_{3}$ can be described as follows :

$\mathrm{Fe}_{2}\left(\mathrm{SO}_{4}\right)_{3} \leftrightarrow \mathrm{Fe}_{2} \mathrm{O}_{3}+3 \mathrm{SO}_{3}$

whereas the decomposition reaction of $\mathrm{FeSO}_{4}$ proceeds according to:

$$
\begin{aligned}
& \mathrm{FeSO}_{4} \rightarrow \mathrm{FeO}+\mathrm{SO}_{3} \\
& \mathrm{FeSO}_{4}+4 \mathrm{H}_{2} \rightarrow \mathrm{FeS}+4 \mathrm{H}_{2} \mathrm{O} \\
& \mathrm{FeS}+\mathrm{H}_{2} \rightarrow \mathrm{Fe}+\mathrm{H}_{2} \mathrm{~S} \\
& \mathrm{FeO}+\mathrm{H}_{2} \mathrm{~S} \rightarrow \mathrm{FeS}+\mathrm{H}_{2} \mathrm{O}
\end{aligned}
$$

When the residue from reductive pyrolysis of $\mathrm{Fe}_{2}\left(\mathrm{SO}_{4}\right)_{3}$ and $\mathrm{FeSO}_{4}$ was subjected to combustion, no $\mathrm{SO}_{2}$ gas could be detected, implying that all sulphur is released during the pyrolysis either as $\mathrm{SO}_{2}$ or $\mathrm{H}_{2} \mathrm{~S}$.

In the presence of organic matters, the decomposition of sulphates occur at lower temperatures [9]. They studied the thermal decomposition of $\mathrm{Fe}_{2}\left(\mathrm{SO}_{4}\right)_{3}$ and $\mathrm{ZnSO}_{4}$ impregnated on the char under helium atmosphere. $\mathrm{SO}_{2}$ is the only gaseous sulphur evolved during thermal decomposition of the two compounds. $\mathrm{Fe}_{2}\left(\mathrm{SO}_{4}\right)_{3}$ decomposes at $473 \mathrm{~K}$ and $\mathrm{ZnSO}_{4}$ reduces at $600 \mathrm{~K}$.

Ibara et al [60] studied the evolution of volatile sulphur during coal pyrolysis. He reported that the evolution of $\mathrm{SO}_{2}$ is directly related to the sulphate contents of coals. Attar [4] reported that sulphur dioxide can also be produced from the reaction between pyrite and calcium sulphate according to :

$$
\mathrm{CaSO}_{4}+2 \mathrm{FeS}_{2}+\mathrm{H}_{2} \mathrm{O} \rightarrow \mathrm{CaO}+\mathrm{FeS}+2 \mathrm{SO}_{2}+\mathrm{H}_{2}
$$


Chapter 2

Ibara et al [60] reported that iron sulphates present in weathered coals decompose at 573 $\mathrm{K}$ during pyrolysis. $\mathrm{CaSO}_{4}$ in the coals starts to decompose at $623 \mathrm{~K}$. They also observed the increased in organic sulphur in char during pyrolysis where the iron sulphates decomposition occurs. This suggests the incorporation of the released sulphur into char as organic sulphur. These researchers also noted that iron sulphates may convert to complex sulphide which is not determinable by chemical analysis, therefore, giving inaccuracy in determination of organic sulphur in the char.

The amount of volatile sulphur released from the decomposition of sulphate depends on the volatility of the compound [61]. From Table 2 it can be seen that $\mathrm{CaSO}_{4}$ is less volatile than $\mathrm{FeSO}_{4}$. A greater amount of $\mathrm{FeSO}_{4}$ can be converted to the gas phase during thermal decomposition than $\mathrm{CaSO}_{4}$. Therefore, the residual sulphate found in the char is usually in the form of calcium sulphate.

\subsubsection{Organic sulphur transformation during coal pyrolysis}

In order to get a better understanding on the sulphur transformation during coal pyrolysis, it is necessary to study the behaviour of organic sulphur using model compounds $[38,62]$. Although it is uncertain whether organic sulphur in coal would behave similarly to model compounds of organic sulphur, studying organic sulphur model compounds will give a basic knowledge on the assignment of the temperature region in which a certain type of sulphur is reduced or hydrogenated [38].

Mullens et al [38] studied the behaviour of model compounds of organic sulphur immobilised on silica using the atmospheric pressure-temperature programmed pyrolysis coupled with a mass spectrometer (AP-TPR-MS). They clarified the mechanisms behind the low reduction efficiencies of thiophenic and oxidised model compounds to $\mathrm{H}_{2} \mathrm{~S}$. However, to achieve a quantitative sulphur balance needs a more 
Chapter 2

sensitive detection instrument. In addition, even though the sulphur-containing compounds immobilised on silica are stable, the interactions between the inert silica matrix and the sulphur model compounds are different from those between a coal matrix and the sulphur-containing structures. Xu et al [62] studied the behaviour of sulphur model compounds impregnated on a charcoal to simulate coal-like environment in a temperature-programmed decomposition coupled with a mass spectrometer (TPD-MS). They revealed that the key intermediate for volatile sulphur emission is the formation of sulphur radical.

Yperman et al [63] and Maes et al [47] studied organic sulphur transformation during coal pyrolysis in atmospheric-pressure temperature-programmed reduction (AP TPR). The decomposition of organic sulphur depends on the complexity of the organic sulphur. Based on the evolution of gaseous sulphur, they reported that thiols and aliphatic sulphide start to decompose as low as $518 \mathrm{~K}$, aliphatic disulphides and mixed aliphatic-aromatic sulphides decompose at approximately $713 \mathrm{~K}$, aromatic sulphides and disulphides reduction occurs approximately at $883 \mathrm{~K}$, and thiophenes are reduced above $973 \mathrm{~K}$.

To get a better understanding of the organic sulphur during coal pyrolysis, it is important to compare the organic sulphur transformation in coal with the transformation of organic sulphur model compounds impregnated in free-sulphur coal. 


\subsection{Effect of inorganics on Sulphur Transformation}

Many studies have been reported on the influence of inorganics in coal to the sulphur transformation during coal utilisation. Alkali and alkaline earth inorganics present significantly in some low rank coals such as Victorian brown coal[64, 65]. Cations such as $\mathrm{Ca}^{2+}, \mathrm{Mg}^{2+}$ and $\mathrm{Na}^{+}$are bonded to carboxylic groups as part of the macromolecular network of coal [66]. During pyrolysis, the carboxylates decompose at temperatures lower than 573 Kreleasing water $\left(\mathrm{H}_{2} \mathrm{O}\right)$ and carbon dioxide[66]. $\mathrm{Ca}$ and $\mathrm{Na}$ originally attached with carboxylates groups (-COO-) may be rebounded to char matrix (-CM) according to [67]:

$$
\begin{aligned}
& (-\mathrm{COO}-\mathrm{Ca}-\mathrm{COO}-)+(-\mathrm{CM}) \rightarrow(-\mathrm{COO}-\mathrm{Ca}-\mathrm{CM})+\mathrm{CO}_{2} \\
& (-\mathrm{COO}-\mathrm{Ca}-\mathrm{CM})+(-\mathrm{CM}) \rightarrow(\mathrm{CM}-\mathrm{Ca}-\mathrm{CM})+\mathrm{CO}_{2} \\
& (-\mathrm{COO}-\mathrm{Na})+(-\mathrm{CM}) \rightarrow(\mathrm{CM}-\mathrm{Na})+\mathrm{CO}_{2}
\end{aligned}
$$

It is possible that the newly formed $\mathrm{Ca}-\mathrm{CM}$ and $\mathrm{Na}-\mathrm{CM}$ will be broken again to release some $\mathrm{Ca}$ and $\mathrm{Na}$ as volatile.

Most of chlorine in the coals and part of $\mathrm{Na}$ in the coal are present as $\mathrm{NaCl}$ [68]. Quyn et al [69] reported that $\mathrm{NaCl}$ in the coal is decomposed into gases (probably $\mathrm{HCl}$ ) and char bonded $\mathrm{Na}(\mathrm{CM}-\mathrm{Na})$ at low temperature $(773 \mathrm{~K})$. The transformation of $\mathrm{NaCl}$ may be represented as [70]:

$$
\mathrm{NaCl}+\mathrm{CM}-\mathrm{H} \rightarrow \mathrm{CM}-\mathrm{Na}+\mathrm{HCl}
$$

Alkali and alkaline earth components may retain gaseous sulphur as solid compounds [71]. At Low temperature under oxidizing conditions, the alkaline components react with sulphur in the coal to produce alkaline sulphate, while under reducing atmosphere the reaction produces sulphides [72]. Anthony and Granatstein [73] stated that during 
Chapter 2

combustion by use calcium-based sorbent in fluidised bed combustors under oxidative conditions, the sulphation product below $723 \mathrm{~K}$ is $\mathrm{CaSO}_{3}$, then, between $923-973 \mathrm{~K}$, are $\mathrm{CaSO}_{3}$ and $\mathrm{CaSO}_{4}$, finally, at above $973 \mathrm{~K}, \mathrm{CaSO}_{4}$ is the sulphation product. On the other hand, the sulphation products under reductive conditions are $\mathrm{CaSO}_{3}, \mathrm{CaSO}_{4}$ and CaS below $1100 \mathrm{~K}$, whereas above this temperature the sulphation products are only $\mathrm{CaS}$ and $\mathrm{CaSO}_{4}$. Furthermore, they claimed that the route of the sulphation process has not been clear yet, since the mechanism of the formation of $\mathrm{CaSO}_{4}$ is still debatable, whether it is formed via reaction (R2.38) and (R2.39) or (R2.40) and (R2.41) as follows:

$$
\begin{aligned}
& \mathrm{CaO}+\mathrm{SO}_{2} \Leftrightarrow \mathrm{CaSO}_{3} \\
& \mathrm{CaSO}_{3}+1 / 2 \mathrm{O}_{2} \Leftrightarrow \mathrm{CaSO}_{4} \\
& \text { or } \\
& \mathrm{SO}_{2}+1 / 2 \mathrm{O}_{2} \Leftrightarrow \mathrm{SO}_{3} \\
& \mathrm{CaO}+\mathrm{SO}_{3} \Leftrightarrow \mathrm{CaSO}_{4}
\end{aligned}
$$

In addition, the presence of $\mathrm{CaSO}_{3}$ and $\mathrm{CaSO}_{4}$ under a reducing condition is questionable, as hydrogen sulphide is the main gaseous sulphur product under this condition.

It has been pointed out that calcium plays an important role in sulphur retention, while other components are limited [71]. However Telfer and Zhang [2] reported that water soluble inorganics (sodium) in a South Australian brown coal increased the retention of sulphur during pyrolysis between 673-773 K, whereas acid-soluble inorganics (calcium) initiated the decomposition of organic sulphur to form calcium sulphide in the char between 1073-1173 K. Another study showed that during the combustion of coal added with limestone, in general, as time progressed more $\mathrm{SO}_{2}$ was captured by limestone [28] . In the combustion of coal added with calcium acetate, however, it was found that the emitted sulphur being captured by a calcium acetate sorbent was more than by a 
Chapter 2

limestone sorbent, $80 \%$ and $60 \%$, respectively [74]. Even though, calcium plays a significant role in capturing sulphur containing gas, at above $1473 \mathrm{~K}$ the calcium-based component is not effective since calcium sulphate as a sulphation product decomposes completely and decreases the sulphur retention capability [71].

Calcium, strontium and barium are alkaline earth metal components. They have similar chemical and physical properties, including the capability to capture gaseous sulphur. Strontium sulphate and barium sulphate, the sulphation products of a strontioum-based sorbent and a barium-based sorbent, respectively, are more thermally stable than calcium sulphate, they decompose above $1853 \mathrm{~K}$ [71]. However, strontium and barium based sorbents are more expensive compared to calcium based sorbents. Other stable desulphurisation products at quite high temperatures are calcium aluminate sulphate, and calcium silicate sulphate [71]. Another study showed that calcium aluminosilicate having molar $\mathrm{Ca} / \mathrm{Si} \geq 1.0$ capable to capture gaseous sulphur during coal combustion [28]. It is, therefore, probably possible to blend limestone with other minerals or components that contain $\mathrm{Sr}, \mathrm{Ba}, \mathrm{Al}$ or $\mathrm{Si}$ to produce good sorbents to capture emitted sulphur from coal utilisation at high temperatures, whereas for low-temperature pyrolysis, water soluble inorganics such as $\mathrm{Na}$ might be used to retain sulphur in the char thus lower the evolution of gaseous sulphur to the environment.

\subsection{Mechanisms and reaction kinetics of sulphur transformation}

When coal is subjected to pyrolysis, it evolves volatiles with residue remaining as char, as can be described as follows :

$$
\text { Coal } \rightarrow \text { pyrolysis } \rightarrow \text { volatiles }+ \text { char }
$$


Solomon et al [75] described the changes in the structures of bituminous coal during pyrolysis. They concluded that coal pyrolysis proceeds in the following steps:

\section{Break of hydrogen bonds}

2. Diffusion and devolatilisation of 'guest molecules"

3. Formation of cross-links at low temperatures by evolving $\mathrm{H}_{2} \mathrm{O}$ and $\mathrm{CO}_{2}$

4. Change of macromolecules to radical fragments

5. Stabilisation of radical fragments

6. Diffusion and devolatilisation of molecules formed in 5

7. Recombination of radical formed in 5 to form larger molecules

8. Decomposition of functional groups to form light gases

9. Formation and growth of poly-aromatic structures accompanied by $\mathrm{H}_{2}$ formation at high temperature.

The pyrolysis of coal is therefore very complicated. Kinetic analysis of coal pyrolysis can be solved based on a simple global model to sophisticated models.

Using the conversion of solid reactant, $\mathrm{X}$, the pyrolysis rate is defined by the following equation [70] :

$$
\frac{d X}{d t}=k_{o} e^{-E / R T} f(X)
$$

where $\mathrm{dX} / \mathrm{dt}$ is the conversion rate, $\mathrm{T}$ is the temperature, $\mathrm{k}_{\mathrm{o}}$ is the frequency factor, $\mathrm{E}$ is the activation energy, $\mathrm{f}(\mathrm{X})$ is the change in the solid reactant with the progress of reaction. The above equation may be solved by using differential method as described by Freedman [76] or using integral method as proposed by Coats and Redfern [77]. 
Chapter 2

Coal pyrolysis can also be represented by the amount of volatile matter formed. By assuming that each volatile product is formed following a first order reaction, the formation rate of each volatile can be described as follows:

$d V_{i} / d t=k_{o i} \cdot \exp \left(-E_{i} / R T\right) \cdot\left(V_{i}^{*}-V_{i}\right)$

where $\mathrm{dV}_{\mathrm{i}} / \mathrm{dt}$ is the formation rate of volatile $\mathrm{i}, \mathrm{T}$ is the temperature, $\mathrm{k}_{\mathrm{oi}}$ is the frequency factor of component $i, E_{i}$ is the activation energy of component $i, V_{i}{ }^{*}$ is the ultimate amount of volatile $i$, and $V_{i}$ is the amount of volatile $i$ at $t$ [65].

By applying a single reaction model Garcia-Labiano [78] studied the kinetic of sulphur release during pyrolysis. The rate of production of sulphur species, (dVs/dt), representing the summation of all possible pathways of sulphur evolution was calculated by:

$(\mathrm{dVs} / \mathrm{dt})=\mathrm{k}^{\prime}\left(\mathrm{Vs}^{*}-\mathrm{Vs}\right)$

$\mathrm{k}^{\prime}=\mathrm{k} \cdot \exp (-\mathrm{Ea} / \mathrm{R} \cdot \mathrm{T})$

Where Vs is the concentration of sulphur compounds precursors in the solid, $\mathrm{Vs}^{*}$ is the maximum concentration of the precursors, $\mathrm{R}$ is the ideal gas constant and $\mathrm{k}$ and $\mathrm{E}$ are the Arrhenius parameters. The distribution of the total sulphur yield into gaseous sulphur products was determined experimentally from a percentage distribution of the individual sulphur forms. However, due to the small concentration of some gaseous sulphur products such as $\mathrm{CS}_{2}, \mathrm{COS}$ and $\mathrm{CH}_{3} \mathrm{SH}$, it was impossible to express a single reaction model for each individual sulphur species.

Another first order kinetics model of coal pyrolysis was proposed by Sugawara [79]. For the different heating rates, the model successfully simulated the sulphur 
Chapter 2

transformations during coal pyrolysis in a thermogravimetric analyzer (TGA) and a drop tube furnace (DTF) pyrolyser.

\subsection{Conclusions from the Literature Review}

Based on the above literature review, the following conclusions can be drawn:

Lignite is characterised as low rank coal which usually contains high moisture, ash and oxygen but has low carbon and calorific value. Lignites also have less aromatic carbon but higher aliphatic carbon structures than high rank coal.

Inorganic matters in coal include mineral and non-mineral inorganics. In lignites, nonmineral inorganics present significantly, thus it contributes greatly to the ash formation. Some of non-mineral inorganics present as carboxylates and salts dissolved in the bed moisture. Some problems, such as ash residue produced from burning the coal, slag from metallurgical processing, abrasion, corrosion, stickiness and pollution are caused by inorganic matters in coal.

Almost all coals contain sulphur in the form of pyrite, sulphate and organic sulphur. The organic sulphur compound in coal exists in a wide range of functionalities. Compared to high-rank coal, the organic sulphur functionalities in lignite is more labile structures, such as thiols and disulphides.

Even though the ASTM standard test method has been criticised, it remains the most reliable method in determining sulphur forms in coal, since, the results of instrumental analysis techniques (EPM, SEM-EDX and TEM) are always compared to the result from the ASTM method. The sulphur functionalities can be determined using a destructive method ( TPR or TPO) and/or a non-destructive method (XPS or XANES). 
Chapter 2

During coal pyrolysis sulphur in coal decomposes to evolve gases mainly $\mathrm{H}_{2} \mathrm{~S}$ with traces of $\mathrm{SO}_{2}, \mathrm{CS}_{2}, \mathrm{COS}, \mathrm{CH}_{3} \mathrm{SH}$. Pyrite starts to decompose at $663 \mathrm{~K}$ to release $\mathrm{H}_{2} \mathrm{~S}$ to the gas phase and also retain some of the sulphur in the char. Sulphate decomposes between $300-773 \mathrm{~K}$ to evolve $\mathrm{SO}_{2}$. Organic sulphur in coal decomposes over a wide range of temperatures, according to the complexity of the organic sulphur functionality. The decomposition results mainly in the release of $\mathrm{H}_{2} \mathrm{~S}$, but oxygenated organic sulphur may release $\mathrm{SO}_{2}$. The conversion of simple sulphur compounds to more complex organic species in the char also occurs during the pyrolysis. The thermal decomposition of the sulphur model compounds can be used to study the behaviour of sulphur transformation in coal.

Alkali and alkaline earth compounds which present significantly in lower rank coals affect the sulphur transformation during coal pyrolysis. Some of the alkali and alkaline earth compounds are reported to retain sulphur during coal utilisation processes.

First order kinetics models have been developed to predict the evolution of sulphur containing gases during pyrolysis.

\subsection{Objectives of the Current Studies}

The current study is designed to specifically address the environmental and processing concerns regarding the utilisation of Western Australian lignites which contain a significant amount of sulphur and inorganic matters. Thus the overall objective of this project is to contribute on the fundamental and scientific level to the development of a model for sulphur emissions.

Based on the literature review and the conclusions drawn, it is evident that pyrite, sulphate and organic sulphur transformations during coal pyrolysis require extensive 
Chapter 2

investigation. The development of a variety of experimental and analytical techniques in order to gain comprehensive and fundamental information regarding the mechanisms of sulphur transformations in lignites which contain high oxygen and high inorganic matters is therefore necessary. Investigation of sulphur transformations can be achieved by analysing the sulphur containing gases evolved during pyrolysis at various times and temperatures and also by analysing sulphur retains in the char. Pyrolysis of low-sulphur lignite blended with sulphur model compounds which are usually found in coal is also required to gain further information of the transformations of each sulphur compound in lignite. Finally, to understand the retention of sulphur by inorganic matter, specific investigation of the interaction of inherent and added inorganic material with sulphur must also be conducted. 


\section{CHAPTER 3}

\section{Methodology and Experimental Techniques}

\subsection{Introduction}

Several analytical and experimental techniques have been employed to characterise lignite samples and to investigate the sulphur transformation during lignite pyrolysis. Theses include (1) pre-treatment of the samples, (2) characterisation of lignite samples using various advanced microscopic techniques, (3) simulated pyrolysis in TGA and fixed-bed reactor and (4) investigation of the sulphur in solid and gas. This chapter presents in details these techniques and procedures.

\subsection{Lignite Sample and Sample Preparation}

Raw lignite samples employed in this study were Western Australian lignites with different inorganic contents. There were eleven (11) lignite samples used in the experiments, the lignites were named as Lignite 1 (L1), Lignite 2 (L2) to Lignite 11 (L11), respectively.

Some of the samples underwent through an acid-washing process which will be described in this section. To study the transformation of pyrite, sulphate and organic sulphurs during lignite pyrolysis, pure pyrite, pure sulphates and organic sulphur compounds were used and loaded into the acid washed L1. Inorganic matters were also loaded to acid washed L3 to study the effect of inorganic matter on the transformation of sulphur during lignite pyrolysis. The methods of loading sulphur compounds and inorganic matters will be described as well in the following sections. 


\subsubsection{Drying}

All samples employed in the pyrolysis experiments were dried samples. The drying was conducted in a vacuum drier at $333 \mathrm{~K}$ until the lignites were able to be ground. The ground lignites were then dried again in the vacuum oven at $333 \mathrm{~K}$ for 48 hours and the dried samples were stored in an air tight container. Prior to each pyrolysis experiment the moisture content of the sample was checked by a thermogravimetric analyser (TGA).

\subsubsection{Sieving}

The samples were pulverised and sieved into certain particle size ranges. The effect of particle size on the total sulphur content in the raw lignite was investigated before choosing the particle size range for pyrolysis.

Raw samples were ground to a particle size range of $<250 \mu \mathrm{m}$ before employed in acid washing.

\subsubsection{Acid washing}

Acid washed lignite was prepared to remove acid soluble inorganics such as carboxylate groups by washing the ground samples with a dilute hydrochloric acid ( $\mathrm{HCl})$. The acid washing procedure was as follows: about $30 \mathrm{~g}$ of lignite was dispersed in $150 \mathrm{ml} 2.5 \mathrm{M}$ $\mathrm{HCl}$. The mixture was heated to $353 \mathrm{~K}$ for 3 hours and cooled to room temperature and left overnight. The slurry was then filtered and washed with warm double-distilled water $(353 \mathrm{~K})$ until no chloride ion was detectable. The remaining solid then was dried in a vacuum oven at $333 \mathrm{~K}$ for 24 hours. 


\subsubsection{Pyrite Addition}

A pyrite mineral from Hunzala, Peru purchased from Mannum Minerals Australia was used in this study. The impurities found in the pyrite were $30 \mathrm{mg} \cdot \mathrm{kg}^{-1}$ of $\mathrm{Cl}$ and 8.3 mg.kg ${ }^{-1}$ of $\mathrm{F}$. The pyrite was ground to particle sizes $<150 \mu \mathrm{m}$.

Lignite and pyrite blends were prepared with L1. To blend the pyrite with lignite L1, the following procedures were performed [58]. A certain weight of the pyrite and acidwashed L1 were taken and mixed. It was then ground mechanically for about 2 hours. There were three lignite and pyrite blends prepared for this study, namely, 5P-95L1 (5\% pyrite $+95 \% \mathrm{~L} 1), 10 \mathrm{P}-90 \mathrm{~L} 1(10 \%$ pyrite $+90 \% \mathrm{~L} 1)$, and $50 \mathrm{P}-50 \mathrm{~L} 1(50 \%$ pyrite $+50 \%$ L1).

\subsubsection{Sulphate Sulphur Addition}

The sulphates used in the experiments were calcium sulphate anhydrite $\left(\mathrm{CaSO}_{4}\right)$, ferrous sulphate $\left(\mathrm{FeSO}_{4}\right)$ and ferric sulphate $\left(\mathrm{Fe}_{2}\left(\mathrm{SO}_{4}\right)_{3}\right) \cdot \mathrm{CaSO}_{4}$ was purchased from Labchem with $100 \%$ purity. $\mathrm{FeSO}_{4} \cdot 7 \mathrm{H}_{2} \mathrm{O}$ and $\mathrm{Fe}_{2}\left(\mathrm{SO}_{4}\right)_{3} \cdot \mathrm{xH}_{2} \mathrm{O}$ with $>99 \%$ purity was purchased from Sigma.

Calcium sulphate and ferrous sulphate were loaded to the acid washed lignite L1 by incipient wetness method [9]. About 1 gr of pure sulphates was dissolved in double distilled water. Then approximately 10 grams acid-washed L1 was added to the sulphate solution. The mixture was stirred and left overnight before dried in a vacuum oven at $333 \mathrm{~K}$ for 24 hours. 


\subsubsection{Organic Sulphur Addition}

The organic sulphurs chosen to use in the experiments were naphthalene thiol $\left(\mathrm{C}_{10} \mathrm{H}_{8} \mathrm{~S}\right)$ and benzyl sulphoxide $\left(\mathrm{C}_{14} \mathrm{H}_{14} \mathrm{OS}\right)$. The organic sulphurs were purchased from Sigma Aldrich.

Impregnation of organic sulphurs to the acid washed L1 was by incipient wetness method in a tetra hydro furan (THF) solution and the following methods were followed [9, 62]. Approximately 5 grams acid-washed L1 were mixed with 1 gram organic sulphur. The mixture was then dissolved in the THF and left overnight. It was then dried in a vacuum oven at $333 \mathrm{~K}$ for 24 hours. Since the particle size ranges changed after the impregnation process, the samples needed to be ground and sieve to a particle size range of $150-250 \mu \mathrm{m}$.

\subsubsection{Inorganic Matter Addition}

Kaolinite and sodium were chosen to be added to the acid lignite. Sodium acetate with $100 \%$ purity was purchased from Chem-supply. The sodium-added sample was prepared by mixing $30 \mathrm{~g}$ of the acid-washed $\mathrm{L} 3$ with $150 \mathrm{ml} 1 \mathrm{M}$ sodium acetate solution and stirring for 24 hours at room temperature. The mixture was then washed and filtered with double-distilled water to remove excess sodium ion. Finally, the remaining solid was dried in a vacuum drier at $333 \mathrm{~K}$ for 48 hours.

The kaolinite-added sample was prepared by mixing about 1 gram of ground kaolinite $(<150 \mu \mathrm{m})$ with about 10 gram of the acid-washed L3 and mixed thoroughly to ensure uniformity. 


\subsection{Lignite Characterisation}

A combination of techniques was used to characterise the raw lignite samples and their char products. XRD and XRF was used to identify the mineral phases and inorganic matter conttent present in lignite ash, SEM, FTIR and NMR were used to characterise the structure of the lignite, whereas the maceral was identified by petrographic analysis. The following sections will discuss in detail the methods to characterise lignite sample.

\subsubsection{Proximate, Ultimate, Sulphur Forms and Inorganic Forms analysis}

Proximate analysis was carried out to determine moisture, ash, volatile matter and fixed carbon contents in lignite. Proximate analysis, moisture and ash analysis was carried out according to HRL method 1.6 (Leco MAC) and volatile matter and fixed carbon according to Standards Australia method AS2434.2.

Ultimate analysis was carried out to determine $\mathrm{C}, \mathrm{H}, \mathrm{N}, \mathrm{S}, \mathrm{O}$ and mineral and inorganic contents in the samples. Ultimate analysis (Leco $\mathrm{CHN}$ ) and calorific values (Leco AC350) were also carried out according to AS1038.6.4 and AS 1038.5, respectively. Mineral and inorganic values were determined based on a combination of AS1038.14.1, AS2434.9, AS2434.6.1-part-8 and AS1038.8.2.

Sulphur forms (Leco SC32 sulphur determinator) were determined according to AS1038.11.

Chloride and sodium contents were analysed by High Performance Ion Chromatography (HPIC) using the Dionex ${ }^{\circledR}$ ICS-1000 ion chromatograph. Anions were separated using an $\mathrm{AS}-12 \mathrm{~A}$ and $\mathrm{AG}-12 \mathrm{~A}$ column set and cations were separated using a CS-12A 
Chapter 3

column. Solutions were diluted $200 \times$ prior to analysis for optimum determination of $\mathrm{Na}$ and Cl. Samples were prepared for water soluble chloride and sodium and water insoluble chloride analysis by first grounding to a powder in an agate mortar and mixing about $1 \mathrm{~g}$ of each dried sample in $20 \mathrm{~mL}$ of deionised and deoxygenated water and then shaking the mixture for 6 hours. Solutions were then filtered prior to HPIC.

\subsubsection{Low temperature ashing (LTA) and High temperature ashing (HTA)}

Lignite samples were ashed at low temperature (423-473 K) and high temperature (723 $\mathrm{K}, 823 \mathrm{~K}$ and $923 \mathrm{~K}$ ). Low temperature ashing (LTA) was carried out in an Emitech K1050X oxygen plasma asher working at a microwave power of $150 \mathrm{~W}$. Approximately $1 \mathrm{~g}$ of the sample was spread out on Petri dishes and exposed to the plasma for 6 hours, cooled, weighed, stirred and spread out again before being exposed to the plasma another $3-4$ times in 6 hour intervals until ashing was completed. The ashing was assumed to be completed when there was no further weight loss of the ash.

High temperature ashes were prepared from lignite samples at three different temperatures, $723 \mathrm{~K}, 823 \mathrm{~K}$, and $923 \mathrm{~K}$ using a muffle furnace in an atmosphere of air, resulting in thirty ash samples.

\subsubsection{X-Ray Diffractograms (XRD)}

For the high temperature ash samples X-ray diffractograms (XRD) were obtained in a Siemens D500 XRD powder diffractometer using parabolic filtered CuK $\alpha$ radiation at $40 \mathrm{kV}$ and $30 \mathrm{~mA}$. Each sample was scanned at a step size of $2 \theta$ within the scattering range of $0-70^{\circ}$. Jade 6 analytical software was then used for peak identification. For the low temperature ash samples X-ray diffractograms were obtained using a Phillips 
Chapter 3

X'pert diffractometer with copper $\mathrm{K} \alpha$ radiation. The minerals were identified with reference to the JCPDS Powder Diffraction File. Quantitative analyses of the minerals in the LTA were made using SIROQUANT ${ }^{\mathrm{TM}}$ commercial interpretation software based on the Rietveld XRD analysis technique.

\subsubsection{X-ray Fluorescence (XRF)}

X-ray Fluorescence (XRF) analysis was carried out on the high temperature ash samples prepared at $823 \mathrm{~K}$, using a Pananalytical (Philips) Majix fast simultaneous XRF. Matrix corrections were applied using calculated Alpha corrections for this combination of flux, tube and instrument geometry. The samples for XRF were manually pulverised in an agate mortar and pestle and dried in an oven at $378 \mathrm{~K}$ for 6 hours as recommended in ISO-2596. The samples were then fused and cast into $40 \mathrm{~mm}$ diameter glass beads using a 12:22 flux containing 5\% sodium nitrate. A $50 \% \mathrm{NaCl} / 50 \% \mathrm{SiO}_{2}$ synthetic standard was run to check the $\mathrm{Na}$ and $\mathrm{Cl}$ reading and a French iron ore standard was also run during the analysis to check the sulphur calibration.

\subsubsection{Fourier-Transform Infrared (FTIR)}

Fourier-transform infrared (FTIR) analysis was performed using a Perkin Elmer 1760X FTIR spectrometer. Specimens for the analysis were prepared by mixing $4 \mathrm{mg}$ of a lignite or char sample with 400mg potassium bromide (KBR) to produce KBR pellets. The infrared signal was recorded for wave numbers $400-4000 \mathrm{~cm}^{-1} .256$ background and 256 sample scans were co-averaged and ratioed. A mirror velocity of $2.2 \mathrm{kHz}$, and a spectral resolution of 4 wave numbers were used. The FTIR peak spectra were identified by comparing with the literature data $[17,18]$. 


\subsubsection{Nuclear Magnetic Resonance (NMR)}

The lignite structure was studied using a Varian $400 \mathrm{Mhz}$ solid-state 13C Nuclear Magnetic Resonance (NMR) spectrometer with the cross-polarisation technique with high-power proton decoupling and magic angle spinning technique (13C CP-MAS NMR). The 13C CP-MAS NMR was set at a spinning rate of $5 \mathrm{khz}$. The NMR was performed using a Varian $400 \mathrm{MHz}$ NMR spectrometer. To suppress the spinning sidebands, total suppression of spinning sidebands (TOSS) was used.

\subsubsection{Scanning Electron Microscopy (SEM)}

Scanning Electron Microscopy (SEM) was performed on the raw and char lignite samples for morphology studies using a Philips XL30 scanning electron microscope. The samples were dispersed onto a carbon tape which was stuck onto a $10 \mathrm{~mm}$ stub, and coated with carbon.

\subsubsection{Petrography Analysis}

Polished block microscope samples were prepared for the reflectance and maceral counting on the lignite and its char using a Leica DM6000M microscope with a Basler A102F and digital monochrome camera 1392 x 1040 pixels.

\subsection{Pyrolysis Equipments and Techniques}

To study the behaviour of organic and inorganic sulphur during lignite pyrolysis, a fixed bed reactor and a thermogravimetric analyser (TGA) were chosen. Pyrolysis with a fast heating rate was performed in a quartz fixed bed reactor, whereas a slow heating rate 
Chapter 3

was performed in a TGA. The detail of the equipments will be presented in the following sections.

\subsubsection{Fixed Bed Reactor}

A quartz reactor with inside diameter of $28 \mathrm{~mm}$ was used as a fixed bed reactor. The reactor system is shown in Figure 3.1. The fixed bed pyrolysis setting was chosen to simulate the conditions in real processes, for example pyrolysis in a rotary kiln where coal/lignite is continuously fed and experiences long residence time.

Pyrolysis of the lignite samples with particle size ranges $<250 \mu \mathrm{m}$ was carried out in a fast heating mode, the method used was similar to that used by Yip et al. [80]. About 1 gram of the sample was fed into the reactor which had been preheated to the desired temperatures $(673,773,873,973$ and $1073 \mathrm{~K})$ and purged with nitrogen at a constant flow rate of 1 L.min ${ }^{-1}$. The feeding time was about 10 minutes. The chars were accumulated on the quartz frit inside the reactor until the feeding ended. The reactor then was held for 30 minutes before being taken out from the furnace and cooled naturally to room temperature. The chars collected then was weighed and stored in an air tight container before being subjected for sulphur and other analyses. 


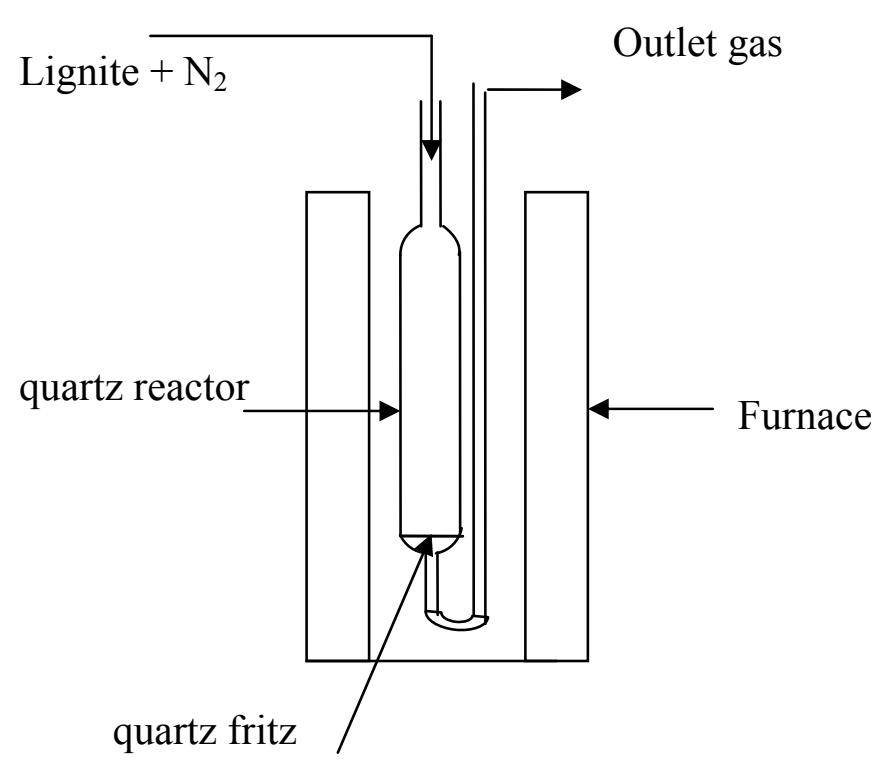

Figure 3.1 A schematic of the fixed bed quartz reactor

\subsubsection{Thermogravimetric Analyser (TGA)}

Pyrolysis was conducted in a simultaneous Differential Scanning Calorimetry (DSC) and Thermogravimetric Analyser (TGA) (TA Instrument Q600) as can be seen in Figure 3.1. TGA pyrolysis was run for slow heating rate. The experiments were carried out at atmospheric pressure. A small mass, $\sim 12 \mathrm{mg}$, of each material, thinly distributed in the silica crucible and particle size $-250 \mu \mathrm{m}$ were found proper to be used in the experiments, in order to eliminate the effects of eventual side reactions and mass and heat transfer limitations. The experiments were replicated at least twice to determine their reproducibility which was found to be very good.

For pyrite transformation during lignite pyrolysis, TGA experiments were conducted for pure pyrite, L1, and L1-pyrite blends under nitrogen atmosphere at $100 \mathrm{ml} . \mathrm{min}^{-1}$ of flow rate from $383 \mathrm{~K}$ to $1273 \mathrm{~K}$ for pyrite and from $383 \mathrm{~K}$ to $1073 \mathrm{~K}$ for other samples. For all the pyrolysis runs, about $12 \mathrm{mg}$ of each sample was used. Thermogravimetric and differential thermogravimetric (DTG) data were recorded during the analysis. Pyrite 
pyrolysis was conducted at heating rates of $10 \mathrm{~K} \cdot \mathrm{min}^{-1}$ and $50 \mathrm{~K} \cdot \mathrm{min}^{-1}$, respectively; while L1 and L1-pyrite blends were pyrolysed at a heating rate of $50 \mathrm{~K} \cdot \mathrm{min}^{-1}$.

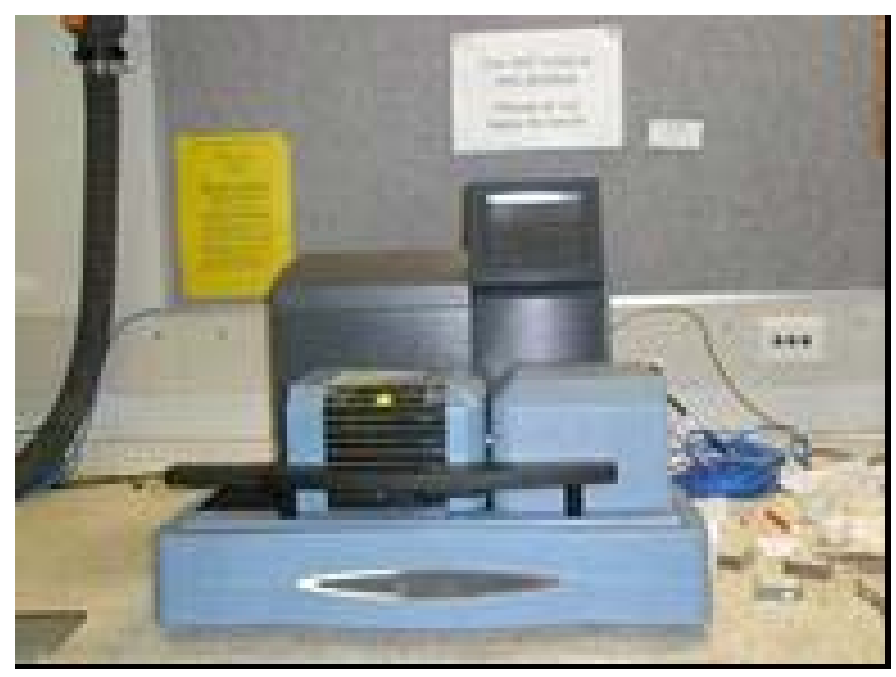

Figure 3.2 TA instrument Q600

The transformation of sulphate during lignite pyrolysis was conducted in a TGA for pure sulphates $\left(\mathrm{CaSO}_{4}, \mathrm{FeSO}_{4}\right.$ and $\left.\mathrm{Fe}_{2}\left(\mathrm{SO}_{4}\right)_{3}\right)$ and acid washed lignite $\mathrm{L} 1$ blends with the sulphates. The transformation of pure sulphates was studied under different gas environments, including nitrogen, air, 50\% nitrogen $+50 \% \mathrm{CO}_{2}$, whereas the blended samples were only studied under nitrogen atmosphere. The pyrolysis was run in a nonisothermal mode with a constant flow rate of $100 \mathrm{ml} \cdot \mathrm{min}^{-1}$ and heating rate of $10 \mathrm{~K} \cdot \mathrm{min}^{-1}$ from room temperature to $1073 \mathrm{~K}$, except for pure calcium sulphate samples which were conducted from room temperature to $1773 \mathrm{~K}$.

\subsection{Sulphur Analyses}

\subsubsection{Sulphur in Solid}

The various lignite samples and their chars were analysed for sulphur forms, namely total sulphur, organic sulphur and inorganic sulphur following the method used by Guo et al. [43] . Figure 3.3 explains the determination of sulphur in a solid sample. The total and organic sulphur was analysed using a carbon sulphur determinator Eltra CS-2000. 
Chapter 3

Organic sulphur was determined by dispersing a sample in a $5 \mathrm{M} \mathrm{HNO}_{3}$ solution, boiled and held for $30 \mathrm{~min}$. When the mixture was cooled, it was washed with double distilled water and filtered. The residue, free of inorganic sulphur, was then dried in the vacuum drier at $333 \mathrm{~K}$ for 48 hours and then analysed for its total sulphur, which is considered to be the organic sulphur as all inorganic sulphur would have been removed during the acid-washing. Inorganic sulphur in a sample was determined by the difference between the total sulphur and organic sulphur.

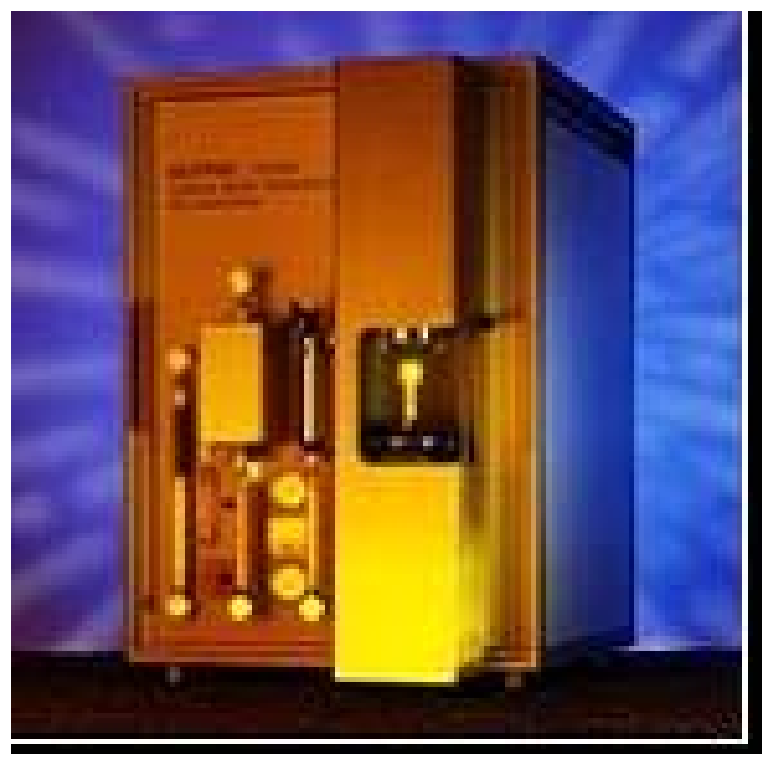

Figure 3.3 Eltra CS 2000

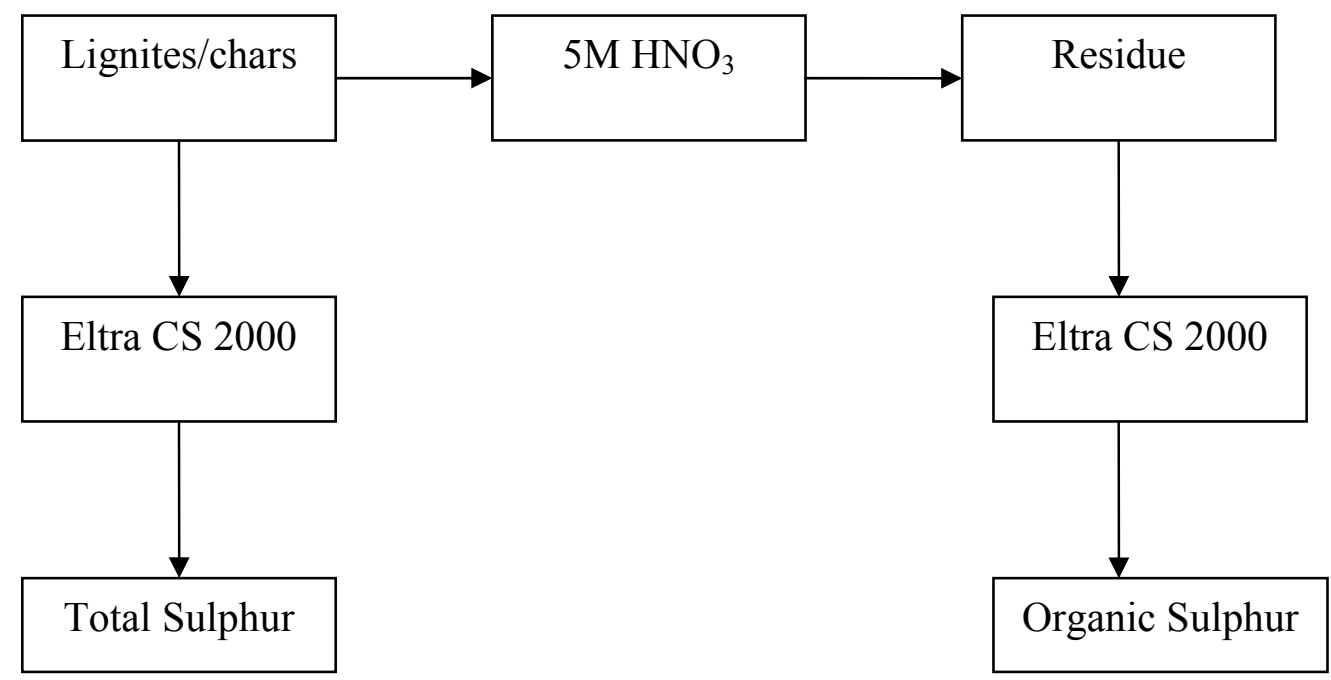

Inorganic Sulphur = Total Sulphur-Organic Sulphur

Figure 3.4 Determination of various forms of sulphur in lignites and chars 
The result of each type of sulphur retained in the char following pyrolysis, i.e. total sulphur, organic sulphur and inorganic sulphur, was then calculated according to:

Sulphur retention $=\frac{X_{S(d b)} \times W_{\text {char }}}{\text { Total } S_{(\text {lig) }} \times W_{\text {lignite }}}$

where $\mathrm{X}_{\mathrm{S}}(\mathrm{db})$ is the weight percentage of a type of sulphur in the sample on dry basis, $\mathrm{W}_{\text {char }}$ is the weight percentage of char yield, Total $\mathrm{S}_{(\mathrm{lig})}$, the weight percentage of total sulphur of the starting lignite on dry basis and $\mathrm{W}_{\text {lignite, }}$ the weight of the starting lignite sample.

Volatile sulphur release during pyrolysis was calculated by the following equation:

Volatile $_{T}=\left(100-\right.$ Total Sulphur retention $\left._{T}\right)$

where Volatile $\mathrm{S}_{\mathrm{T}}$ is the percentage of volatile sulphur released at temperature $\mathrm{T}$ and Total sulphur retention ${ }_{\mathrm{T}}$ is the weight percentage of total sulphur retained in the char at temperature $\mathrm{T}$.

In order to assist in the interpretation of sulphur transformation, chemical structural characterisation of the lignite and its char was performed using FTIR spectroscopy, Solid-state ${ }^{13} \mathrm{C}$ NMR, XRD, and Scanning Electron Microscopy equipped with an energy-dispersive X-ray spectroscopy (SEM-EDS). The instruments and methods used were similar to that described in section 3.2.

\subsubsection{Sulphur in Gas Released during Pyrolysis}

To determine the composition of gaseous products evolved during lignite pyrolysis, experiments were also performed using Netzch PC409 TGA-DSC coupled to a 403C Aeolos Netzch mass spectrometer (TGA-MS). A $150 \mu \mathrm{m}$ deactivated quartz capillary line heated to $473 \mathrm{~K}$ was used to connect the TGA to the MS in order to minimise the 
condensation of high boiling point gases in the transfer line. Figure 3.5 shows the TGAMS used in the experiments.

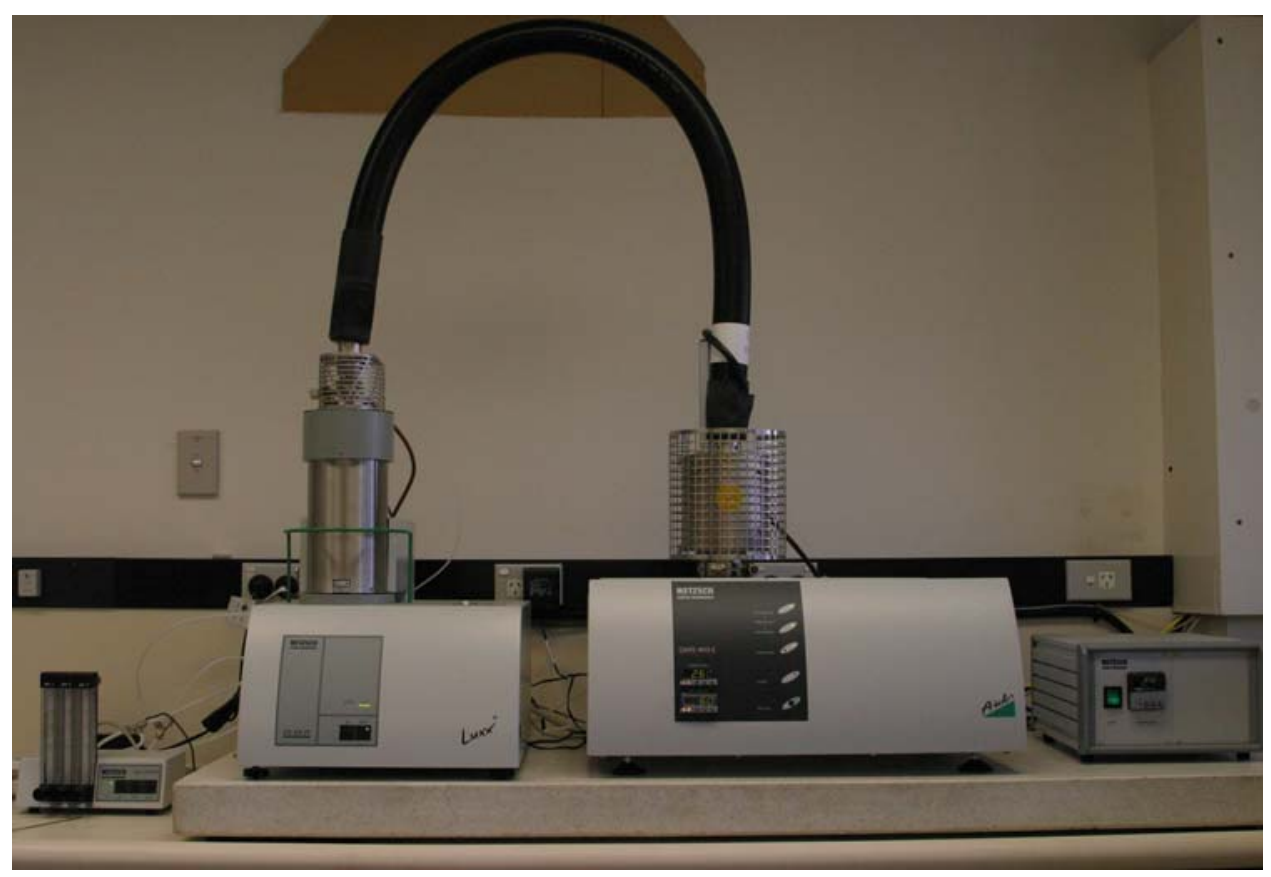

Figure 3.5 Netzch PC409 TGA-DSC coupled to a 403C Aeolos Netzch mass spectrometer (TGA-MS).

A sample of about $30 \mathrm{mg}$ is placed in a ceramic crucible in a TGA and heated from room temperature to $1073 \mathrm{~K}$ at a constant heating rate of $10 \mathrm{~K} \cdot \mathrm{min}^{-1}$ in argon with a constant flow rate of $100 \mathrm{mLmin}^{-1}$. The MS system automatically samples gases evolved from TGA system, analyse them and save the mass spectra. The interested mass spectra range for the experiments is $1-100$ atomic mass unit $(\mathrm{amu})$ or $\mathrm{m} / \mathrm{z}=1-100$.

\subsection{Summary}

A number of complimentary sample preparation, characterisation and experimental techniques were employed for the investigation of sulphur transformation during lignite pyrolysis. The lignite preparations and analytical methods enabled to study the transformation of pyrite, sulphate and organic sulphur as well as the effect of mineral 
Chapter 3

matters in the sulphur transformation during pyrolysis of lignite which contains high oxygenated structures as discussed in details in chapter 4. Lignite pyrolysis experiments in a fixed bed quartz reactor were conducted to study the sulphur transformation in a fast heating rate mode, while the TGA experiments were conducted in a slow heating rate mode. TGA coupled to MS enabled to study the evolution of gaseous sulphur during lignite pyrolysis online. 


\section{CHAPTER 4}

\section{Lignite Characterisation}

\subsection{Introduction}

In this chapter, the characteristics of 11 lignite samples from Western Australia, namely L1-L11, respectively, are presented. Approximately $10 \mathrm{~kg}$ of each sample was mixed before subjected for characterisation. A combination of techniques was used to characterise the lignite samples and their various char products. X-ray diffraction (XRD) and X-ray Fluorescence (XRF) were used to identify the minerals present in lignite ash, Fourier transform infra red (FTIR) and solid state ${ }^{13} \mathrm{C}$ nuclear magnetic resonance (NMR) were used to characterise the structure of the lignite and the maceral was identified by petrographic analysis. Based on the lignite characterisation results, some lignites were chosen for further research on the sulphur transformation.

\subsection{Lignite properties}

The properties of lignites including proximate, ultimate and sulphur forms analyses as well as calorific values of raw samples are presented and discussed in this section. Proximate analysis was carried out to determine moisture, ash, volatile matter and fixed carbon contents in coal. Ultimate analysis was carried out to determine $\mathrm{C}, \mathrm{H}, \mathrm{N}, \mathrm{S}, \mathrm{O}$ and mineral and inorganic contents in the samples. Proximate and ultimate analysis, sulphur forms and calorific values of the samples are shown in Tables 4.1-4.4, respectively. 
Chapter 4

The moisture content as presented in Table 4.1 is the 'as received' value. The lignites analysed are very wet samples having moisture contents ranging from 55.6 to $64.9 \%$. Since the lignites are very wet samples, drying is the important step to be done before utilising these lignites.

In comparison to world standards of commercially exploited lignite, all the lignite samples have very high ash contents ranging from 20.7 to $32.7 \%$ dry basis (db), except for lignite L1 which only contains $3.1 \% \mathrm{db}$ ash yield. For example the ash contents of the lignites of Latrobe Valley range from 0.2 to 1.5 per cent [81]. The high ash content of the lignites imply that the lignites have high mineral and inorganic matter content. The volatiles of the lignites are also high, ranging from 38.6 to $52.6 \% \mathrm{db}$.

Table 4.1. Proximate analysis

\begin{tabular}{c|cccc}
\hline Samples & Moisture (\% ar ) & Ash yield (\%db) & VM (\%db) & FC (\%db) \\
& & & & \\
\hline L1 & 64.9 & 3.1 & 52.6 & 44.3 \\
L2 & 55.9 & 27.1 & 41.3 & 31.6 \\
L3 & 59.6 & 27.7 & 40.9 & 31.4 \\
L4 & 53.8 & 24.0 & 43.3 & 32.7 \\
L5 & 57.8 & 31.6 & 43.8 & 24.6 \\
L6 & 55.6 & 28.9 & 41.1 & 30 \\
L7 & 62.7 & 28.0 & 42.5 & 29.5 \\
L8 & 57.4 & 29.7 & 39.8 & 30.5 \\
L9 & 56.2 & 32.7 & 38.6 & 28.7 \\
L10 & 54.8 & 20.7 & 43.9 & 35.4 \\
L11 & 55.8 & 22.0 & 41.1 & 36.9 \\
\hline ar = as received & & &
\end{tabular}

The fixed carbon (FC) contents of all the samples vary from 24.6 to $44.3 \% \mathrm{db}$. With the exception of sample L1, the low FC contents of the lignites are due to the high volatile matter and the high ash contents for all the samples. 
Table 4.2. Ultimate analysis (\% dry basis)

\begin{tabular}{c|lrrrc}
\hline Samples & \multicolumn{1}{l}{ C } & \multicolumn{1}{l}{$N$} & S (org) & O (by diff) \\
\hline L1 & 66.4 & 5.1 & 0.48 & 0.21 & 25.5 \\
L2 & 45.9 & 3.8 & 0.29 & 1.74 & 24.4 \\
L3 & 48.5 & 3.9 & 0.28 & 1.56 & 23.6 \\
L4 & 44.1 & 4.1 & 0.25 & 0.02 & 27.5 \\
L5 & 43.2 & 4.2 & 0.25 & 1.33 & 19.4 \\
L6 & 46.7 & 3.8 & 0.31 & 1.92 & 23.1 \\
L7 & 45.8 & 4.2 & 0.30 & 1.67 & 20.0 \\
L8 & 46.6 & 3.8 & 0.29 & 1.61 & 20.9 \\
L9 & 41.5 & 3.5 & 0.34 & 1.27 & 20.7 \\
L10 & 46.4 & 3.9 & 0.32 & 1.22 & 27.5 \\
L11 & 45.0 & 3.9 & 0.30 & 0.98 & 27.8 \\
\hline
\end{tabular}

Table 4.3. Sulphur forms (\% dry basis)

\begin{tabular}{c|cccc}
\hline $\begin{array}{l}\text { Sample } \\
\text { Description }\end{array}$ & Total S & $\begin{array}{l}\text { Sulphate } \\
\text { S }\end{array}$ & Pyritic S & Organic S \\
\hline L1 & 0.24 & 0.03 & - & 0.21 \\
L2 & 4.62 & 1.27 & 1.61 & 1.74 \\
L3 & 2.18 & 0.56 & 0.06 & 1.56 \\
L4 & 1.37 & 0.96 & 0.40 & 0.02 \\
L5 & 1.88 & 0.54 & 0.01 & 1.33 \\
L6 & 3.02 & 0.90 & 0.20 & 1.92 \\
L7 & 2.35 & 0.63 & 0.05 & 1.67 \\
L8 & 2.90 & 0.62 & 0.67 & 1.61 \\
L9 & 2.56 & 0.90 & 0.38 & 1.27 \\
L10 & 2.81 & 1.27 & 0.32 & 1.22 \\
L11 & 1.86 & 0.77 & 0.11 & 0.98 \\
\hline
\end{tabular}

From Table 4.3 it can be seen that the lignites have a high total sulphur content ranging from 1.37 to $4.62 \% \mathrm{db}$, except for lignite $\mathrm{L} 1$ which contains only $0.24 \% \mathrm{db}$ total sulphur. L3 and L5-L11 have a high percentage of organic sulphurs and low pyritic sulphur content, whereas L4 has low organic sulphur and high sulphate sulphur. Sulphate sulphurs, pyritic sulphurs and organic sulphurs are present significantly in L2, the lignite with the highest total sulphur. L1 has the lowest sulphur content with no pyrite sulphur detected on the sample. 
Chapter 4

Calorific values of the lignites were measured for gross dry calorific values, gross wet calorific values and net wet calorific values (Table 4.4). The gross wet calorific values of the lignites are ranging from 17.8 to $26.6 \mathrm{MJ} \cdot \mathrm{kg}^{-1}$. These values are categorised as low calorific values, because the standard fuel energy of coal is about $32 \mathrm{MJ}^{\mathrm{kg}}{ }^{-1}[82]$

From the results of the analysis it can be concluded that the lignites are of low quality due to their high moisture, ash, volatiles, and sulphur content and low fixed carbon content and calorific value.

Table 4.4. Calorific value

\begin{tabular}{|c|c|c|c|}
\hline Samples & $\begin{array}{l}\text { Gross Dry } \\
\text { Calorific } \\
\text { Value } \\
\left(M J . \mathrm{kg}^{-1}\right)\end{array}$ & $\begin{array}{l}\text { Gross Wet } \\
\text { Calorific } \\
\text { Value } \\
\left(M^{\prime} . \mathrm{kg}^{-1}\right)\end{array}$ & $\begin{array}{l}\text { Net Wet } \\
\text { Calorific } \\
\text { Value } \\
\left(M J . \mathrm{kg}^{-1}\right)\end{array}$ \\
\hline L1 & 26.6 & 9.3 & 7.5 \\
\hline L2 & 18.9 & 8.3 & 6.7 \\
\hline L3 & 20.0 & 8.1 & 6.4 \\
\hline L4 & 19.4 & 9.0 & 7.3 \\
\hline L5 & 19.1 & 8.1 & 6.4 \\
\hline L6 & 19.3 & 8.6 & 6.9 \\
\hline L7 & 19.4 & 7.2 & 5.5 \\
\hline L8 & 19.0 & 8.1 & 6.4 \\
\hline L9 & 17.8 & 7.8 & 6.2 \\
\hline L10 & 19.9 & 9.0 & 7.4 \\
\hline L11 & 19.1 & 8.5 & 6.8 \\
\hline
\end{tabular}




\subsection{Mineralogy}

\subsubsection{Low temperature ashing (LTA)}

The mineralogy of the low-temperature ash (LTA) for four samples (L2, L3, L6 and L8) is presented in Figure 4.1 and Table 4.5. The percentages of halite in the samples are quite high except for sample L8. The sulphate minerals (loeweite, hexahydrite and bassanite) present in the samples are also found to be very high (about $42 \%$ to $58 \%$ ), which is believed to be an artefact of the ashing process[20].

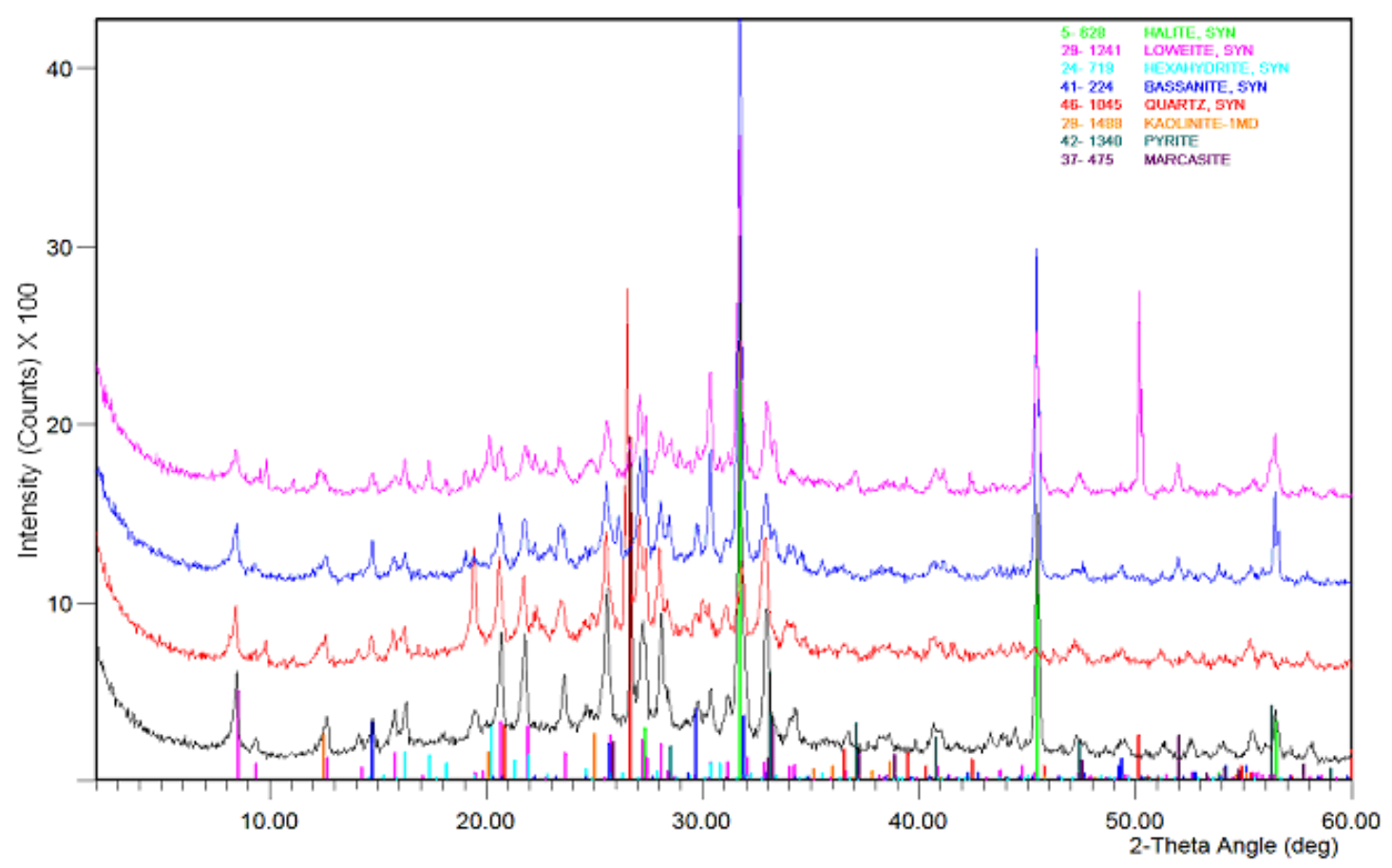

Figure 4.1. X-ray diffractograms of LTA samples L2, L3, L5, L7

Table 4.5. Mineralogy of LTA samples (A2, B2, C1 and D) using powder XRD and Siroquant ${ }^{\mathrm{TM}}$

\begin{tabular}{|c|c|c|c|c|c|c|c|c|}
\hline Samples & $\begin{array}{l}\text { Halite } \\
\mathrm{NaCl} \\
\text { (\%) }\end{array}$ & $\begin{array}{l}\text { Loeweite } \\
\mathrm{Na}_{12} \mathrm{Mg}_{7}\left(\mathrm{SO}_{4}\right)_{13} .15 \mathrm{H}_{2} \mathrm{O} \\
(\%)\end{array}$ & $\begin{array}{l}\text { Hexahydrite } \\
\mathrm{MgSO}_{4} 6 \mathrm{H}_{2} \mathrm{O} \\
(\%)\end{array}$ & $\begin{array}{l}\text { Bassanite } \\
\mathrm{CaSO}_{4.1 / 2 \mathrm{H}_{2} \mathrm{O}} \\
(\%)\end{array}$ & $\begin{array}{l}\text { Quartz } \\
\mathrm{SiO}_{2} \\
(\%)\end{array}$ & $\begin{array}{l}\text { Kaolinite } \\
\mathrm{Al}_{2} \mathrm{Si}_{2} \mathrm{O}_{5}(\mathrm{OH})_{2} \\
(\%)\end{array}$ & $\begin{array}{l}\text { Pyrite } \\
\text { FeS }_{2} \\
(\%)\end{array}$ & $\begin{array}{l}\text { Marcasite } \\
\mathrm{FeS}_{2}(\%)\end{array}$ \\
\hline L2 & 18.2 & 26.4 & 19.6 & 7.5 & 2.1 & 14.8 & 8.2 & 3.3 \\
\hline L3 & 17.8 & 38.4 & 8.3 & 5.9 & 10.5 & 12.5 & 6.6 & 0 \\
\hline L8 & 1.9 & 36.7 & 15 & 6 & 16.4 & 13.6 & 10.3 & 0 \\
\hline L6 & 32.2 & 23.8 & 7.4 & 10.9 & 1 & 13.8 & 3.9 & 6.9 \\
\hline
\end{tabular}




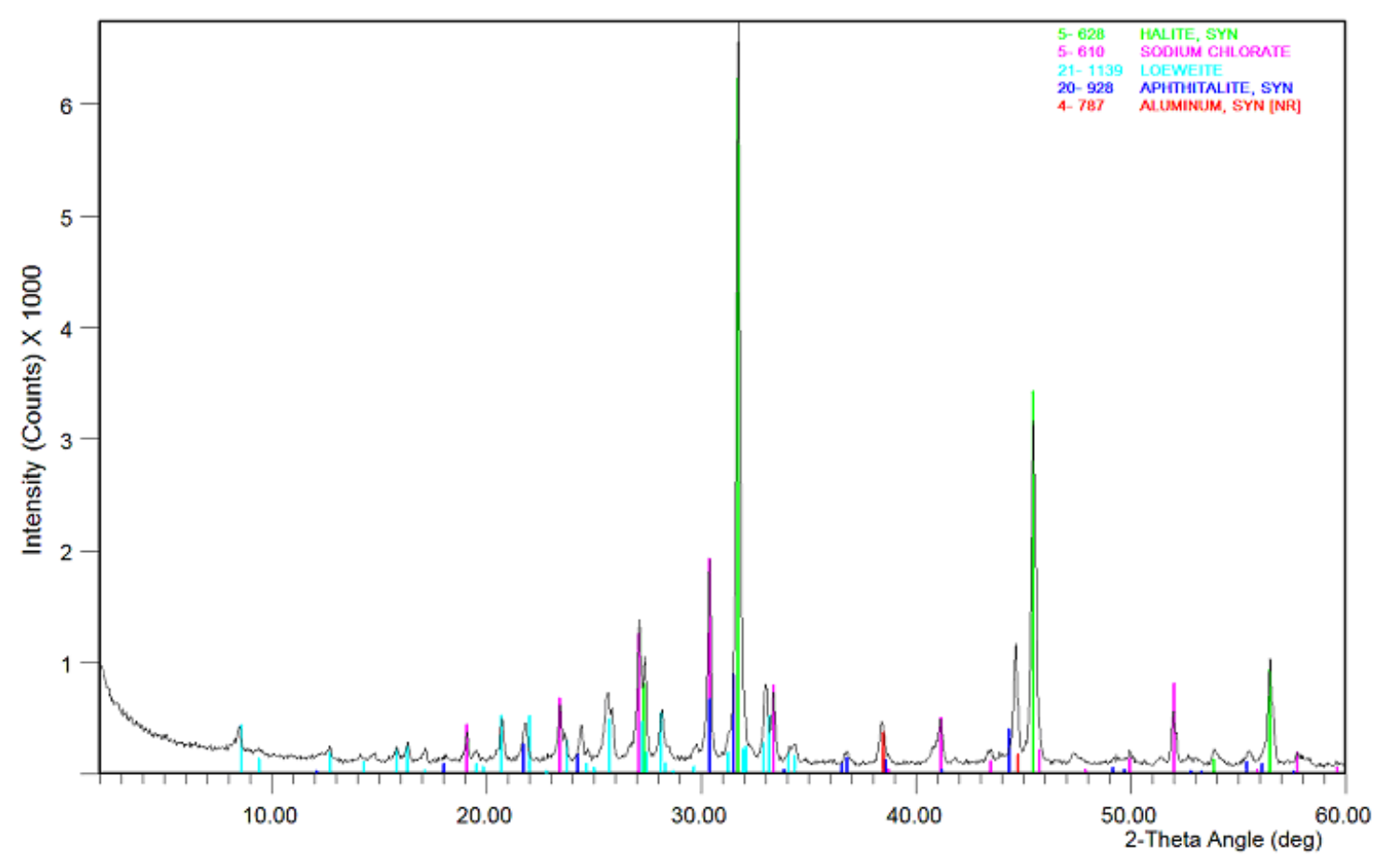

Figure 4.2. X-ray diffractogram of LTA sample L4

The x-ray diffractograms for samples L4 and L11, with the peaks attributed to each mineral, is shown in Figures 4.2 and 4.3, respectively. The minerals identified in sample L11 was sodium chlorate hydrate $\left(\mathrm{NaClO}_{4} \cdot \mathrm{H}_{2} \mathrm{O}\right)$ and vanthoffite $\left(\mathrm{Na}_{6} \mathrm{Mg}\left(\mathrm{SO}_{4}\right)_{4}\right)$, while for sample L4 the minerals halite $(\mathrm{NaCl})$, sodium chlorate $\left(\mathrm{NaClO}_{4} \cdot \mathrm{H}_{2} \mathrm{O}\right)$, loweite $\left(\mathrm{Na}_{12} \mathrm{Mg}_{7}\left(\mathrm{SO}_{4}\right)_{13} .15 \mathrm{H}_{2} \mathrm{O}\right)$ and aphthitalite $\left(\mathrm{K}_{2} \mathrm{Na}\left(\mathrm{SO}_{4}\right)_{2}\right)$ was identified. The minerals in sample L4 and L11 that were identified were sodium containing minerals. Quantitative analysis of the phases present using Siroquant was not possible, as several phases, particularly the sodium chlorate and the aphthitalite are not included in the current database. Although the organic sulphur in L4 was not as high as other samples, sulphates were still identified from LTA for L4.

It shows from the above explanation that the sulphates identified from LTA of the lignite samples are quite high. Interaction between non-mineral inorganics and organic sulphurs may lead to the formation of sulphate sulphurs during LTA process [20, 26, 
83]. Therefore, the sulphates identified may represent artefacts of the low temperature ashing, not constituents really occurring in the mineral forms of the coal.

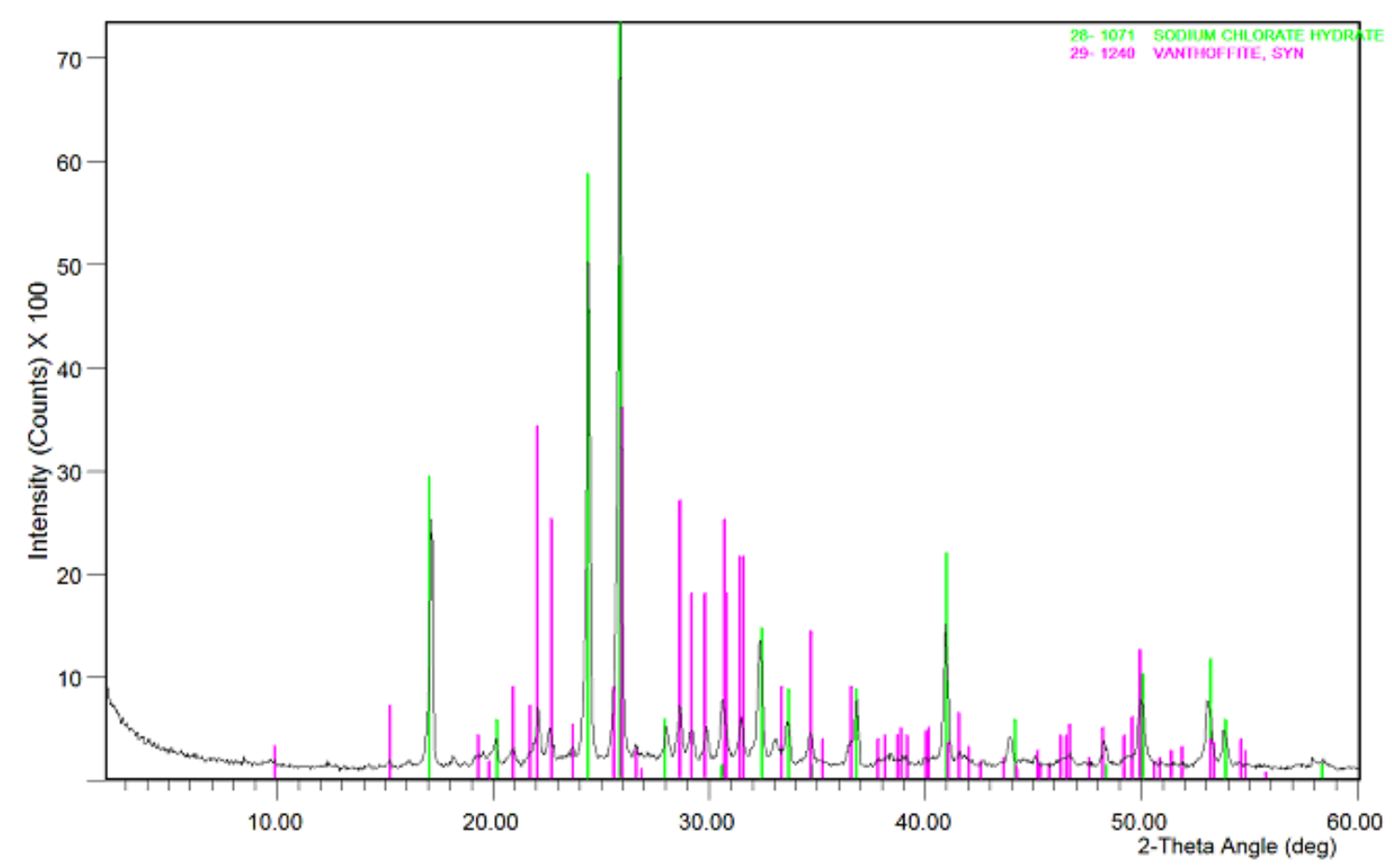

Figure 4.3. X-ray diffractogram of LTA sample L11

From section the previous paragraphs, the lignites are identified to contain very high inorganic matter, especially $\mathrm{Cl}$ and $\mathrm{Na}$, except for lignite L1. From sulphur forms analysis presented in Table 4.3, it can be seen that the organic sulphur are found to be significant in all samples analysed for their LTA, except for sample L4, with moderate content of sulphate sulphur. Therefore, the high sulphates found in LTA of the lignites are not sulphates really exist in the lignite samples. From this finding, it can be confirmed that identification of minerals in raw lignite using LTA is not suitable.

\subsubsection{High temperature ashing}

To further study the mineralogy of the ash from raw lignite samples, high temperature ashing was performed at three different temperatures 723, 823 and $923 \mathrm{~K}$. The ash yield 
Chapter 4

prepared in the three different temperatures is shown in Table 4.6. It can be seen that all samples contain quite high ash, except sample L1 which only has ash content between $3.10-3.35 \%$ in three different temperatures. Almost all samples have a slight decrease in ash yield with increase in ashing temperature, except for sample L6 and L7 which experience significant decrease in ash yield with increasing temperature.

Table 4.6. Ash yield of samples prepared at different temperatures

\begin{tabular}{l|ccc}
\hline Samples & $\begin{array}{c}723 K \\
(\%)\end{array}$ & $\begin{array}{c}823 K \\
(\%)\end{array}$ & $\begin{array}{c}923 K \\
(\%)\end{array}$ \\
\hline L1 & 3.35 & 3.24 & 3.10 \\
L2 & 13.69 & 10.34 & 12.74 \\
L3 & 10.78 & 9.63 & 9.62 \\
L4 & 15.95 & 14.43 & 14.32 \\
L5 & 14.17 & 10.72 & 11.51 \\
L6 & 13.96 & 12.85 & 12.56 \\
L7 & 14.91 & 13.19 & 9.81 \\
L8 & 16.06 & 12.07 & 11.69 \\
L9 & 14.23 & 12.74 & 12.15 \\
L10 & 13.12 & 12.48 & 12.18 \\
L11 & 15.02 & 14.06 & 13.60 \\
\hline
\end{tabular}

The mineralogy of ash samples was then analysed by XRD and the ash prepared at 923 K was analysed by XRF. Figure 4.4 shows the $\mathrm{X}$-ray diffractogram for sample L2. The x-ray diffractograms for the other lignite ashed samples were quite similar and is therefore shown in the Appendix. From the results it can be seen that the lignite predominantly contain halite $(\mathrm{NaCl})$ at all temperatures with the amount of magnesioferrite $\left(\mathrm{MgFe}_{2} \mathrm{O}_{4}\right)$ increasing at higher temperatures. However, for other lignite ashes prepared at different temperatures only the halite peaks were identified in the XRD spectra. In comparison to the LTA, LTA samples are dominated by sulphate (which could be due to artefacts of the ashing process) whereas the high temperature ashes are dominated by halite. 


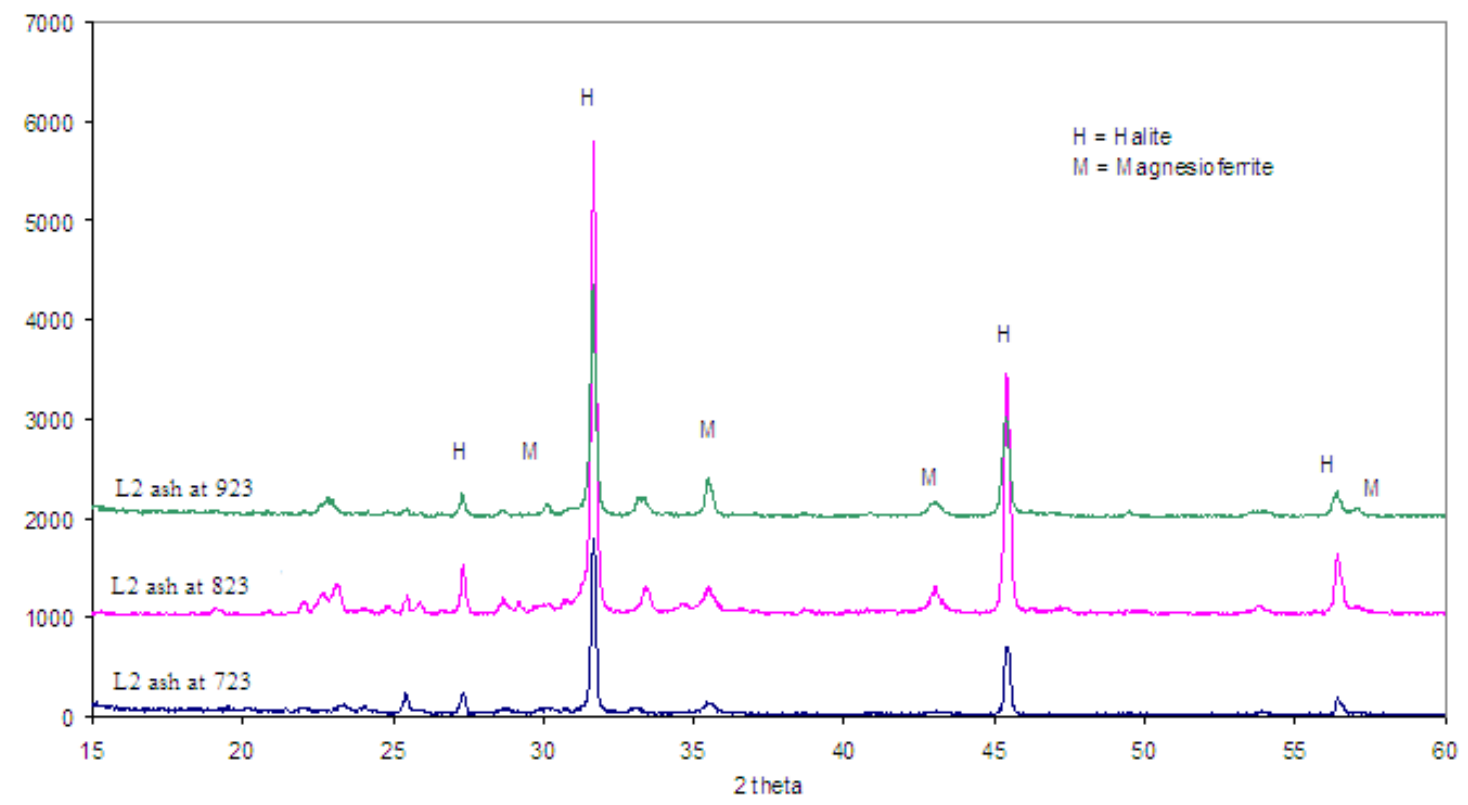

Figure 4.4. X-ray diffractogram of sample L2 ash prepared at three different temperatures 723,823 and $923 \mathrm{~K}$

Table 4.7 shows the XRF results of the ash prepared at $923 \mathrm{~K}$. It can be observed that the lignites predominantly contain sodium followed by sulphur. $\mathrm{Fe}, \mathrm{Si}, \mathrm{Al}$ and $\mathrm{Mg}$ are also present in significant amounts for some of the lignites.

To ensure that the minerals identified from HTA at $923 \mathrm{~K}$ represents the minerals really occur in the sample, some of the lignite samples were analysed for their mineral and inorganic values based on a combination of AS1038.14.1, AS2434.9, AS2434.6.1-part-8 and AS1038.8.2. The results of the analysis are presented in Table 4.8. The results of the mineralogy of HTA at $923 \mathrm{~K}$ are quite representing the minerals content of the lignites obtained from combinations of Australia Standard methods. Sodium is found to be extremely high in all lignites. 
Table 4.7. XRF data for lignite samples ashed at $923 \mathrm{~K}$

\begin{tabular}{|c|c|c|c|c|c|c|c|c|c|}
\hline Samples & $\begin{array}{l}\mathrm{SiO}_{2} \\
(\%)\end{array}$ & $\begin{array}{l}\mathrm{Al}_{2} \mathrm{O}_{3} \\
(\%)\end{array}$ & $\begin{array}{l}\mathrm{CaO} \\
(\%)\end{array}$ & $\begin{array}{l}\mathrm{Fe}_{2} \mathrm{O}_{3} \\
(\%)\end{array}$ & $\begin{array}{l}\mathrm{K}_{2} \mathrm{O} \\
(\%) \\
\end{array}$ & $\begin{array}{l}\text { MgO } \\
(\%)\end{array}$ & $\begin{array}{l}\mathrm{Na}_{2} \mathrm{O} \\
(\%)\end{array}$ & $\begin{array}{l}\mathrm{SO}_{3} \\
(\%)\end{array}$ & $\begin{array}{l}\mathrm{TiO}_{2} \\
\text { (\%) }\end{array}$ \\
\hline $\begin{array}{l}\text { L1 } \\
\end{array}$ & 0.91 & $<0.1$ & 0.07 & $<0.1$ & 1.28 & 0.14 & 8.32 & 2.56 & 2.62 \\
\hline L2 & 5.6 & 3.42 & 1.90 & 11.40 & 1.20 & 8.25 & 25.30 & 20.40 & 0.11 \\
\hline L3 & 0.99 & 0.39 & 3.19 & 0.40 & 0.90 & 13.30 & 40.30 & 13.20 & 0.06 \\
\hline L4 & 1.80 & 0.60 & 1.74 & 3.34 & 0.90 & 8.12 & 43.00 & 12.20 & 0.07 \\
\hline L5 & 14.60 & 11.70 & 1.80 & 0.46 & 1.06 & 8.09 & 33.10 & 8.06 & 0.97 \\
\hline L6 & 3.75 & 3.07 & 1.74 & 5.55 & 0.72 & 7.27 & 38.40 & 17.70 & 0.04 \\
\hline L7 & 11.00 & 8.36 & 2.71 & 0.20 & 1.14 & 10.80 & 33.60 & 9.77 & 0.21 \\
\hline L8 & 18.70 & 2.80 & 3.29 & 3.46 & 1.25 & 11.40 & 27.80 & 15.90 & 0.12 \\
\hline L9 & 7.18 & 6.96 & 1.60 & 2.24 & 0.69 & 6.88 & 38.10 & 12.90 & 0.36 \\
\hline L10 & 2.15 & 0.32 & 2.29 & 16.00 & 1.17 & 9.44 & 32.90 & 22.60 & 0.04 \\
\hline L11 & 1.11 & 0.11 & 2.09 & 9.88 & 0.57 & 7.88 & 38.80 & 16.90 & 0.05 \\
\hline
\end{tabular}

Table 4.8. Mineral and Inorganic Forms

\begin{tabular}{l|lllllllll}
\hline Samples & $\begin{array}{l}\mathrm{SiO}_{2} \\
\% d b\end{array}$ & $\begin{array}{l}\mathrm{Al}_{2} \mathrm{O}_{3} \\
\% d b\end{array}$ & $\begin{array}{l}\mathrm{CaO} \\
\% d b\end{array}$ & $\begin{array}{l}\mathrm{Fe}_{2} \mathrm{O}_{3} \\
\% d b\end{array}$ & $\begin{array}{l}\mathrm{K}_{2} \mathrm{O} \\
\% d b\end{array}$ & $\begin{array}{l}\mathrm{MgO} \\
\% d b\end{array}$ & $\begin{array}{l}\mathrm{Na}_{2} \mathrm{O} \\
\% d b\end{array}$ & $\begin{array}{l}\mathrm{SO}_{3} \\
\% d b\end{array}$ & $\begin{array}{l}\mathrm{TiO}_{2} \\
\% d b\end{array}$ \\
\hline $\mathrm{L} 4$ & 2.5 & 0.6 & 2.0 & 5.7 & 0.26 & 8.8 & 33.5 & 11.7 & 0.09 \\
$\mathrm{~L} 5$ & 14.6 & 13.0 & 1.7 & 0.5 & 0.94 & 7.6 & 29.7 & 10.0 & 0.66 \\
$\mathrm{~L} 8$ & 12.3 & 10.1 & 2.0 & 0.9 & 0.77 & 9.1 & 30.8 & 14.1 & 0.37 \\
$\mathrm{~L} 9$ & 7.9 & 8.0 & 1.6 & 4.4 & 0.50 & 5.7 & 34.5 & 16.7 & 0.25 \\
$\mathrm{~L} 10$ & 1.8 & 0.6 & 2.3 & 9.3 & 0.36 & 11.6 & 32.3 & 12.7 & 0.03 \\
$\mathrm{~L} 11$ & 1.1 & 0.5 & 2.3 & 2.0 & 0.23 & 11.8 & 34.4 & 9.9 & 0.02 \\
\hline
\end{tabular}

\section{4 $\mathrm{Cl}$ and Na contents}

From XRD spectra of the high ash samples, halite is found to be the most dominant peak. The percentage of $\mathrm{Na}_{2} \mathrm{O}$ of high ash samples as shown in table 4.7 and 4.8 and the sodium containing compounds identified from LTA samples (Table 4.5 and Figure 4.14.3) are also very significant. Therefore, it is very important to study the form of sodium and chlorine in the lignite samples.

The sodium and chlorine contents were obtained using Ion Chromatography (IC) analysis. The analysis was conducted for water soluble chlorine and sodium and water insoluble chlorine. The results of the analyses are shown in Table 4.9. The water soluble 
sodium contents range from 2.01 to 7.63 per cent, except for L1 which only contains $0.06 \% \mathrm{Na}$. Water soluble chloride contents range between 3.85 to 11.92 per cent, except for $\mathrm{L} 1$ with only $0.08 \% \mathrm{Cl}$. The world average $\mathrm{Cl}$ contents in coal (coal Clarke of $\mathrm{Cl}$ ) for lignite is $120 \pm 20 \mathrm{ppm}$ [84], therefore with the exception of lignite L1, the lignites are classified as having an extremely high chlorine content.

Table 4.9. Water soluble $\mathrm{Na}, \mathrm{Cl}$, and $\mathrm{NaCl}$

\begin{tabular}{l|lll}
\hline Samples & $\begin{array}{l}\mathrm{Na} \\
(\%)\end{array}$ & $\begin{array}{l}\mathrm{Cl} \\
(\%)\end{array}$ & $\begin{array}{l}\mathrm{NaCl} \\
(\%)\end{array}$ \\
\hline L1 & 0.06 & 0.08 & 0.13 \\
L2 & 5.58 & 8.10 & 13.36 \\
L3 & 6.23 & 10.0 & 15.78 \\
L4 & 3.50 & 6.43 & 7.61 \\
L5 & 2.77 & 5.31 & 7.02 \\
L6 & 7.63 & 11.92 & 19.39 \\
L7 & 2.01 & 3.85 & 6.34 \\
L8 & 5.34 & 7.37 & 12.15 \\
L9 & 3.13 & 5.94 & 7.96 \\
L10 & 2.60 & 4.79 & 6.61 \\
L11 & 3.29 & 5.60 & 9.22
\end{tabular}

To confirm whether all the sodium and chlorine are water soluble, total chlorine and sodium analyses were performed by digesting four samples, namely L2, L3, L6 and L8, respectively, in dilute nitric acid. However, no $\mathrm{Cl}$ peak was detected by IC because all the chlorine had already gone during the digestion. Total chlorine analysis was then performed by dissolving the raw dried samples in $0.5 \mathrm{M} \mathrm{NaHCO}_{3}$, which is a method used to analyse total chlorine in soil samples [85]. The $\mathrm{Cl}$ content in the raw lignite samples was quite similar to the water soluble $\mathrm{Cl}$ presented in Table 4.9 so the results were not reported. Almost all the chlorine in the lignite was dissolved during water soluble analysis, since the $\mathrm{Cl}$ in the lignite samples is in the $\mathrm{NaCl}$ form which will dissolve in the water. To support this finding, about $1 \mathrm{~g}$ of water-washed samples was dissolved in $40 \mathrm{~mL} 0.5 \mathrm{M} \mathrm{NaHCO}$. The results are presented in Table 4.10. It can be 
seen that $\mathrm{Cl}$ remained in the water washed sample is very low compared to the $\mathrm{Cl}$ content of the lignites. Thus, it can be concluded that almost all chlorine in the lignites is present as water soluble chlorine.

Table 4.10. $\mathrm{Cl}$ in water washed samples

\begin{tabular}{l|l}
\hline Samples & $\begin{array}{l}\mathrm{Cl} \\
(\mathrm{ppm})\end{array}$ \\
\hline L1 & 5 \\
L2 & 197 \\
L3 & 156 \\
L5 & 519 \\
L7 & 133 \\
\hline
\end{tabular}

$\mathrm{Cl}$ and $\mathrm{Na}$ analysis using IC has shown that $\mathrm{Cl}$ and $\mathrm{Na}$ presents in the lignites is in the form of water soluble $\mathrm{Cl}$ and $\mathrm{Na}$, most of Chlorine and sodium presents in the form of $\mathrm{NaCl}$. Therefore, the XRD spectra of the HTA obtained from three different temperatures show dominant peaks of halite. It is, therefore, very important to study the effect of this inorganic on the sulphur transformation during lignite pyrolysis.

\subsection{Structural and Chemical characterisation}

In order to understand the structural and chemical characteristic of the lignite samples, raw lignite will be compared to the lignite char prepared from lignite pyrolysis in a fixed bed reactor at $823 \mathrm{~K}$ under a nitrogen atmosphere. The raw and char samples are characterised by FTIR and Solid state ${ }^{13} \mathrm{C}$ NMR.

FTIR has been widely used to determine functional groups in coal and its processing products $[18,86,87]$. This technique is considered as a powerful technique in coal characterisation since FTIR is able to detect carbohydrogenated structures (aliphatic and 
Chapter 4

aromatic structures), heteroaromatic functional groups (mainly as oxygenated structures) and also minerals present in the coal [18].

The infrared spectra of L1 and L2 and their chars are shown in Figure 4.5 and 4.6, respectively. The identification of the most prominent bands of the FTIR spectra is shown in Table 4.11. The bands were identified by comparison to literature[17, 18, 87 , 88]. The infrared spectrum for the other lignites and their chars were quite similar and is therefore shown in the Appendix.

The spectra of two raw lignites, L1 and L2, are quite similar, except for the peaks identified at $400-600 \mathrm{~cm}^{-1}$ and $3600-4000 \mathrm{~cm}^{-1}$ region. Bands in the region 3600-4000 and $400-600 \mathrm{~cm}^{-1}$ represent the clay minerals such as pyrite, kaolinte, illite and silicate minerals present in the lignite $[88,89]$. L1 is pyrite-free lignite with very low minerals content, while L2 and other samples have a considerable amount of pyrite and minerals. It can be seen from Figure 4.5 that the mineral bands of L1 is not as intense as the mineral bands of L2 shown in Figure 4.6. From the lignite spectra it can be seen that there is a broad hydroxyl group $(\mathrm{O}-\mathrm{H})$ band with a peak at about $3400 \mathrm{~cm}^{-1}$ for the raw lignite due to the water content of the lignite. For the raw lignite there are two dominant peaks in the aliphatic $\mathrm{CH}$ stretching region $\left(2800-3000 \mathrm{~cm}^{-1}\right)$ at about 2920 and 2850 $\mathrm{cm}^{-1}$, attributed to asymmetric and symmetric $-\mathrm{CH}_{2}$ stretching, respectively. A great abundance of $\mathrm{C}=\mathrm{O}$ and $\mathrm{C}-\mathrm{O}-\mathrm{R}$ structures is observed, as revealed by the intensity of the peaks in the $1800-1000 \mathrm{~cm}^{-1}$ region. The oxygenated structures $\mathrm{C}=\mathrm{O}$ and $\mathrm{C}-\mathrm{O}$ are represented by peaks at around 1602 and $1137 \mathrm{~cm}^{-1}$, respectively. The $\mathrm{C}=\mathrm{C}$ bands which should be placed between $\mathrm{C}=\mathrm{O}$ and $\mathrm{C}-\mathrm{O}$ bands is not clearly identified. This is due to the lignite contains high oxygenated structures and these bands almost masked the $\mathrm{C}=\mathrm{C}$ structures (Yaman, 2000). The aromatic structure in low intensity is identified 
at $700-900 \mathrm{~cm}^{-1}$ region, the bands could be related to aromatic out of plane $\mathrm{C}-\mathrm{H}$ deformation [18].

Table 4.11. Band assignments of the most prominent peaks in the FTIR spectra

\begin{tabular}{l|l}
\hline Bands, $\mathrm{cm}^{-1}$ & Assignments \\
\hline $3600-4000$ & Minerals \\
3400 & -OH stretching \\
$2800-3000$ & Aliphatic CH stretching \\
$1000-1800$ & Oxygenated structure \\
$700-900$ & Aromatic CH bands \\
$400-600$ & Minerals \\
\hline
\end{tabular}

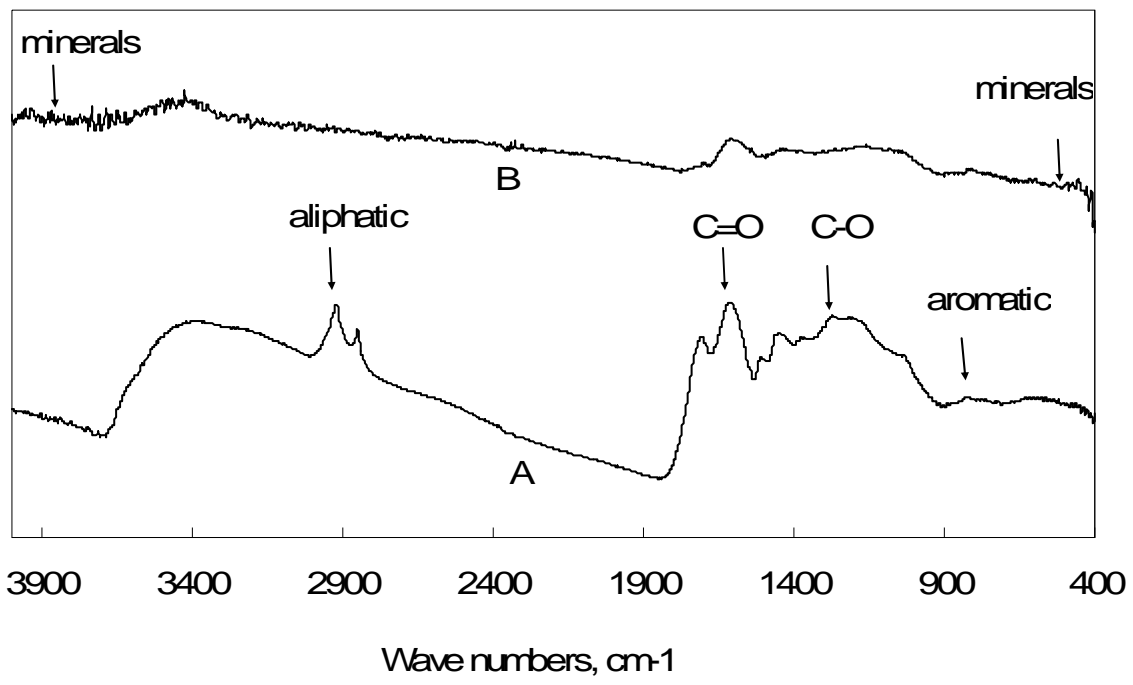

Figure 4.5. FTIR spectra of L1 (A) and its char produced at $823 \mathrm{~K}(\mathrm{~B})$ 


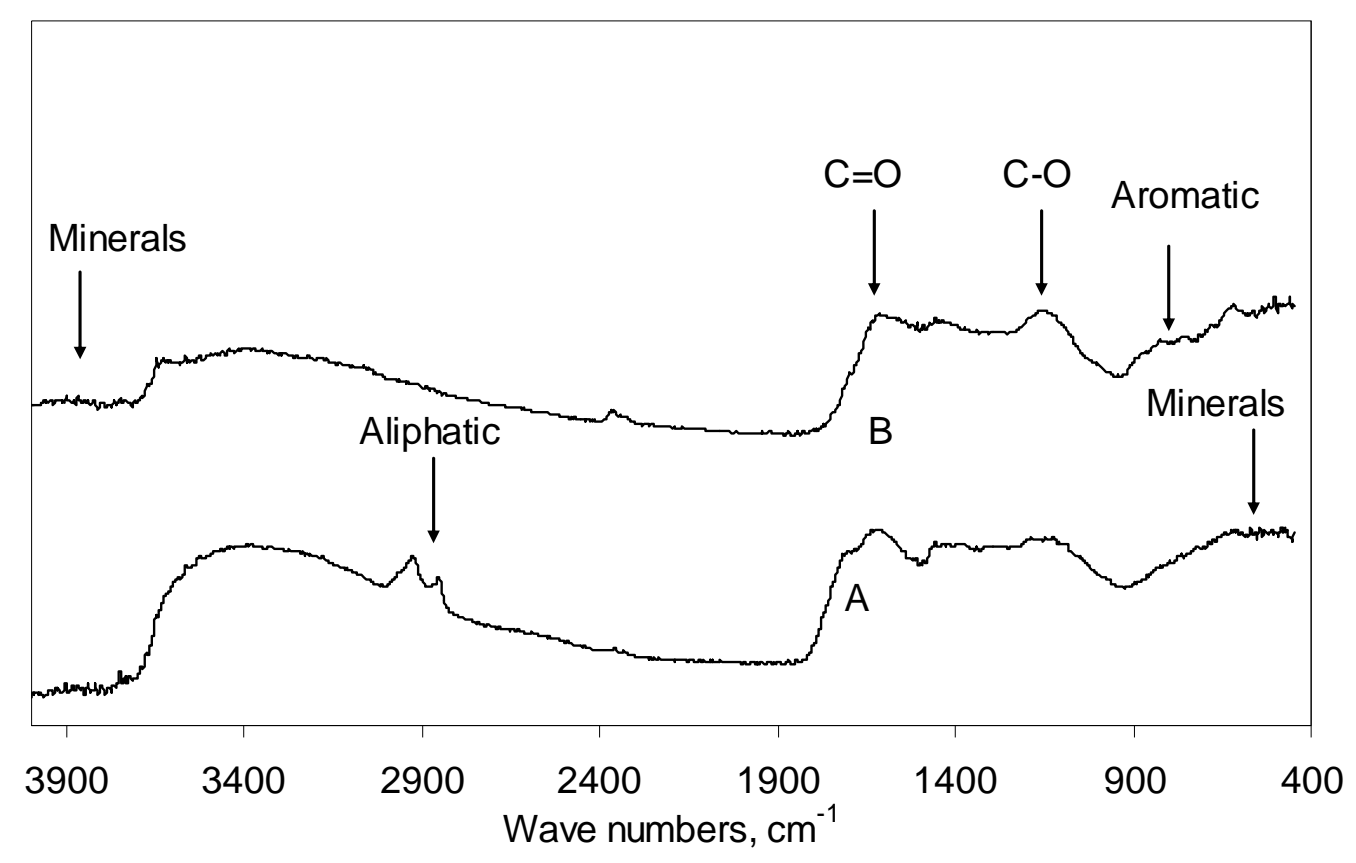

Figure 4.6. FTIR spectra of L2 (A) and its char produced at $823 \mathrm{~K}(\mathrm{~B})$

Figure 4.5 and 4.6 also show the FTIR spectra of L1 and L2 chars produced at $823 \mathrm{~K}$. The intensity of the characteristic bands of C-H and C-O decreases according to the loss of hydrogen and oxygen during devolatilisation [75, 87]. The broad $-\mathrm{OH}$ bands at around $3400 \mathrm{~cm}^{-1}$ is still observed in the chars, because some water still remain in the sample. The distinct peaks of $\mathrm{CH}$ stretching structure at about 2920 and $2850 \mathrm{~cm}^{-1}$ identified from raw L1 and L2 disappear from L1 and L2 chars. A peak at around 1600 $\mathrm{cm}^{-1}$ is present in L1 and L2 chars, the peak could be related to carbonyl groups as well as to the aromatic $\mathrm{C}=\mathrm{C}$ stretching vibration [18]. The band at around $1030 \mathrm{~cm}^{-1}$ which represent oxygenated functional groups due to stretching of $\mathrm{C}-\mathrm{O}$ from aliphatic ethers and alcohols show lower intensity at the chars. During pyrolysis, the oxygenated groups are released as $\mathrm{H}_{2} \mathrm{O}, \mathrm{CO}_{2}, \mathrm{CO}$, aldehydes, alcohols and acids [18]. The intensity of bands at $600-900 \mathrm{~cm}^{-1}$ of the chars is higher than their raw samples; this means that chars contain more aromatic carbon. The bands at 400-600 and 3600-4000 which represent minerals are still observed from the chars. 
To support the FTIR results presented in the previous paragraphs, solid state ${ }^{13} \mathrm{C}$ nuclear magnetic resonance $\left({ }^{13} \mathrm{C} \mathrm{NMR}\right)$ were applied for some of the lignite samples. Nuclear Magnetic Resonance (NMR) spectroscopy is one of the analytical methods used to study the molecular structure of coal [19]. The investigation of the coal and coal products structure using NMR requires little sample preparation and is in a direct and non-destructive way[90, 91].

There are many NMR techniques to analyse the structure of coal and coal products. The first NMR used on coals was ${ }^{1} \mathrm{H}$ broad-band NMR [92]. The first solid-state ${ }^{13} \mathrm{C}$ NMR study on coal was published in 1976, it was the first time the structure of coal was determined by a direct method [92]. With the development of NMR spectrometers' technology, it is now possible to study other nuclei in coals and coals products, such as, ${ }^{23} \mathrm{Na},{ }^{27} \mathrm{Al},{ }^{29} \mathrm{Si},{ }^{33} \mathrm{~S}$, and ${ }^{119} \mathrm{Sb}[19,93]$. However, in solid-state NMR studies of coals, ${ }^{13} \mathrm{C}$ NMR is often chosen as it provides direct structural information and is experimentally easy to obtain a good signal to noise ratio and good resolution $[19,92$, 94].

The combination of solid-state ${ }^{13} \mathrm{C}$ NMR spectroscopy with the cross-polarisation (CP) technique with high-power proton decoupling and magic angle spinning (MAS) technique has become important to investigate the coal and related carbonaceous materials structures in which the aromatic and aliphatic carbon bands are well separated [94-96]. The CP technique aims to avoid long relaxation delays required for normal free induction decay methods, while the MAS method removes chemical-shift anisotropy and provides more than $100 \mathrm{ppm}$ broadening for aromatic peaks [94]. If the field strengths in MAS are low, there are no spinning sidebands observed. However, if the MAS frequency is lower than the highest chemical-shift anisotropy, the spinning sidebands arise. To suppress these spinning sidebands, many techniques have been used, 
Chapter 4

for example, total suppression of spinning sidebands (TOSS) and phase-altered spinning sidebands (PASS). Dipolar Dephasing (DPDP) technique is used to separate the tertiary and quaternary aromatic $\mathrm{C}$ peaks; and also to separate the mobile groups of aliphatic $\mathrm{C}$ peaks $\left(\mathrm{CH}_{3}\right.$ and alkyl $\left.\mathrm{CH}_{2}\right)$ from more rigid $\mathrm{CH}_{2}$ and $\mathrm{CH}$ groups. To avoid overlapping signal, a DPDP method which only provides two spectra has been developed. The first spectrum contains the ordinary spectrum with all signals, while the second spectrum contains only quaternary $\mathrm{C}$ atoms and $\mathrm{CH}_{3}$ groups signals [19, 94].

Solid-state ${ }^{13} \mathrm{C}$ CP-MAS NMR and solid state ${ }^{13} \mathrm{C}$ TOSS NMR spectra of L2 are shown in Figure 4.7 and 4.8. The spectra are quite similar to typical of low rank coal spectra [97]. The ${ }^{13} \mathrm{C}$ CP-MAS NMR and ${ }^{13} \mathrm{C}$ TOSS NMR spectra fall into two well-separated groups, the aliphatic $\mathrm{C}$ group ( 0-60 ppm) and aromatic $\mathrm{C}$ region $(60-250 \mathrm{ppm})$ [92, 98]. ${ }^{13} \mathrm{C}$ TOSS NMR was used to correct spinning side bands. The aliphatic $\mathrm{C}$ regions after having suppressed of spinning sidebands (TOSS) show alkyl ( $33 \mathrm{ppm}$ ) and methyl ( 14 ppm) aliphatic groups. While in the aromatic $\mathrm{C}$ group, there are organic methyl (60 ppm), Aromatic-C-H groups ( 129 ppm) and quaternary carbons (147 ppm). The abundance of oxygenated structures which are observed from FTIR spectra, are also detected in the raw lignite sample from NMR analysis. The oxygenated structures identified are Aromatic-C-O- (156 ppm) and Aromatic-C(=O)-O- (179 ppm). Other spectra of raw lignites are presented in appendix, since the NMR spectra are quite similar. 


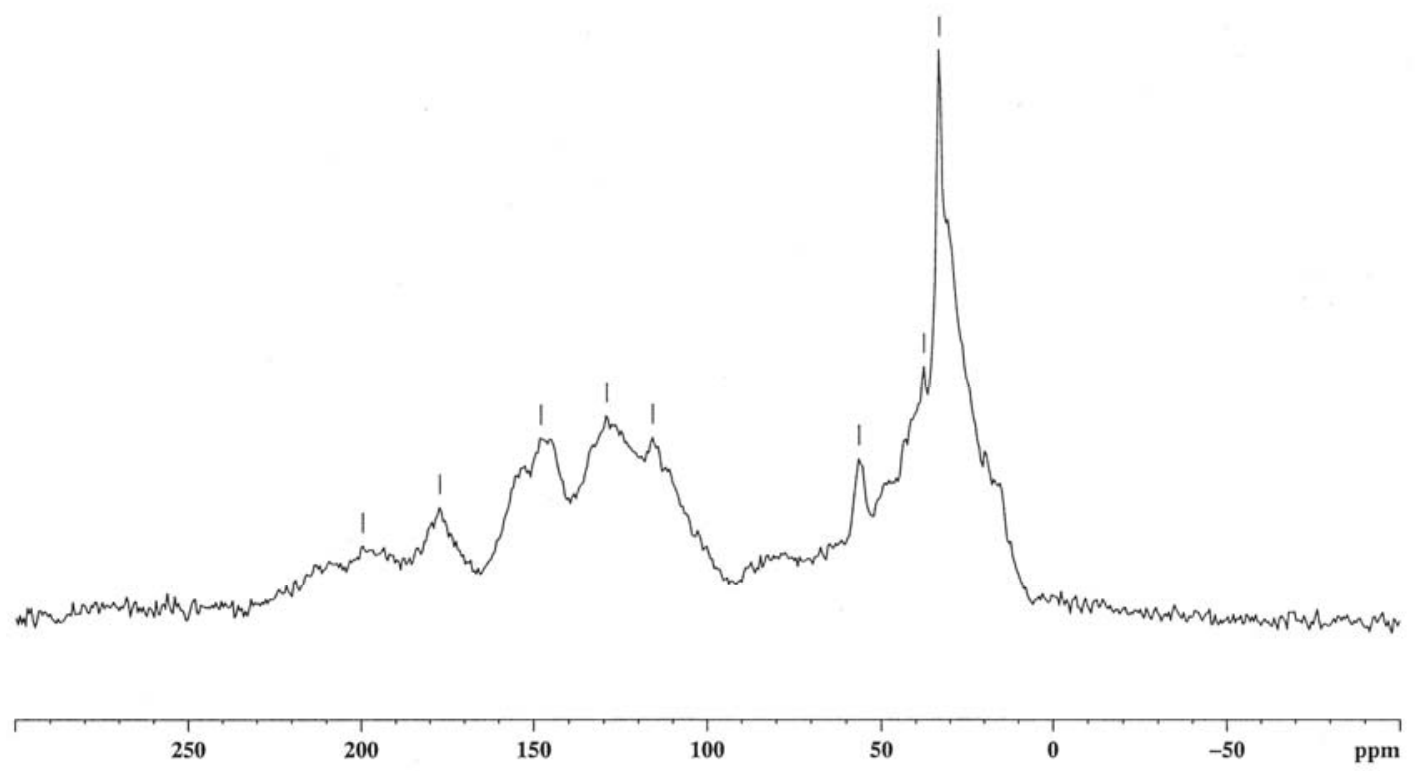

Figure 4.7 Solid-state ${ }^{13} \mathrm{C}$ CP-MAS of L1

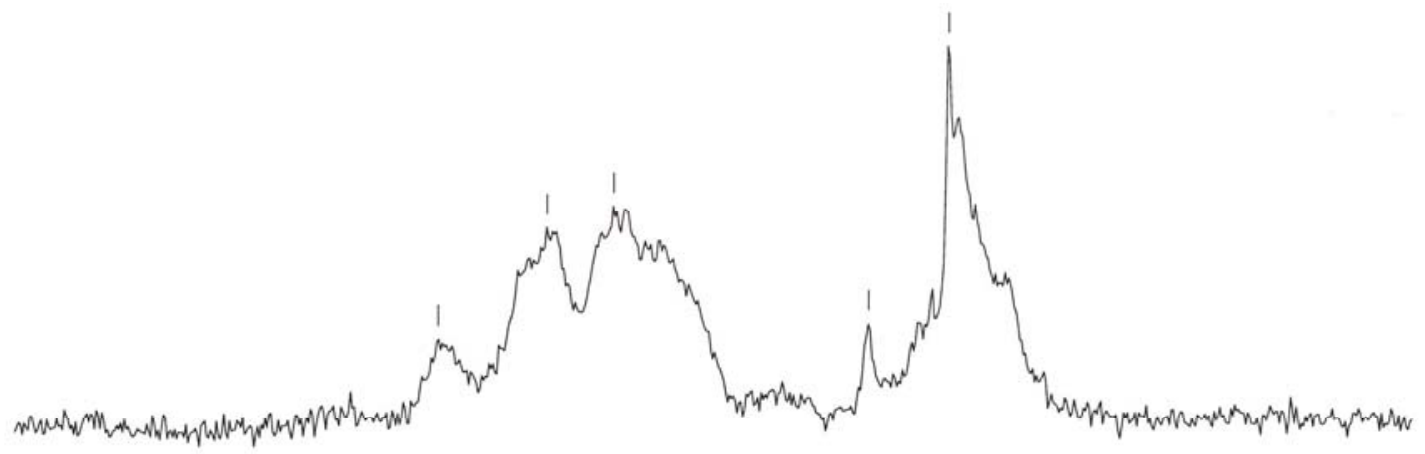

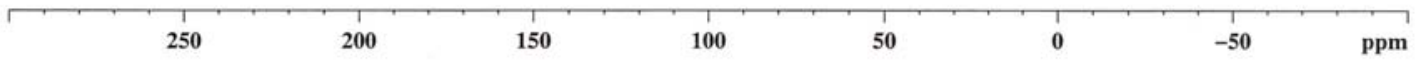

Figure 4.8 Solid-state ${ }^{13} \mathrm{C}$ TOSS spectra of L1

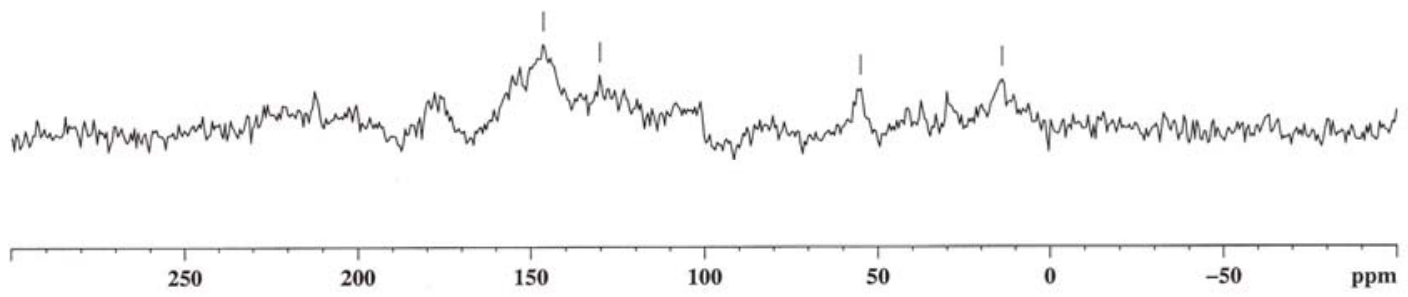

Figure 4.9 Solid-state ${ }^{13} \mathrm{C}$ DPDP spectra of L1 
To confirm the presence of aliphatic and aromatic groups solid state ${ }^{13} \mathrm{C}$ DPDP was applied to L1, the spectra is presented in Figure 4.9. Solid state ${ }^{13} \mathrm{C}$ DPDP NMR provides spectra which contain only methyl group and quaternary aromatic carbon group. From Figure 4.9 it can be seen the aliphatic and aromatic structures identified from L1; the aliphatic methyl group is centred at $14 \mathrm{ppm}$ and the aromatic methyl group is centred at $55 \mathrm{ppm}$, while quaternary carbon (Aromatic-C) is centred at 130, and $146 \mathrm{ppm}$.

Solid-state ${ }^{13} \mathrm{C}$ CP-MAS NMR and solid state ${ }^{13} \mathrm{C}$ TOSS NMR spectra of L2 were also applied for L1 char produced at $823 \mathrm{~K}$. Compare to its raw sample, the amount of aliphatic carbon strongly decreases for the char sample as shown in Figure 4.10. and 4.11. The loss of aliphatic carbon in the char samples is also confirmed by the FTIR results as already discussed on previous pages and shown in Figure 4.5 and 4.6; in those figures it is shown that the aliphatic stretching region is not present in the lignite chars. From Figure 4.10 and 4.11 it can be seen that the char is dominated by the aromatic carbon groups with the Aromatic-C-H groups (129 ppm) becoming more dominant. For the char, DPDP method was not used, because from Figure 4.10 and 4.11 the methyl groups are not too obvious detected. The NMR spectra of other lignite chars are shown in Appendix B. 


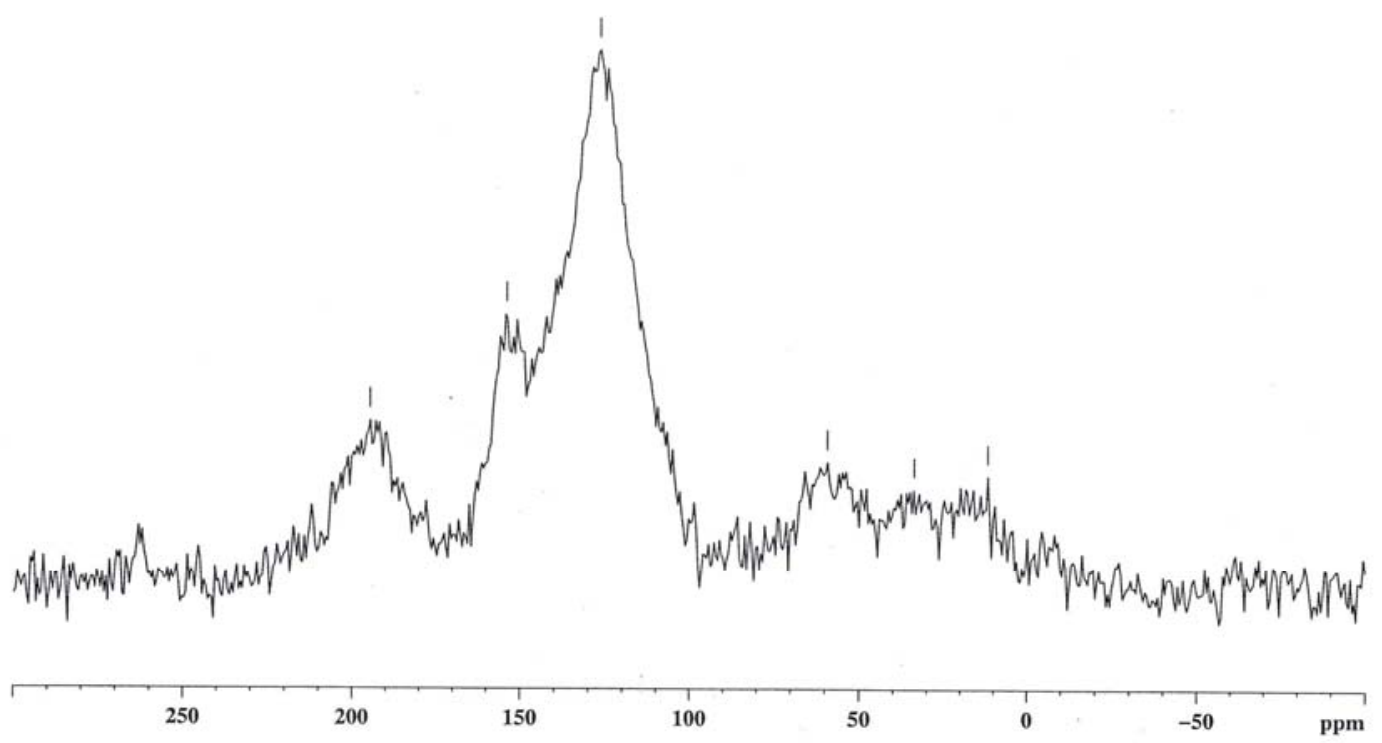

Figure 4.10 Solid-state ${ }^{13} \mathrm{C}$ CP-MAS spectra of Western Australian lignite char

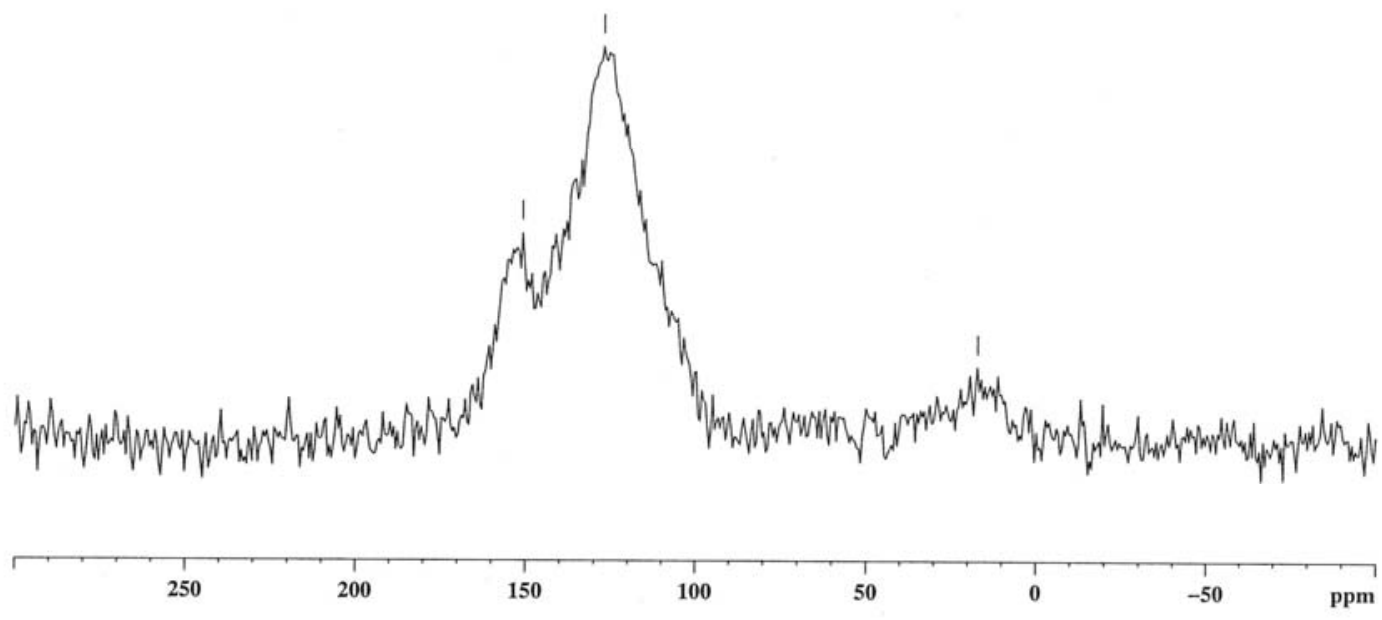

Figure 4.11 Solid-state ${ }^{13} \mathrm{C}$ TOSS spectra of Western Australian lignite char

The aromaticity, the ratio of the number of aromatic carbons to the total carbons (fa), is one of the key parameters to describe the coal structure [91]. The aromaticity of the lignite obtained from the ${ }^{13} \mathrm{C}$ CPMAS NMR spectra has been calculated by defining the aliphatic carbons $\left(\mathrm{C}_{\mathrm{al}}\right)$ as those with chemical shifts between 0 and $60 \mathrm{ppm}$ and the 
aromatic carbons $\left(\mathrm{C}_{\mathrm{ar}}\right)$ as those with chemical shift $>60 \mathrm{ppm}$. The aromaticity (fa) can be calculated as [19]:

$$
\begin{gathered}
f a=\frac{C_{a r}}{C_{\text {tot }}} \\
\mathrm{C}_{\text {tot }}=\mathrm{C}_{\mathrm{al}}+\mathrm{C}_{\mathrm{ar}}
\end{gathered}
$$

where $\mathrm{C}_{\mathrm{al}}$ is the sum of aliphatic carbon, $\mathrm{C}_{\mathrm{ar}}$ is the sum of aromatic carbon, $\mathrm{C}_{\text {tot }}$ is total carbon and fa is aromaticity.

Table 4.12 Aromaticity value of the lignites

\begin{tabular}{l|l}
\hline Samples & $\begin{array}{l}\text { Aromaticity } \\
(\mathrm{fa})\end{array}$ \\
\hline L1 & 0.62 \\
L2 & 0.49 \\
L3 & 0.49 \\
L5 & 0.48 \\
L7 & 0.52 \\
L10 & 0.62 \\
L11 & 0.47 \\
\hline
\end{tabular}

The results of fa for raw samples are presented in Table 4.12. Miknis et al [91] reported that carbon aromaticity obtained using CP/MAS NMR for lignite ranges from 0.6-0.7. Most of the samples have lower fa compared to the reported data, fa of the raw lignites ranges from $0.47-0.62$, thus the aliphatic groups are the dominant spectra of the raw lignites. However, it should be noted that fa values from NMR spectra is semi quantitative data [94]. 


\subsection{Petrographic analysis}

To study organic materials or commonly termed as macerals in lignite samples, petrographic analysis was carried out using a polished block of lignite as described at chapter 3 section 3.2.7.

Table 4.13. Maceral composition of the raw lignite

\begin{tabular}{lcccc}
\hline Samples & Rr[\%] & Min. & Max & Stdv. \\
\hline L1 & 0.21 & 0.17 & 0.27 & 0.03 \\
L2 & 0.22 & 0.16 & 0.26 & 0.03 \\
L3 & 0.19 & 0.17 & 0.22 & 0.02 \\
L4 & 0.2 & 0.15 & 0.28 & 0.03 \\
L5 & 0.25 & 0.2 & 0.3 & 0.04 \\
L6 & 0.19 & 0.14 & 0.21 & 0.02 \\
L7 & 0.25 & 0.21 & 0.25 & 0.04 \\
L8 & 0.27 & 0.23 & 0.3 & 0.02 \\
L9 & 0.25 & 0.21 & 0.31 & 0.03 \\
L10 & 0.18 & 0.16 & 0.19 & 0.02 \\
L11 & 0.25 & 0.2 & 0.35 & 0.04 \\
\hline
\end{tabular}

The petrographic composition of the raw lignites is summarised in Table 4.13. It can be seen that the raw lignites have random reflectance $(\mathrm{Rr})$ values ranging from $0.19 \% \mathrm{Rr}$ to $0.27 \% \mathrm{Rr}$. This reflectance range is similar to what Kortenski and Sotirov [99] reported for their lignites studied and according to ASTM D2798 lignites have reflectance values of less than $0.6 \%$ Rr. Since the random refelectance of lignites is usually less than $0.5 \%$ vitrinite does not occur, instead there is huminite maceral groups [23]. Huminite is the precursor of vitrinite [24]. Figure 4.12 shows the photomicrographs of the raw L2. It was found that all the lignites consist mainly of huminite (predominantly attrinite), it accounts more than $90 \%$ by volume, small amounts of inertinite (mainly funginite) about $1-2 \%$ by volume, and small amounts of semifusinite and liptinites (mainly resinite). 


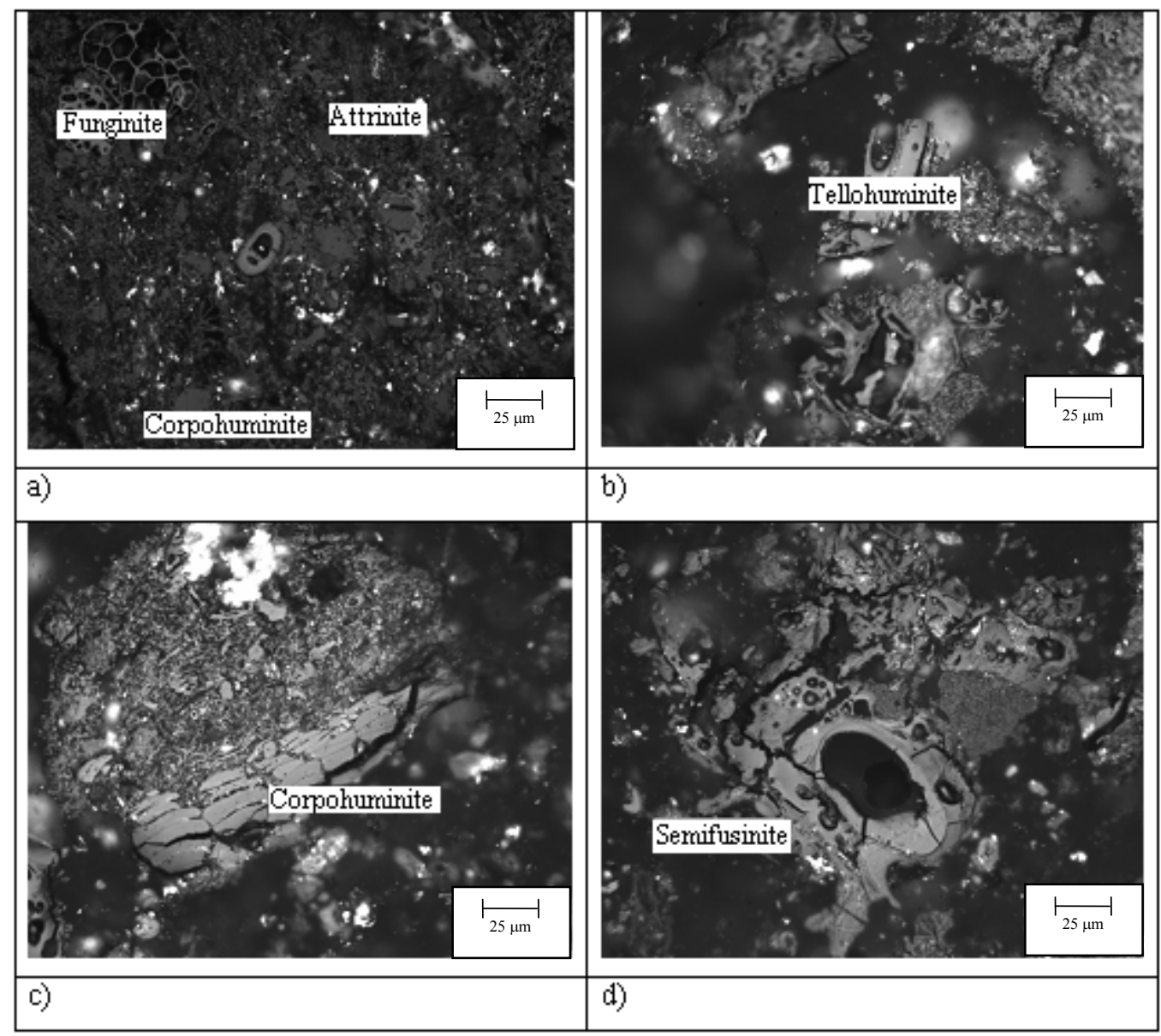

Figure 4.12 (a-d) Photomicrographs of raw L2

Table 4.14. Maceral composition of the lignite char

\begin{tabular}{lcccc}
\hline Samples & Rr[[\%] & Min. & Max & Stdv. \\
\hline Char L1 & 1.22 & 1.06 & 1.46 & 0.13 \\
Char L2 & 1.33 & 1.02 & 1.59 & 0.14 \\
Char L3 & 1.21 & 1.05 & 1.45 & 0.09 \\
Char L4 & 1.29 & 0.99 & 1.49 & 0.13 \\
Char L5 & 1.44 & 1.12 & 1.83 & 0.21 \\
Char L6 & 1.38 & 1.05 & 1.77 & 0.12 \\
Char L7 & 1.48 & 1.18 & 1.77 & 0.14 \\
Char L8 & 1.44 & 1.14 & 1.71 & 0.15 \\
Char L9 & 1.4 & 1.05 & 1.81 & 0.19 \\
Char L10 & 1.16 & 1.05 & 1.73 & 0.16 \\
Char L11 & 1.26 & 1.13 & 1.57 & 0.11 \\
\hline
\end{tabular}

Petrographic analysis was also conducted for lignite chars produced at $823 \mathrm{~K}$. The random reflectance values of chars is presented at Table 4.14. It can be seen that the charred samples have a much higher reflectance which is as expected since the heat 
treatment affects the maturation of the sample. The $\mathrm{Rr}$ of the chars ranges from $1.21 \% \mathrm{Rr}-1.48 \mathrm{Rr} \%$. Figure 4.13 shows the photomicrograph of char L2. It can be seen that inertinites remained unaltered in the thermal process while the liptinites evaporated.

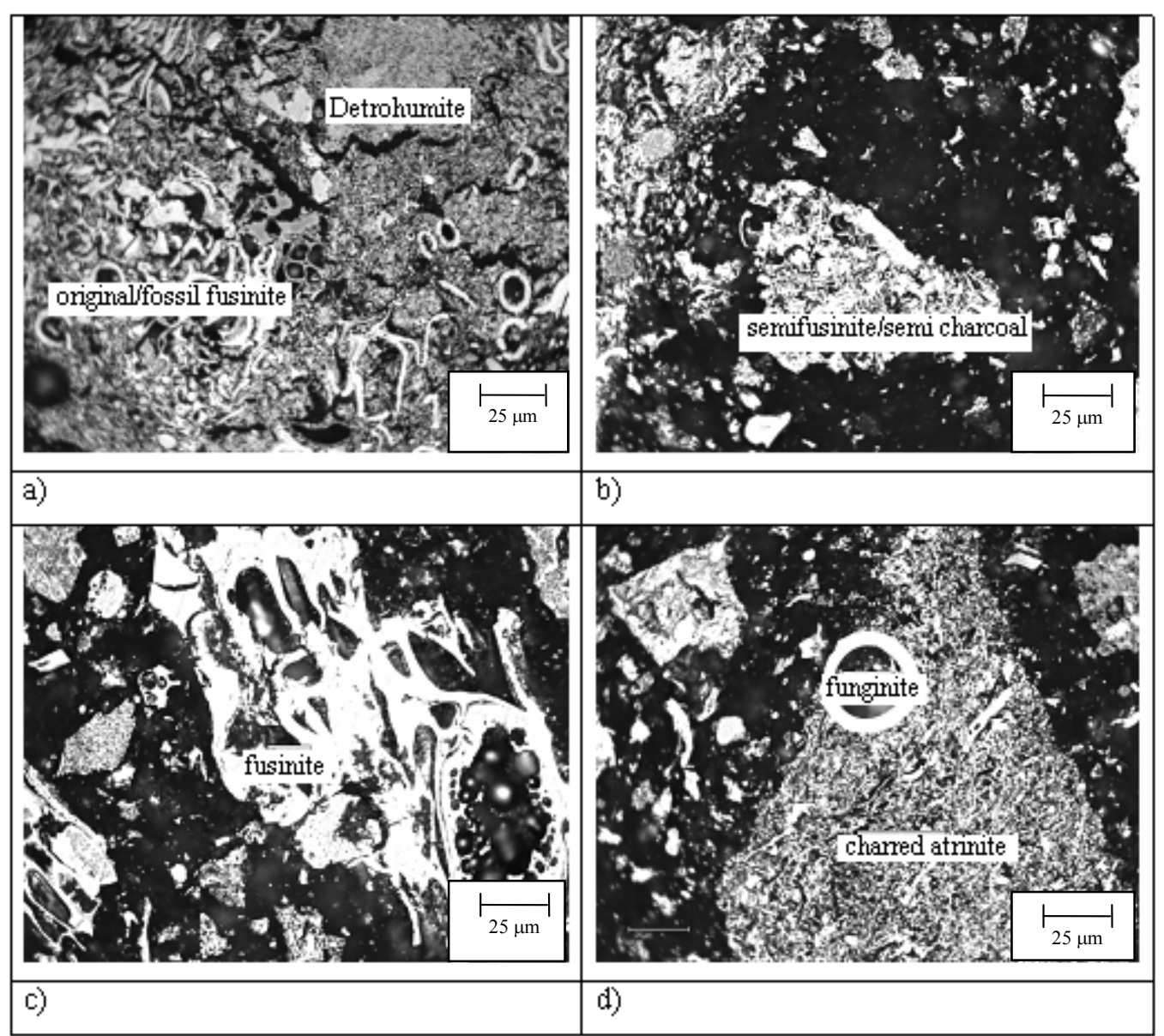

Figure 4.13 (a-d) Photomicrographs of L2 char

\subsection{Summary}

A combination of techniques has been used to characterise Australian lignites. The raw lignites are categorised as of low quality since they have very high moisture contents, ranging from 53.6-64.9\%, very high ash contents, ranging from 20.7-32.7\% (except for L1 which only contains $3.1 \%$ ash), low fixed carbon, ranging from $24.6-44.3 \%$, thus they have low calorific values, ranging from 17.8-26.6 MJ. $\mathrm{kg}^{-1}$. 
Chapter 4

The lignites contain a considerable amount of total sulphur, ranging from 1.37-4.62\%, except for L1 which only has $0.24 \%$ total sulphur. L1 is also pyrite-free lignite, it only contains $0.21 \%$ organic sulphur and $0.03 \%$ sulphate sulphur. L2 is the sample with the highest total sulphur, this lignite contain significant amount of pyritic, sulphate and organic sulphur. L3 and L5-L11 are the lignites with low sulphate, low pyritic sulphur and high organic sulphur, while L4 is the lignite which contains high sulphate and low pyritic sulphur and very low organic sulphur.

Mineralogy of lignites has been studied using XRD on LTA of raw lignites and XRD and XRF analysis of HTA of lignites samples. The results of the LTA analyses show that LTA is not suitable to study the mineralogy of lignite since the sulphate compounds identified are not the sulphates really present in the samples but seem artefact of the LTA process. XRD analysis shows that the HTA of the lignites are dominated by $\mathrm{NaCl}$, while XRF analysis shows that the high temperature ashes predominantly contain sodium followed by sulphur. Fe, $\mathrm{Si}, \mathrm{Al}$ and $\mathrm{Mg}$ are also present in significant amounts for some of the lignites.

Most of the sodium and chlorine present in the raw lignites are water soluble sodium and chorine, thus in the form of $\mathrm{NaCl}$. From the IC analysis it is obtained that the raw lignites contain extremely high $\mathrm{NaCl}$, ranging from6.61-19.39\%, except for $\mathrm{L} 1$ which only contains $0.13 \% \mathrm{NaCl}$.

FTIR and solid state ${ }^{13} \mathrm{C}$ NMR spectroscopy studies show that aliphatic carbon presents significantly in the samples. It is confirmed with fa obtained for the raw samples, ranging from 0.47-0.62. The oxygenated functional structures are also identified significantly in the raw samples; it shows with the intense peak between $1000-1800 \mathrm{~cm}^{-}$ ${ }^{1}$ of FTIR spectra and Aromatic-C-O- (156 ppm) and Aromatic-C(=O)-O- (179 ppm) of 
Chapter 4

the NMR spectra. Both FTIR and NMR spectra show that the chars have more dominant aromatic carbon than the raw lignites.

Petrographic analysis shows that the raw lignites are dominated by huminite, the precursor of vitrinite. The $\mathrm{Rr}$ values of the raw lignites range between $0.19 \% \mathrm{Rr}$ to $0.27 \% \mathrm{Rr}$. The heat treatment affects the maturation of the sample, it is confirmed with the $\mathrm{Rr}$ of the chars ranges from $1.21 \% \mathrm{Rr}-1.48 \mathrm{Rr} \%$.

From the characterisation of the lignites, the samples needed for the study of sulphur transformation during lignite pyrolysis can be chosen. To study the effect of inherent mineral and inorganic matter, both raw and acid washed samples have been used during lignite pyrolysis. AW L1, the pyrite and mineral free lignite, was chosen as the sample employed for pyrite, sulphate and organic sulphur added lignite. While L3, the lignite with high organic sulphur and high mineral and inorganic matter content, is chosen as the lignite to be added with inorganic matter to study the effect of inherent and added inorganic matter on the sulphur transformation during lignite pyrolysis. Since the lignites contain high oxygenated structures, it is worthwhile to study the effect of high oxygenated structures on the sulphur transformation during lignite pyrolysis.

To study the pyrite transformation, L1, AW L1, L2, AW L2 and mineral pyrite blended with AW L1 in various concentrations have been used, and the pyrite transformation is discussed in detail in chapter 5.

Sulphate transformation has been studied by adding $\mathrm{CaSO}_{4}$ and $\mathrm{FeSO}_{4}$ to $\mathrm{AW} \mathrm{L1}$. Lignite with high sulphate content, L4, was also used in the experiment. The sulphate transformation during lignite pyrolysis will be discussed in detail in Chapter 6 . 
Chapter 4

Organic sulphurs have been added to AW L1, the behaviour of organic sulphur in the samples along with lignite samples which contain high organic sulphur have been studied. The details are described in Chapter 7.

To study the effect of inherent and added inorganic matter on the sulphur transformation during lignite pyrolysis, sodium and kaolinite have been added to AW L3, the details are explained in Chapter 8. 


\section{CHAPTER 5}

\section{Pyrite Transformation during Lignite Pyrolysis}

\subsection{Introduction}

In freshly mined coals, most of the sulphur is in the form of pyrite $\left(\mathrm{FeS}_{2}\right)$ [4]. The presence of pyrite affects coal utilisation processes due to sulphur dioxide $\left(\mathrm{SO}_{2}\right)$ emission in combustion processes or hydrogen sulphide $\left(\mathrm{H}_{2} \mathrm{~S}\right)$ and COS formation in gasification processes, requiring additional emission control measures or clean-up [100]. On the other hand, pyrite is also known to catalyse coal reactions, including oxidation, gasification and liquefaction processes [4, 101-103]. However, it is not clear as yet how pyrite exerts its catalytic effect.

Pyrolysis is one of the first steps in many thermo-chemical utilisation processes of coal. During pyrolysis, pyrite in coal can be transformed into ferrous sulphide at temperatures above $633 \mathrm{~K}$ and very active sulphur. This active sulphur can react with hydrogen in the coal to form hydrogen sulphide or react with active sites of organic matrix on char surfaces to form new organic sulphur species $[7,8]$.

This chapter presents and discuses the transformation of pyrite and its interactions with various organic and inorganic species during pyrolysis of Australian lignites. It has been discussed in Chapter 4 that some of the lignites contain significant but varying amounts of pyrite, a major factor impeding its utilisation.

In this work, the thermal behaviour of a pyrite mineral, two lignite samples, and lignite blended with various amounts of the pyrite were studied using a TGA. Thermogravimetric analysis (TGA) was used to investigate the thermal behaviour of 
Chapter 5

pyrite reduction and to obtain the kinetics of the lignite pyrolysis [58, 104-107]. The volatile products during pyrolysis of the lignite were analysed using a TGA coupled to a mass spectrometer (MS). Pyrolysis experiments were also conducted in a fixed bed reactor so that the sulphur transformation, especially pyrite transformation, could be studied using Fourier transfom infrared (FTIR), Scanning electron microscope equipped with an energy-dispersive X-ray spectroscopy (SEM-EDS) and X-ray diffraction (XRD) analyses on the resultant chars.

\subsection{Results and Discussion}

\subsubsection{Thermal behaviour of pyrite in TGA Pyrolysis}

The mass loss profile during the pyrolysis of the pyrite mineral in nitrogen is shown in Figure 5.1, where the S/Fe ratio calculated based on the mass loss profile is also plotted. There are broadly four stages in the pyrolysis of a pure pyrite. Stage 1 occurs below ca. $720 \mathrm{~K}$ during which only a very small mass loss is observable, due to probably only the absorbed gases being released since the $\mathrm{S} / \mathrm{Fe}$ ratio is still very close to 2 . Stage 2 occurs between ca. 720 and ca. $845 \mathrm{~K}$ during which the rate of mass loss is clearly increased, indicating that the pyrite is being decomposed. At this stage the minimum $\mathrm{S} / \mathrm{Fe}$ ratio achieved for pyrite decomposition at $10 \mathrm{~K} \cdot \mathrm{min}^{-1}$ heating rate is 1.82 and at $50 \mathrm{~K} \cdot \mathrm{min}^{-1}$ heating rate is 1.87 . Stage 3 occurs between ca. 845 and $940 \mathrm{~K}$ during which the sample experiences very rapid mass loss. In Stage 3 it can be observed from Figure 5.1 that it has $1.82 \leq \mathrm{S} / \mathrm{Fe} \leq 1.11$ for $10 \mathrm{~K} \cdot \mathrm{min}^{-1}$ heating rate and $1.87 \leq \mathrm{S} / \mathrm{Fe} \leq 1.13$ for $50 \mathrm{~K} \cdot \mathrm{min}^{-1}$ heating rate, respectively. Finally, in Stage 4 at temperature above $940 \mathrm{~K}$, the mass loss rate is dramatically slowed. In this stage, the $\mathrm{S} / \mathrm{Fe}$ ratio for the heating rate of $10 \mathrm{~K} \cdot \mathrm{min}^{-1}$ is between 1.11 to 0.98 and, for the heating rate of $50 \mathrm{~K} \cdot \mathrm{min}^{-1}$, between 1.13 to 1.01 . 
The variation in the $\mathrm{S} / \mathrm{Fe}$ ratio was small between two heating rates. The fact that it did vary suggests that the pyrite pyrolysis is a kinetic controlled process.

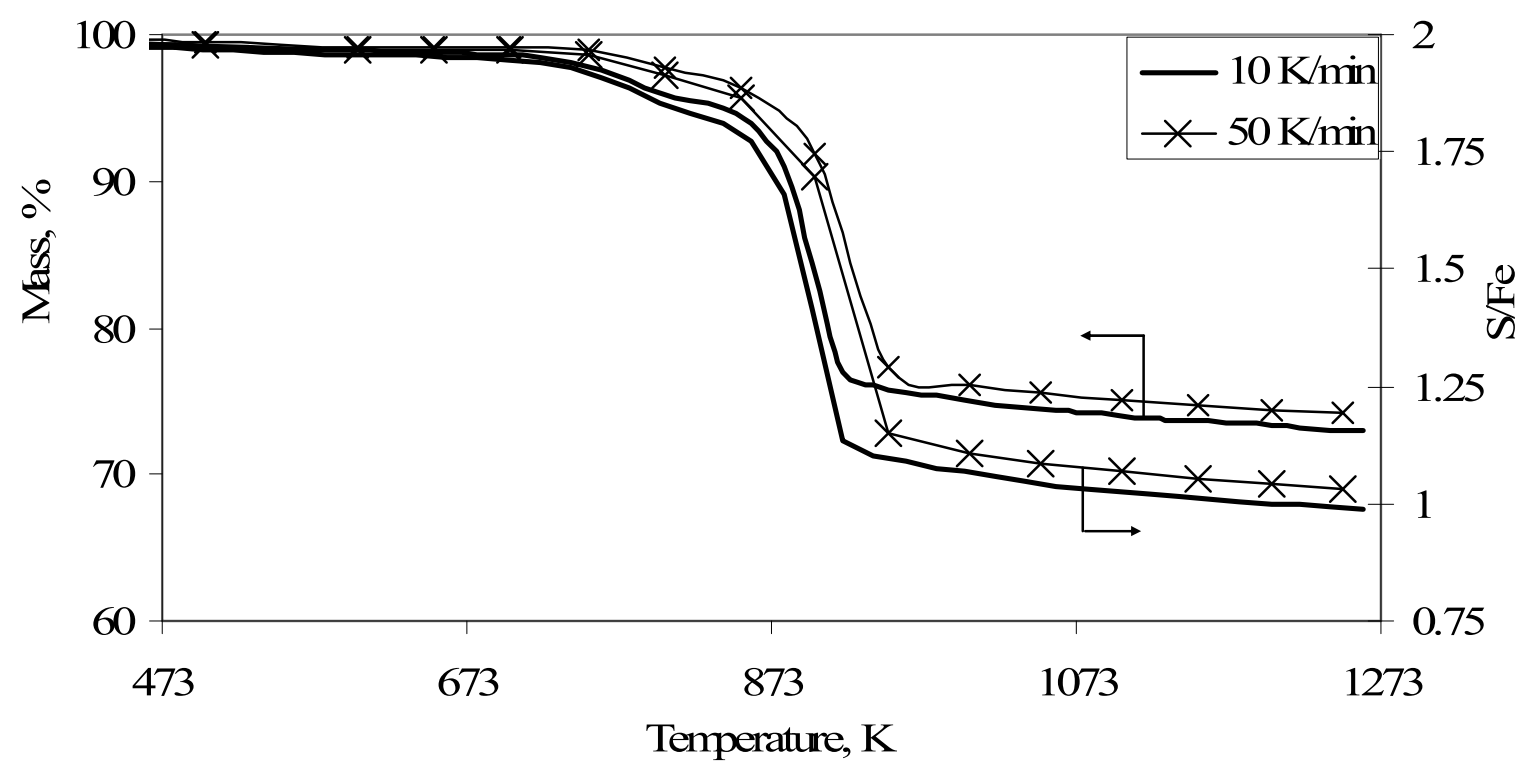

Figure 5.1.TGA mass loss and Fe/S ratio profiles of pyrite decomposition

In an inert atmosphere pyrite decomposes via a multi-step sequential process [51], i.e.

$$
\mathrm{FeS}_{2} \rightarrow \mathrm{FeS}_{\mathrm{x}} \rightarrow \mathrm{FeS} \rightarrow \mathrm{Fe}
$$

Complete decomposition of pyrite to troilite (FeS) should incur $26.7 \%$ mass loss and $73.3 \%$ of the initial mass should be retained. From Figure 5.1, it can be seen that $\mathrm{FeS}_{2}$ ( $\mathrm{S} / \mathrm{Fe}$ ratio 2) is completely decomposed to form $\mathrm{FeS}$ when $\mathrm{Fe} / \mathrm{S}$ ratio is 1.0 at ca 1200 $\mathrm{K}$ when the TGA was run at $10 \mathrm{~K} \cdot \mathrm{min}^{-1}$ heating rate. At $50 \mathrm{~K} \cdot \mathrm{min}^{-1}$, however, the pyrite does not achieve complete decomposition even at $1250 \mathrm{~K}$, indicating that the pyrolysis of pyrite is a kinetically controlled process. Before complete decomposition, the iron sulphide is present in a mixed form of pyrrothite $\left(\mathrm{FeS}_{\mathrm{x}}\right.$ with $\left.1<\mathrm{x}<2\right)$. These observations are supported by data from the SEM-EDS analysis. The atomic ratio of $\mathrm{S} / \mathrm{Fe}$ of the raw pyrite detected by SEM-EDS is exactly 2, the atomic ratio of $\mathrm{S} / \mathrm{Fe}$ of the decomposed samples collected between $773-1173 \mathrm{~K}$ are $1<\mathrm{S} / \mathrm{Fe}<2.0$, while the $\mathrm{S} / \mathrm{Fe}$ ratios of the 
two decomposed samples recovered from the TGA following the pyrolysis experiments to $1273 \mathrm{~K}$ give different $\mathrm{S} / \mathrm{Fe}$ ratios; the sample run at $50 \mathrm{~K} \cdot \mathrm{min}^{-1}$ has $\mathrm{S} / \mathrm{Fe} \sim 1.0$, while the sample run at $10 \mathrm{~K} \cdot \mathrm{min}^{-1}$ has $\mathrm{S} / \mathrm{Fe}<1.0$.

A close inspection of Figure 5.1 reveals that the mass loss continues above $1200 \mathrm{~K}$ in the $10 \mathrm{~K} \cdot \mathrm{min}^{-1}$ TGA experiment, suggesting that some troilite has been decomposed to elemental iron $(\mathrm{Fe})$. The $\mathrm{S} / \mathrm{Fe}$ ratio above $1200 \mathrm{~K}$ at a heating rate $10 \mathrm{~K} \cdot \mathrm{min}^{-1}$ is $<1.0$.

Gaseous sulphur is released during the thermal decomposition of pyrite. The sulphur gas is usually in the form of a mixture of allotropic species, $S_{n}$, with $n$ varying from 1 to 8 [51]. The fraction of each allotropic species in the mixture is determined by thermodynamic equilibrium. $\mathrm{S}_{2}$ is the dominant allotropic species for the decomposition of pyrite at temperatures less than $800 \mathrm{~K}$, while $\mathrm{S}_{3}, \mathrm{~S}_{5}$ and $\mathrm{S}_{6}$ in the allotropic mixture are only significant at higher temperatures. Considering that the sulphur gas is a mixture of allotropic species and the pyrite decomposition is a reversible process as the pyrite/pyrrothite can be synthesised from the sulphidation reaction between iron and sulphur gas [51], the overall reaction of pyrite decomposition (R.1) can be broken down as follows:

$$
\begin{aligned}
& \mathrm{FeS}_{2(\mathrm{~s})} \leftrightarrow \mathrm{FeS}_{\mathrm{x}(\mathrm{s})}+1 / \mathrm{n}(2-\mathrm{x}) \mathrm{S}_{\mathrm{n}(\mathrm{g})} \\
& \mathrm{FeS}_{\mathrm{x}(\mathrm{s})} \leftrightarrow \mathrm{FeS}_{(\mathrm{s})}+1 / \mathrm{n}(\mathrm{x}-1) \mathrm{S}_{\mathrm{n}(\mathrm{g})} \\
& \mathrm{FeS}_{(\mathrm{s})} \leftrightarrow \mathrm{Fe}_{(\mathrm{s})}+1 / \mathrm{n} \mathrm{S}_{\mathrm{n}(\mathrm{g})}
\end{aligned}
$$

\subsubsection{Thermal behaviour of lignite and lignite - pyrite blends}

Two lignite samples with different pyrite contents, namely L1 and L2 were used in the experimentation. As discussed in Chapter 4, L1 contains no pyrite, very low sulphate sulphur $(0.03 \%)$ and low organic sulphur $(0.21 \%)$, while L2 has significant amounts of 
Chapter 5

pyrite $(1.61 \%)$, sulphate sulphur $(1.27 \%)$ and organic sulphur $(1.74 \%)$. The acid washed samples were also studied; they were named AW L1 and AW L2, respectively. Table 5.1 shows total, organic and inorganic sulphur contents of the lignites and their demineralised samples. Acid washing was carried out to remove acid soluble inorganic presents in the lignite. From Table 5.1 it can be seen that the inorganic sulphur in L2 is $2.88 \%$, and in AW L2 is $1.66 \%$. Most of the sulphate in L2 is acid soluble which is washed away during the acid washing, thus the inorganic sulphur in AW L2 is dominated by pyritic sulphur.

Table 5.1 Total, organic and inorganic sulphur contents of the lignite samples

\begin{tabular}{|l|l|l|l|}
\hline Sample & Total S, \%db & Organic S, \% & Inorganic S, \%db \\
\hline L1 & 0.24 & 0.21 & 0.03 \\
\hline AW L1 & 0.21 & 0.21 & - \\
\hline L2 & 4.62 & 1.74 & 2.88 \\
\hline AW L2 & 3.4 & 1.74 & 1.66 \\
\hline
\end{tabular}

Lignite blended with various amount of pyrite minerals were also used. AW L1, a demineralised pyrite-free lignite sample, was chosen to be blended with three different concentrations of pyrite, they were 5P-95L1 (5\% pyrite $+95 \%$ AW L1), 10P-90L1 $(10 \%$ pyrite $+90 \%$ AW L1), and 50P-50L1 (50\% pyrite $+50 \%$ AW L1), respectively.

The mass loss profiles and differential thermogravimetry (DTG) thermograms during the pyrolysis of L1, AW L1, L2 and AW L2 at a heating rate of $50 \mathrm{~K} \cdot \mathrm{min}^{-1}$ from $373 \mathrm{~K}$ to $1073 \mathrm{~K}$ on dry basis are presented in Figure.5.2. From Figure 5.2 it can be seen that L2, the lignite with high ash content and its demineralised sample (AW L2) have quite different char yield on dry basis, whereas lignite with low ash content (L1) and its demineralised sample (AW L1) have rather similar char yield at the same pyrolysis temperature. Therefore, to study the effect of pyrolysis temperature on the char yield 
Chapter 5

without the interference of inorganic matter, the TGA mass loss profiles for pyrolysis of lignites are also presented on dry ash free (daf) basis and shown in Figure 5.3. It can be seen that the mass loss profile for L1 and L2 and their demineralised samples is quite similar. Therefore, the big difference of the remaining chars of L2 and AW L2 as shown in Figure 5.2 is because some of the mineral and inorganic is not volatilised during pyrolysis and the inorganic matter remains in the solid, affect the char yield on dry basis.

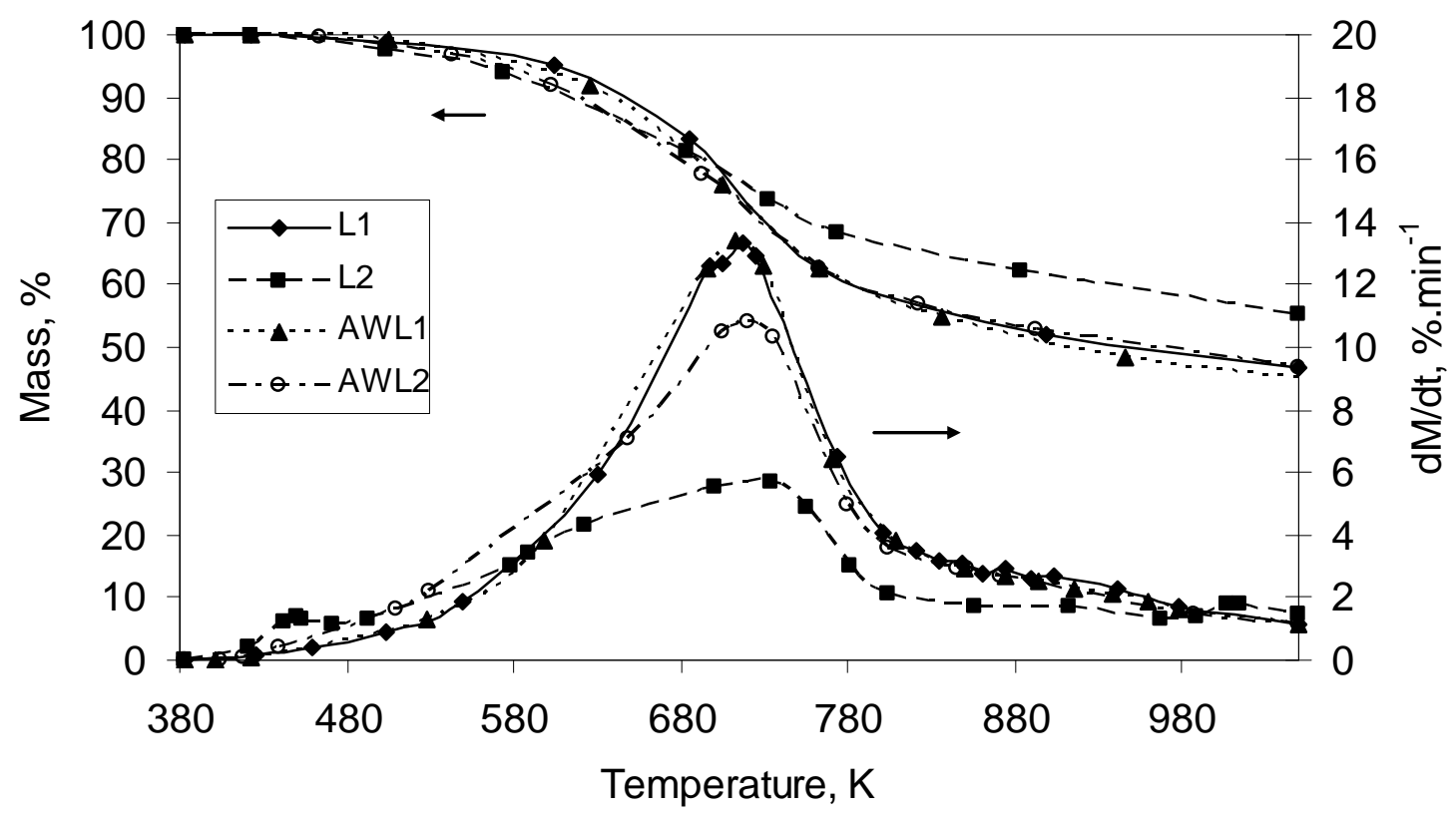

Figure 5.2.TGA mass loss profiles and DTG thermograms on dry basis of L1, AW L1, L2 and AW L2 during pyrolysis 


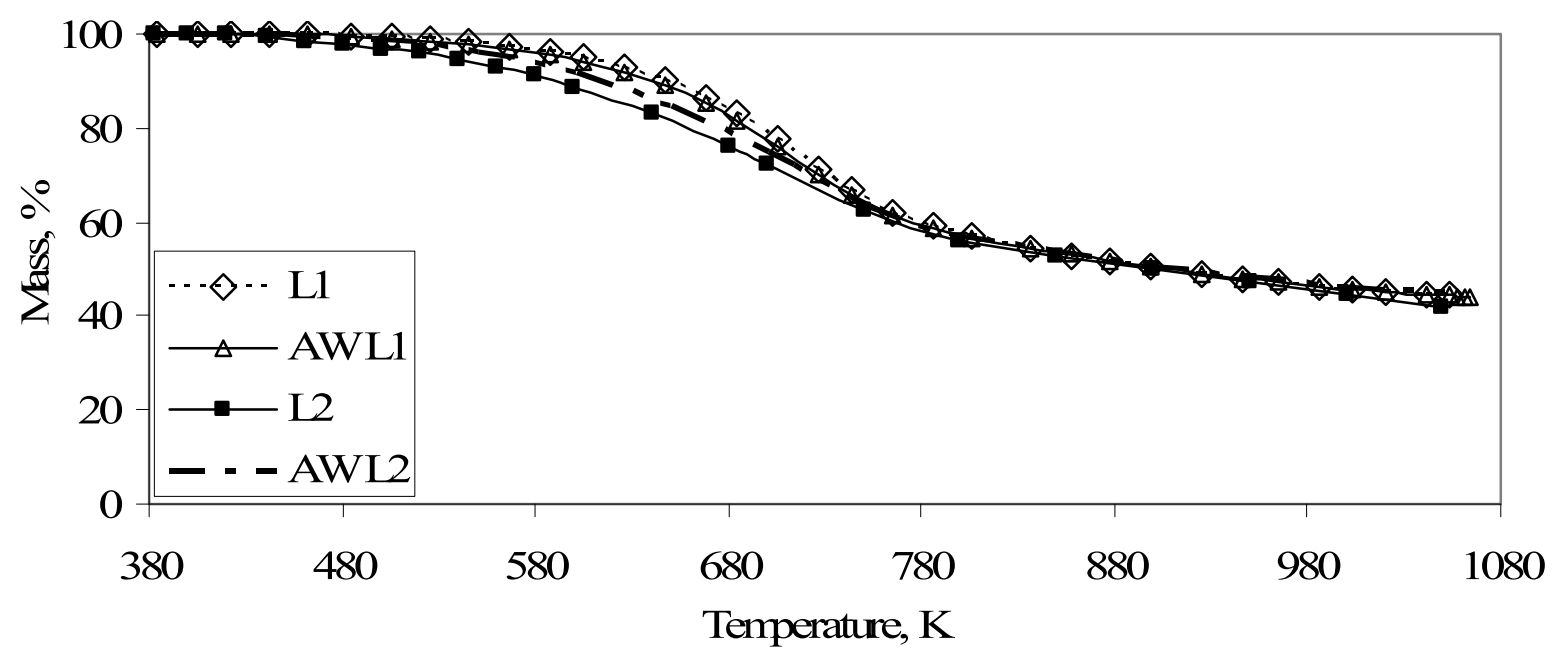

Fig 5.3. TGA mass loss profiles on dry ash free (daf) basis of L1, AW L1, L2 and AW L2 during pyrolysis

From Figure 5.3 it can be observed that there is a very little different of the char yield between L1 and AW L1. At the highest pyrolysis temperature (1073K), the char yield of L1 is $44.30 \%$ daf and AW L1 is $44.01 \%$ daf. For L2 and AW L2, the char yield difference on dry ash free basis is greater, at $1073 \mathrm{~K}$ the char yield of L2 is $41.80 \%$ daf and AW L2 is $44.86 \%$ daf. L1 is the pyrite-free lignite, while L2 contains a considerable amount of pyrite. The difference of the char yields from L2 and its demineralised sample could be related to the interaction between pyrite and organic matrix of the lignite, this leads to the retention of sulphur in solid phase.

From the samples presented in Figure 5.2, L2 has a secondary DTG peak, while other samples show only a single peak. L1 and AW L1 have very similar DTG peaks. L1, as mentioned previously, has very low minerals, and therefore after acid washing the acid washed sample (AW L1) has quite similar behaviour to its unwashed sample. However, L2, which has $27.1 \%(\mathrm{db})$ of the mineral matter, has a significantly lower height of the primary DTG peak compared to its acid washed sample (AW L2). From Figure 5.2 it can be seen that the lower the mineral matter in the sample, the higher the primary DTG 
Chapter 5

peak. AW L2 has only the single DTG peak, while L2 has both primary and secondary peaks, the secondary DTG peak of L2 could only be related to the decomposition of mineral matter present in L2, in this case, primarily the pyrite present in L2. To study the effect of pyrite during lignite pyrolysis, pure pyrite was added to AW L1, the pyritefree and almost mineral-free sample.

The mass loss profiles and differential thermogravimetry (DTG) thermograms during pyrolysis of AW L1 and lignite-pyrite blends in the TGA experiments at a heating rate of $50 \mathrm{~K} \cdot \mathrm{min}^{-1}$ from $373 \mathrm{~K}$ to $1073 \mathrm{~K}$ are presented and compared with that of pyrite in Figure 5.4. Significant mass losses occur for all samples in the temperature range between $573-923 \mathrm{~K}$, except for the pyrite sample which experienced a dramatic mass loss at higher temperatures between 845 and $940 \mathrm{~K}$.

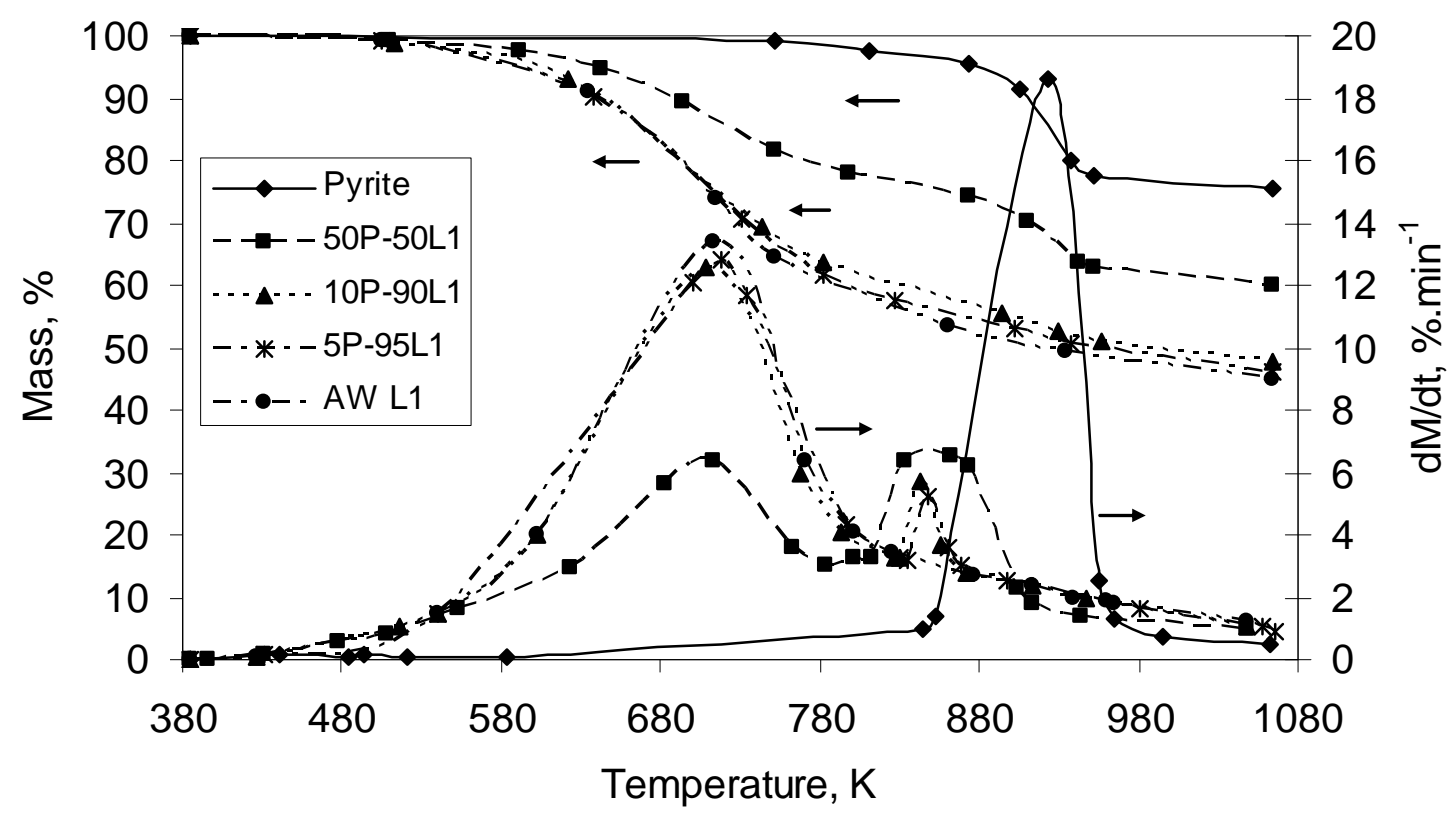

Figure 5.4.TGA mass loss profiles and DTG thermograms on dry basis during pyrolysis of pyrite, AW L1 and lignite-pyrite blends 


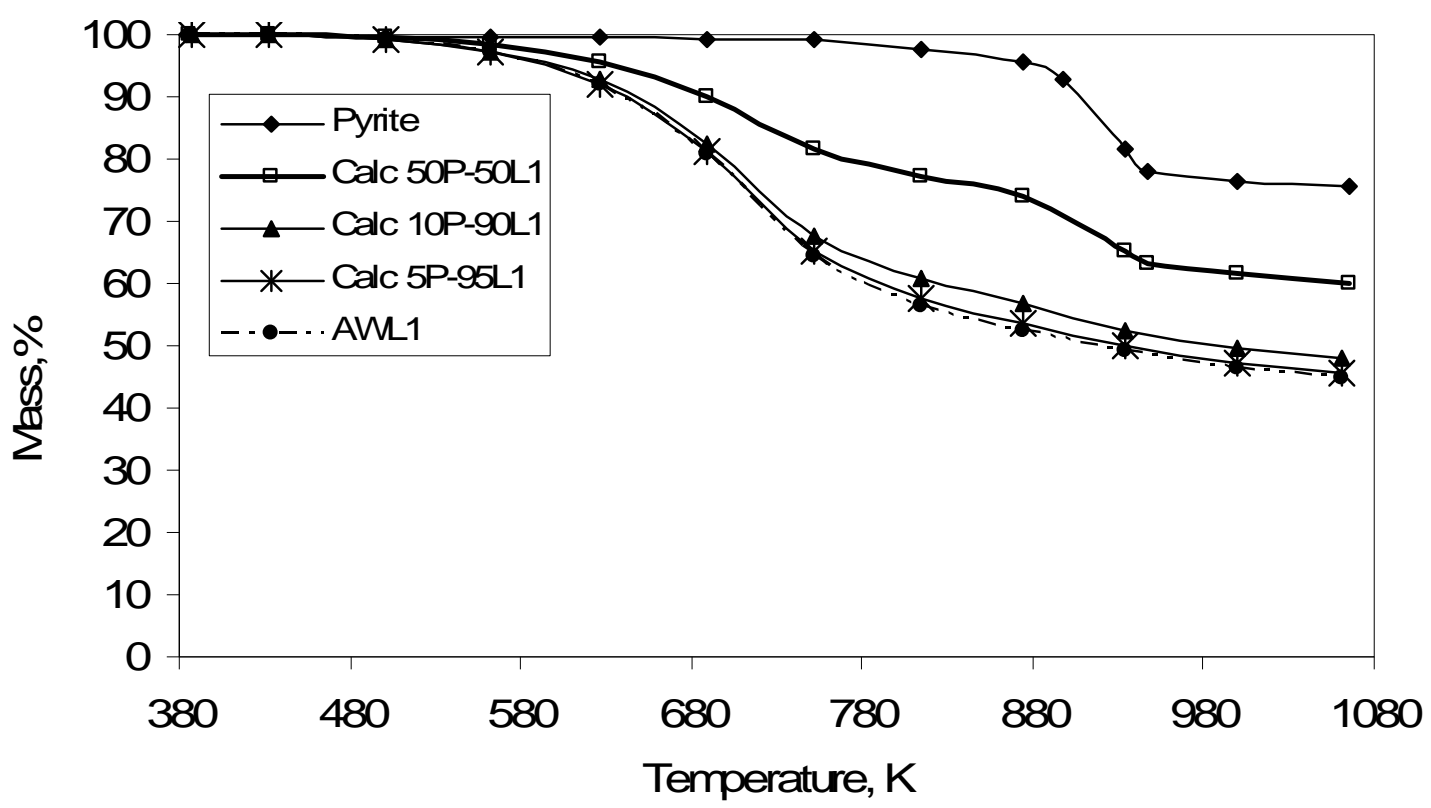

Figure 5.5. Calculated mass loss profiles (dry basis) during the pyrolysis of pyrite, AWL1 and their blends

To further study the effect of pyrite on the mass loss during pyrolysis of blended samples, the percentages of mass loss of the blended samples at the different pyrolysis temperatures were calculated based on the pyrolysis data of the pyrite mineral and AW L1 TGA. The results of the mass loss calculations for 5P-95L1, 10P-90L1 and 50P50L1 along with the TGA data of the pyrite and AW L1 are presented in Figure 5.5. It can be observed that the calculated data of blended samples behave very similar to the experimental data for the blended samples presented in Figure 5.4.

A further inspection of the TGA results presented in Figure 5.4 reveals that the blended samples also have two DTG peaks, a primary peak and a secondary peak, whereas the AW L1 and pyrite samples only have a single DTG peak, respectively. This seems to suggest that the twin DTG peaks for the blended samples are composed of the single DTG peaks for the AW L1 and the pyrite alone, respectively. The maximum mass loss rates for the lignite and lignite-pyrite blends at the first peak occur at the same 
Chapter 5

temperature $(718 \mathrm{~K})$, however, the peak heights are different. Since the first DTG peak is related to the decomposition of lignite, the sample with a higher pyrite content has lower first DTG peak. The lower the pyrite content, the higher the peak. The first DTG peak height is directly proportional to the rate of lignite pyrolysis [58], in the case of lignite-pyrite blended samples the observed rate of pyrolysis decreases with increasing pyrite content because the pyrite in blended samples can react with organic matrix of the lignite, leading to sulphur retention in the solid.

The fact that the pyrite-free lignite does not have a secondary peak and that all the primary DTG peaks observed for the pyrite-containing samples occur at the same temperature suggest that the secondary DTG peak can only be associated with the decomposition of pyrite. Comparing the DTG peak of the pyrite mineral with the secondary peaks of the blended samples, the pyrite decomposes at a temperature about $100 \mathrm{~K}$ higher than the decomposition of pyrite in the blended samples. Pyrite in the blended samples is surrounded by organic materials of the lignite; the interaction between pyrite and the organic matrix of the lignite occurs, causing the pyrite to decompose at lower temperature. Under inert condition, pyrite decomposes to pyrrhotite during pyrolysis, according to the following reaction [108] :

$$
\mathrm{FeS}_{2}+(\mathrm{x}-2) \mathrm{H}_{2} \rightarrow \mathrm{FeS}_{\mathrm{x}}+(2-\mathrm{x}) \mathrm{H}_{2} \mathrm{~S}
$$

Pyrite-pyrrothite transformation during coal pyrolysis proceeds because of the presence of hydrogen produced from the condensation and aromatisation reactions [75, 101]. However in the presence of oxygen, pyrite will be decomposed to form hematite $\left(\mathrm{Fe}_{2} \mathrm{O}_{3}\right)$, magnetite $\left(\mathrm{Fe}_{3} \mathrm{O}_{4}\right)$, or iron sulphate $\left(\mathrm{Fe}_{2}\left(\mathrm{SO}_{4}\right)_{3}, \mathrm{FeSO}_{4}\right)$, and release sulphur dioxide $\left(\mathrm{SO}_{2}\right)$ gas [51]. 


\subsubsection{Evolution of gaseous sulphur}

To study the gases evolved during pyrolysis of lignite which contains high pyrite, 10P90L1, L2 and AW L2 samples were subjected to pyrolysis in the TGA-MS. In order to understand the effect of pyrite added to the demineralised lignite on the evolution of gaseous sulphur, sample AW L1 which is free of pyrite was also subjected to pyrolysis in the TGA-MS.

Figures 5.6 shows the evolution of hydrogen sulphide (m/z 33 and 34), while Figure 5.7 shows the evolution of sulphur dioxide gases (m/z 48 and 64$)$ during pyrolysis of AW L1, L2, AW L2 and 10P-90L1. From Figure 5.6 it can be seen that hydrogen sulphide (m/z 33 and 34) was only detected during pyrolysis of 10P-90L1 and no $\mathrm{H}_{2} \mathrm{~S}$ peaks was detected for other samples during pyrolysis. However, from Figure 5.7 it can be observed that all samples used in this experimentation released $\mathrm{SO}_{2}$ during pyrolysis.

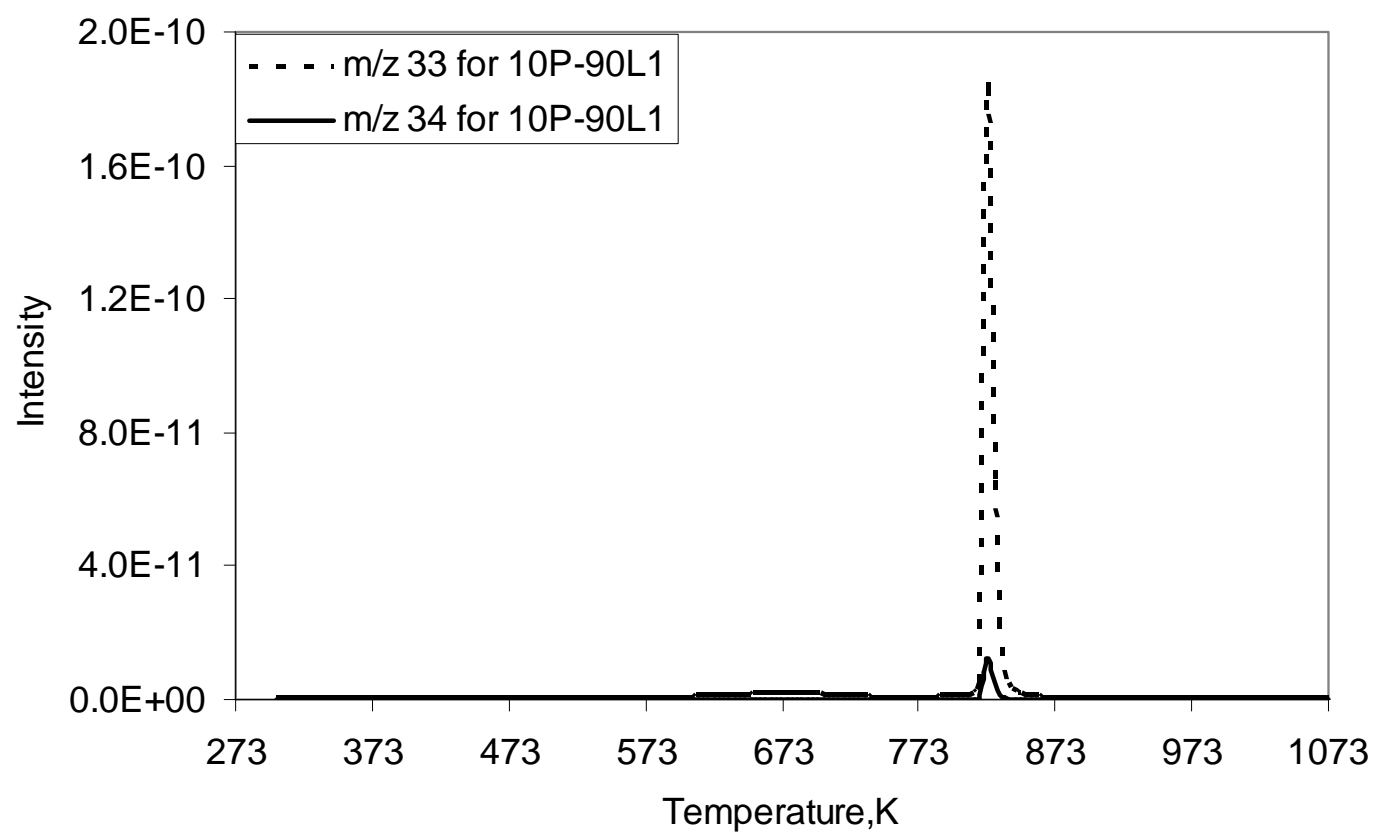

Figure 5.6. Evolution of $\mathrm{H} 2 \mathrm{~S}(\mathrm{~m} / \mathrm{z} 33$ and 34) during pyrolysis of the 10P-90L1 in TGA-MS 


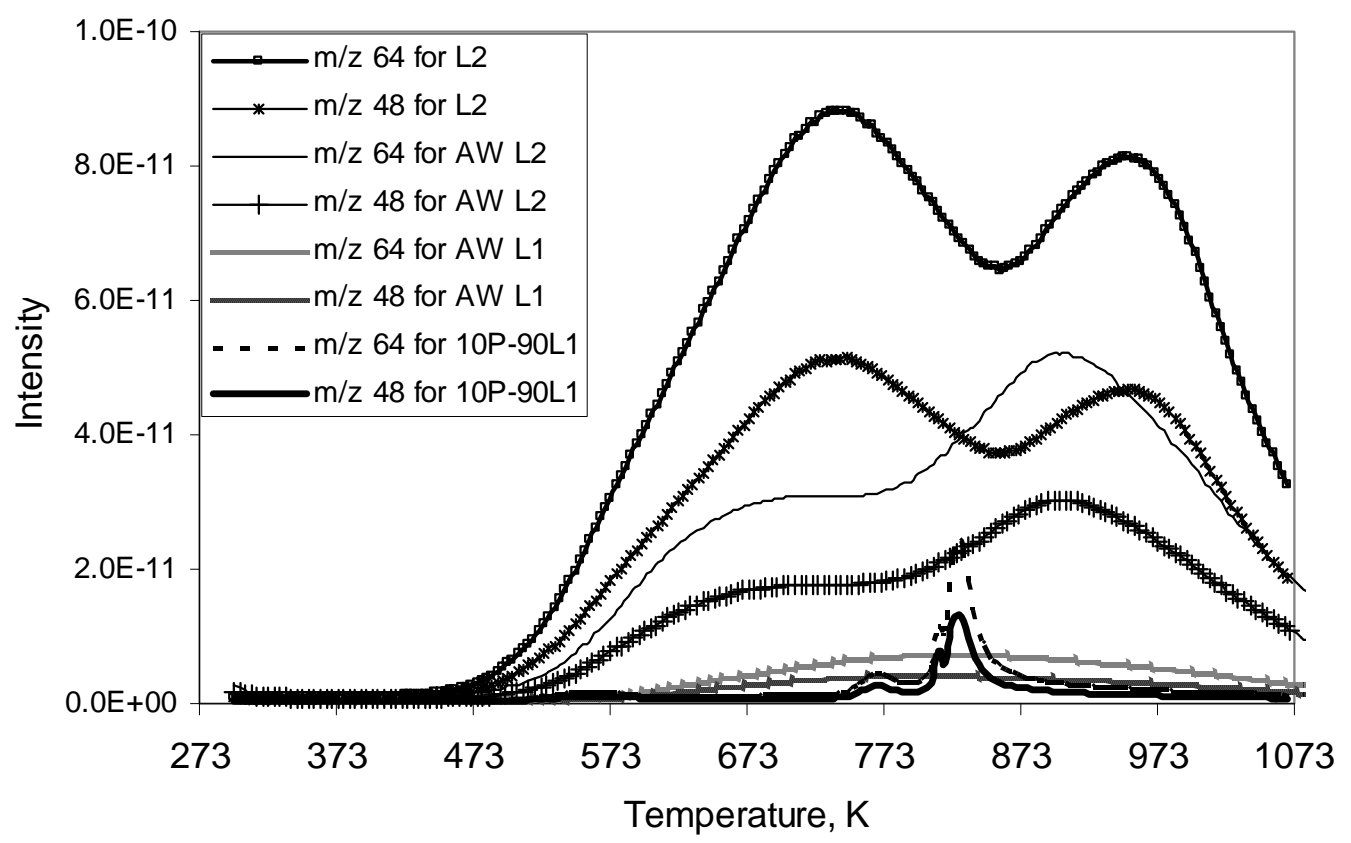

Figure 5.7.Evolution of $\mathrm{SO}_{2}(\mathrm{~m} / \mathrm{z} 48$ and 64$)$ during pyrolysis of $\mathrm{L} 2, \mathrm{AW} \mathrm{L} 2$, AW L1 and 10P-90L1 in TGA-MS

AW L1, the pyrite free sample, did not produce $\mathrm{H}_{2} \mathrm{~S}$ during pyrolysis, but small intensities of $\mathrm{SO}_{2}$ were detected (Figure 5.7). This result was supported by the fact that AW L1 contains low total sulphur with no pyrite content and has high concentration of oxygenated functional groups as shown by FTIR results in Figure 5.8. The small quantities of $\mathrm{SO}_{2}$ evolved during the pyrolysis of $\mathrm{AW} \mathrm{L1}$ is then attributed to the small amount of organic sulphur present in the lignite L1 (Table 4.3 Chapter 4), which could not be removed in the acid washing.

The high concentrations of oxygenated functional groups present in the sample L2 also affect the sulphur evolution. Although both the raw L2 and AW L2 contain high pyrite, there was no hydrogen sulphide detected during the pyrolysis of them. As can be seen from Figure 5.7, sulphur dioxide was produced in significant quantities during the pyrolysis. The pyrite in L2 and AW L2 seems to decompose to pyrrothite. The 
pyrrothite then reacts with oxygen presents in the lignite to produce $\mathrm{SO}_{2}$. The reactions can be described as follows [51] :

$$
\begin{aligned}
& \mathrm{FeS}_{2} \rightarrow \mathrm{FeS}_{x(S)}+(1-0.5 x) \mathrm{S}_{2(g)} \\
& 2 \mathrm{FeS}_{x(S)}+(1.5+2 x) \mathrm{O}_{2(g)} \rightarrow \mathrm{Fe}_{2} \mathrm{O}_{3(s)}+2 x \mathrm{SO}_{2(g)} \\
& 2 \mathrm{FeS}_{x(S)}+(3+2 x) \mathrm{O}_{2(g)} \rightarrow \mathrm{Fe}_{2}\left(\mathrm{SO}_{4}\right)_{3(s)}+(2 x-3) \mathrm{SO}_{2(g)} \\
& \mathrm{FeS}_{x(S)}+(1+x) \mathrm{O}_{2(g)} \rightarrow \mathrm{FeSO}_{4(s)}+(x-) \mathrm{SO}_{2(g)} \\
& 2 \mathrm{FeSO}_{4(s)} \leftrightarrow \mathrm{Fe}_{2} \mathrm{O}_{3(s)}+\mathrm{SO}_{3(g)}+\mathrm{SO}_{2(g)} \\
& \mathrm{Fe}_{2}\left(\mathrm{SO}_{4}\right)_{3(s)} \leftrightarrow \mathrm{Fe}_{2} \mathrm{O}_{3(s)}+3 \mathrm{SO}_{3(g)} \\
& \mathrm{SO}_{3(g)} \leftrightarrow \mathrm{SO}_{2(g)}+0.5 \mathrm{O}_{2(g)}
\end{aligned}
$$

In the previous section, it has been discussed that during coal pyrolysis, pyrite is decomposed to pyrrothite and $\mathrm{H}_{2} \mathrm{~S}$ according to reaction (R5.5) [108]. During pyrolysis of $10 \mathrm{P}-90 \mathrm{~L} 1$, the main gaseous sulphur produced is $\mathrm{H}_{2} \mathrm{~S}$ as shown in Fig 5.6. The peak of $\mathrm{H}_{2} \mathrm{~S}$ evolution of sample 10P-90L1 occurs at the same temperature as the second DTG peak discussed in section 5.2.2, therefore the second DTG peak must be associated with pyrite decomposition. The evolution of hydrogen sulphide in the blended sample is from the reaction between reactive ferrous sulphide produced from the decomposition of pyrite with the active site of lignite organic matrix. Apart from hydrogen sulphide, sulphur dioxide is also produced during this pyrolysis, although in small quantities, as can be seen from Figure 5.6. The $\mathrm{SO}_{2}$ evolution is again due to the high concentration of oxygenated functional groups present in AW L2 sample as shown by FTIR result in Figure 5.8. 


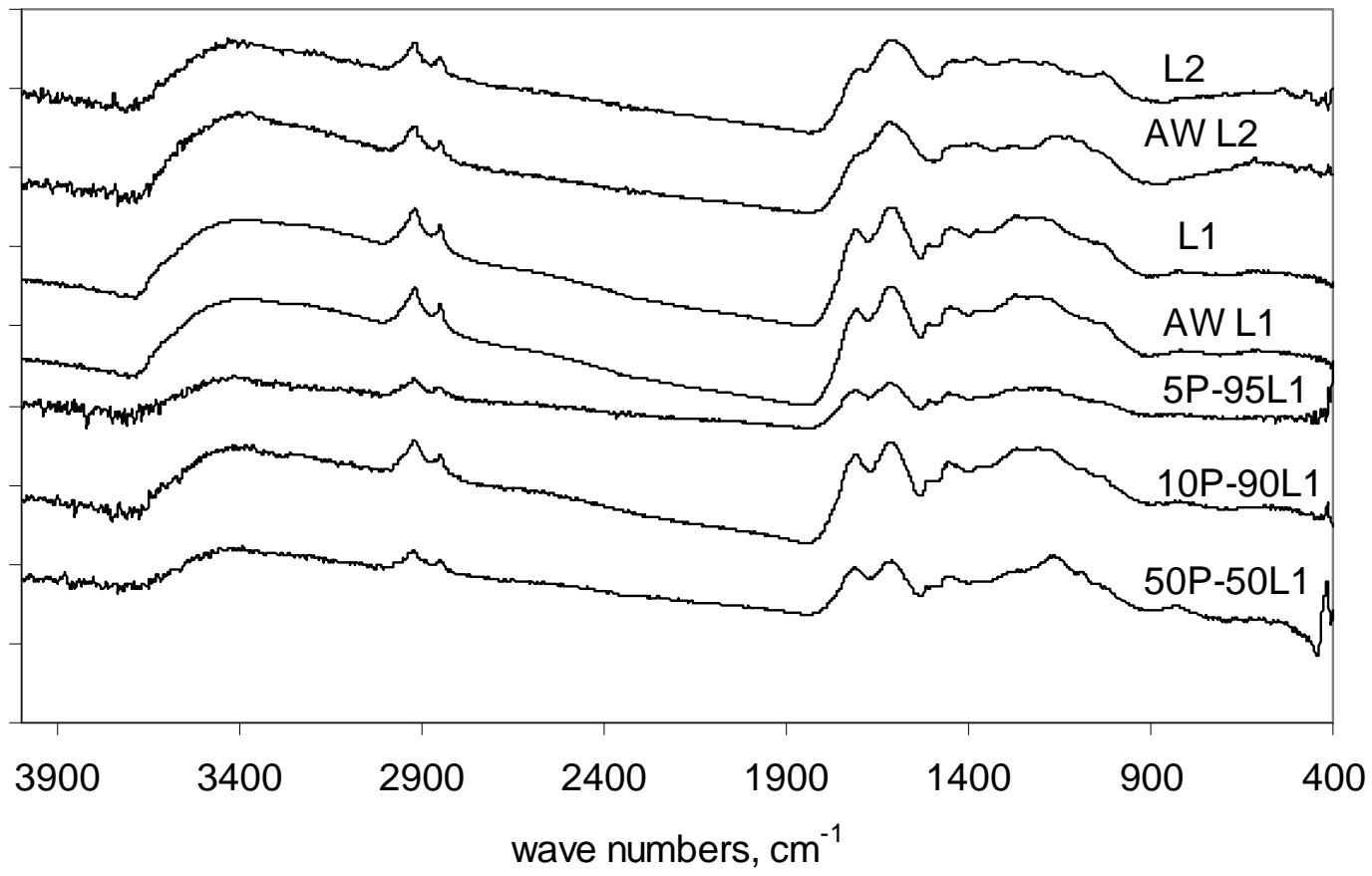

Figure 5.8. FTIR spectra of L2, AW L2, L1, AW L1, and various pyrite-lignite blends

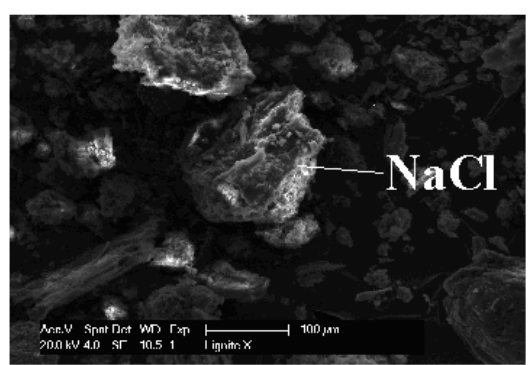

L1

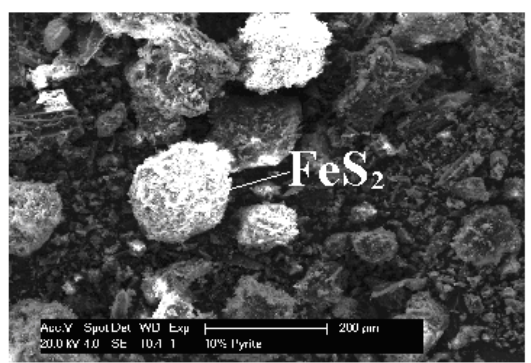

10P-90L1

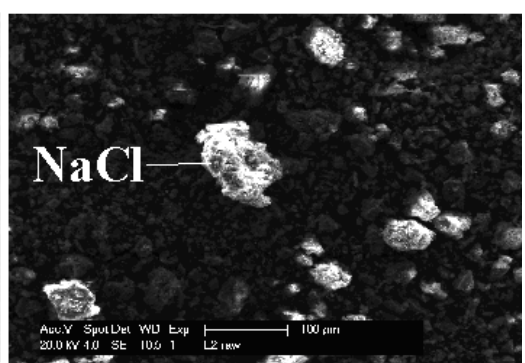

L2

Figure 5.9. SEM images of L1, 10P-90L1 and L2

It is interesting to note that L2 and AW L2 which contain high pyrite does not produce hydrogen sulphide during the pyrolysis, while 10P-90L1 sample releases significant $\mathrm{H}_{2} \mathrm{~S}$. The reason for the evolution of $\mathrm{SO}_{2}$ during pyrolysis of L2 and AW L2 samples is that the pyrite is inherently embedded in the organic matrix of the lignite. Because the organic matrix of the lignites contains highly oxygenated structure, during pyrolysis the pyrite reacts with oxygen in the lignite to produce sulphur dioxide as described in (R5.6) to (R5.12) above. On the other hand, the evolution of hydrogen sulphide during 
Chapter 5

pyrolysis of $10 \mathrm{P}-90 \mathrm{~L} 1$ is because the pyrite added to the lignite is only physically mixed with the organic matrix of the lignite. As can be seen from SEM images in Figure 5.9, pyrite is clearly seen from the bright colour of the image in 10P-90L1 sample as confirmed by EDS data collected.

\subsubsection{Sulphur forms in the chars}

The chars produced from fixed bed experiments were subjected to analyses using Carbon Sulphur (CS) analyser, SEM-EDS and FTIR to obtain information about the pyrite transformation and sulphur retention in the chars.

Figure 5.10 shows the total sulphur retention in the chars for L2, AW L2, AW L1, 5P95L1 and 10P-90L1. It can be seen that L2, the lignite with very high mineral and inorganic content as measured from the ash yield presented in Table 4.1 Chapter 4, experienced the greatest retention of total sulphur. As shown in Table 4.9 Chapter 4, L2 has extremely high $\mathrm{Na}$ and $\mathrm{Cl}$ content. During pyrolysis, inorganic matter, such as sodium, reacts with sulphur and helps to retain sulphur in the solid phase [109]. The demineralised lignite samples, AW L2 and AW L1, show significant reduction in the total sulphur retention between $573-873 \mathrm{~K}$ and above $873 \mathrm{~K}$ the retention of total sulphur did not change significantly The total sulphur retention in the blended samples decreased significantly between $673-873 \mathrm{~K}$, and the retention is almost constant above $873 \mathrm{~K}$. The higher the pyrite content, the more the total sulphur retention in the char . For $5 \mathrm{P}-95 \mathrm{~L} 1$ the total sulphur retention at $673 \mathrm{~K}$ is $90 \%$ and decreases to $49 \%$ at 1073 $\mathrm{K}$, while for $10 \mathrm{P}-90 \mathrm{~L} 1$ the total sulphur retention at $673 \mathrm{~K}$ is $93 \%$ and decreases to $52 \%$ at $1073 \mathrm{~K}$, respectively. 
Chapter 5

The retention of inorganic sulphur during pyrolysis is shown in Figure 5.11. The decomposition of inorganic sulphur in the sample with high inherent inorganic matter (L2) occurs at a slower rate than its demineralised one (AW L2); 91\% of inorganic sulphur is retained in L2 chars produced at $573 \mathrm{~K}$ and about $74 \%$ retained in the char produced at $1073 \mathrm{~K}$. The decomposition of inorganic sulphur in the demineralised samples (AW L2 and AW L1) increases significantly with increasing pyrolysis temperature. Again, it is shown in Figure 5.11 that the inherent inorganic matter present in the lignite affects the retention of inorganic sulphur in the char, the inorganic matter retains the inorganic sulphur in the solid phase [109]. The blended samples show quite similar pattern of the inorganic sulphur decomposition, 5P-95L1 and 10P-90L1 experienced significant inorganic sulphur decomposition between $673-873 \mathrm{~K}$ and became more slowly above $873 \mathrm{~K}$. FeS is formed during the lignite pyrolysis at $873 \mathrm{~K}$ as confirmed by FTIR, SEM-EDS and XRD which will be discussed further below. FeS is a stable compound; therefore after pyrite was decomposed to FeS, the inorganic sulphur decomposition in the blended samples became less significant. 


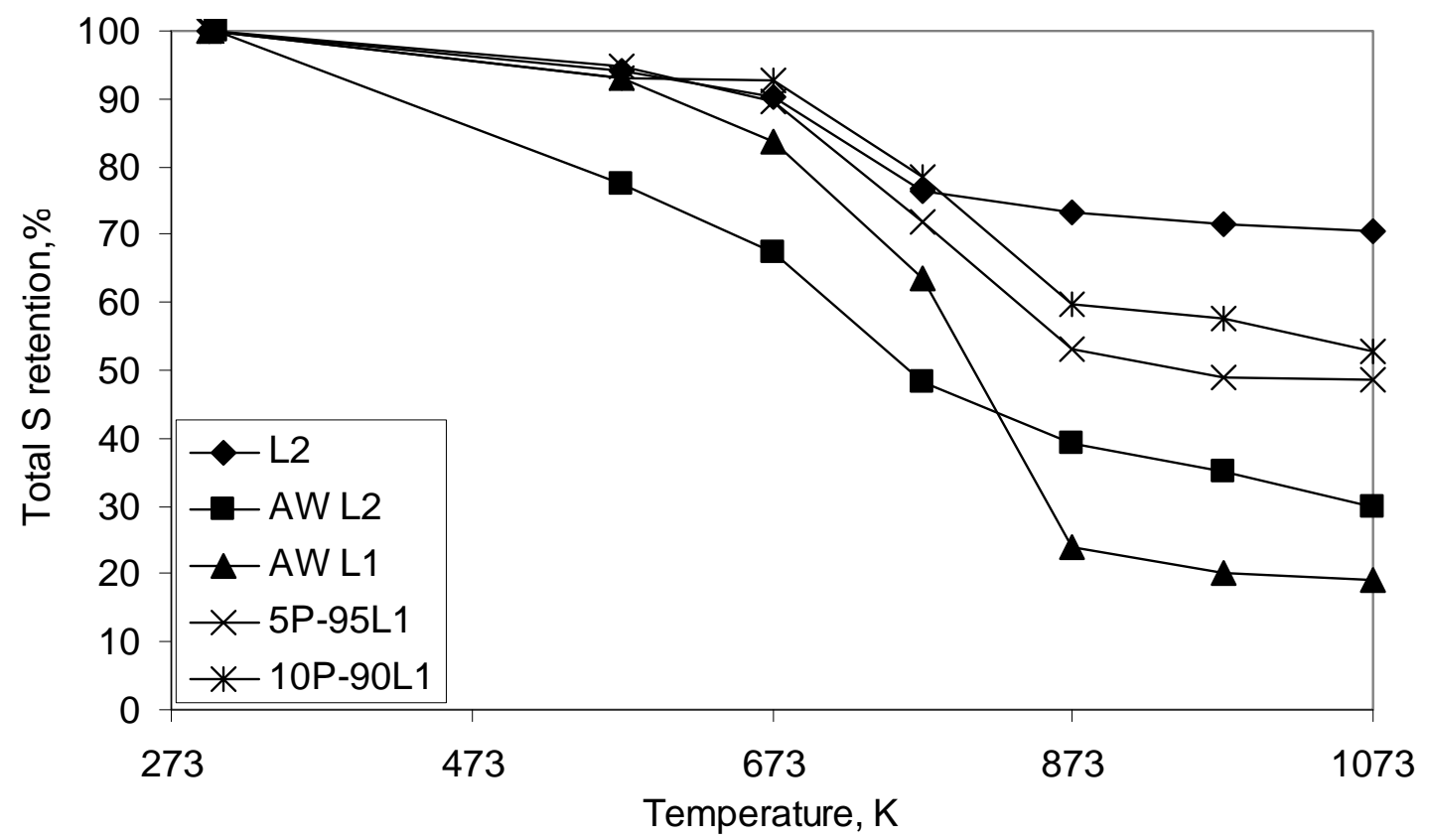

Figure 5.10. Total sulphur retention in chars produced at $573-1073 \mathrm{~K}$ during fixed bed pyrolysis

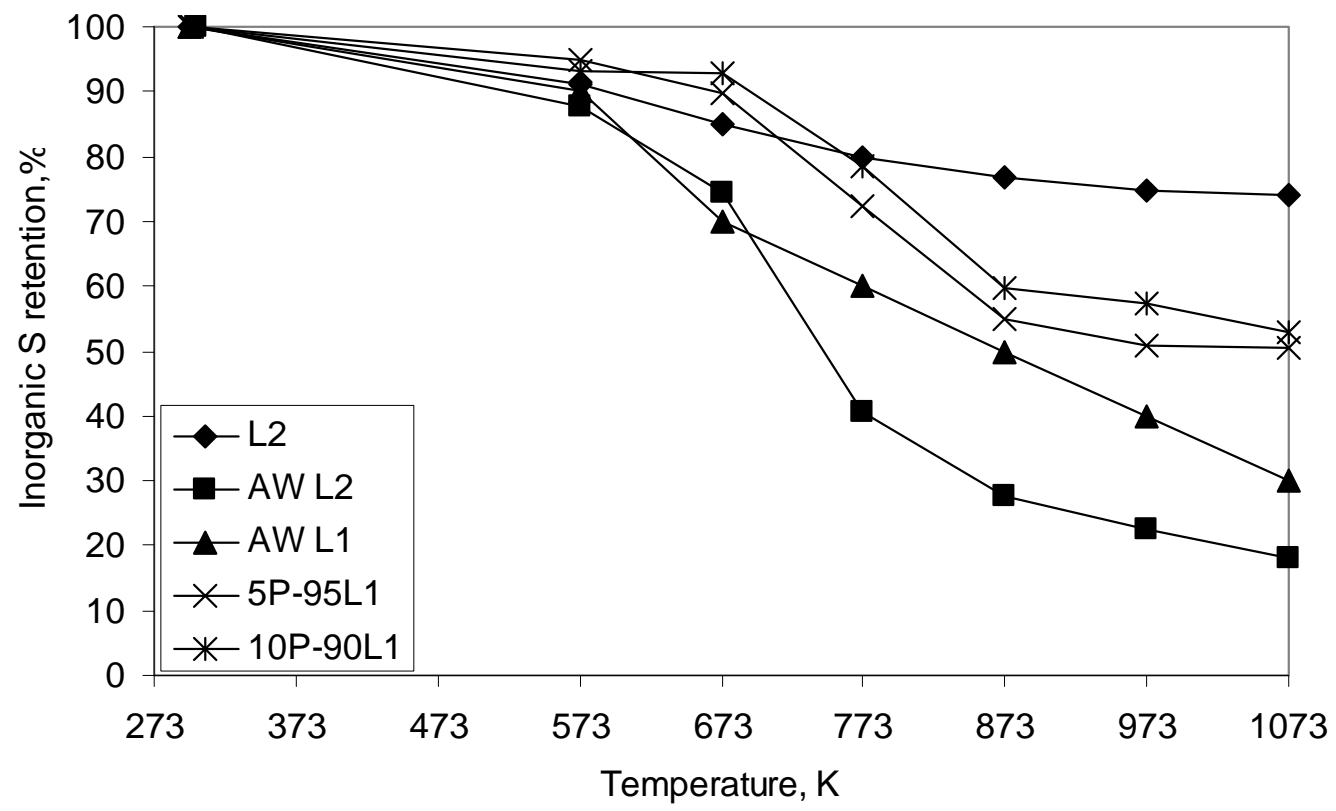

Figure 5.11. Inorganic sulphur retention of chars produced at 573-1073 K during fixed bed pyrolysis

FTIR is used to study the transformation of pyrite during lignite pyrolysis in this study. Figure 5.8 shows the FTIR spectra of the raw samples used in this research. For all samples, it can be seen that oxygenated functional groups were observed at 1000-1800 
$\mathrm{cm}^{-1}$ zone, aliphatic hydrogen stretching region at $2800-3000 \mathrm{~cm}^{-1}$ zone, aromatic structures at $700-900 \mathrm{~cm}^{-1}$ region, and hydroxyl groups at $3400-3600 \mathrm{~cm}^{-1}$ region $[17$, 18]. A great abundance of $\mathrm{C}=\mathrm{O}$ and $\mathrm{C}-\mathrm{O}-\mathrm{R}$ structures is noted by the intensity of the peaks in the $1800-1000 \mathrm{~cm}^{-1}$ region. It can be seen from Figure 5.8 that the spectra of AW L1, 5P-95L1, 10P-90L1 and 50P-50L1 are quite similar, except for mineral bands at $400-600 \mathrm{~cm}^{-1}$ and $3600-4000 \mathrm{~cm}^{-1}$. Figure 5.8 shows that the peak at $410-420 \mathrm{~cm}^{-1}$ was not observed in L1 and AW L1, however the band at $410-420 \mathrm{~cm}^{-1}$ was identified for the other raw samples. L1 and AW L1 are the pyrite-free sample, while other samples contain significant amounts of pyrite. The $410-420 \mathrm{~cm}^{-1}$ peak was attributed to pyrite [89].

A comparison of the FTIR spectra for the lignite blended with pyrite (10P-90L1) and its chars at 6 different pyrolysis temperatures is presented in Figure 5.12. In the10P-90L1 raw sample spectra, there are two distinct peaks at $2846 \mathrm{~cm}^{-1}$ and $2915 \mathrm{~cm}^{-1}$ in the aliphatic $\mathrm{C}-\mathrm{H}$ bands zone which can be attributed to symmetric and asymmetric $-\mathrm{CH}_{2}$ stretching, respectively. The intensity of these aliphatic bands decreases with increasing pyrolysis temperature. The intensity of the $\mathrm{C}-\mathrm{H}$ stretching bands disappears at the spectra of the char produced at $873 \mathrm{~K}$. The intensity of peaks of oxygenated functional groups also decreases with increasing pyrolysis temperature. The bands show very low intensity in the $873 \mathrm{~K}$ and $973 \mathrm{~K}$ chars and disappear in the $1073 \mathrm{~K}$ char. The decrease in the intensity of aliphatic and oxygenated groups is due to the loss of hydrogen and oxygen during devolatilisation $[18,87]$. As temperature increases, the oxygenated groups are decomposed, releasing mainly $\mathrm{H}_{2} \mathrm{O}$ and $\mathrm{CO}$, but also aldehydes, alcohols and acids [18]. The broad bands at $3400 \mathrm{~cm}^{-1}$ are attributed to the hydroxyl groups (-OH) due to the water content in the sample. The bands are still observable in the chars. The presence of water in the chars could be from absorption of moisture by the chars or $\mathrm{KBr}$ 
Chapter 5

during pellets preparation. By increasing pyrolysis temperature, the chars contain more carbon and less volatile matter and moisture.

The intensity of the peaks at $410-420 \mathrm{~cm}^{-1}$ decreases with increasing pyrolysis temperature. By comparing FTIR spectra of pure pyrite and pure troilite (FeS) with FTIR spectra of 10P-90L1 and its chars (Figure 12), pyrite is still present in the $773 \mathrm{~K}$ char. However, above this temperature, the pyrite band disappears from the spectra. Pyrite bands also disappear in chars produced at $873 \mathrm{~K}$ and chars of above this temperature for 5P-95L1, L2 and AW L2 samples.

SEM-EDS was used to identify the presence of pyrite in the samples as well as to study the transformation of pyrite during pyrolysis. By measuring the ratio of X-ray intensity of sulphur to iron, the form of iron sulphide in the chars produced during pyrolysis can be identified qualitatively $[60,110]$. Pyrite $\left(\mathrm{FeS}_{2}\right)$ has an $\mathrm{S} / \mathrm{Fe}$ atomic ratio of 2, pyrrothite is present when $2<\mathrm{S} / \mathrm{Fe}<1, \mathrm{~S} / \mathrm{Fe}$ atomic ratio of troilite is 1 , and elemental iron is formed when the ratio of $\mathrm{S} / \mathrm{Fe}<1.0$. From the SEM-EDS analysis, it can be confirmed that L1 is pyrite-free, no Fe was detected in the sample. 


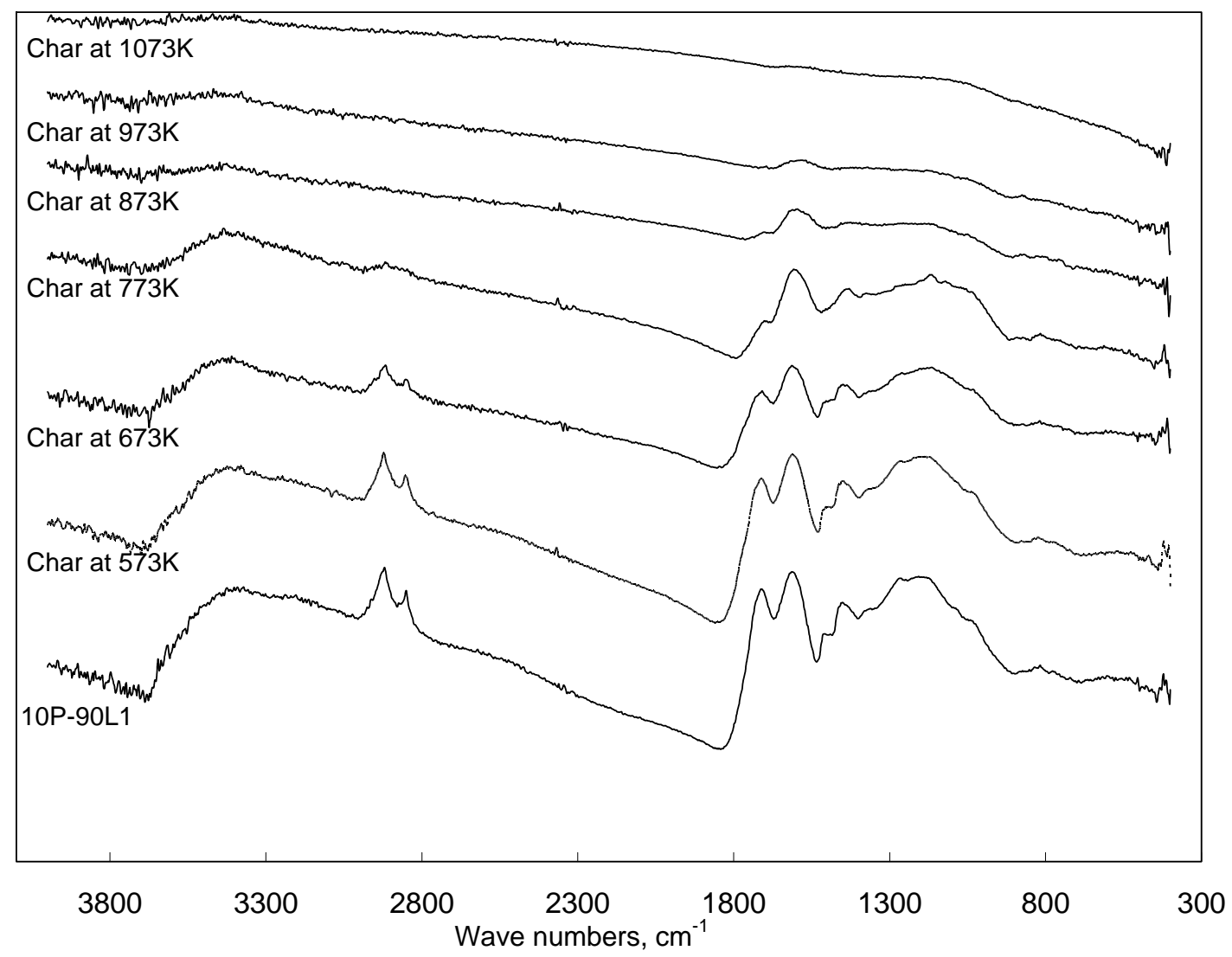

Figure 5.12. FTIR spectra of 10P-90L1 and its chars at different pyrolysis temperatures

The SEM images of the 10P-90L1 sample and its chars at $673 \mathrm{~K}, 873 \mathrm{~K}$ and $1073 \mathrm{~K}$ are shown in Figure 5.13. The SEM image of the $673 \mathrm{~K}$ char is quite similar to the SEM image of the sample before pyrolysis, but the images of chars produced at higher temperatures have different appearances. This agrees with the FTIR findings; the char produced at $673 \mathrm{~K}$ has not experienced massive structural changes yet, however by increasing the pyrolysis temperature more aliphatic and oxygenated structures are decomposed and the resulting chars have more carbon and less volatile. 


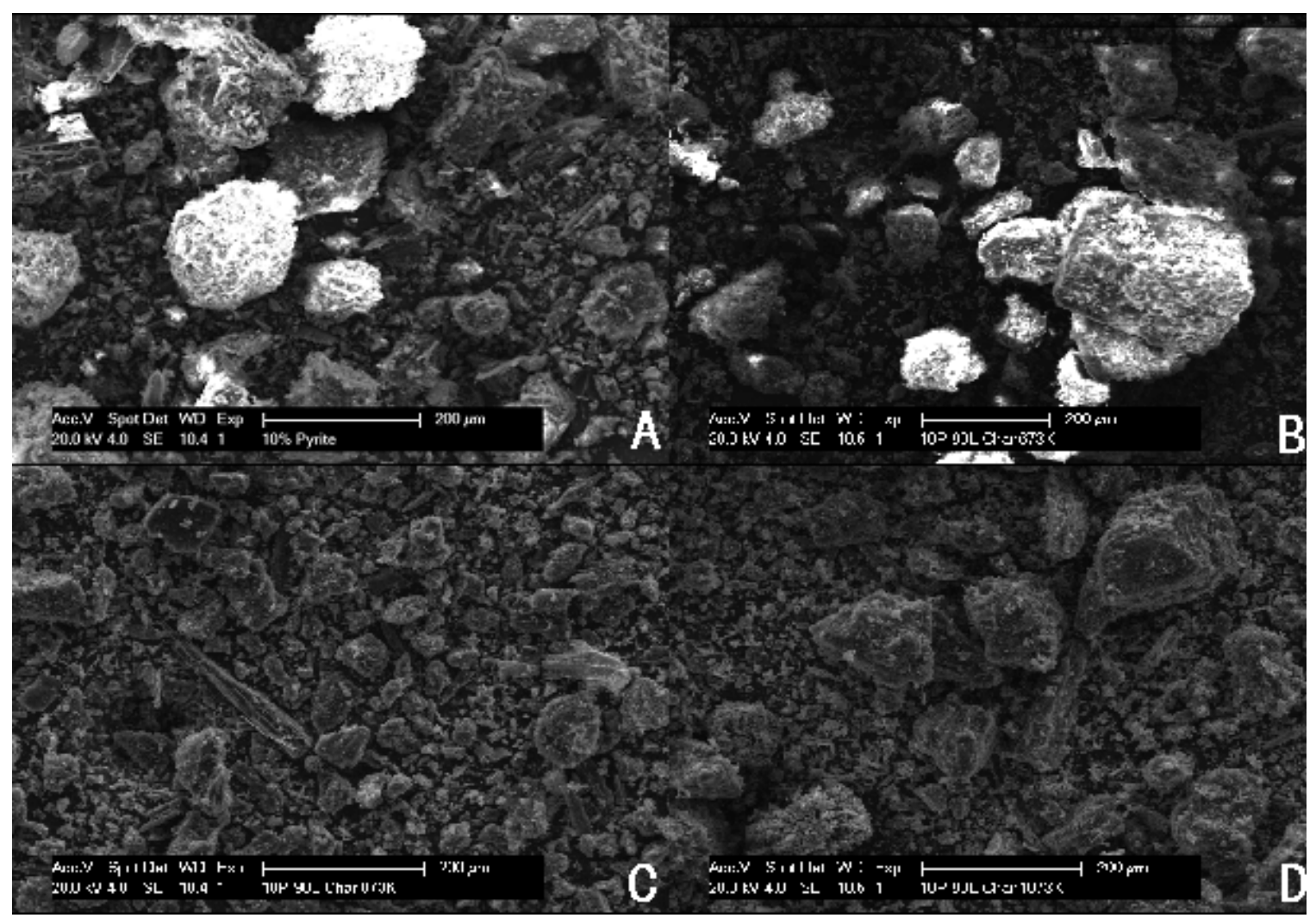

Figure 5.13. SEM images of the 10P-90L1 sample before pyrolysis (A) and its char produced at $673 \mathrm{~K}(\mathrm{~B}), 873 \mathrm{~K}(\mathrm{~A})$, and $1073 \mathrm{~K}(\mathrm{D})$, respectively.

The SEM images of the 10P-90L1 sample and its chars at $673 \mathrm{~K}, 873 \mathrm{~K}$ and $1073 \mathrm{~K}$ are shown in Figure 5.13. The SEM image of the $673 \mathrm{~K}$ char is quite similar to the SEM image of the sample before pyrolysis, but the images of chars produced at higher temperatures have different appearances. This agrees with the FTIR findings; the char produced at $673 \mathrm{~K}$ has not experienced massive structural changes yet, however by increasing the pyrolysis temperature more aliphatic and oxygenated structures are decomposed and the resulting chars have more carbon and less volatile.

By comparing the EDS spectra of samples before and after pyrolysis, the pyrite transformation can be examined. For the 5P-95L1, 10P-90L1 and L2 samples, the atomic ratio of $\mathrm{S} / \mathrm{Fe}$ is about 2 . Chars produced at $573 \mathrm{~K}$ have an $\mathrm{S} / \mathrm{Fe}$ atomic ratio very close to 2 , indicating that the pyrite has not been decomposed to any appreciable extent. Pyrite is transformed to pyrrothite at about $673 \mathrm{~K}$, because the chars produced at $673 \mathrm{~K}$ 
Chapter 5

show the atomic ratio of $\mathrm{S} / \mathrm{Fe}$ between 1 and 2 . Pyrrothite completely disappeared from the $873 \mathrm{~K}$ chars. It is shown by its EDS spectra which give an $\mathrm{S} / \mathrm{Fe}$ atomic ratio of 1 . Some of the ferrous sulphide is further decomposed to elemental iron at a very slow rate above $873 \mathrm{~K}$, it is confirmed from the EDS spectra of the chars produced at $973 \mathrm{~K}$ and $1073 \mathrm{~K}$ which gave the ratio of $\mathrm{S} / \mathrm{Fe}<1.0$.

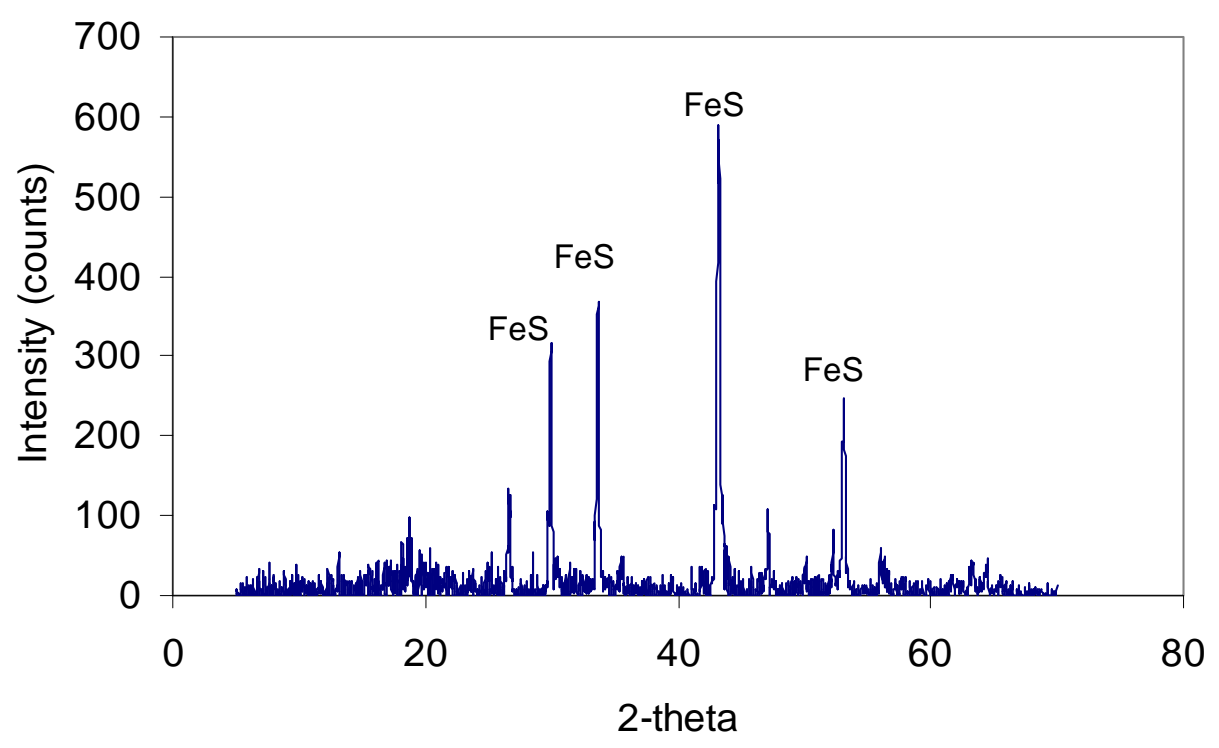

Figure 5.14. XRD spectra of 10P-90 L char produced at 873K

The transformation of $\mathrm{FeS}_{2}$ to $\mathrm{FeS}$ for chars produced at $873 \mathrm{~K}$ is also confirmed from XRD spectra applied to the chars. Figure 5.14 shows the XRD spectra of 10P-90L1 char produced at $873 \mathrm{~K}$.

The combination of analytical techniques employed to study the chars produced from lignite - pyrite blends shows that pyrite started to decompose at a temperature of $673 \mathrm{~K}$ to form pyrrothite and the pyrrothite disappeared from the samples at $873 \mathrm{~K}$ to form troilite (FeS). Since FeS is a stable compound, further decomposition of FeS to form elemental iron occurs at a slower rate during lignite pyrolysis. The chars produced at 973K and 1073K have $\mathrm{S} / \mathrm{Fe}<1.0$. 


\subsubsection{Kinetics of pyrolysis of pyrite, lignite, and lignite - pyrite blends}

Mass loss measurements performed in a thermogravimetric analyser (TGA) can be used to estimate the kinetics of coal pyrolysis [58, 104, 106, 107]. In this study, nonisothermal kinetic studies using TGA have been performed which has several advantages, including a lesser amount of data required, avoiding the overlook of the decomposition features during the entire course of pyrolysis, avoiding the variation of estimation of kinetic parameters by using single samples for the whole non-isothermal TGA range [111].

During the lignite pyrolysis, the moisture, along with some volatile matter, will be removed before $573 \mathrm{~K}$ is reached. Then at temperatures $>573 \mathrm{~K}$, the carbonisation of the complex organic matter in the lignite occurs [58]. In this investigation, kinetic parameters for lignite pyrolysis were obtained in the temperature range of 573-1003 K by assuming that the overall reaction is of the first order.

To further study the effect of pyrite during pyrolysis of lignite-pyrite blend samples, kinetic parameters for pyrite decomposition was studied in the temperature range of 773-953 K also by assuming that the reaction follows a first order reaction.

In general, the kinetic analysis of non-isothermal pyrolysis can be described as follows $[70,106]$ :

$$
\begin{aligned}
& \frac{d X}{d t}=k f(X) \\
& k=A \exp \left(\frac{-E}{R T}\right)
\end{aligned}
$$


where $X$ is the fraction decomposed, $f(X)$ is a function of the degree of reaction, $t$ is the reaction time, $\mathrm{k}$ is the rate constant, $\mathrm{A}$ is the frequency factor, $\mathrm{E}$ is the activation energy, $\mathrm{R}$ is the universal gas constant, $\mathrm{T}_{\mathrm{o}}$ is the starting temperature, $\mathrm{H}$ is the heating rate. By combining Eqs. (5.1)-(5.3), the decomposition rate can be expressed as:

$$
\frac{d X}{d t}=f(X) \frac{A}{H} \exp \left(\frac{-E}{R T}\right)
$$

Based on recent calculations, $f(X)$ is defined as $f(X)=(1-X)^{n}$, with $n$ is overall reaction order and E and A are singular values [107]. Eq. (5.4) thus can be written as:

$$
\frac{d X / d t}{(1-X)^{n}}=\frac{A}{H} \exp \left(\frac{-E}{R T}\right)
$$

Coats and Redfern [77] have integrated Eq (5.5) by expanding it into series with the boundary conditions of $\mathrm{X}=0$ for $\mathrm{T}=\mathrm{T}_{\mathrm{o}}$ and $\mathrm{X}=\mathrm{X}$ for $\mathrm{T}=\mathrm{T}$. For the case $\mathrm{n} \neq 1$ and by ignoring higher order terms of the series, the following equation was obtained :

$$
\frac{1-(1-X)^{(1-n)}}{(1-n) T^{2}}=\frac{A R}{H E}\left(1-\frac{2 R T}{E}\right) \exp \left(\frac{-E}{R T}\right)
$$

For the first order reaction ( $\mathrm{n}=1)$, Eq. (5.6) can be expressed as $[77,107]$ :

$$
\ln \left[\frac{-\ln (1-X)}{T^{2}}\right]=\ln \left\{\left(\frac{A R}{H E}\right)\left(1-\frac{2 R T}{E}\right)\right\}-\frac{E}{R T}
$$

In the analysis of TGA data, $X$ is defined in the same way with Elder and Harris [106]: 


$$
X=\frac{M_{o}-M_{t}}{M_{o}-M_{f}}
$$

where $M_{f}$ is the final mass of a TGA sample, $M_{o}$ is the initial mass and $M_{t}$ is the mass at time $\mathrm{t}$.

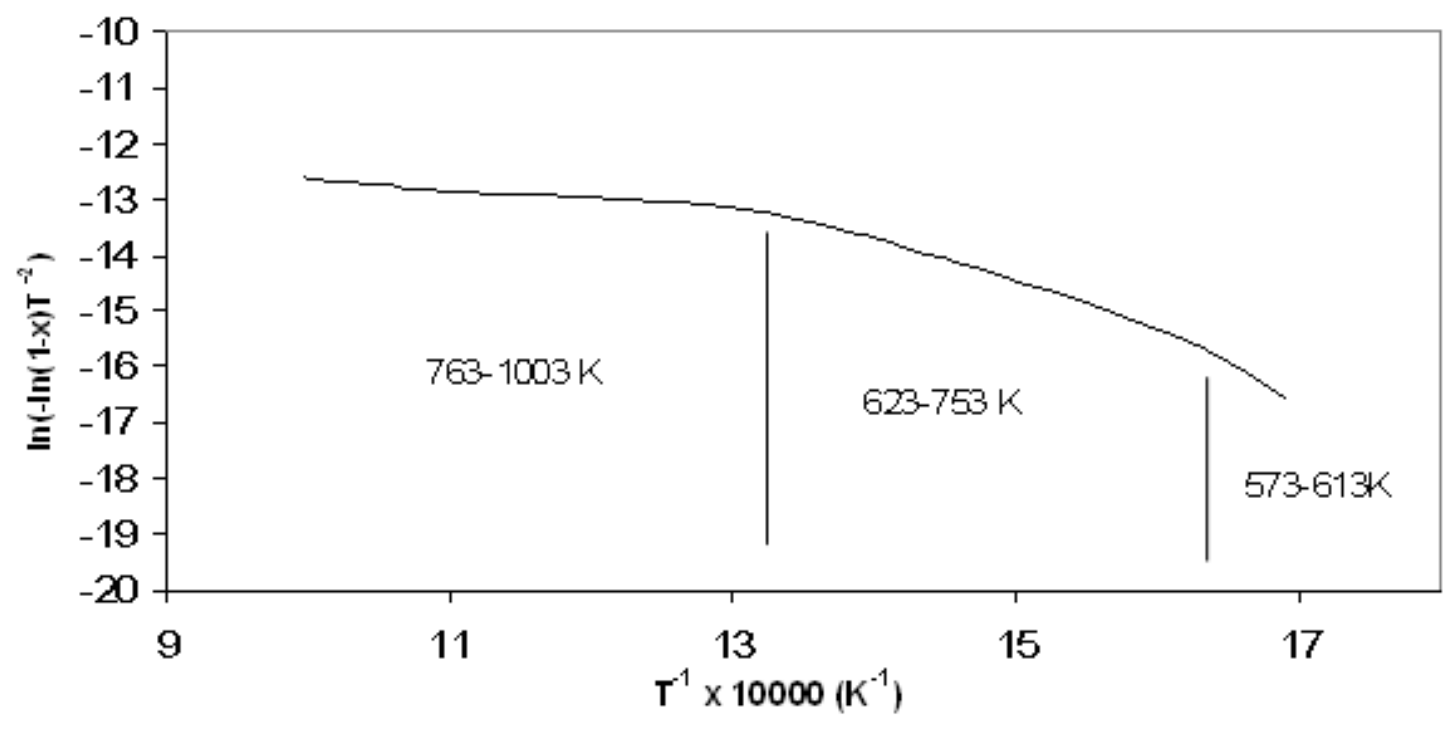

Figure 5.15. A typical plot of $\ln \left(-\ln (1-X) \cdot \mathrm{T}^{-2}\right)$ vs $\mathrm{T}^{-1}$ for lignite pyrolysis

By plotting the left-hand side of Eq. (3) versus $\mathrm{T}^{-1}$, a straight line should result if the pyrolysis follows the first order reaction. However, for lignite pyrolysis as can be seen from Figure 5.15 the plot over the whole temperature range was not a straight line but broke into three sections. Therefore, the kinetic parameters were determined over the three temperature regions, namely, 573-613K, 623-753K and 763-1003K, respectively. From the slope of each straight line section in each temperature region, the activation energy E can be determined, while A can be calculated by taking the temperature $\mathrm{T}$ at $\mathrm{W}_{\mathrm{t}}=\left(\mathrm{W}_{\mathrm{o}}-\mathrm{W}_{\mathrm{f}}\right) / 2$, where $\mathrm{W}_{\mathrm{o}}$ is the initial sample mass and $\mathrm{W}_{\mathrm{f}}$ is the final mass of the sample of each temperature region [105]. The kinetic parameters thus obtained for the lignite pyrolysis as well as pyrite decomposition are summarised in Table 5.2. 
As can be seen from Table 5.2 for the lignites and pyrite-lignite blend samples the activation energy values are found to decrease with increasing temperature over the three sections. For the lignite samples, the E values range between 107.40-147.56 $\mathrm{kJ} \mathrm{mol}^{-1}$ at $573-613 \mathrm{~K}, 43.40-64.71 \mathrm{~kJ}^{-\mathrm{mol}^{-1}}$ at $623-753 \mathrm{~K}$ and $15.86-17.0 \mathrm{~kJ}^{-\mathrm{mol}^{-1}}$ at 763-1003K, respectively. While the activation energy values for lignite blended with pyrite samples vary between $121.84-140.67 \mathrm{~kJ} \mathrm{~mol}^{-1}$ at the lowest temperature region, $55.50-63.81 \mathrm{~kJ} \mathrm{~mol}^{-1}$ at the medium temperature region, and $24.91-33.50 \mathrm{~kJ} \mathrm{~mol}^{-1}$ at the highest temperature region.

The E value for samples with higher pyrite or mineral contents should be higher, as the decomposition of the mineral contents needs higher energy [3]. However, the activation energy values of all samples at the two lower temperature ranges of 573-613 K and 623$753 \mathrm{~K}$ as stated in the previous paragraph do not vary significantly. In the highest temperature range, the activation energy of demineralised samples is lower than their original samples. For the pyrite-lignite blends, the E values increases with increasing pyrite content in the highest temperature range (763-1003 K). It is in the temperature range of $763-1003 \mathrm{~K}$ when the pyrite to pyrrothite transformation occurs. In this study the activation energy for decomposition of the pyrite mineral between 773 and $953 \mathrm{~K}$ is $121.60 \mathrm{~kJ} \cdot \mathrm{mol}^{-1}$. Previous studies on the pyrite decomposition under nitrogen atmosphere showed that the apparent activation energy obtained was in the range of 96$113 \mathrm{~kJ} \mathrm{~mol}^{-1}$ [57]. The increase in the E value with increasing pyrite content in the 763$1003 \mathrm{~K}$ temperature range is due to higher activation energy needed to decompose pyrite in the sample.

The frequency factors of pyrolysis of the lignites and the lignite - pyrite blends decrease with increasing temperature over the three temperature regions. For lignites the frequency factors are between $1.5 \times 10^{8}-4.9 \times 10^{11} \mathrm{~min}^{-1}$ at $573-613 \mathrm{~K}$, between $6.2 \times 10^{2}$ - 
Chapter 5

$2.6 \times 10^{4} \mathrm{~min}^{-1}$ at $623-753 \mathrm{~K}$ and $17.0-22.7 \mathrm{~min}^{-1}$ at $763-1003 \mathrm{~K}$, respectively. While the A values for pyrite-lignite blends range between $3.0 \times 10^{9}-1.4 \times 10^{11} \mathrm{~min}^{-1}$ at $573-613 \mathrm{~K}$, $3.5 \times 10^{3}-2.4 \times 10^{4} \min ^{-1}$ at $623-753 \mathrm{~K}$, and $35.7-74.4 \min ^{-1}$ at $763-1003 \mathrm{~K}$, respectively. A for mineral pyrite decomposition at $773-953 \mathrm{~K}$ is $5.2 \times 10^{6} \mathrm{~min}^{-1}$. From Table 5.2, it can be seen that in the highest temperature region for the pyrite-lignite blended samples, A value increases with increasing pyrite content. The reaction rates of the pyrolysis of the lignites, pyrite-lignite blends and the pyrite mineral are also summarised in Table 5.2. 
Table 5.2. Kinetic parameters of pyrolysis of lignites, pyrite and lignite-pyrite blends

\begin{tabular}{|c|c|c|c|c|c|}
\hline Sample & Temperature,K & $\mathrm{E}, \mathrm{kJ} . \mathrm{mol}^{-1}$ & $\mathrm{~A}, \min ^{-1}$ & $\mathrm{R}^{2}$ & Reaction rate \\
\hline L1 & $\begin{array}{l}573-613 \\
623-753 \\
763-1003\end{array}$ & $\begin{array}{l}147.56 \\
64.71 \\
17.0\end{array}$ & $\begin{array}{l}4.9 \times 10^{11} \\
2.6 \times 10^{4} \\
18.4\end{array}$ & $\begin{array}{l}0.98 \\
0.98 \\
0.93\end{array}$ & $\begin{array}{l}\mathrm{dX} / \mathrm{dt}=4.9 \times 10^{11} \exp (-17748 / \mathrm{T})(1-\mathrm{X}) \\
\mathrm{dX} / \mathrm{dt}=2.6 \times 10^{4} \exp (-7783 / \mathrm{T})(1-\mathrm{X}) \\
\mathrm{dX} / \mathrm{dt}=18.4 \exp (-2041 / \mathrm{T})(1-\mathrm{X})\end{array}$ \\
\hline AW L1 & $\begin{array}{l}573-613 \\
623-753 \\
763-1003\end{array}$ & $\begin{array}{l}107.40 \\
59.73 \\
16.74\end{array}$ & $\begin{array}{l}1.5 \times 10^{8} \\
1.4 \times 10^{4} \\
17.2\end{array}$ & $\begin{array}{l}0.98 \\
0.99 \\
0.93\end{array}$ & $\begin{array}{l}\mathrm{dX} / \mathrm{dt}=1.5 \times 10^{8} \exp (-12917 / \mathrm{T})(1-\mathrm{X}) \\
\mathrm{dX} / \mathrm{dt}=1.4 \times 10^{4} \exp (-7184 / \mathrm{T})(1-\mathrm{X}) \\
\mathrm{dX} / \mathrm{dt}=19.7 \exp (-2013 / \mathrm{T})(1-\mathrm{X})\end{array}$ \\
\hline 5P-95L1 & $\begin{array}{l}573-613 \\
623-753 \\
763-1003\end{array}$ & $\begin{array}{l}140.67 \\
63.81 \\
24.91\end{array}$ & $\begin{array}{l}1.4 \times 10^{11} \\
2.4 \times 10^{4} \\
35.7\end{array}$ & $\begin{array}{l}0.95 \\
0.99 \\
0.95\end{array}$ & $\begin{array}{l}\mathrm{dX} / \mathrm{dt}=1.4 \times 10^{11} \exp (-16919 / \mathrm{T})(1-\mathrm{X}) \\
\mathrm{dX} / \mathrm{dt}=2.4 \times 10^{4} \exp (7675 / \mathrm{T})(1-\mathrm{X}) \\
\mathrm{dX} / \mathrm{dt}=35.7 \exp (-2996 / \mathrm{T})(1-\mathrm{X})\end{array}$ \\
\hline 10P-90L1 & $\begin{array}{l}573-613 \\
623-753 \\
763-1003\end{array}$ & $\begin{array}{l}121.84 \\
62.33 \\
28.30\end{array}$ & $\begin{array}{l}3.0 \times 10^{9} \\
1.8 \times 10^{4} \\
54.2\end{array}$ & $\begin{array}{l}0.98 \\
0.99 \\
0.95\end{array}$ & $\begin{array}{l}\mathrm{dX} / \mathrm{dt}=3.0 \times 10^{9} \exp (-14654 / \mathrm{T})(1-\mathrm{X}) \\
\mathrm{dX} / \mathrm{dt}=1.8 \times 10^{4} \exp (-7496 / \mathrm{T})(1-\mathrm{X}) \\
\mathrm{dX} / \mathrm{dt}=54.2 \exp (-3404 / \mathrm{T})(1-\mathrm{X})\end{array}$ \\
\hline 50P-50L1 & $\begin{array}{l}573-613 \\
623-753 \\
763-1003\end{array}$ & $\begin{array}{l}124.14 \\
55.50 \\
33.5\end{array}$ & $\begin{array}{l}3.8 \times 10^{9} \\
3.5 \times 10^{3} \\
74.4\end{array}$ & $\begin{array}{l}0.98 \\
0.99 \\
0.97\end{array}$ & $\begin{array}{l}\mathrm{dX} / \mathrm{dt}=3.8 \times 10^{9} \exp (-14931 / \mathrm{T})(1-\mathrm{X}) \\
\mathrm{dX} / \mathrm{dt}=3.5 \times 10^{3} \exp (-6675 / \mathrm{T})(1-\mathrm{X}) \\
\mathrm{dX} / \mathrm{dt}=74.4 \exp (-4029 / \mathrm{T})(1-\mathrm{X})\end{array}$ \\
\hline Pyrite & $773-953$ & 121.60 & $5.2 \times 10^{6}$ & 0.92 & $\mathrm{dX} / \mathrm{dt}=5.2 \times 10^{6} \exp (-14625 / \mathrm{T})(1-\mathrm{X})$ \\
\hline AW L2 & $\begin{array}{l}573-613 \\
623-753 \\
763-1003\end{array}$ & $\begin{array}{l}126.48 \\
50.97 \\
15.86\end{array}$ & $\begin{array}{l}1.0 \times 10^{10} \\
2.5 \times 10^{3} \\
22.7\end{array}$ & $\begin{array}{l}0.99 \\
0.99 \\
0.94\end{array}$ & $\begin{array}{l}\mathrm{dX} / \mathrm{dt}=1.0 \times 10^{10} \exp (-15212 / \mathrm{T})(1-\mathrm{X}) \\
\mathrm{dX} / \mathrm{dt}=2.5 \times 10^{3} \exp (-6130 / \mathrm{T})(1-\mathrm{X}) \\
\mathrm{dX} / \mathrm{dt}=22.7 \exp (-1907 / \mathrm{T})(1-\mathrm{X})\end{array}$ \\
\hline L2 & $\begin{array}{l}573-613 \\
623-753 \\
763-1003\end{array}$ & $\begin{array}{l}126.94 \\
43.40 \\
16.34\end{array}$ & $\begin{array}{l}1.4 \times 10^{10} \\
6.2 \times 10^{2} \\
21.7\end{array}$ & $\begin{array}{l}0.96 \\
0.99 \\
0.90\end{array}$ & $\begin{array}{l}\mathrm{dX} / \mathrm{dt}=1.4 \times 10^{10} \exp (-15268 / \mathrm{T})(1-\mathrm{X}) \\
\mathrm{dX} / \mathrm{dt}=6.2 \times 10^{2} \exp (-5220 / \mathrm{T})(1-\mathrm{X}) \\
\mathrm{dX} / \mathrm{dt}=17 \exp (-1964.8 / \mathrm{T})(1-\mathrm{X})\end{array}$ \\
\hline
\end{tabular}




\subsection{Summary}

The TGA pyrolysis experiments confirmed that during pyrolysis of pyrite alone in nitrogen the pyrite $(\mathrm{S} / \mathrm{Fe}=2)$ transforms to pyrrothite $(1<\mathrm{S} / \mathrm{Fe}<2)$ at ca $720 \mathrm{~K}$ and then to troilite $(\mathrm{S} / \mathrm{Fe}=1)$ at $1200 \mathrm{~K}$. Some of the troilite is further decomposed to elemental iron $(\mathrm{S} / \mathrm{Fe}<1)$ and gaseous elemental sulphur above $1200 \mathrm{~K}$.

Twin DTG peaks were observed during pyrolysis of pyrite-lignite blended samples. The first peak was attributed to the devolatilisation of the lignite, while the second peak was related to the pyrite decomposition. By comparing the DTG peaks of pyrite decomposition of blended samples with pyrite mineral, it was observed that pyrite decomposition in the pyrite-lignite blends occurs at temperature about $100 \mathrm{~K}$ lower than the pyrite mineral alone.

Hydrogen sulphide $\left(\mathrm{H}_{2} \mathrm{~S}\right)$ is the major sulphurous gas product released during the pyrolysis of the pyrite-lignite blends, with only a small quantity of sulphur dioxide $\left(\mathrm{SO}_{2}\right)$ as the co-product. However, the inherent pyrite in the lignite with high pyrite content reacts with the oxygenate groups in the lignite to release $\mathrm{SO}_{2}$ as the dominant sulphurous gas product and no hydrogen sulphide during pyrolysis.

Inherent inorganic matter in the lignite helps retaining part of the sulphur in the char. The retention of inorganic sulphur in the blended samples was almost constant above $873 \mathrm{~K}$ following complete transformation of pyrite to troilite as confirmed by FTIR, SEM-EDS and XRD analysis.

Kinetic parameters were obtained for the lignites, the lignite - pyrite blends and the pyrite, assuming the first-order reactions. The activation energy (E) and frequency factor (A) of the pyrolysis of the lignite samples decrease with increasing temperature 
over the three temperature regions; at 573-613K E ranges between 107.40147.56kJ.mol ${ }^{-1}$ and A ranges between $1.5 \times 10^{8}-4.9 \times 10^{11} \mathrm{~min}^{-1}$, at $623-753 \mathrm{~K} \mathrm{E}$ ranges

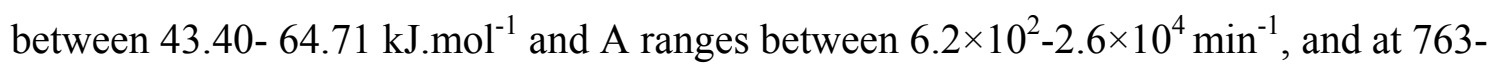
1003K E ranges between 15.86-17.0 $\mathrm{kJ}^{\mathrm{m}} \mathrm{mol}^{-1}$ and A ranges between 17.0-22.7 $\mathrm{min}^{-1}$, respectively. For the blended samples, the $\mathrm{E}$ and $\mathrm{A}$ values also decrease with increasing temperature, at 573-613K E ranges between 121.84-140.67 $\mathrm{kJ}^{\mathrm{m}} \mathrm{mol}^{-1}$ and A ranges between $3.0 \times 10^{9}-1.4 \times 10^{11} \mathrm{~min}^{-1}$, at $623-753 \mathrm{~K}$ E ranges between $55.50-63.81 \mathrm{~kJ}^{-\mathrm{mol}^{-1}}$ and A ranges between $3.5 \times 10^{3}-2.4 \times 10^{4} \mathrm{~min}^{-1}$, and at $763-1003 \mathrm{~K} \mathrm{E}$ ranges between 24.91-33.50 kJ.mol ${ }^{-1}$ and A ranges between 35.7-74.4 $\mathrm{min}^{-1}$, respectively. $\mathrm{E}$ and $\mathrm{A}$ obtained for pyrite mineral decomposition between $773-953 \mathrm{~K}$ is $121.60 \mathrm{~kJ}^{\mathrm{mol}}{ }^{-1}$ and $5.2 \times 10^{6} \mathrm{~min}^{-1}$, respectively. These high $\mathrm{E}$ and $\mathrm{A}$ values are the cause of increasing $\mathrm{E}$ and $\mathrm{A}$ values in the highest temperature region with increasing pyrite content in the blended samples.

The transformations of pyrite mineral, inherent pyrite in lignite and pyrite-lignite blends have been discussed in this chapter. The interactions between pyrite with organic matrix of lignite which contains high concentration of oxygenated structures as well as with inorganic matter presents in the lignite have been understood. The next chapter, Chapter 6 , will be devoted to studying the transformation of sulphate during lignite pyrolysis. 


\section{CHAPTER 6}

\section{Sulphate Transformation during Lignite Pyrolysis}

\section{1. Introduction}

The amount of sulphate sulphur in freshly mined coals is usually low, around $0.1 \%$ and sometimes negligible. However, in weathered coals the sulphate sulphur content can be quite significant [4]. In some coals with low pyrite and high organic sulphur, the organic sulphur can oxidise to form calcium sulphate $\left(\mathrm{CaSO}_{4}\right) \cdot \mathrm{CaSO}_{4}$ may also be introduced into a coal deposit by ground water [37]. In coals with high pyrite, the pyrite is easily oxidised to form iron sulphates $\left(\mathrm{FeSO}_{4}\right.$ or $\left.\mathrm{Fe}_{2}\left(\mathrm{SO}_{4}\right)_{3}\right)$ during coal stockpiling [110]. Zinc sulphate $\left(\mathrm{ZnSO}_{4}\right)$ is also present in some coals [38]. Since sulphate sulphur does not present significantly in fresh coals, there have been few studies about sulphate transformation during coal pyrolysis in the literature.

Generally, thermal degradation of sulphate in inert atmosphere can be described as follows [59]:

$\mathrm{MeSO}_{4(\mathrm{~s})} \leftrightarrow \mathrm{MeO}_{(\mathrm{s})}+\mathrm{SO}_{3(\mathrm{~g})}$

$\mathrm{SO}_{3(\mathrm{~g})} \leftrightarrow \mathrm{SO}_{2(\mathrm{~g})}+1 / 2 \mathrm{O}_{2(\mathrm{~g})}$

where Me is the inherent metal in coal.

Medvedev and Petropolskaya [112] studied the decomposition of sulphates and sulphate additives in coal during pyrolysis. They found that the thermal decomposition of sulphates is lower when surrounded by carbon rather than in their pure compounds. 
Chapter 6

Mullens et al [38] studied thermal decomposition of sulphate model compounds using atmospheric-pressure temperature-programmed pyrolysis (AP TPR) coupled to a mass spectrometer (MS). The compounds used in their study were calcium sulphate, zinc sulphate, iron (II) sulphate (ferrous sulphate) and iron (III) sulphate (ferric sulphate). The decomposition of the sulphates under inert atmosphere follows reactions (R1) and (R2) producing $\mathrm{SO}_{2}$ as the sulphur gas. It has been found that $\mathrm{CaSO}_{4}$ is a very stable compound. In an inert atmosphere $\mathrm{CaSO}_{4}$ decomposes above $1273 \mathrm{~K}, \mathrm{ZnSO}_{4}$ at $843 \mathrm{~K}$ and both iron sulphates at $743 \mathrm{~K}$.

In the presence of organic matter, the decomposition of sulphates occur at lower temperatures[9, 64]. Yan et al [9] studied the thermal decomposition of $\mathrm{Fe}_{2}\left(\mathrm{SO}_{4}\right)_{3}$ and $\mathrm{ZnSO}_{4}$ impregnated on the char under helium atmosphere. $\mathrm{SO}_{2}$ is the only gaseous sulphur evolved during thermal decomposition of the two compounds. $\mathrm{Fe}_{2}\left(\mathrm{SO}_{4}\right)_{3}$ decomposes at $473 \mathrm{~K}$ and $\mathrm{ZnSO}_{4}$, at $603 \mathrm{~K}$.

Ibara et al [60] reported that iron sulphates present in weathered coals decompose at 573 $\mathrm{K}$ during pyrolysis. $\mathrm{CaSO}_{4}$ in the coals starts to decompose at $623 \mathrm{~K}$. They also observed an increase in organic sulphur in char during pyrolysis while the iron sulphate decomposition occurs. This suggests the incorporation of the released sulphur into char as organic sulphur. These researchers also noted that iron sulphates may be converted to complex sulphide which is not determinable by chemical analysis, therefore giving inaccuracy in the determination of organic sulphur in the char. The temperatures of decomposition of typical pure sulphates alone and sulphates in coal are summarised in Table $1[9,38,60,110,112-114]$.

The amount of volatile sulphur released from the decomposition of a sulphate depends on the volatility of the compound $[61,112]$. A greater amount of $\mathrm{FeSO}_{4}$ can be 
Chapter 6

converted to the gas phase sulphur during thermal decomposition than $\mathrm{CaSO}_{4}$. Therefore, the residual sulphate found in the char is usually in the form of calcium sulphate.

Table 6.1 Comparison of decomposition temperatures of selected sulphates $[9,38,60,110,112-114]$

\begin{tabular}{|l|l|l|}
\hline Sulphate compounds & $\begin{array}{l}\text { Temperature of decomposition, } \\
\text { pure sulphate }(\mathrm{K})\end{array}$ & $\begin{array}{l}\text { Temperature of } \\
\text { decomposition, sulphate in } \\
\text { coal }(\mathrm{K})\end{array}$ \\
\hline $\mathrm{BaSO}_{4}$ & $1373-1423$ & na \\
\hline $\mathrm{CaSO}_{4}$ & $>1273 \mathrm{~K}$ & $573-623$ \\
\hline $\mathrm{FeSO}_{4}$ & $723-753$ & 573 \\
\hline $\mathrm{Fe}_{2}\left(\mathrm{SO}_{4}\right)_{3}$ & $723-753$ & $473-573$ \\
\hline $\mathrm{FeSO}_{4} \cdot \mathrm{Na}_{2} \mathrm{SO}_{4}$ & $723-753$ & 523 \\
\hline $\mathrm{MgSO}_{4}$ & $>1200$ & na \\
\hline $\mathrm{MnSO}_{4}$ & ca $973 \mathrm{~K}$ & na \\
\hline $\mathrm{SnSO}_{4}$ & $>651$ & na \\
\hline $\mathrm{Ti}_{\left(\mathrm{SO}_{4}\right)_{2}}$ & $423-873$ & na \\
\hline $\mathrm{V}_{2}\left(\mathrm{SO}_{4}\right)_{3}$ & $>653$ & na \\
\hline $\mathrm{ZnSO}$ & 603 \\
\hline $\mathrm{Zr}\left(\mathrm{SO}_{4}\right)_{2}$ & 843 & na \\
\hline
\end{tabular}

In this study, three sulphates commonly found in coal, namely calcium sulphate $\left(\mathrm{CaSO}_{4}\right)$ and ferrous sulphate $\left(\mathrm{FeSO}_{4} \cdot 7 \mathrm{H}_{2} \mathrm{O}\right)$ and ferric sulphate $\left(\mathrm{Fe}_{2}\left(\mathrm{SO}_{4}\right)_{3} .9 \mathrm{H}_{2} \mathrm{O}\right)$ were studied in three different atmospheres, namely, nitrogen, a mixture of $50 \%$ nitrogen and $50 \% \mathrm{CO}_{2}\left(\mathrm{~N}_{2} / \mathrm{CO}_{2}\right.$ mixture) and air, in a thermogravimetric analyser (TGA) at a constant heating rate of $10 \mathrm{~K} \cdot \mathrm{min}^{-1}$. The behaviour of sulphates during pyrolysis of sulphates impregnated in low-sulphur lignite as well as a fresh lignite with high sulphate sulphur were also pyrolysed in a TGA, a TGA coupled with a mass spectrometer (TGAMS) and a fixed bed reactor. Several complimentary analyses, namely, SEM-EDS, 
Chapter 6

XRD, FTIR and a carbon sulphur analyser were also employed to study the transformation of sulphate during lignite pyrolysis.

Large lignite reserves in Australia are an important source of energy. With its high sulphur content, the sulphate may present during the storage and processing. Some of lignites with high sulphur and inorganic matter cannot be utilised in a conventional high temperature combustion or gasification since the inorganic matter is directly related to cause slagging, fouling and corrosion [115]. In the fluidised bed combustors previous studies showed that sodium, calcium and sulphate rich components form a low melting eutectic which leads to bed material agglomeration, bed defluidisation and ash deposition $[10,116,117]$. Therefore a better understanding of sulphate transformation during lignite pyrolysis helps devise appropriate sulphur capture strategies.

\section{2 Results and discussion}

\subsubsection{Calcium sulphate transformation}

Pure calcium sulphate thermal decomposition was studied in a TGA under three different gas atmospheres, namely, nitrogen, the $\mathrm{N}_{2} / \mathrm{CO}_{2}$ mixture and air. The results are shown in Figure 6.1. It can be seen that $\mathrm{CaSO}_{4}$ thermal decomposition showed a quite similar behaviour in the nitrogen and $\mathrm{N}_{2} / \mathrm{CO}_{2}$ mixture atmospheres. In those two atmospheres, $\mathrm{CaSO} 4$ is very stable until approximately $1400 \mathrm{~K}$. $\mathrm{CaSO}_{4}$ decomposition occurs between 1400 and $1700 \mathrm{~K}$ and above $1700 \mathrm{~K}$ the remaining mass is almost the same since the decomposition has completed. While in air $\mathrm{CaSO}_{4}$ decomposes at temperatures between 1500-1700 K. The decomposition of $\mathrm{CaSO}_{4}$ can be written as follows: 


$$
\begin{aligned}
& \mathrm{CaSO}_{4} \rightarrow \mathrm{CaO}+\mathrm{SO}_{3}(1400-1700 \mathrm{~K}) \\
& \mathrm{SO}_{3} \rightarrow \mathrm{SO}_{2}+1 / 2 \mathrm{O}_{2}
\end{aligned}
$$

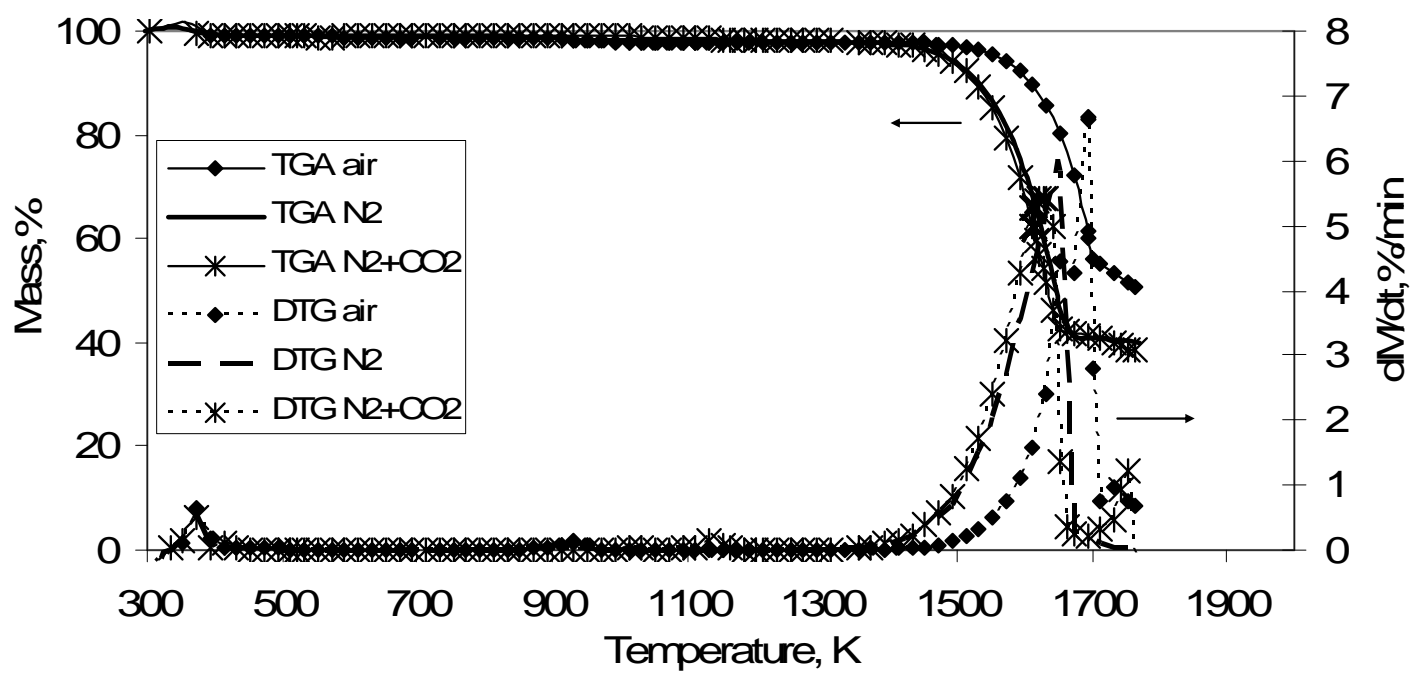

Figure 6.1 TGA and DTG profiles of $\mathrm{CaSO}_{4}$ decomposition in $\mathrm{N}_{2}, \mathrm{~N}_{2} / \mathrm{CO}_{2}$ mixture and air

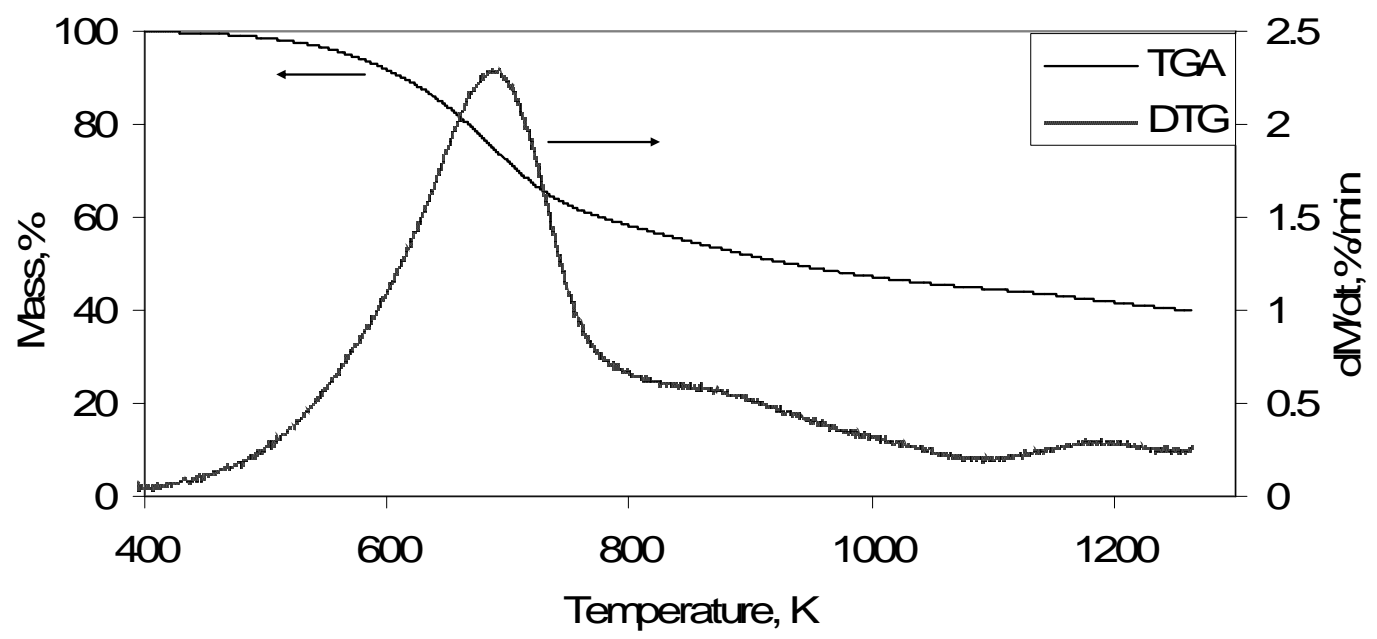

Figure 6.2 TGA and DTG profiles of thermal decomposition of $\mathrm{CaSO}_{4}+\mathrm{L} 1$ in a TGA in nitrogen atmosphere 


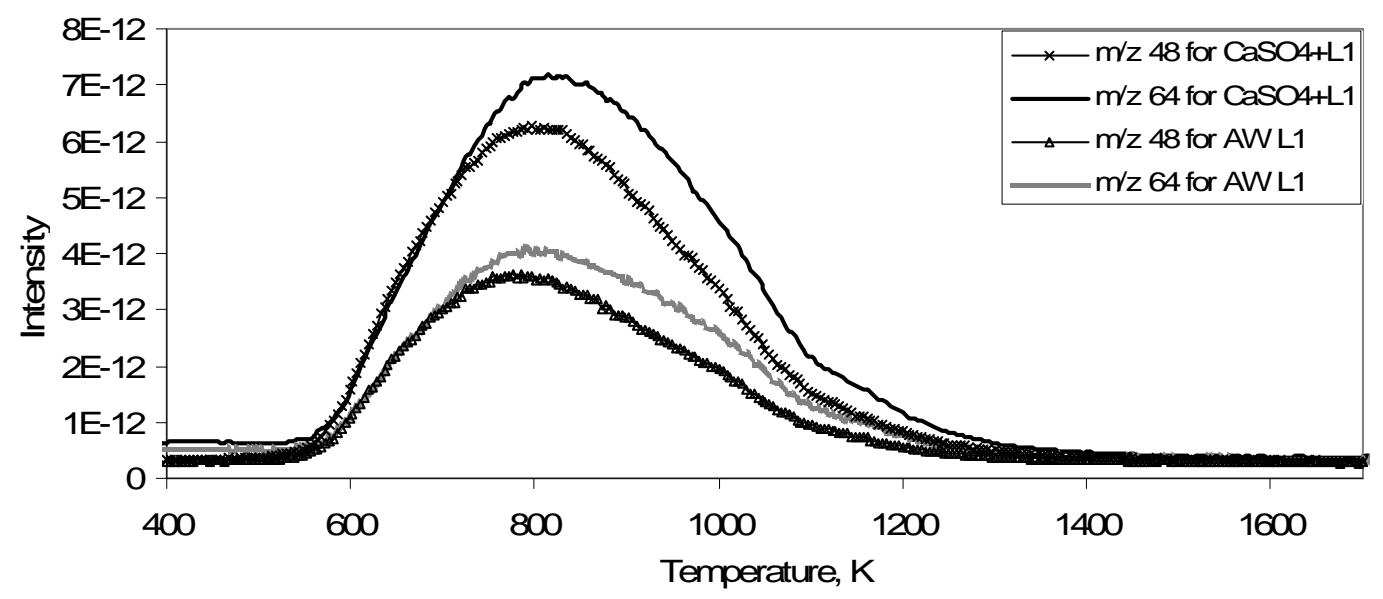

Figure $6.3 \mathrm{SO}_{2}$ evolution (m/z 64 and m/z 48) during pyrolysis of AW L1 and $\mathrm{CaSO}_{4}+\mathrm{L} 1$ in a TGA-MS

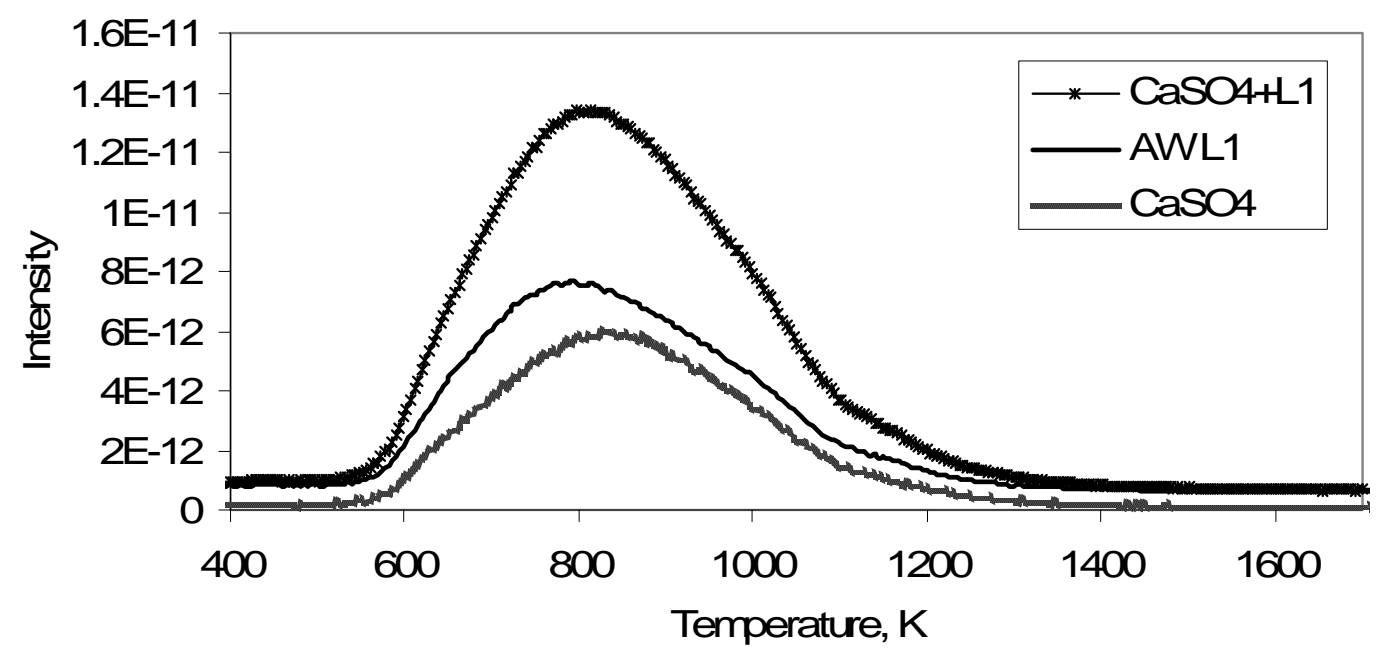

Figure 6.4 $\mathrm{SO}_{2}$ evolution during pyrolysis of $\mathrm{AW} \mathrm{L1}$ and $\mathrm{CaSO}_{4}+\mathrm{L} 1$ and from the decomposition of $\mathrm{CaSO}_{4}$

To study the transformation of calcium sulphate during lignite pyrolysis, pure $\mathrm{CaSO}_{4}$ was loaded into acid washed L1 which contains no pyrite and sulphate sulphur and only contain very low organic sulphur $(0.21 \% \mathrm{db})$, the sample is denoted as $\mathrm{CaSO}_{4}+\mathrm{L} 1$. Figure 6.2 shows TGA and differential thermogravimetric analysis (DTG) profiles of $\mathrm{CaSO}_{4}+\mathrm{L} 1$ decomposition in nitrogen at a heating rate of $10 \mathrm{~K} \cdot \mathrm{min}^{-1}$. It can be seen 
Chapter 6

from the TGA profile that $\mathrm{CaSO}_{4}+\mathrm{L} 1$ experiences rapid decomposition between 500 $800 \mathrm{~K}$, then above $800 \mathrm{~K}$ the mass of the char remains constant. From the DTG profile, it can be observed that there is one single peak with two shoulders around 800-900 K and 1100-1300 K. The single peak can be associated with lignite decomposition [58].

To determine at what temperature $\mathrm{CaSO}_{4}$ decomposes during lignite pyrolysis, $\mathrm{CaSO}_{4}+\mathrm{L} 1$ was pyrolysed in a TGA coupled with an MS (TGA-MS) under argon atmosphere at a heating rate of $10 \mathrm{~K} \cdot \mathrm{min}^{-1}$. To compare the effect of loaded calcium sulphate to the sample, AW L1 was also studied under the same condition in a TGAMS. Figure 6.3 shows that sulphur dioxide is evolved between 550 and $1250 \mathrm{~K}$ during pyrolysis of $\mathrm{CaSO}_{4}+\mathrm{L} 1$ and $\mathrm{AW} \mathrm{L1}$.

During pyrolysis, only $\mathrm{SO}_{2}$ was detected as the sulphurous gas evolved. The mass spectroscopic signals recorded for $\mathrm{SO}_{2}$ are the sum of $\mathrm{m} / \mathrm{z} 64\left(\mathrm{SO}_{2}{ }^{+}\right)$and $\mathrm{m} / \mathrm{z} 48\left(\mathrm{SO}^{+}\right)$, if both profiles show exactly the same shape [38].Although in small part, L1 contains organic sulphur which during pyrolysis most of the organic sulphur decomposes to evolve hydrogen sulphide $[4,8,31,43,101,118-120]$. However during the pyrolysis there was no $\mathrm{H}_{2} \mathrm{~S}$ gas detected (m/z 33 and m/z 34). L1 is a lignite with high oxygenated functional structures as already discussed in Chapter 4 and Chapter 5. The small amount of organic sulphur in AW L1 decomposes to form - $\mathrm{SH}$ radicals some of which may undergo complex reactions with organic matrix of the lignite which has high oxygenated structures leading to the formation of $\mathrm{SO}_{2}[9,109] . \mathrm{SO}_{2}$ yielded from calcium sulphate decomposition is not an intermediate product, it is formed from the decomposition reaction of calcium sulphate as described in R6.3 and R6.4, thus there is no reaction between char and $\mathrm{SO}_{2}$ to produce new organic sulphur in the char [9]. 
It can be observed from Figure 6.3 that the evolution of $\mathrm{SO}_{2}$ in both $\mathrm{AW} \mathrm{L} 1$ and $\mathrm{CaSO}_{4}+\mathrm{L} 1$ occurs in the same temperature range, that is between 550 and $1250 \mathrm{~K}$. As mentioned previously, pure $\mathrm{CaSO}_{4}$ decomposition occurs between 1400 and $1700 \mathrm{~K}$. In the presence of lignite organic matrix, $\mathrm{CaSO}_{4}$ decomposes at much lower temperature $[9,64]$. Previous studies reported that $\mathrm{CaSO}_{4}$ in weathered coal starts to decompose at $623 \mathrm{~K}[60,110]$. By subtracting the sulphur dioxide, which is the sum of $\mathrm{m} / \mathrm{z} 64$ and 48, evolved during pyrolysis of $\mathrm{CaSO}_{4}+\mathrm{L} 1$ with the sum of $\mathrm{m} / \mathrm{z} 64$ and $\mathrm{m} / \mathrm{z} 48$ evolved during pyrolysis of AW L1, the evolution of sulphur dioxide from the decomposition of $\mathrm{CaSO}_{4}$ can be observed. Figure 6.4 shows the evolution of $\mathrm{SO}_{2}$ from $\mathrm{CaSO}_{4}+\mathrm{L} 1, \mathrm{AW}$ $\mathrm{L} 1$ and the evolution of $\mathrm{SO}_{2}$ from the decomposition of $\mathrm{CaSO}_{4}$. It can be observed that during pyrolysis of lignite loaded with calcium sulphate, $\mathrm{CaSO}_{4}$ also starts to decompose at ca $623 \mathrm{~K}$. 


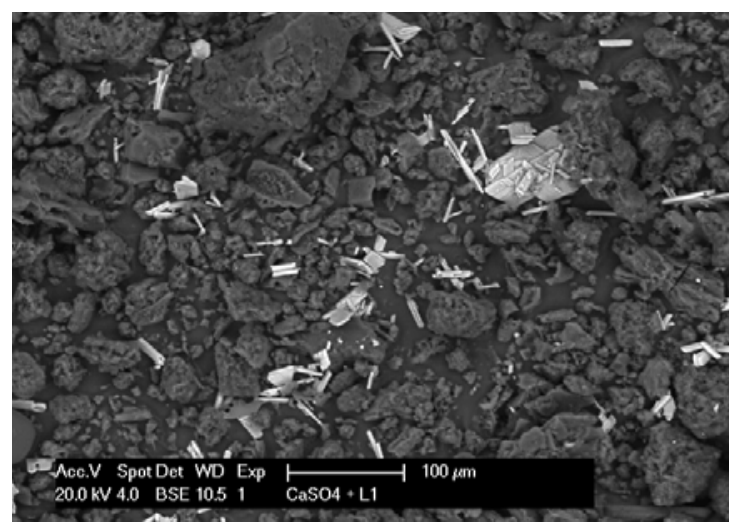

(a)

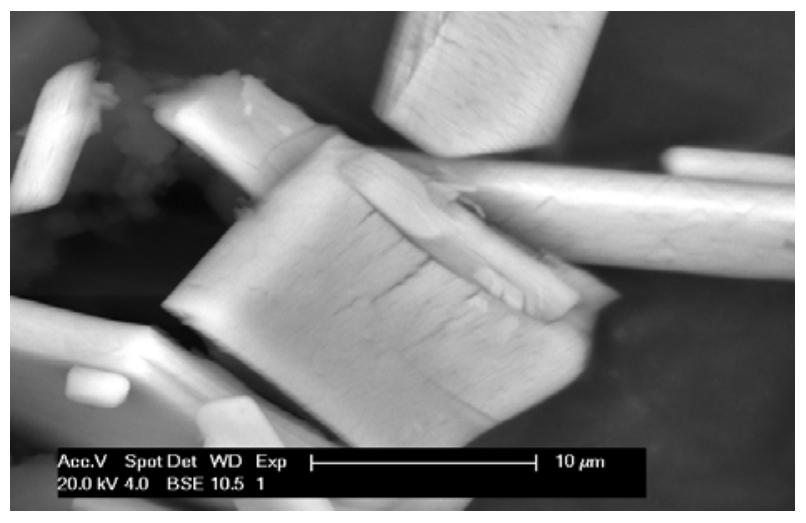

(b)

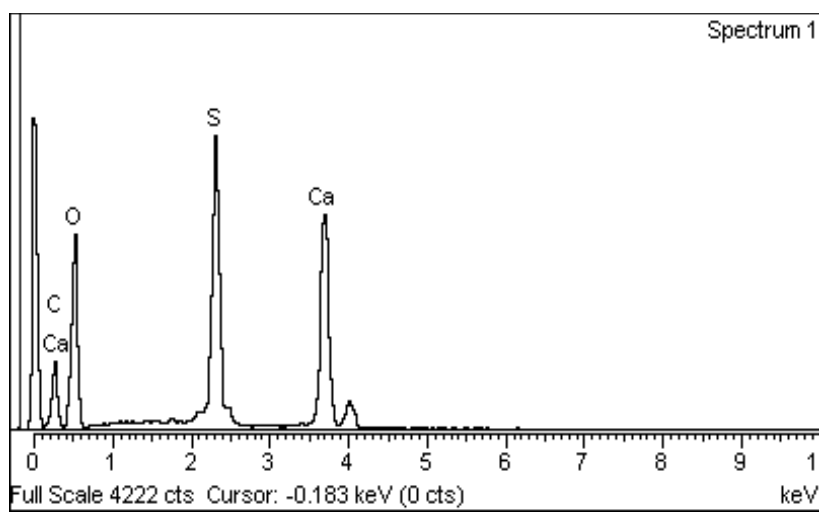

( c )

Figure 6.5 SEM backscattered images of $\mathrm{CaSO}_{4}+\mathrm{L} 1$ low magnification and high magnification (a-b) and its EDS spectra ( c ) 


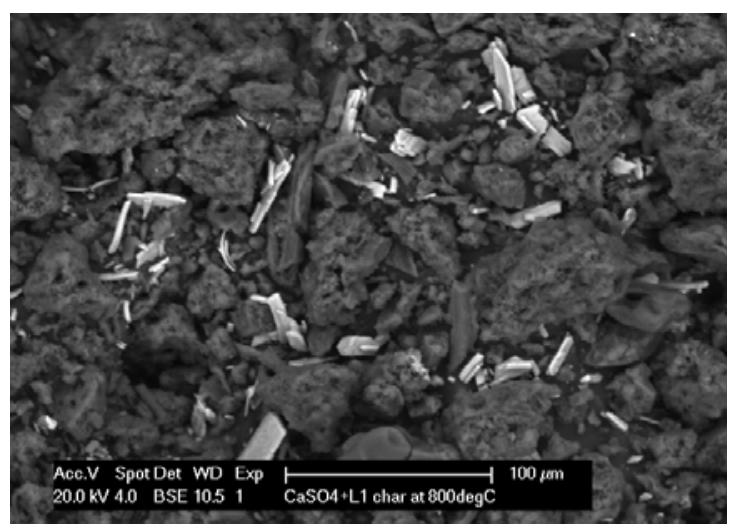

(a)

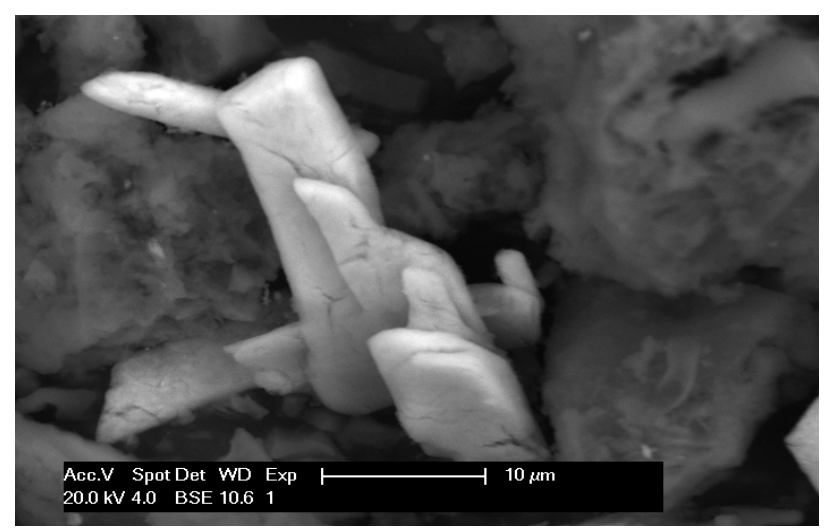

(b)

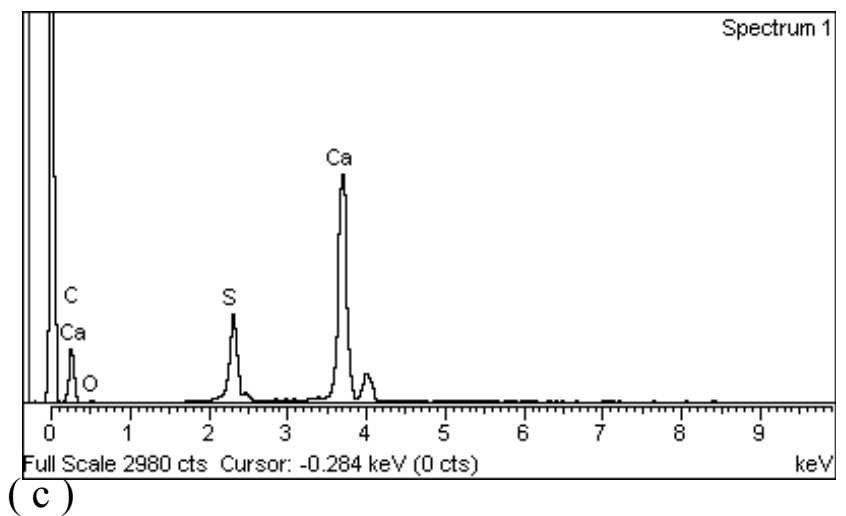

Figure 6.6 SEM backscattered images $\mathrm{CaSO}_{4}+\mathrm{L} 1$ chars produced at $1073 \mathrm{~K}$ low and high magnification (a-b) and its EDS spectra ( c ) 


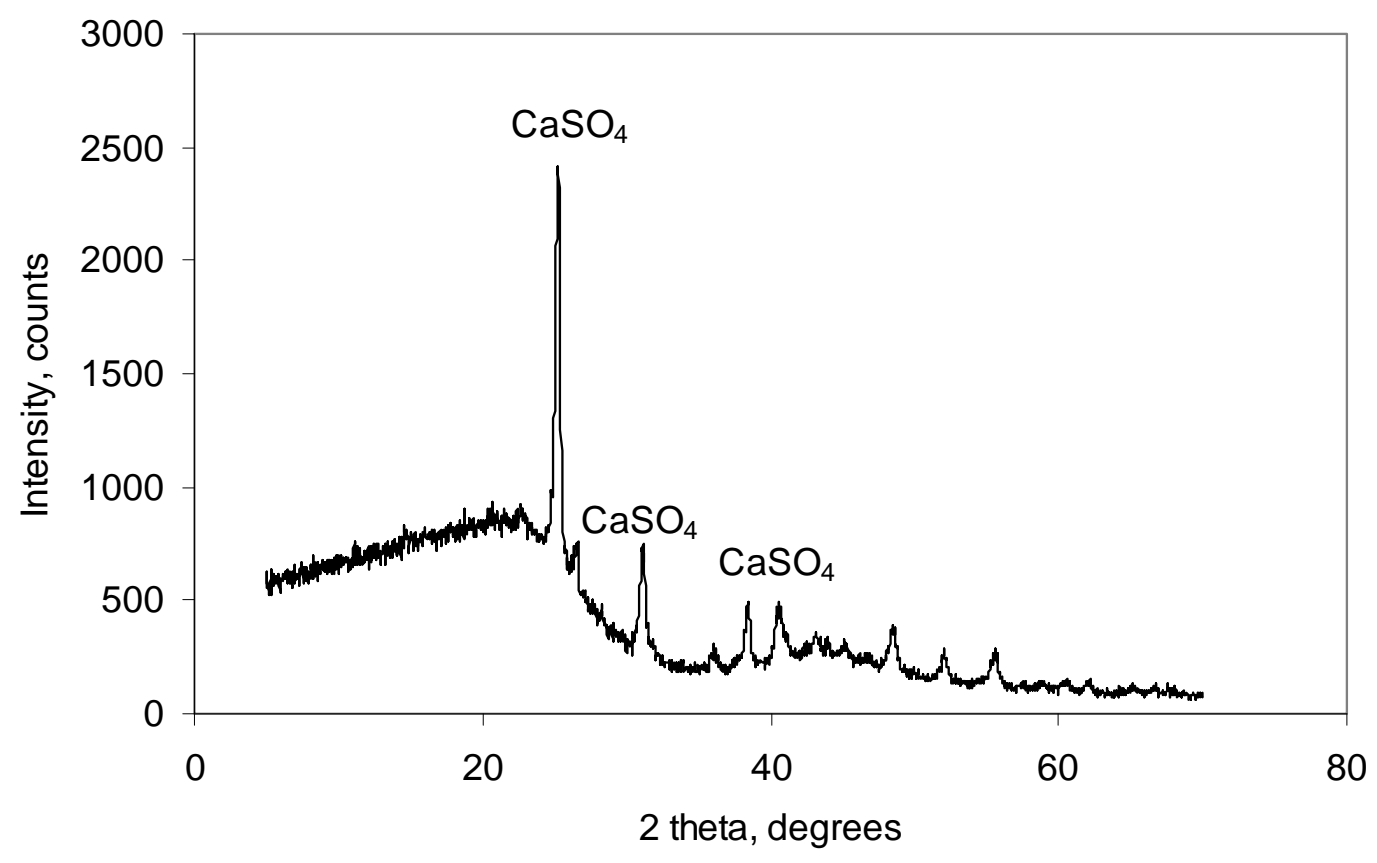

Figure 6.7 XRD patterns of the char of $\mathrm{CaSO}_{4}+\mathrm{L} 1$ produced at $1073 \mathrm{~K}$

The intensity of the evolution of $\mathrm{SO}_{2}$ from the decomposition of $\mathrm{CaSO}_{4}$ shown in Figure 6.4 is quite low. To confirm that only a small part of $\mathrm{CaSO}_{4}$ is decomposed, the CaSO4+L1 sample and its chars was analysed using SEM, SEM-EDS as well as XRD. SEM images of $\mathrm{CaSO}_{4}+\mathrm{L} 1$ and its EDS spectra are shown in Figure 6.5, while SEM images of $\mathrm{CaSO}_{4}+\mathrm{L} 1$ char produced at $1073 \mathrm{~K}$ and its EDS spectra are presented in Figure 6.6. $\mathrm{CaSO}_{4}$ can be observed as indicated by the white spots in the SEM images and also confirmed by the EDS spectra in Figure 6.5. From Figure 6.6 it can be seen that $\mathrm{CaSO}_{4}$ is still observable in the char produced at $1073 \mathrm{~K}$. The presence of $\mathrm{CaSO}_{4}$ in the char of $\mathrm{CaSO}_{4}+\mathrm{L} 1$ produced at $1073 \mathrm{~K}$ is also confirmed from XRD spectra shown in Figure 6.7. The low intensity of $\mathrm{SO}_{2}$ observed from the decomposition of $\mathrm{CaSO}_{4}$ is due to only small part of $\mathrm{CaSO}_{4}$ decomposed during pyrolysis of $\mathrm{CaSO}_{4}+\mathrm{L} 1 . \mathrm{CaSO}_{4}$ is a very stable compound, it is the least volatile sulphate among sulphates commonly found in coal [112]. 
Chapter 6

A calcium based sorbent such as limestone $\left(\mathrm{CaCO}_{3}\right)$ has been widely used to directly capture $\mathrm{SO}_{2}$ during combustion or gasification $[64,121]$. The sulphation reaction can proceed via indirect or indirect reactions as follows [121]:

Indirect sulphation $\quad: \mathrm{CaCO}_{3} \rightarrow \mathrm{CaO}_{(\mathrm{s})}+\mathrm{CO}_{2(\mathrm{~g})}$

$$
\mathrm{CaO}_{(\mathrm{s})}+\mathrm{SO}_{2(\mathrm{~g})}+1 / 2 \mathrm{O}_{2(\mathrm{~g})} \rightarrow \mathrm{CaSO}_{4(\mathrm{~s})}
$$

Direct sulphation $\quad: \mathrm{CaCO}_{3(\mathrm{~s})}+\mathrm{SO}_{2(\mathrm{~g})}+1 / 2 \mathrm{O}_{2(\mathrm{~g})} \rightarrow \mathrm{CaSO}_{4(\mathrm{~s})}+\mathrm{CO}_{2(\mathrm{~g})}$

In both sulphation reactions (R6.5-R6.7), $\mathrm{CaSO}_{4}$ is formed as the final product, this imply that $\mathrm{CaSO}_{4}$ is a very stable compound which is very hard to decompose at high temperature.

\subsubsection{Iron sulphates decomposition}

The decomposition of pure $\mathrm{FeSO}_{4} \cdot 7 \mathrm{H}_{2} \mathrm{O}$ and $\mathrm{Fe}_{2}\left(\mathrm{SO}_{4}\right)_{3} \cdot 9 \mathrm{H}_{2} \mathrm{O}$ were also studied in the three different atmospheres in TGA. Figure 6.8 shows the decomposition of $\mathrm{Fe}_{2}\left(\mathrm{SO}_{4}\right)_{3}$. It can be seen that the decomposition of ferric sulphate in $\mathrm{N} 2, \mathrm{~N} 2 / \mathrm{CO} 2$ mixture, and air shows the similar behaviour. The mass loss below $473 \mathrm{~K}$ seems from the release of water, the decomposition of ferric sulphate itself occurs at temperatures between 830 $980 \mathrm{~K}$. The decomposition of $\mathrm{Fe}_{2}\left(\mathrm{SO}_{4}\right)_{3} .9 \mathrm{H}_{2} \mathrm{O}$ can be described as follows [38]:

$$
\begin{aligned}
& \mathrm{Fe}_{2}\left(\mathrm{SO}_{4}\right)_{3} \cdot 9 \mathrm{H}_{2} \mathrm{O} \rightarrow \mathrm{Fe}_{2}\left(\mathrm{SO}_{4}\right)_{3}+9 \mathrm{H}_{2} \mathrm{O}(<473 \mathrm{~K}) \\
& \mathrm{Fe}_{2}\left(\mathrm{SO}_{4}\right)_{3} \rightarrow \mathrm{Fe}_{2} \mathrm{O}_{3}+3 \mathrm{SO}_{3} \\
& \mathrm{SO}_{3} \rightarrow \mathrm{SO}_{2}+1 / 2 \mathrm{O}_{2}
\end{aligned}
$$




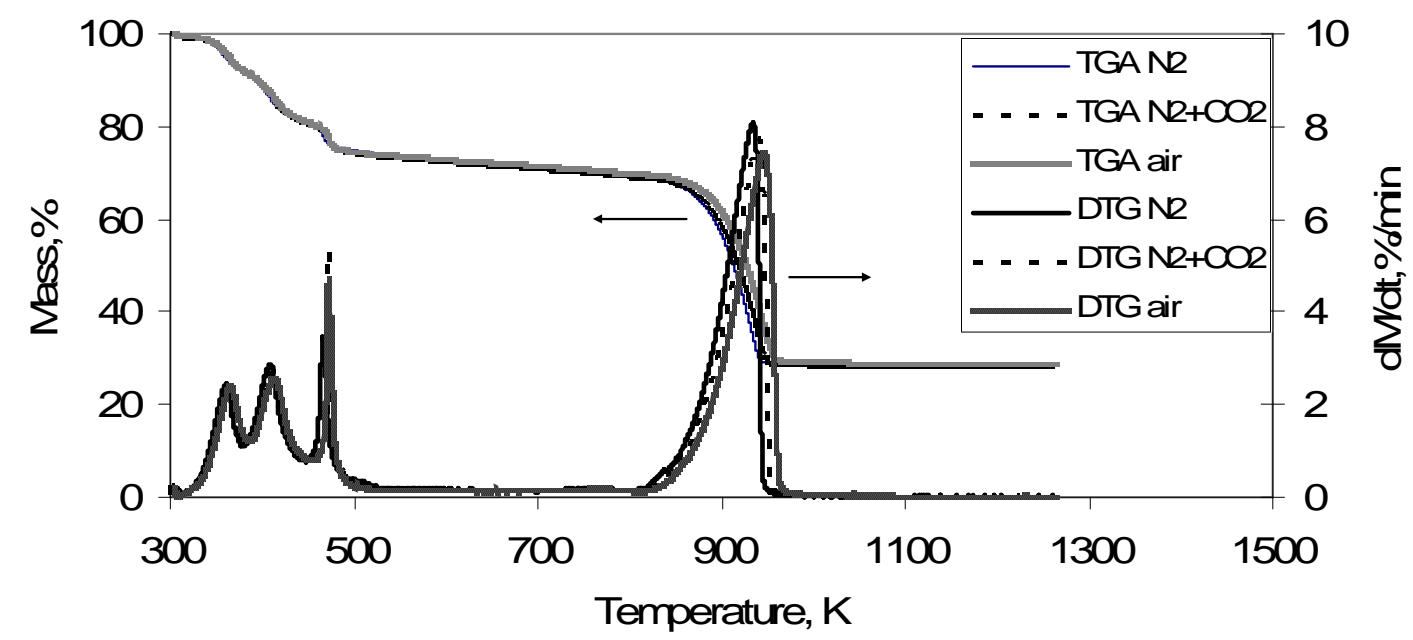

Figure 6.8 TGA and DTG profiles of $\mathrm{Fe}_{2}\left(\mathrm{SO}_{4}\right)_{3}$ decomposition in $\mathrm{N}_{2}, \mathrm{~N}_{2} / \mathrm{CO}_{2}$ mixture and air

The decomposition of pure $\mathrm{FeSO}_{4} \cdot 7 \mathrm{H}_{2} \mathrm{O}$ was studied under the same three different atmospheres in a TGA with a constant heating rate of 10 K. $\mathrm{min}^{-1}$ from room temperature to $1273 \mathrm{~K}$. The results are presented in Figure 6.9. The thermal decomposition of ferrous sulphate is very similar in the three different atmospheres. It can be seen that there is a significant mass loss between room temperature and $413 \mathrm{~K}$ and between 523 and $543 \mathrm{~K}$. The mass loss in these temperature ranges relates to the water evaporation from $\mathrm{FeSO}_{4} \cdot 7 \mathrm{H}_{2} \mathrm{O}$. Another significant mass loss occurs between 813 and $953 \mathrm{~K}$. This change is attributed to the decomposition of $\mathrm{FeSO}_{4}$ to form iron oxide and release sulphur dioxide [38]. The decomposition of ferrous sulphate thus can be described as:

$\mathrm{FeSO}_{4} .7 \mathrm{H}_{2} \mathrm{O} \rightarrow \mathrm{FeSO}_{4}+7 \mathrm{H}_{2} \mathrm{O}(<543 \mathrm{~K})$

$\mathrm{FeSO}_{4} \rightarrow \mathrm{Fe}_{2} \mathrm{O}_{3}+\mathrm{SO}_{3}+\mathrm{SO}_{2} \quad(813-953 \mathrm{~K})$

$\mathrm{SO}_{3} \rightarrow \mathrm{SO}_{2}+1 / 2 \mathrm{O}_{2}$ 


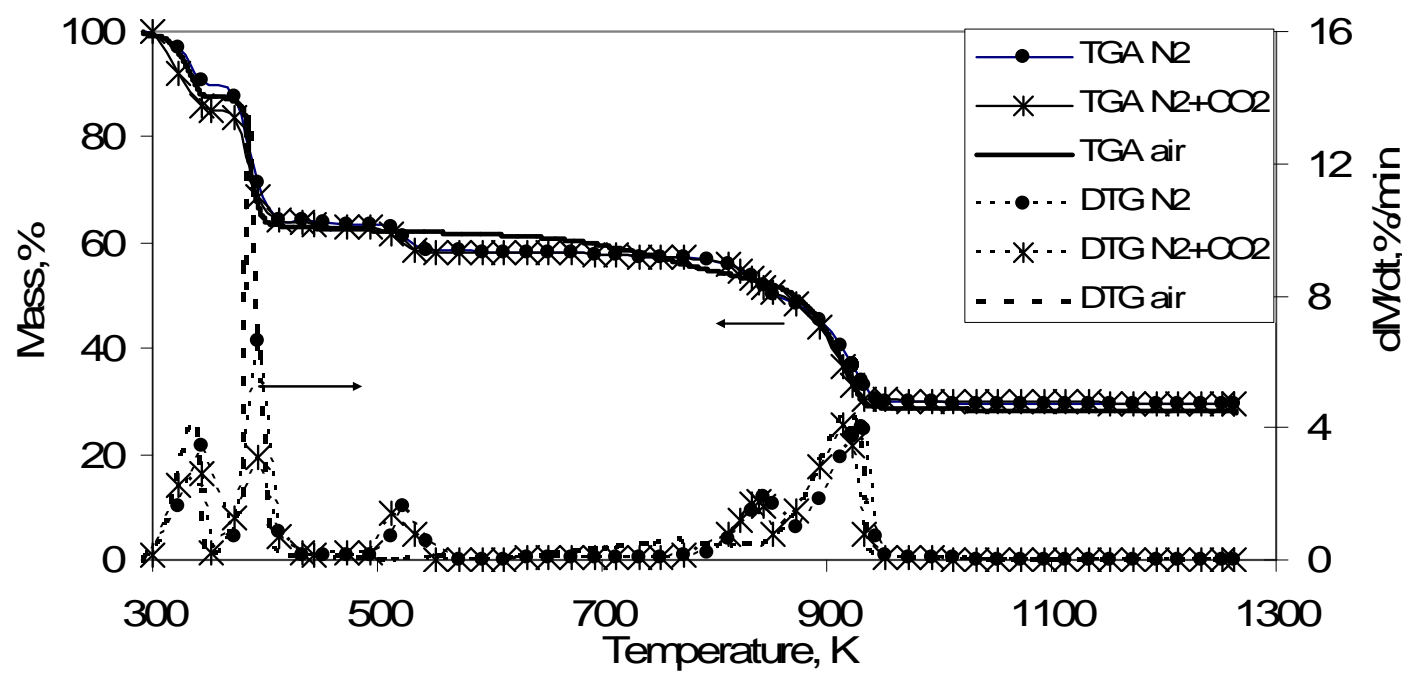

Figure 6.9 TGA and DTG profiles of $\mathrm{FeSO}_{4}$ decomposition in $\mathrm{N}_{2}, \mathrm{~N}_{2} / \mathrm{CO}_{2}$ mixture and air

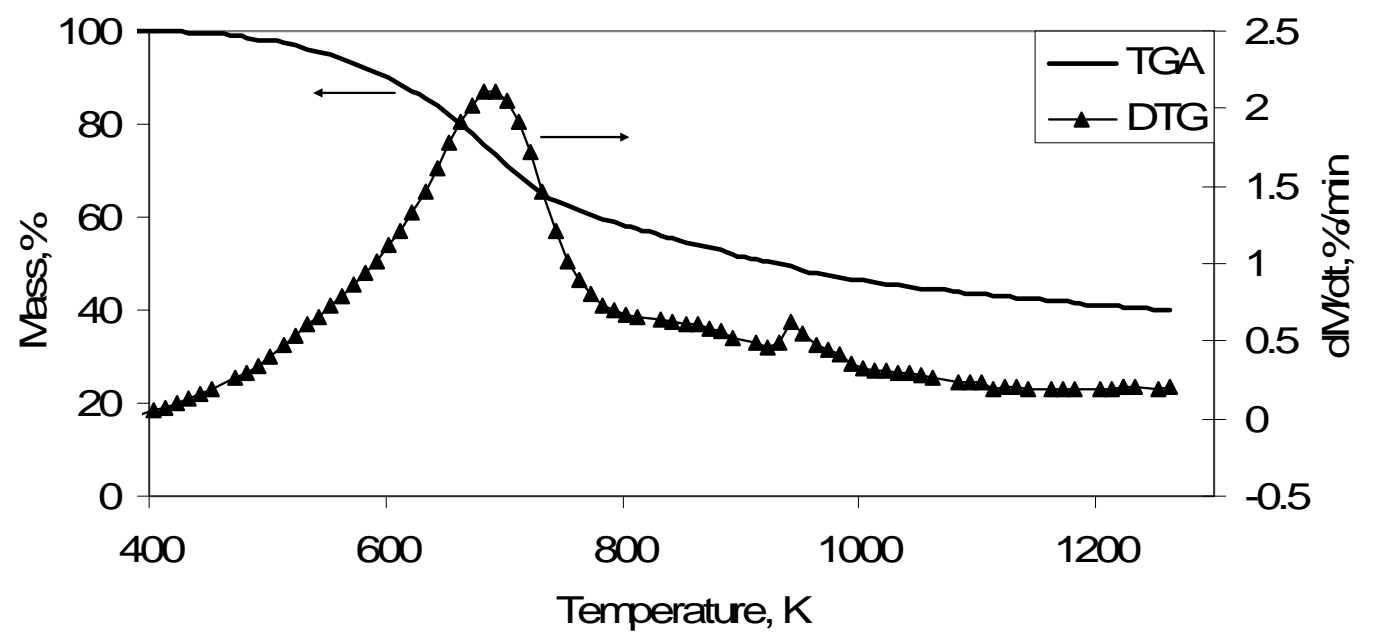

Figure 6.10 TGA and DTG profiles of the pyrolysis of $\mathrm{FeSO}_{4}+\mathrm{L} 1$ in nitrogen atmosphere. 


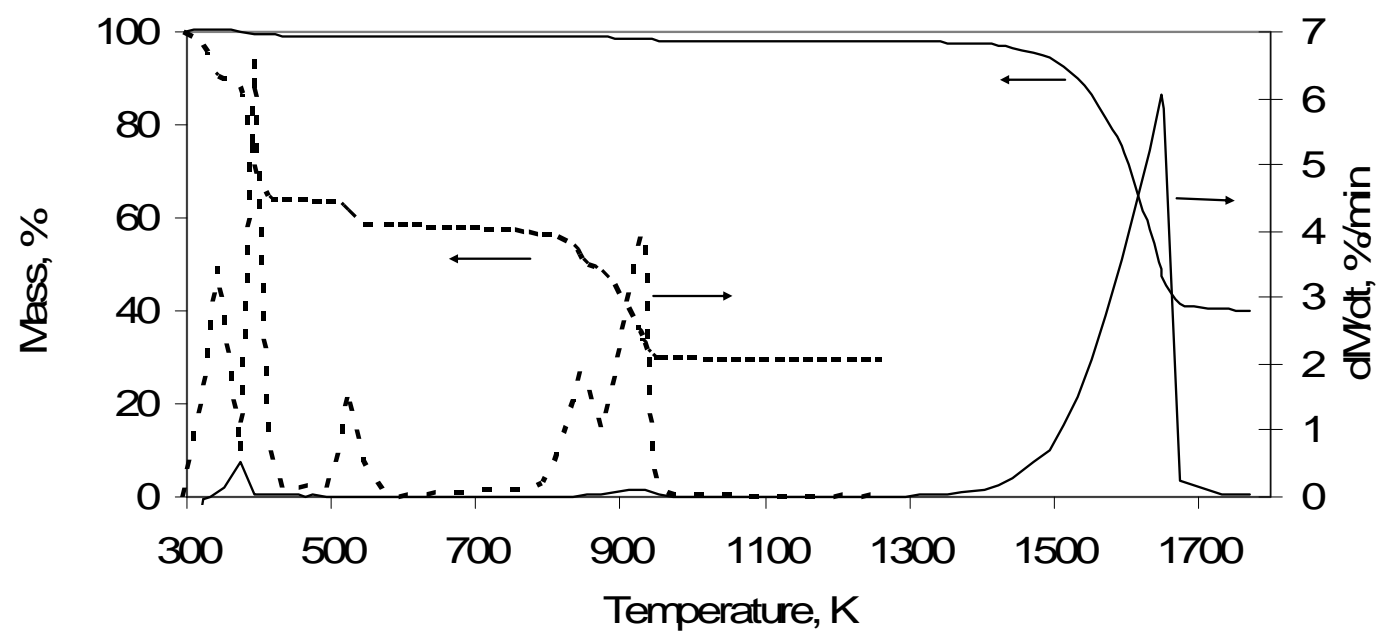

Figure 6.11 Comparison of the thermal decomposition of pure $\mathrm{CaSO}_{4}$ (solid line (-)) and FeSO4 (broken line (---)) in a TGA under nitrogen

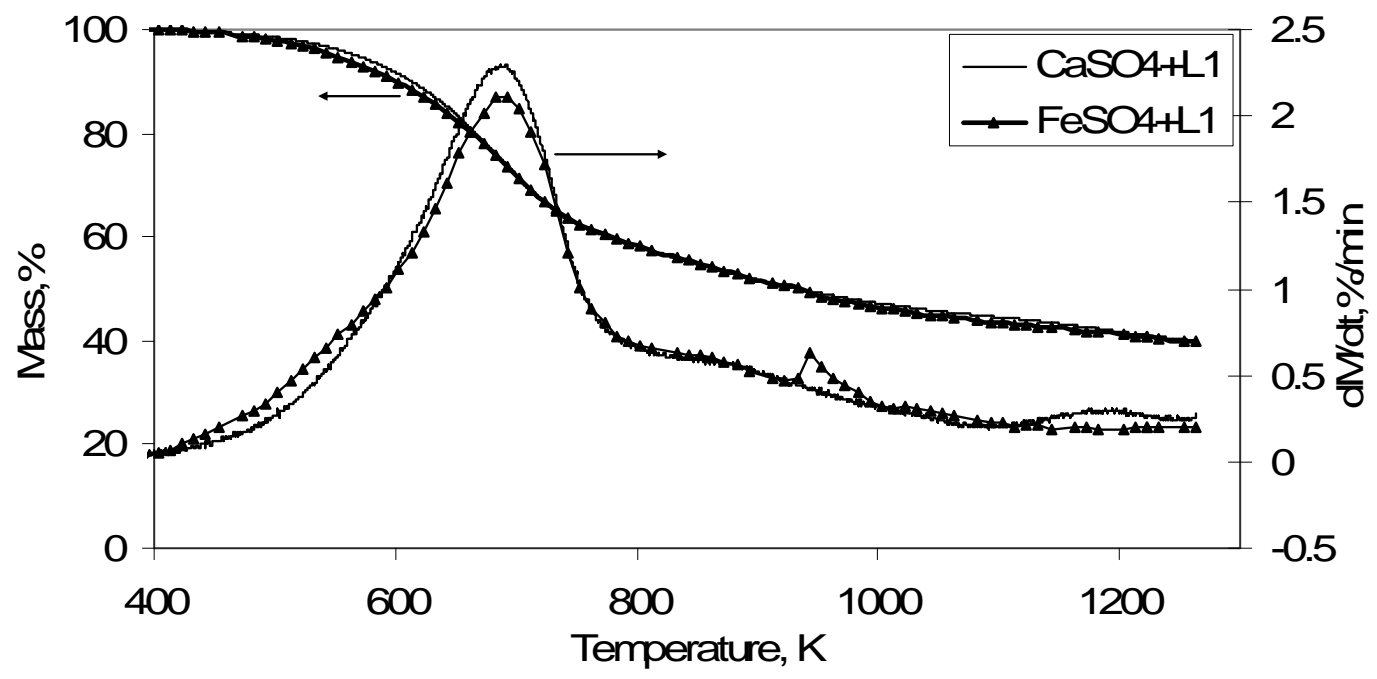

Figure 6.12 Comparison of the thermal decomposition of $\mathrm{CaSO}_{4}+\mathrm{L} 1$ and $\mathrm{FeSO}_{4}+\mathrm{L} 1$ in a TGA under nitrogen atmosphere. 


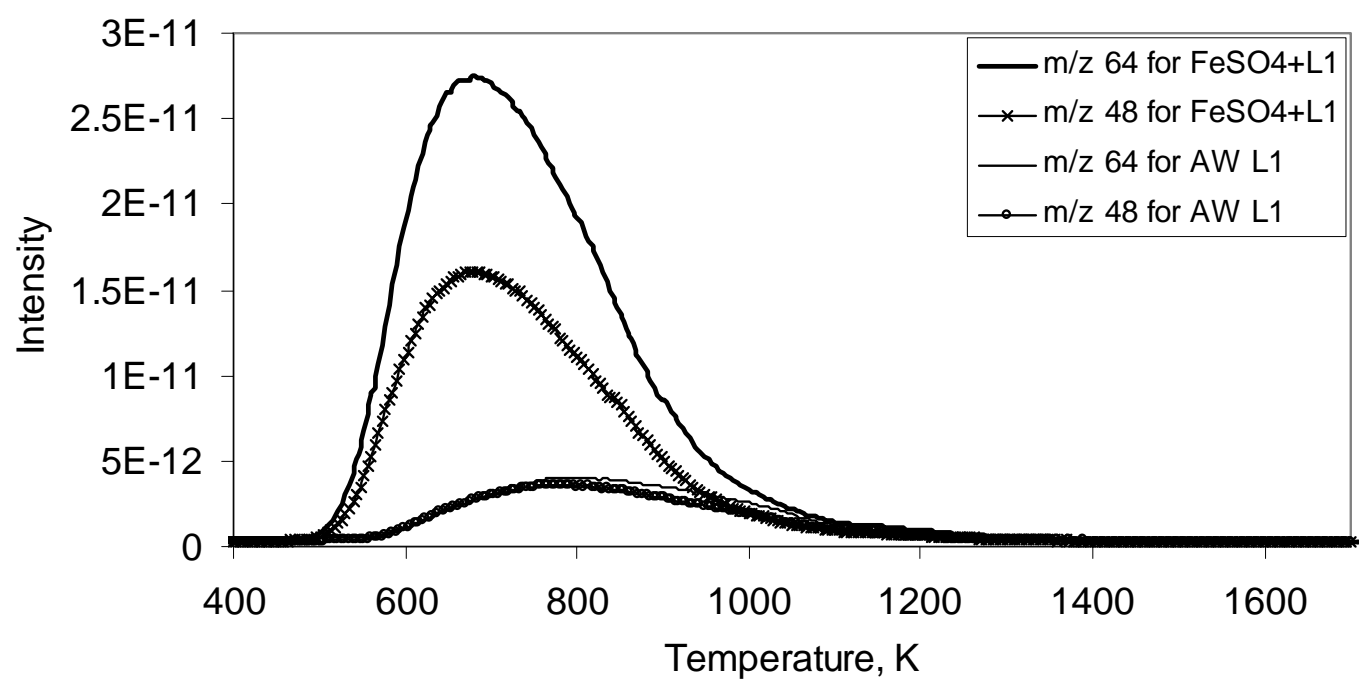

Figure $6.13 \mathrm{SO}_{2}$ evolution during pyrolysis of $\mathrm{FeSO}_{4}+\mathrm{L} 1$ and $\mathrm{AW} \mathrm{L} 1$

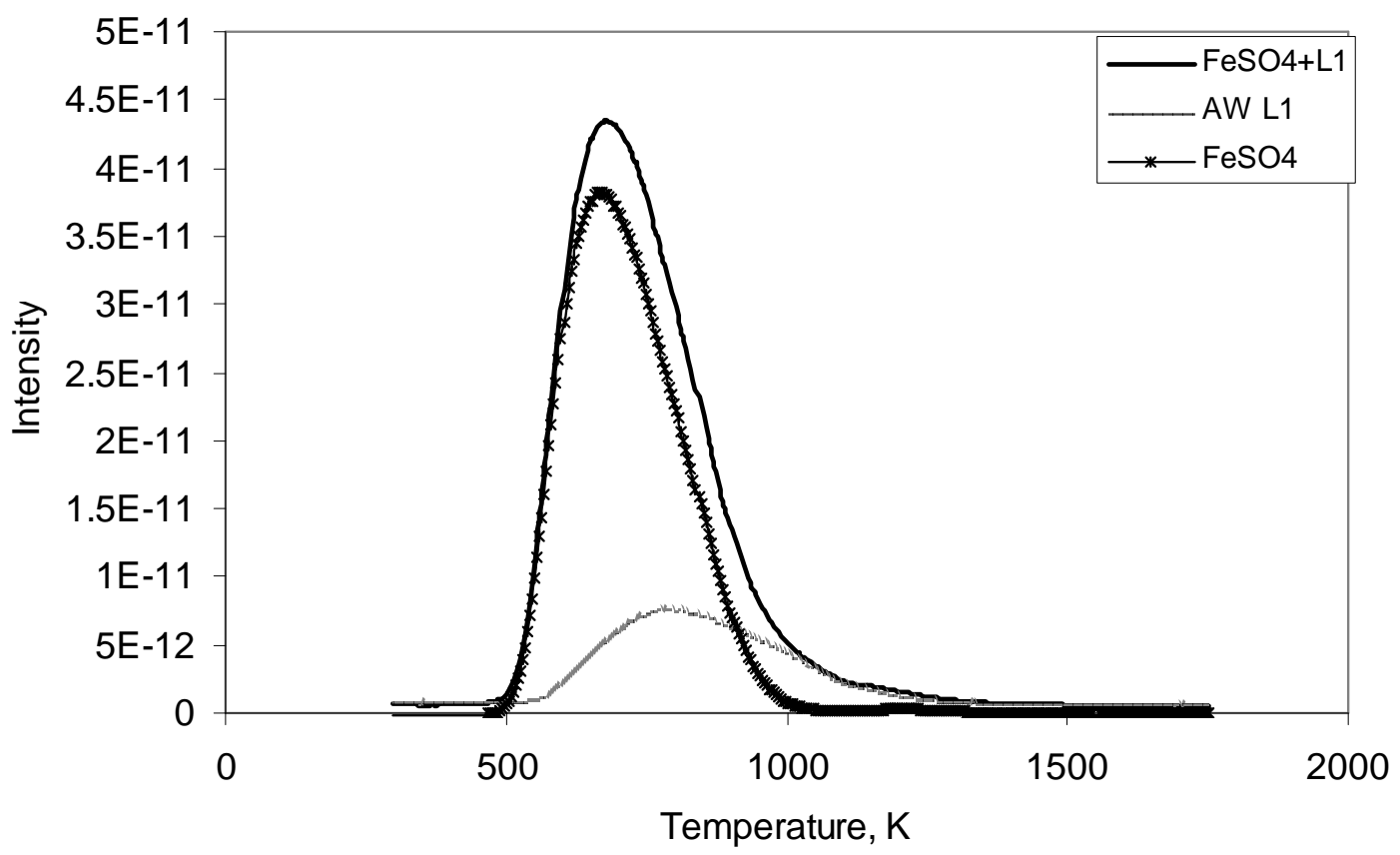

Figure $6.14 \mathrm{SO}_{2}$ evolution, sum of $\mathrm{m} / \mathrm{z} 64+\mathrm{m} / \mathrm{z} 48$, during pyrolysis of $\mathrm{AW}$ $\mathrm{L} 1$ and $\mathrm{FeSO}_{4}+\mathrm{L} 1$ and from the decomposition of $\mathrm{FeSO}_{4}$ 
Chapter 6

Ferrous sulphate was also loaded to AW L1 and the sample is named as $\mathrm{FeSO}_{4}+\mathrm{L} 1$. Figure 6.10 shows TGA and DTG thermograms of the pyrolysis of $\mathrm{FeSO}_{4}+\mathrm{L} 1$ in a TGA under nitrogen at a flow rate of $100 \mathrm{ml} \cdot \mathrm{min}^{-1}$ at a constant heating rate of $10 \mathrm{~K} \cdot \mathrm{min}^{-1}$ from room temperature to $1273 \mathrm{~K}$. From the TGA profile, it can be seen that a significant mass loss occurs between $500-800 \mathrm{~K}$. A major DTG peak is also observed between $500-800 \mathrm{~K}$, this peak can be related to the lignite decomposition [58].

Comparing the decomposition of pure calcium sulphate and ferrous sulphate as shown in Figure 6.11, it can be seen that they have a very different decomposition temperature range. As discussed previously, pure sulphate decomposes in the temperature range of 1400-1700 K, while pure $\mathrm{FeSO}_{4}$ decomposes in the temperature range of 813-953 K. However in the lignite samples loaded with pure sulphate the thermal decompositions are quite similar as shown in Figure 6.12. When surrounding with organic matrix, sulphate decomposes at lower temperature $[9,64]$.

To determine the decomposition temperature of ferrous sulphate during lignite pyrolysis, $\mathrm{FeSO}_{4}+\mathrm{L} 1$ was pyrolysed in a TGA-MS in argon at a flow rate of 100 $\mathrm{ml} \cdot \mathrm{min}^{-1}$ at a constant heating rate of $10 \mathrm{~K} \cdot \mathrm{min}^{-1}$. During the pyrolysis, again, $\mathrm{H}_{2} \mathrm{~S}$ with $\mathrm{m} / \mathrm{z} 33$ and $\mathrm{m} / \mathrm{z} 34$ is not detected. The only sulphur gas observed during the pyrolysis was sulphur dioxide $(\mathrm{m} / \mathrm{z} 64$ and $\mathrm{m} / \mathrm{z} 48)$. The profiles of $\mathrm{SO}_{2}$ evolution during pyrolysis of $\mathrm{FeSO}_{4}+\mathrm{L} 1$ and AW L1 are shown in Figure 6.13. It can be observed that $\mathrm{SO}_{2}$ starts to evolve at approximately $500 \mathrm{~K}$.

The profile of $\mathrm{SO}_{2}$ from the decomposition of $\mathrm{FeSO}_{4}$ in $\mathrm{FeSO}_{4}+\mathrm{L} 1$ is calculated by subtracting $\mathrm{SO}_{2}$ evolved during pyrolysis of $\mathrm{FeSO}_{4}+\mathrm{L} 1$ with $\mathrm{SO}_{2}$ evolved during pyrolysis of AW L1 as shown in Figure 6.14. Due to the higher volatility of $\mathrm{FeSO}_{4}$, it decomposes at lower temperature and releases a larger quantity of $\mathrm{SO}_{2}$ than $\mathrm{CaSO}_{4}[64$, 
112]. The evolution of $\mathrm{SO}_{2}$ increases sharply from ca $500 \mathrm{~K}$, peaks at $680 \mathrm{~K}$ and then decreases significantly until $1000 \mathrm{~K}$ when there is no significant $\mathrm{SO}_{2}$ evolution anymore.

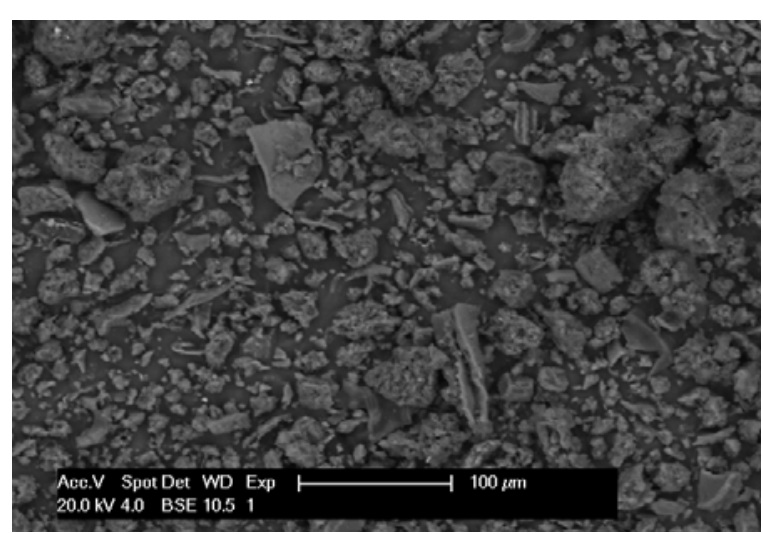

(a)

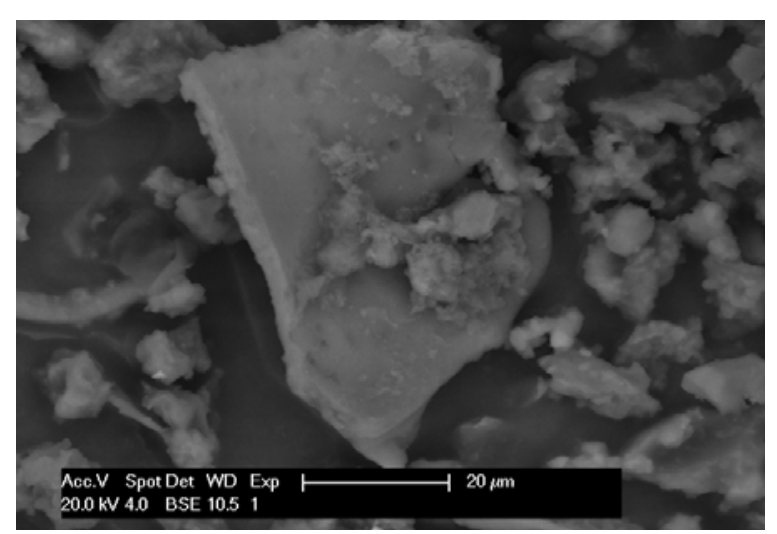

(b)

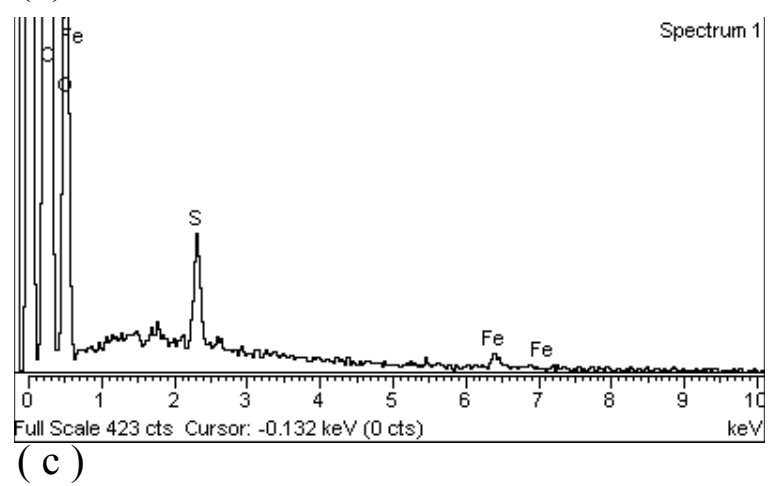

Figure 6.15 SEM backscattered images of $\mathrm{FeSO}_{4}+\mathrm{L} 1$ low and high magnification (a-b) and its EDS spectra ( c )

Figure 6.15 presents SEM images and its EDS spectra of $\mathrm{FeSO}_{4}+\mathrm{L} 1$ while the chars produced at $1073 \mathrm{~K}$ and its EDS spectra are presented in Figure 6.16. From the EDS spectra it can be observed that the intensity of $\mathrm{S}$ atom reduces significantly in the char compared to the $\mathrm{S}$ intensity in raw $\mathrm{FeSO}_{4}+\mathrm{L} 1$. The presence of $\mathrm{Fe}$ and $\mathrm{S}$ in this char indicates that troilite $(\mathrm{FeS})$ could have been formed from the decomposition of $\mathrm{FeSO}_{4}$ [38]. The formation of FeS can be described as follows:

$\mathrm{FeSO}_{4}+4 \mathrm{H}_{2} \rightarrow \mathrm{FeS}+4 \mathrm{H}_{2} \mathrm{O}$ 
However the presence of FeS in the remaining char cannot be detected by SEM-EDS or XRD directly. Another possibility is that FeSO4 may convert to complex sulphide which is not detectable by chemical analysis [110].

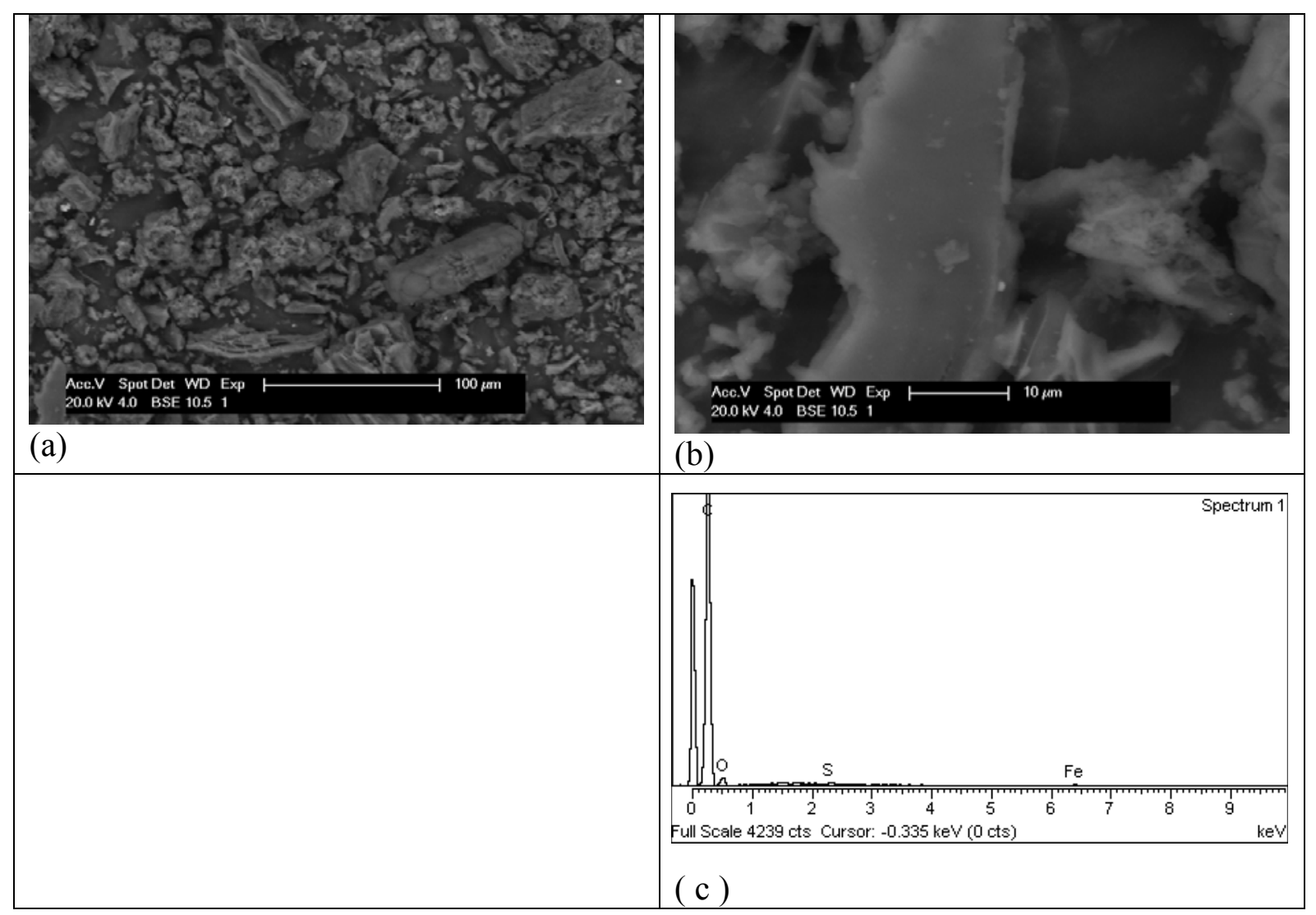

Figure 6.16 SEM backscattered images of $\mathrm{FeSO}_{4}+\mathrm{L} 1$ char produced at 1073K low and high magnification (a-b) and its EDS spectra ( c )

\subsubsection{Transformation of inherent sulphate during lignite pyrolysis}

The sulphur form in L4 is dominated by sulphate sulphur $(0.96 \%)$ and followed by pyritic sulphur $(0.40 \%)$. The organic sulphur presents in L4 is very low, it is only $0.02 \%$ (see Table 4.3 Chapter 4 ). Hence it is worth to study the transformation of inherent sulphate during lignite pyrolysis by using lignite with extremely high percentage of inherent sulphate and very low percentage of organic sulphur. 
Chapter 6

Figure 6.17 presents FTIR spectra of L4. Bands observed in the range of $3600-4000 \mathrm{~cm}^{-}$

${ }^{1}$ and $400-600 \mathrm{~cm}^{-1}$ are assigned to the occurrence of mineral and inorganic matter in the sample [88, 89]. As discussed in Chapter 4, the lignites, including L4, have a great abundance of oxygenated functional groups which are observed in the $1000-1800 \mathrm{~cm}^{-1}$ region $[17,18,87,88]$. The present of inorganic sulphate in the sample can also be detected by FTIR $[122,123]$. Bands observed in the range of $610-680 \mathrm{~cm}^{-1}$ and 1130 $1080 \mathrm{~cm}^{-1}$ could be assigned to ion sulphate. It can be seen from Figure 6.17 that there are broad bands in those regions confirming that there is a significance occurrence of sulphate in L4.

Gases evolved were recorded during pyrolysis of L4 in argon at a flow rate of 100 $\mathrm{ml} \cdot \mathrm{min}^{-1}$ and heating rate of $10 \mathrm{~K} \cdot \mathrm{min}^{-1}$ from room temperature to $1073 \mathrm{~K}$ in a TGAMS. The gaseous sulphur detected during pyrolysis was only sulphur dioxide which has $\mathrm{m} / \mathrm{z} 64$ and m/z 48, the $\mathrm{SO}_{2}$ evolved during pyrolysis of L4 is presented in Figure 6.18. It shows that $\mathrm{SO}_{2}$ starts to evolve at $473 \mathrm{~K}$, peaks at 738 and there is a shoulder around $917 \mathrm{~K}$, which after this temperature the evolution of $\mathrm{SO}_{2}$ decreases significantly since there is not much sulphur left to be decomposed.

The starting temperature of $\mathrm{SO}_{2}$ evolution in $\mathrm{L} 4$ is similar to the starting temperature of $\mathrm{SO}_{2}$ evolution from $\mathrm{FeSO}_{4}$ decomposition (see Figure 6.13 and 6.14 ). The presence of iron sulphates, both $\mathrm{FeSO}_{4}$ and $\mathrm{Fe}_{2}\left(\mathrm{SO}_{4}\right)_{3}$, in $\mathrm{L} 4$ can be confirmed from the mineral and inorganic forms data presented in Table 4.8 Chapter 4. It can be seen that the Fe content in $\mathrm{L} 4$ is more than the $\mathrm{Ca}$ content.

$\mathrm{FeSO}_{4}$ decomposition follows reaction R6.12-R6.13 as discussed in the previous section. $\mathrm{FeSO}_{4}$ may also be decomposed to form $\mathrm{FeS}$ and $\mathrm{H}_{2} \mathrm{O}$ as described in R6.14. In the form of $\mathrm{Fe}_{2} \mathrm{SO}_{4}$, the sulphate surrounded by carbon decomposes at the same thermal 
decomposition temperature of $\mathrm{FeSO}_{4}$ [112]. The decomposition of $\mathrm{Fe}_{2}\left(\mathrm{SO}_{4}\right)_{3}$ follows reactions R6.9 and R6.10.

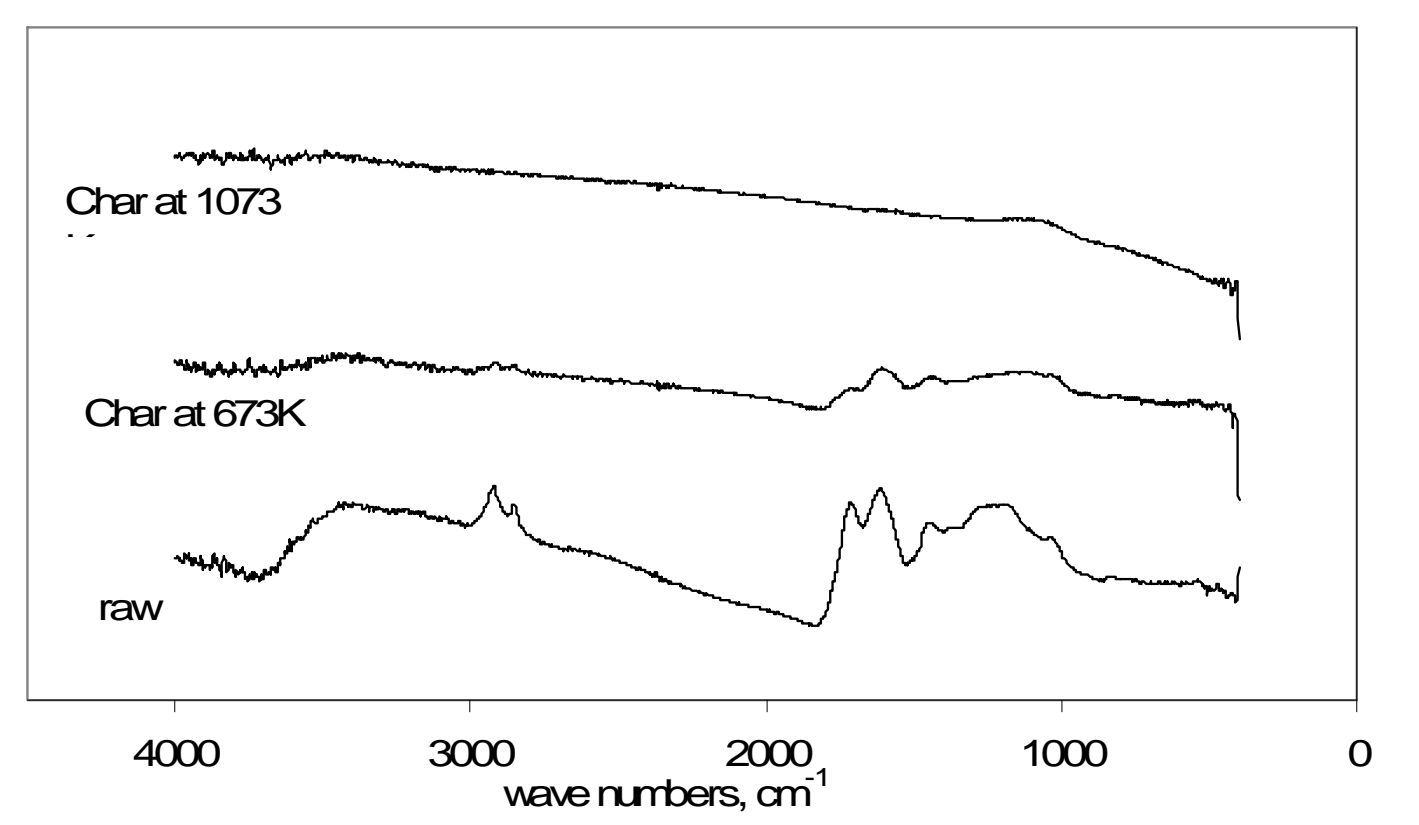

Figure 6.17 FTIR spectra of L4 and its chars at $673 \mathrm{~K}$ and $1073 \mathrm{~K}$

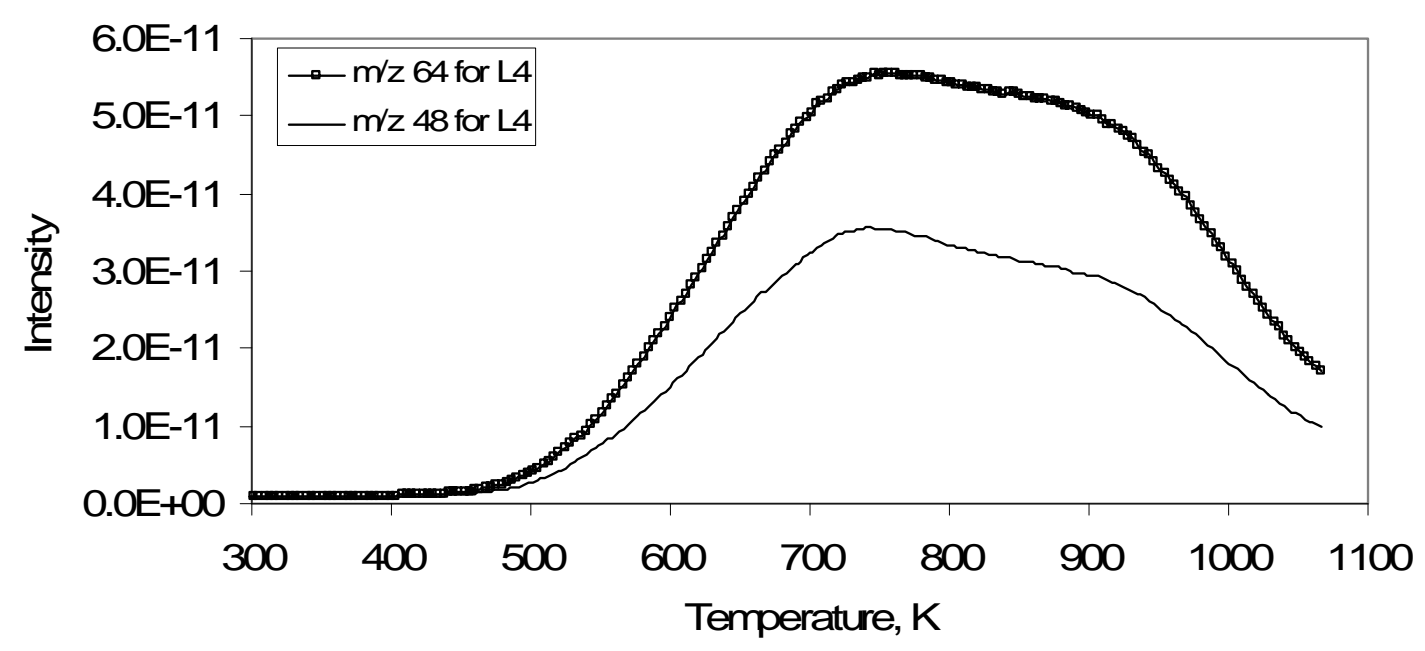

Figure $6.18 \mathrm{SO}_{2}$ evolution during pyrolysis of L4 in a TGA under argon atmosphere 
Due to the volatility of iron sulphates, most of the sulphates are decomposed during pyrolysis. The remaining sulphur from the decomposition of sulphates in the char may also form complex sulphides which hard to be identified by chemical analysis [110].

The shoulder of $\mathrm{SO}_{2}$ released at ca $917 \mathrm{~K}$ could be related to the decomposition of pyrite. Due to high oxygenated functional groups occur in $\mathrm{L} 4$, during pyrolysis $\mathrm{FeS}_{2}$ decomposes to evolve $\mathrm{SO}_{2}$ instead of $\mathrm{H}_{2} \mathrm{~S}$ (see Chapter 5). The decomposition of $\mathrm{FeS}_{2}$ can be described with the following reactions :

$$
\begin{array}{ll}
\mathrm{FeS}_{2(\mathrm{~s})} & \rightarrow \mathrm{FeS}_{\mathrm{x}(\mathrm{s})}+(1-0.5 \mathrm{x}) \mathrm{S}_{2(\mathrm{~g})} \\
2 \mathrm{FeS}_{\mathrm{x}(\mathrm{s})}+(1.5+2 \mathrm{x}) \mathrm{O}_{2(\mathrm{~g})} & \rightarrow \mathrm{Fe}_{2} \mathrm{O}_{3(\mathrm{~s})}+2 \mathrm{xSO}_{2(\mathrm{~g})} \\
2 \mathrm{FeS}_{\mathrm{x}(\mathrm{s})}+(3+2 \mathrm{x}) \mathrm{O}_{2(\mathrm{~g})} & \rightarrow \mathrm{Fe}_{2}\left(\mathrm{SO}_{4}\right)_{3(\mathrm{~s})}+(2 \mathrm{x}-3) \mathrm{SO}_{2(\mathrm{~g})} \\
\mathrm{FeS}_{\mathrm{x}(\mathrm{s})}+(1+\mathrm{x}) \mathrm{O}_{2(\mathrm{~g})} & \rightarrow \mathrm{FeSO}_{4(\mathrm{~s})}+(\mathrm{x}-1) \mathrm{SO}_{2}
\end{array}
$$

Ferric sulphate and ferrous sulphate formed from the decomposition of pyrite is further decomposed following reactions R6.9-R6.10 and R6.12-R6.13, respectively.

Figure 6.17 also shows FTIR spectra of the chars produced at $673 \mathrm{~K}$ and $1073 \mathrm{~K}$. It can be seen that the bands for inorganic sulphate in the regions of $610-680 \mathrm{~cm}^{-1}$ and 1130 $1080 \mathrm{~cm}^{-1}$ are still observable in the $673 \mathrm{~K} \mathrm{~L} 4$ char. These sulphate bands have disappeared in the $1073 \mathrm{~K}$ char. It demonstrates that inorganic sulphates in L4 have totally been decomposed during pyrolysis at $1073 \mathrm{~K}$. 


\subsubsection{Sulphur retention in the char}

The chars produced during pyrolysis of CaSO4+L1, FeSO4+L1 and L4 in the fixed bed reactor have been analysed for their total sulphur using CS analyser and the results are presented in Figure 6.19. The rentention of sulphur in FeSO4+L1 is far less than the retention of sulphur in $\mathrm{CaSO} 4+\mathrm{L} 1$ at a similar pyrolysis temperature.

By observing the evolution of $\mathrm{SO}_{2}$ from the decomposition $\mathrm{CaSO}_{4}$ in $\mathrm{CaSO}_{4}+\mathrm{L} 1$ and $\mathrm{FeSO}_{4}$ in $\mathrm{FeSO}_{4}+\mathrm{L} 1$ at the previous sections, it can be concluded that $\mathrm{CaSO}_{4}$ starts to decompose at ca $623 \mathrm{~K}$, while $\mathrm{FeSO}_{4}$ at around $500 \mathrm{~K}$. It also has been mentioned that calcium sulphate is a stable compound, although it is surrounded by organic matrix, only a slight part of $\mathrm{CaSO}_{4}$ in the sample is decomposed. Whereas, ferrous sulphate is less stable, when it is surrounded by carbon in the lignite, the decomposition is favoured [9]. This observation is in good agreement with the results of sulphur retention in the chars. The $\mathrm{CaSO}_{4}+\mathrm{L} 1$ char produced at $673 \mathrm{~K}$ contains $69.30 \%$ total sulphur and at the highest pyrolysis temperature $(1073 \mathrm{~K}) 64.20 \%$ of total sulphur retained in the char. While for the case of $\mathrm{FeSO}_{4}+\mathrm{L} 1,16.20 \%$ of total sulphur retained in the $673 \mathrm{~K}$ char and only $12.45 \%$ of total sulphur remained in its char produced at $1073 \mathrm{~K}$.

As discussed previously, most of the sulphates present in L4 is iron sulphates which have high volatility and easily to decompose at lower temperature. The sulphur retention in $\mathrm{L} 4$ is higher than in $\mathrm{FeSO}_{4}+\mathrm{L} 1$ at a corresponding pyrolysis temperature. Since L4 also contains pyrite and very low organic sulphur. It can be seen that the L4 char produced at $673 \mathrm{~K}$ contains $58.20 \%$ total sulphur. The total sulphur retention in L4 char decreases significantly with increasing in pyrolysis temperature, at the highest pyrolysis temperature $(1073 \mathrm{~K})$ the total sulphur retention in the char is $23.60 \%$. Most of the sulphur decomposed during pyrolysis of L4 is from the sulphate forms occur in L4. 
Chapter 6

As discussed previously that $\mathrm{FeSO}_{4}$ has higher volatility than $\mathrm{CaSO}_{4}$. To confirm, a comparison between volatile matter and volatile sulphur released during pyrolysis of $\mathrm{FeSO}_{4}+\mathrm{L} 1, \mathrm{CaSO}_{4}+\mathrm{L} 1$ and $\mathrm{L} 4$ is obtained. The results are shown at Figure 6.20. It can be seen that $\mathrm{FeSO}_{4}$ produces more volatile sulphur than $\mathrm{CaSO}_{4}$. The finding is agree with previous studies that a greater amount of volatile sulphur is released during the decomposition of $\mathrm{FeSO}_{4}$ than of $\mathrm{CaSO}_{4}[64,112]$. It also can be observed that a greater amount of volatile sulphur is released during pyrolysis of L4. This confirms that iron sulphates are dominant in L4.

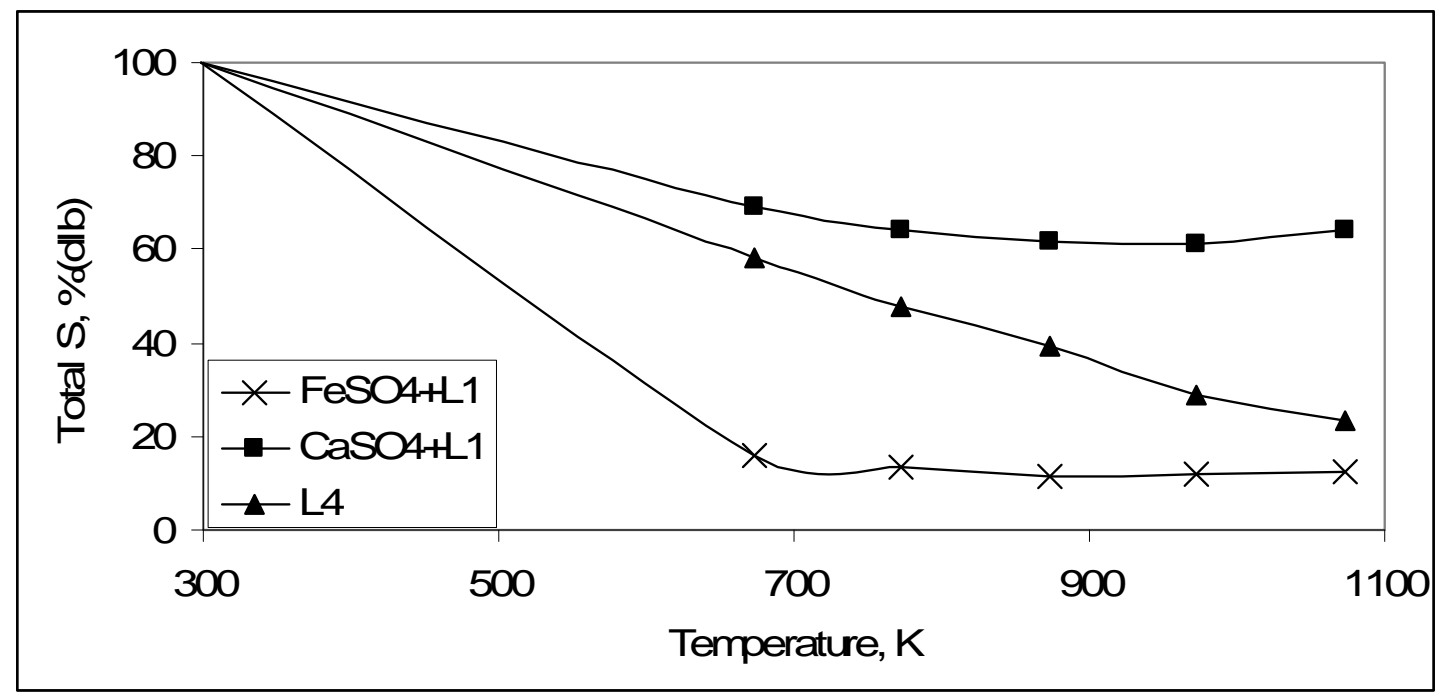

Figure 6.19 Comparison of retention of the total sulphur in chars from $\mathrm{FeSO}_{4}+\mathrm{L} 1, \mathrm{CaSO}_{4}+\mathrm{L} 1$ and $\mathrm{L} 4$ during pyrolysis in the fixed-bed reactor 


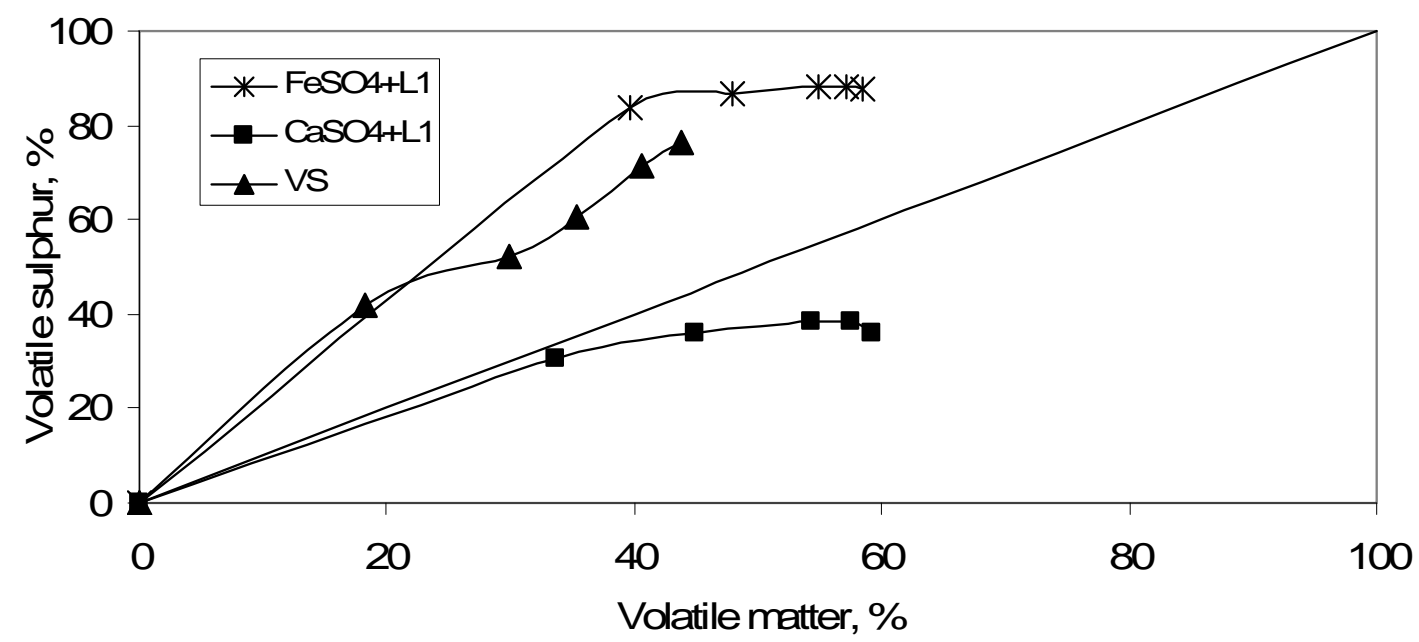

Figure 6.20 Comparison between volatile matter release and volatile sulphur release during pyrolysis of the $\mathrm{FeSO}_{4}+\mathrm{L} 1, \mathrm{CaSO}_{4}+\mathrm{L} 1$ and $\mathrm{L} 4$ during pyrolysis in the fixed-bed reactor

From the previous explanations, it can be seen that $\mathrm{FeSO}_{4}$ has a high volatility. During lignite pyrolysis, the decomposition of iron (II) sulphate occurs as low as $500 \mathrm{~K}$ and most of the sulphate is decomposed during pyrolysis to release $\mathrm{SO}_{2}$. Whereas, $\mathrm{CaSO}_{4}$ is a stable compound, during lignite pyrolysis only a small part of the sulphate is decomposed to evolve $\mathrm{SO}_{2}$, the remaining $\mathrm{CaSO}_{4}$ remains in the similar form in the char.

\subsection{Summary}

The decomposition of pure $\mathrm{CaSO}_{4}$ in a TGA under nitrogen and $\mathrm{N} 2 / \mathrm{CO}_{2}$ mixture is quite similar, $\mathrm{CaSO}_{4}$ decomposes at temperatures between 1400-1700 K. While in air, the decomposition of $\mathrm{CaSO}_{4}$ occurs at temperatures between 1500-1700 K. During pyrolysis of $\mathrm{CaSO}_{4}+\mathrm{L} 1$ in a TGA under nitrogen, $\mathrm{CaSO}_{4}$ starts to decompose at $623 \mathrm{~K}$ to evolve $\mathrm{SO}_{2}$. $\mathrm{CaSO}_{4}$ is a stable compound, only a little part of the $\mathrm{CaSO}_{4}$ decomposes. The undecomposed $\mathrm{CaSO}_{4}$ left in the char is still in the form of $\mathrm{CaSO}_{4}$. 
Chapter 6

During thermal decomposition in TGA under the three different atmosphere (N2, $\mathrm{N} 2 / \mathrm{CO} 2$ mixture and air ), $\mathrm{Fe}_{2}\left(\mathrm{SO}_{4}\right)_{3}$ decomposes between $830-980 \mathrm{~K}$, while $\mathrm{FeSO}_{4}$ decomposes at temperature between 813-953 K. $\mathrm{FeSO}_{4}$ is a more volatile compound, during pyrolysis of $\mathrm{FeSO}_{4}+\mathrm{L} 1$ in a TGA under nitrogen almost all $\mathrm{FeSO}_{4}$ is decomposed to release $\mathrm{SO}_{2}$ in the temperature range of $500-800 \mathrm{~K}$.

Most of the sulphates in L4 are in the form of iron sulphates which have high volatility and the sulphates start to decompose at $500 \mathrm{~K}$. The pyrite in L4 decompose to release $\mathrm{SO}_{2}$, instead of $\mathrm{H}_{2} \mathrm{~S}$, due to the high oxygenated structures in L4. Sulphates has disappeared from the char produced at $1073 \mathrm{~K}$.

During pyrolysis in the fixed bed reactor in nitrogen at $1073 \mathrm{~K}$, only about $36 \%$ of the total sulphur in the $\mathrm{CaSO}_{4}+\mathrm{L} 1$ decomposes. While in the $\mathrm{FeSO}_{4}+\mathrm{L} 1,88 \%$ of the total sulphur decomposes and about $76 \%$ the sulphur in L4 decomposes. It is also confirmed that $\mathrm{FeSO}_{4}+\mathrm{L} 1$ produces more volatile sulphur than $\mathrm{CaSO}_{4}+\mathrm{L} 1$ during pyrolysis. 


\section{CHAPTER 7}

\section{Organic Sulphur Transformation during Lignite Pyrolysis}

\subsection{Introduction}

The amount of organic sulphur in coal is usually between $30-50 \%$ of the total sulphur in the coal [4]. The organic sulphur is originated from sulphur in original vegetations and also from the very complex reaction between organic molecules with inorganic sulphur introduced by sea water during the early diagenesis $[13,37]$.

The exact organic sulphur functional groups occur in coal are very difficult to identify, but a deduction about their forms can be made $[4,31,39]$. Destructive and nondestructive methods have been used to identify organic sulphur functionalities in coal $[4,36,38,40]$. Destructive methods for analysing sulphur functionalities in coal rely on a suitable organic sulphur model compound $[9,38,62]$.

The organic sulphur in coal exists in various functional groups, such as thiolic, sulphidic, disulphidic, sulphoxide and thiophenic (heterocyclic) structures $[4,31,37]$. The amount of thiol and disulphide sulphurs ranges from $60-80 \%$ of total organic sulphur in lignite, $30-50 \%$ of total organic sulphur in bituminous coal and $0 \%$ in anthracite. The lower the coal rank, the higher the labile sulphur contents $[4,5,37,40$, 124]. Table 7.1 summaries some possible thermal decomposition reactions of organic sulphur model compounds in an inert and reducing atmosphere. 
Table 7.1 A summary of possible thermal decomposition reactions of organic sulphur model compounds an inert and reducing atmosphere [5, 47]

\begin{tabular}{|c|c|c|}
\hline Organic S groups & Reactions & Temperature, $\mathrm{K}$ \\
\hline Thiols & $\begin{array}{l}\mathrm{R}-\mathrm{SH} \rightarrow \mathrm{R}-+-\mathrm{SH} \\
\Theta-\mathrm{SH} \rightarrow \Theta-+-\mathrm{SH} \\
\mathrm{R}-\mathrm{SH}+\mathrm{H}_{2} \rightarrow \mathrm{RH}+\mathrm{H}_{2} \mathrm{~S} \\
\Theta-\mathrm{SH}+\mathrm{H}_{2} \rightarrow \Theta-\mathrm{H}+\mathrm{H}_{2} \mathrm{~S}\end{array}$ & $518-623$ \\
\hline Disulphides & $\begin{array}{l}\Theta-\mathrm{S}-\mathrm{S}-\Theta \rightarrow 2 \Theta-+2 \mathrm{~S}- \\
\Theta-\mathrm{S}-\mathrm{S}-\Theta+\mathrm{H}_{2} \rightarrow 2 \Theta-\mathrm{H}+2 \mathrm{H}_{2} \mathrm{~S}\end{array}$ & 673 \\
\hline Sulphides & $\begin{array}{l}\text { R-S-R } \rightarrow \text { R- + S- } \\
\Theta-S-R \rightarrow \Theta-+-S R \\
\Theta-S-\Theta \rightarrow 2 \Theta-+ \text { S- } \\
\text { R-S-R + 2H } \rightarrow 2 \mathrm{RH}+\mathrm{H}_{2} \mathrm{~S} \\
\Theta-S-A r+2 \mathrm{H}_{2} \rightarrow 2 \Theta-\mathrm{H}+\mathrm{H}_{2} \mathrm{~S}\end{array}$ & $723-823$ \\
\hline Sulphoxide / Sulphone & $\begin{array}{l}\Theta-\mathrm{SO}-\Theta \rightarrow 2 \Theta-+ \text { SO- } \\
\Theta-\mathrm{SOO}-\Theta \rightarrow 2 \Theta-+\mathrm{SO}_{2}\end{array}$ & $873-928$ \\
\hline thiophenes & $\overbrace{+\mathrm{H}_{2} \rightarrow \mathrm{C}_{4} \mathrm{H}_{10}+\mathrm{H}_{2} \mathrm{~S}}^{\mathrm{S}}$ & $\geq 923$ \\
\hline
\end{tabular}

$(\Theta$ is an aromatic structure $)$.

Destructive methods have been used to study the organic sulphur model compounds [9, 38, 62]. Mullens et al [38] studied the behaviour of model compounds of organic sulphur immobilised on silica using the atmospheric pressure-temperature programmed pyrolysis coupled with a mass spectrometer (AP-TPR-MS). They clarified the mechanisms behind the low reduction efficiencies of thiophenic and oxidised model compounds to $\mathrm{H}_{2} \mathrm{~S}$. However, to achieve a quantitative sulphur balance needs a more sensitive detection instrument. In addition, even though the sulphur-containing compounds immobilised on silica are stable, the interactions between the inert silica matrix and the sulphur model compounds are different from those between a coal matrix 
Chapter 7

and the sulphur-containing structures. Xu et al [62] studied the behaviour of sulphur model compounds impregnated on a charcoal to simulate coal-like environment in a temperature-programmed decomposition coupled with a mass spectrometer (TPD-MS). They revealed that the key intermediate for volatile sulphur emission is the formation of -S. sulphur radical. Yan et al [9] studied thermal decomposition of six organic sulphur compounds impregnated in a low ash char. They found that $\cdot \mathrm{SH}$ radical was formed during decomposition of organic sulphur. The radical then undergoes secondary reaction with the char to yield gaseous sulphurs such as $\mathrm{H}_{2} \mathrm{~S}, \mathrm{SO}_{2}$ and COS, elemental sulphur and new organic sulphur structures in the char.

Studies of the behaviour of organic sulphur in coal during pyrolysis have been carried out in an atmospheric-pressure temperature-programmed reduction (AP TPR) [47, 63, 125]. It was found that during pyrolysis, the decomposition of organic sulphur depends on the complexity of the organic sulphur. Based on the evolution of gaseous sulphur, they reported that thiols and aliphatic sulphide start to decompose as low as $518 \mathrm{~K}$, aliphatic disulphides and mixed aliphatic-aromatic sulphides decompose at approximately $713 \mathrm{~K}$, aromatic sulphides and disulphides reduction occurs approximately at $883 \mathrm{~K}$, and thiophenes are reduced above $973 \mathrm{~K}$.

In this study two organic sulphur model compounds were namely, naphthalene thiol and benzyl sulphoxide, were used. Thiol is chosen because most of the organic sulphurs in lignite are from the labile organic sulphur, while sulphoxide is chosen since the lignite contains high oxygenated structures. The sulphur compounds were impregnated in a low-sulphur and low-mineral lignite. 


\subsection{Results and Discussion}

The structure of naphthalene thiol and benzyl sulphoxide is presented in Table 7.2. The compounds were impregnated in acid washed L1, the lignite with low mineral and sulphur contents. The impregnated samples were named NT $+\mathrm{L} 1$ for naphthalene thiol impregnated in acid washed L1 and BS+L1 for benzyl sulphoxide impregnated in acid washed L1. The acid washing and impregnation methods have been described in Chapter 3. To compare with inherent organic sulphur in lignite, the lignite sample with high organic sulphur was also used. L3, the lignite with high organic (1.56\%), moderate sulphate content $(0.56 \%)$ and very low pyrite content $(0.06 \%)$ (see Table 4.3 chapter 4$)$ was chosen. To remove sulphate and mineral in L3, acid washing in dilute hydrochloric acid was conducted. The sample was named AW L3. AW L3 has $1.56 \%$ of organic sulphur and $0.16 \%$ of inorganic sulphur.

Table 7.2 Structure and physical properties of sulphur model compounds

\begin{tabular}{|c|c|c|c|c|c|}
\hline \multirow[t]{2}{*}{ Compound } & \multirow{2}{*}{$\begin{array}{l}\text { Molecular } \\
\text { formula }\end{array}$} & \multirow[t]{2}{*}{ Chemical Structure } & \multicolumn{3}{|c|}{ Physical Properties } \\
\hline & & & $\begin{array}{l}\text { MP, } \\
\text { K }\end{array}$ & $\begin{array}{l}\mathrm{BP}, \\
\mathrm{K}\end{array}$ & $\begin{array}{l}\text { MW, } \\
\text { g.gmol }\end{array}$ \\
\hline $\begin{array}{l}\text { Naphthalene } \\
\text { thiol }\end{array}$ & $\mathrm{C}_{10} \mathrm{H}_{8} \mathrm{~S}$ & & $\begin{array}{l}353- \\
354\end{array}$ & $\begin{array}{l}365- \\
367\end{array}$ & 160.24 \\
\hline $\begin{array}{l}\text { Benzyl } \\
\text { sulphoxide }\end{array}$ & $\mathrm{C}_{14} \mathrm{H}_{14} \mathrm{OS}$ & & 403 & $\mathrm{n} / \mathrm{a}$ & 230.33 \\
\hline
\end{tabular}

L3, AW L3, NT+L1, BS+L1 were pyrolysed in a fixed bed reactor in nitrogen atmosphere at a constant flow rate of $1 \mathrm{~L} \cdot \mathrm{min}^{-1}$ at $673 \mathrm{~K}, 773 \mathrm{~K}, 873 \mathrm{~K}, 973 \mathrm{~K}$ and $1073 \mathrm{~K}$. 
Chapter 7

The details of the fixed bed reactor and experimental technique have been described in Chapter 3.

Figure 7.1 shows a comparison of total sulphur retention in chars of L3, AW L3, $\mathrm{NT}+\mathrm{L} 1$ and $\mathrm{BS}+\mathrm{L} 1$, respectively. It can be seen that the total sulphur retention in the chars of all samples decreases with exposure to increasing pyrolysis temperatures. $\mathrm{BS}+\mathrm{L} 1$ and $\mathrm{NT}+\mathrm{L} 1$ experience a major decrease in the retention of total sulphur in the $673 \mathrm{~K}$ char, it has only ca $10 \%$ total sulphur retained in the BS+L1 char and ca $22 \%$ in the $\mathrm{NT}+\mathrm{L} 1$ char, respectively. $\mathrm{NT}+\mathrm{L} 1$ experiences further decrease in the retention of total sulphur up to $873 \mathrm{~K}(9 \%)$. Further increase in the pyrolysis temperature up to $1073 \mathrm{~K}$ did not give significant decrease in the total sulphur retention in the NT+L1 char. While total sulphur in BS+L1 char decreases significantly up to $773 \mathrm{~K}$, at this temperature the total sulphur retained in the char is $7 \%$. At the highest pyrolysis temperature, $1073 \mathrm{~K}$, only $5 \%$ and $7 \%$ total sulphur is retained in $\mathrm{BS}+\mathrm{L} 1$ and $\mathrm{NT}+\mathrm{L} 1$, respectively.

The low retention of total sulphur in $\mathrm{BS}+\mathrm{L} 1$ and $\mathrm{NT}+\mathrm{L} 1$ chars recovered at $673 \mathrm{~K}$ implies that the compounds decompose at a temperature below $673 \mathrm{~K}$. The decomposition of thiol agrees with previous study which reported that thiols decomposes at ca $623 \mathrm{~K}$, and the decomposition of sulphoxide in this experiment is lower than the temperature decomposition reported in previous studies $[5,47]$.

The total sulphur retention in L3 and AW L3 chars are quite similar at $673 \mathrm{~K}$ and $773 \mathrm{~K}$. The total sulphur retention in the BS+L1 and NT+L1 chars yielded at $673 \mathrm{~K}$ and $773 \mathrm{~K}$ are ca $76 \%$ and $61 \%$, respectively. Then above $773 \mathrm{~K} \mathrm{L3}$ has higher total sulphur retention at a corresponding temperature than AW L3. It can be seen from Figure 7.1 that above $873 \mathrm{~K}$ the increase in pyrolysis temperature shows little effect upon the total 
sulphur retention in both L3 and AW L3. At the highest pyrolysis temperature, $1073 \mathrm{~K}$, the total sulphur retentions in AW L3 and L3 chars are 21\% and 31\%, respective. The higher retention of total sulphur in L3 char at a corresponding pyrolysis temperature than in AW L3 is due to interactions of mineral with sulphur in L3 [109]. AW L3 contains less mineral than L3, since most of the sulphur in AW L3 has been washed off during the acid washing $[105,126]$.

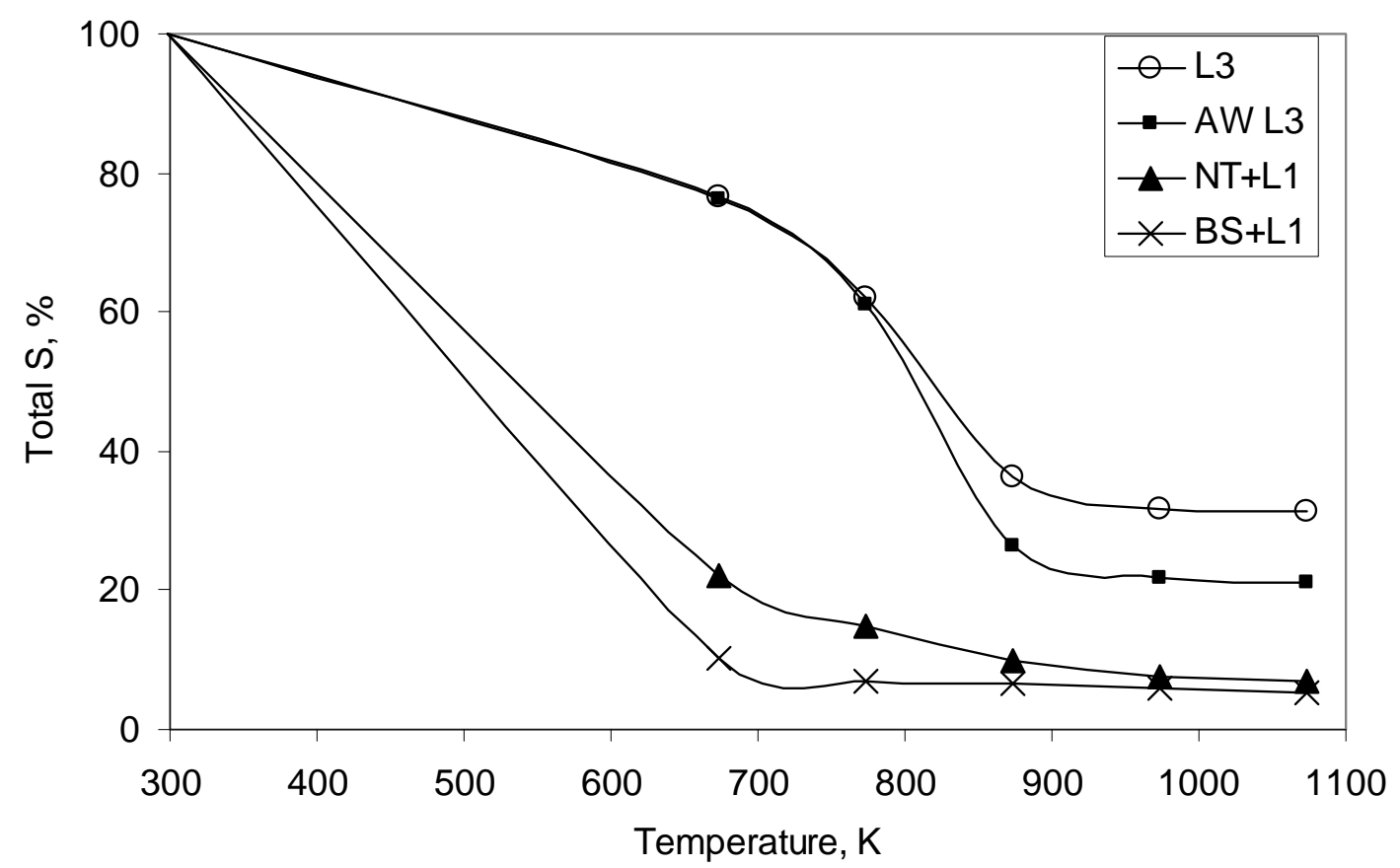

Figure 7.1 Comparison of retention of the total sulphur in chars from L3, AW L3, NT+L1, BS+L1 during pyrolysis in the fixed-bed reactor 


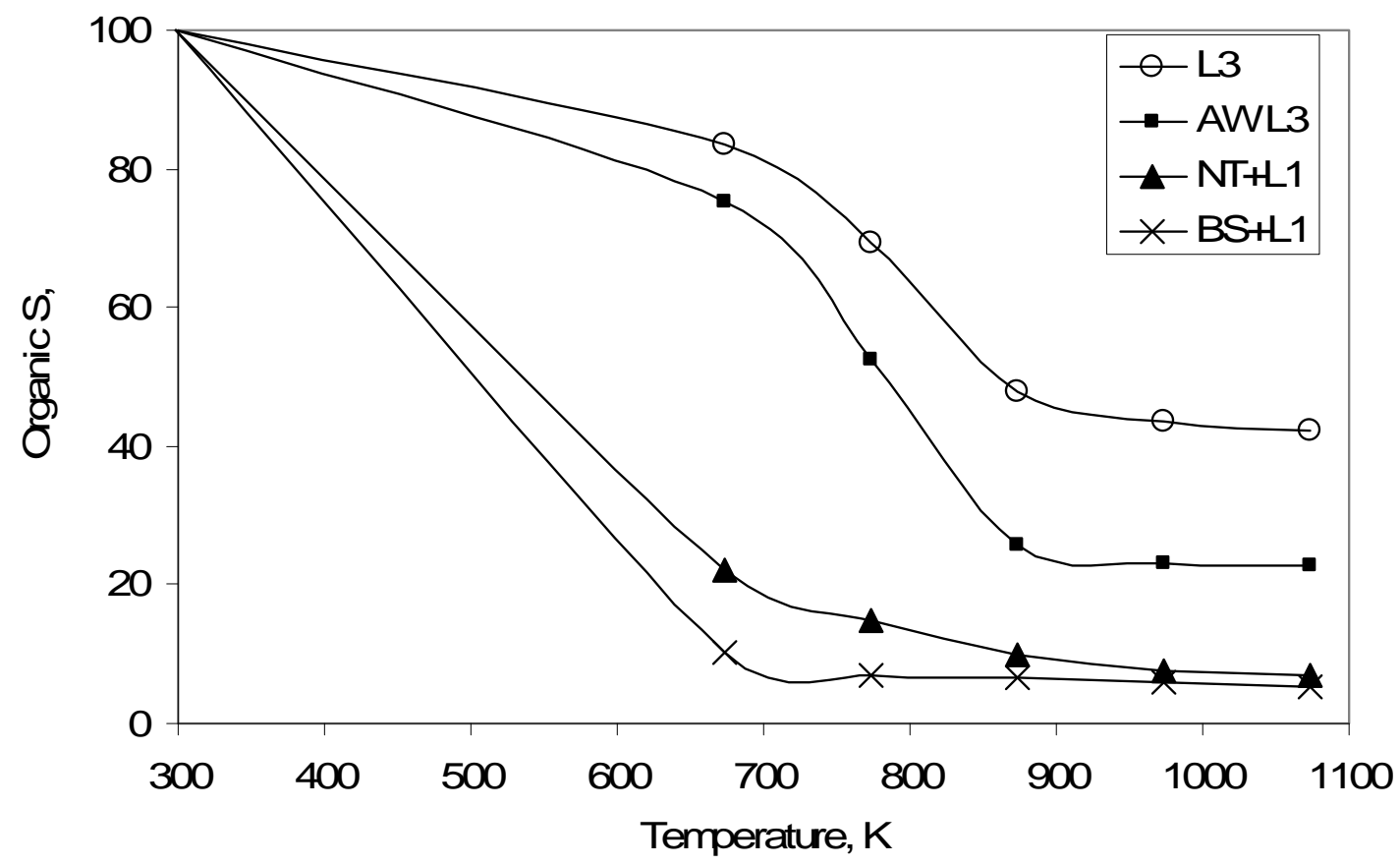

Figure 7.2 Comparison of retention of the organic sulphur in chars from L3, AW L3, NT+L1, BS+L1 during pyrolysis in the fixed-bed reactor

Figure 7.2 compares the retention of organic sulphur in the chars of L3 and AW L3 with the total sulphur retention in chars of $\mathrm{NT}+\mathrm{L} 1$ and $\mathrm{BS}+\mathrm{L} 1$. It is assumed that all the sulphur in $\mathrm{NT}+\mathrm{L} 1$ and $\mathrm{BS}+\mathrm{L} 1$ are organic sulphur. It can be seen that the organic sulphur retention in the L3 and AW L3 chars progressively decreases with rising pyrolysis temperature from $673 \mathrm{~K}$ up to $873 \mathrm{~K}$. The retention of organic sulphur in $\mathrm{NT}+\mathrm{L} 1$ and BS+L1 chars at $673 \mathrm{~K} 83.5 \%$ and $75 \%$, respectively. While in the chars of $\mathrm{NT}+\mathrm{L} 1$ and $\mathrm{BS}+\mathrm{L} 1$ at $873 \mathrm{~K}$, the retention of organic sulphur are $48 \%$ and $26 \%$, respectively. Raising pyrolysis temperatures up to $1073 \mathrm{~K}$ give a minor effect on the decrease in total sulphur retention in the L3 and AW L3 chars. It can be observed that at $1073 \mathrm{~K}$, the retention of organic sulphur in L3 and AW L3 chars are $42 \%$ and $23 \%$, respectively. The retention of organic sulphur in L3 is much higher than in AW L3 at the corresponding pyrolysis temperature. Mineral and inorganic matter has the ability to react with and thus retain organic sulphur in the solid phase during pyrolysis $[2,4,109$, 
Chapter 7

127]. The effect of inorganic matter on the sulphur transformation during lignite pyrolysis will be discussed in Chapter 8 .

By comparing the organic sulphur retention in L3 and AW L3 with the sulphur retention in $\mathrm{NT}+\mathrm{L} 1$ and $\mathrm{BS}+\mathrm{L} 1$, it can be observed that the organic sulphur retention in L3 and AW L3 is somewhat similar to the retention of sulphur in $\mathrm{NT}+\mathrm{L} 1$; the retention of the sulphur decreases significantly up to $873 \mathrm{~K}$ and increasing the pyrolysis temperature up to 1073 only insignificantly affects the reduction of the retention of organic sulphur in the chars. Most of the organic sulphurs have been decomposed below $873 \mathrm{~K}$ and the organic sulphurs are mostly from thiol groups. This confirms that labile organic sulphur is dominant in lignite $[4,5,31,37,124]$.

FTIR and SEM-EDS have been used to qualitatively study the transformation of organic sulphur in solid phases. Figure 7.3 shows FTIR spectra of AW L1, naphthalene thiol, $\mathrm{NT}+\mathrm{L} 1$ and NT+L1 char at 673 and $1073 \mathrm{~K}$. It can be seen that bands are observed at $872,814,772,704$ and $426 \mathrm{~cm}^{-1}$ in $\mathrm{NT}+\mathrm{L} 1(\mathrm{c})$, these bands are not observable in AW L1 (a). Those bands present intensely in naphthalene thiol spectra (b). Thus the bands in the range of $426-872 \mathrm{~cm}^{-1}$ which observed in $\mathrm{NT}+\mathrm{L} 1$ can be associated with the presence of naphthalene thiol. Thiols and thio-substitute compounds have bands in the range of $430-715 \mathrm{~cm}^{-1}$ [122]. Although it is weak, thiol bands is still observable in the $673 \mathrm{~K}$ char of NT+L1 (d). These bands have disappeared from the NT+L1 char yielded at $1073 \mathrm{~K}(\mathrm{e})$. 


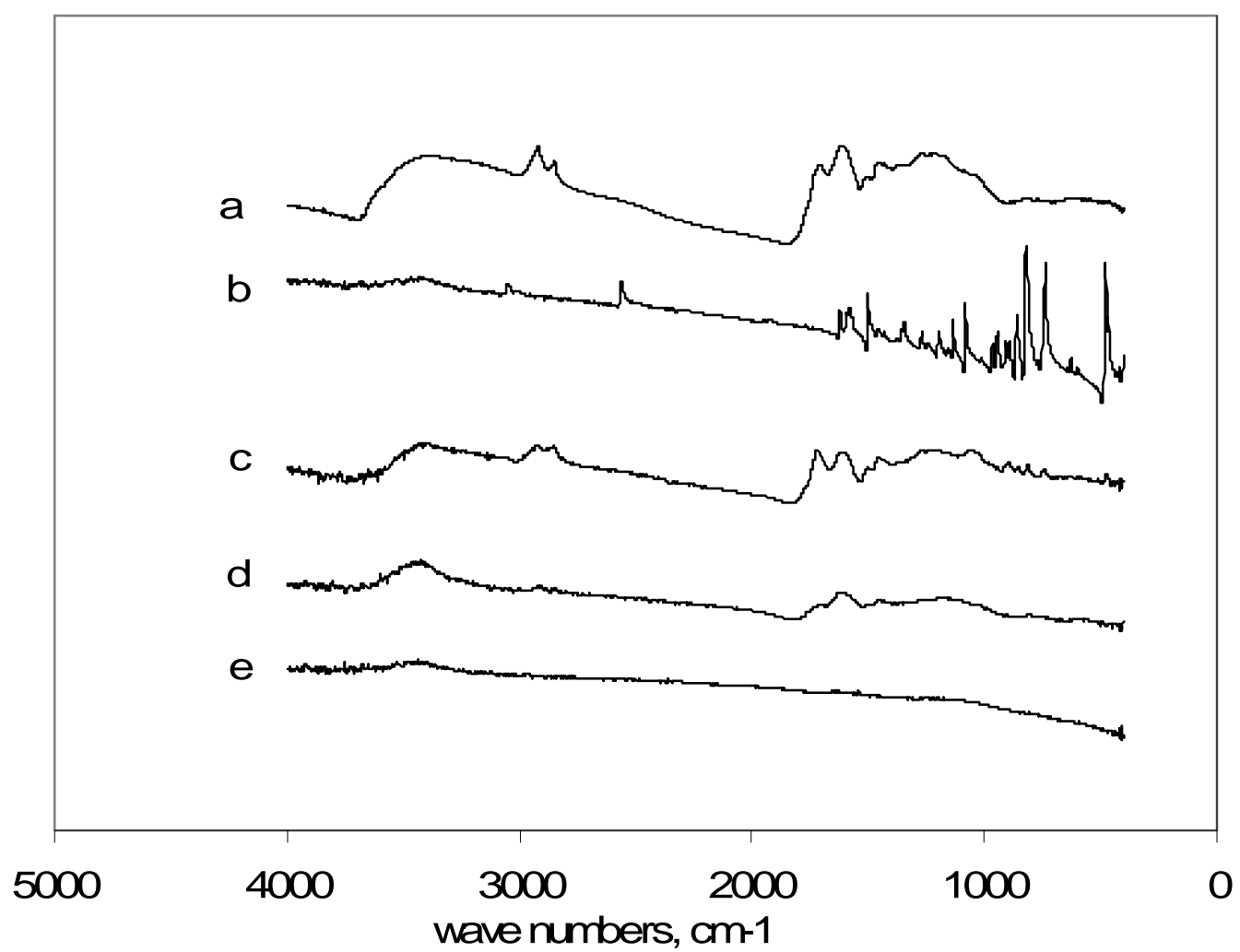

Figure 7.3 FTIR spectra of (a) AW L1, (b) naphthalene thiol, (c) NT+L1, (d) NT+L1 char at $673 \mathrm{~K}$ and (e) NT+L1 char at $1073 \mathrm{~K}$

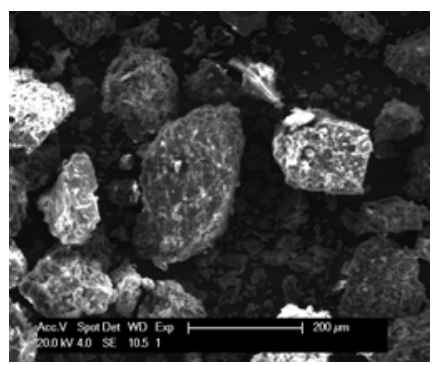

(a)

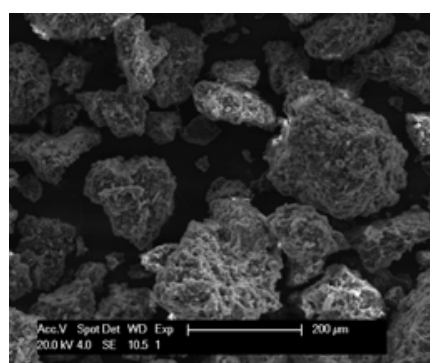

(b)

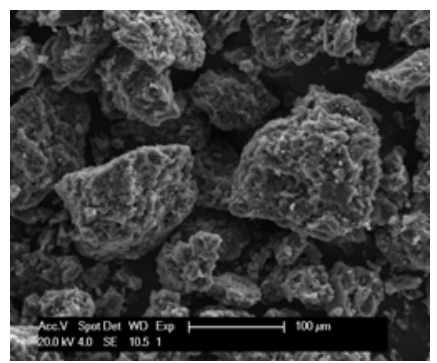

(c)

Figure 7.4 SEM images of (a) NT+L1, (b) NT+L1 char at $673 \mathrm{~K}$ and (c) NT+L1 char at $1073 \mathrm{~K}$

Figure 7.4 shows the SEM images of NT+L1 and its chars at 673 and $1073 \mathrm{~K}$. It is observed that the chars are more porous than unpyrolysed $\mathrm{NT}+\mathrm{L} 1$, the porosity has also increased with increasing pyrolysis temperature. It was reported that organic sulphur decomposition begins with the formation of $-\mathrm{SH}$ radical [9], the formation of the radicals is favour with increasing in the porosity of the chars. 

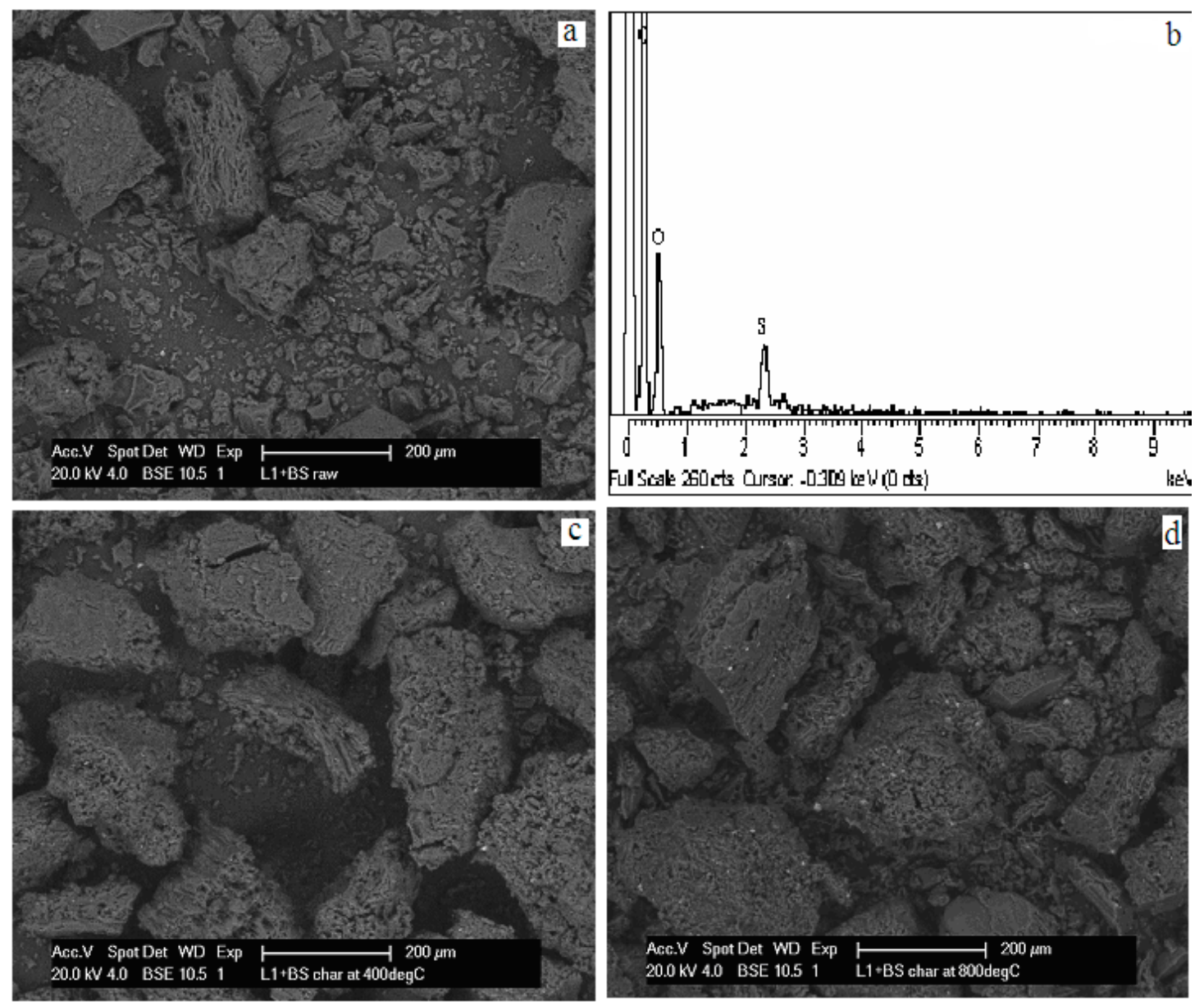

Figure 7.5 SEM images of (a) BS+L1, (c) BS +L1 char at 673K,(d) BS +L1 char at $1073 \mathrm{~K}$ and (b) EDS spectra of BS+L1

Figure 7.5 presents the SEM image of BS+L1 and its EDS spectra as well as SEM images of its chars at $673 \mathrm{~K}$ and $1073 \mathrm{~K}$. Similar to the case for NT+L1, the pyrolysis temperature affects the porosity of the char. It is observable from SEM micrographs that the porosity of the char increases with increasing in pyrolysis temperature. Higher porosity and higher temperature contribute positively to the decomposition of benzyl sulphoxide in $\mathrm{BS}+\mathrm{L} 1$ evolving gaseous sulphur. 

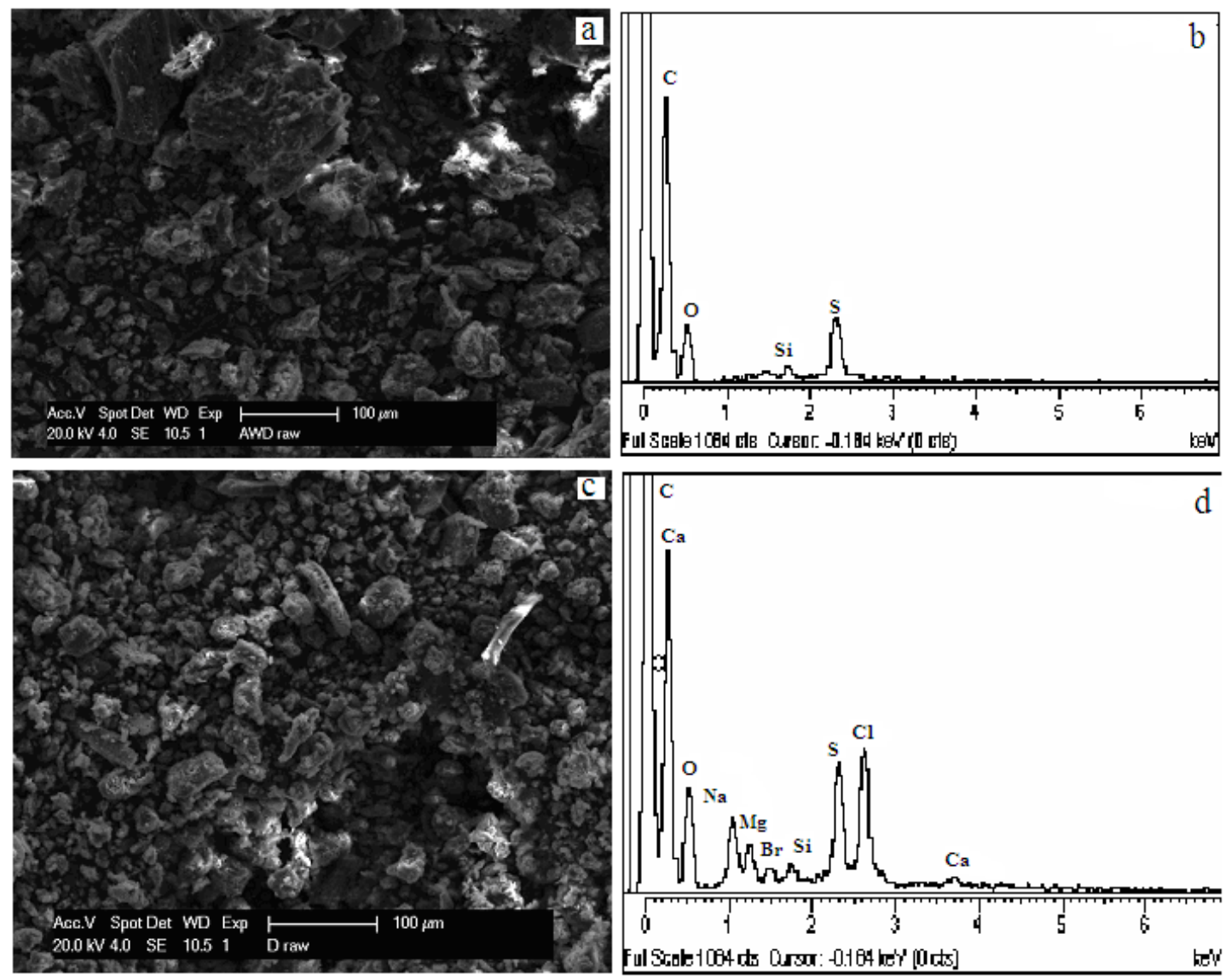

Figure 7.6 SEM images and EDS spectra of AW L3 ( $a$ and b) and L3 (c and d)

Figure 7.6 shows the SEM images of L3 and AW L3 as well as their EDS spectra. Acid washing in dilute hydrochloric acid dissolves some of mineral and inorganic matter present in $\mathrm{L} 3$. It can be seen that $\mathrm{Br}, \mathrm{Ca}, \mathrm{Cl}, \mathrm{Mg}$ and $\mathrm{Na}$ which are detected by EDS in L3 are disappeared in AW L3. SEM micrographs show that the acid washing has not significantly changed the texture of lignite.

The decrease in the inorganic matter contents in acid washed L3 can also be seen in Figure 7.7 showing FTIR spectra of L3, AW L3 and AW L3 chars. Mineral and inorganic bands observed in the range of $400-600 \mathrm{~cm}^{-1}$ and $3600-4000 \mathrm{~cm}^{-1}[88,89$, 109]. The mineral and inorganic bands are more intense in L3 (Figure 7.7 a) than in AW L3 (Figure 7.7 b). Thiols and thio-substitute compounds bands in the range of 430-715 
$\mathrm{cm}^{-1}[122]$ as well as C-S stretching vibration in the range of $570-705 \mathrm{~cm}^{-1}[123,128]$ are very weak in both L3 and AW L3.

Oxidised organic sulphur compounds or sulphur-oxy compounds are formed based on the type of organic sulphur and the oxidising conditions, they may present in the form of sulphoxides, sulphone, sulphonic acid, sulphinic acid or sulphenic acid. Whatever the oxidised organic sulphur forms, they have $-\mathrm{S}=\mathrm{O}$ stretching vibration [123]. Band assignments of sulphur-oxy compounds are presented in Table 7.3. Sulphoxides, sulphone, sulphonate and sulphates present in AW L3 and L3, since broad bands in the range of 1020-1420, the oxy-sulphur regions, are observed in both samples. The broad bands in the range of 1000-1800 are also the indication that AW L3 and L3 have high oxygenated functional groups [18].

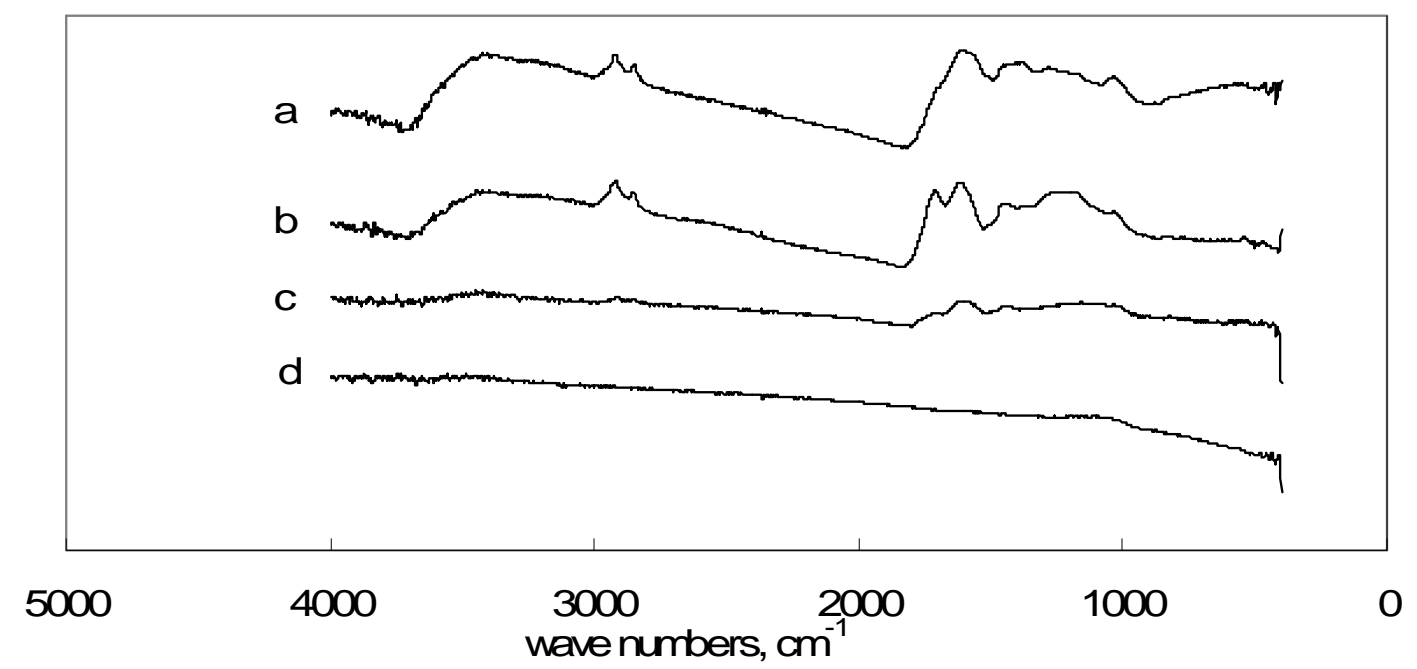

Figure 7.7 FTIR spectra of (a) L3, (b) AW L3, (c) AW L3 char at $673 \mathrm{~K}$ and (d) AW L3 char at $1073 \mathrm{~K}$

It can be seen from Figure 7.7 that the bands in the range of $1020-1420 \mathrm{~cm}^{-1}$ in AW L3 char yielded at $673 \mathrm{~K}$ are weaker than in AW L3. This suggests that organic sulphur has been decomposed at $673 \mathrm{~K}$. By increasing pyrolysis temperature to $1073 \mathrm{~K}$, it is clearly shown in Figure 7.7 that less organic sulphur is retained in the char. This agrees with the 
finding of organic sulphur retention in AW L3 char, at $1073 \mathrm{~K}$ only $23 \%$ of organic sulphur is retained in the char.

Table 7.3 Band assignments of sulphur-oxy compounds [122, 129]

\begin{tabular}{|l|l|}
\hline Bands, $\mathrm{cm}^{-1}$ & Functional group \\
\hline $1070-1030$ & Sulphoxides \\
\hline $1350-1300 / 1160-1120$ & Sulphone \\
\hline $1365-1340 / 1200-1100$ & Sulphonates \\
\hline $1420-1370 / 1200-1180$ & Organic sulphates \\
\hline
\end{tabular}

The evolution of gases during lignite pyrolysis is also studied using a thermogravimetric analyser coupled with a mass spectrometer (TGA-MS). AW L3 which is assumed to only contain organic sulphur based on the SEM-EDS and FTIR data (Figure 7.6-7.7) is chosen to be run in a TGA-MS from room temperature to $1700 \mathrm{~K}$ at a heating rate of 10 K. $\min ^{-1}$. To avoid similar $\mathrm{m} / \mathrm{z}$ signal of $\mathrm{CO}$ and $\mathrm{N}_{2}$, argon was used as the gas atmosphere at a flow rate of $100 \mathrm{ml} \cdot \mathrm{min}^{-1}$. The sulphur gases evolved as well as other gases were monitored during pyrolysis.

During pyrolysis, the dominant sulphur gas products from the decomposition of organic sulphur is hydrogen sulphide $[127,130]$. However, $\mathrm{m} / \mathrm{z} 33$ and $\mathrm{m} / \mathrm{z} 34$, the $\mathrm{m} / \mathrm{z}$ values for the $\mathrm{H}_{2} \mathrm{~S}$ gas, is not detected in the MS during pyrolysis, instead the sulphur gas evolves as sulphur dioxide which has $\mathrm{m} / \mathrm{z}$ values of $48(\mathrm{SO})$ and $64\left(\mathrm{SO}_{2}\right)$. Figure 7.8 presents the evolution of sulphur dioxide during pyrolysis of AW L3. 


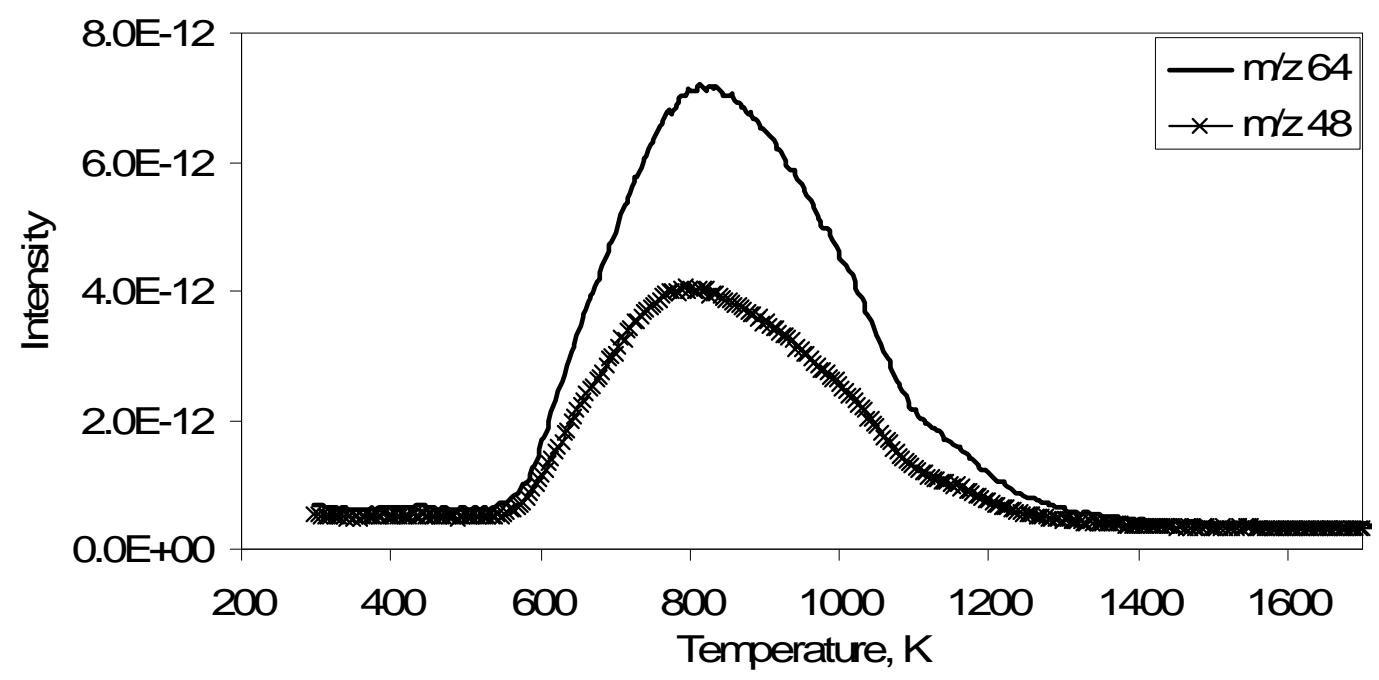

Figure 7.8 Evolution of $\mathrm{SO}_{2}(\mathrm{~m} / \mathrm{z} 64$ and $\mathrm{m} / \mathrm{z} 48)$ during pyrolysis of $\mathrm{AW} \mathrm{L} 3$ in a TGA-MS

The evolution of $\mathrm{SO}_{2}$ begins as low as ca $570 \mathrm{~K}$ and peaks at ca $820 \mathrm{~K}$. There is no more $\mathrm{SO}_{2}$ released from AW L3 at approximately $1300 \mathrm{~K}$. The evolution of gaseous sulphur at a low temperature suggests that the forms of organic sulphur in AW L3 are the labile organic sulphur functional forms. It has been discussed in previous paragraphs that the labile organic sulphur compounds, naphthalene thiol and benzene sulphoxide, impregnated in L3 decomposes at temperatures lower than $673 \mathrm{~K}$.

The evolution of $\mathrm{SO}_{2}$ during pyrolysis of AW L3 instead of $\mathrm{H}_{2} \mathrm{~S}$ also confirms that sulphur-oxy compounds as detected by FTIR are dominant in AW L3. During the pyrolysis $-\mathrm{SO}=$ in sulphur-oxy compounds decomposes to evolve sulphur dioxide. Other organic sulphur which may present in AW L3 decomposes to form - $\mathrm{SH}$ radicals [9]. Some of the $-\mathrm{SH}$ radicals incorporate in the char, while others undergo complex reactions with organic matrix of the lignite which contains high oxygenated functionals as confirmed by FTIR spectra (Figure 7.7) to produce $\mathrm{SO}_{2}$ instead of $\mathrm{H}_{2} \mathrm{~S}$. 


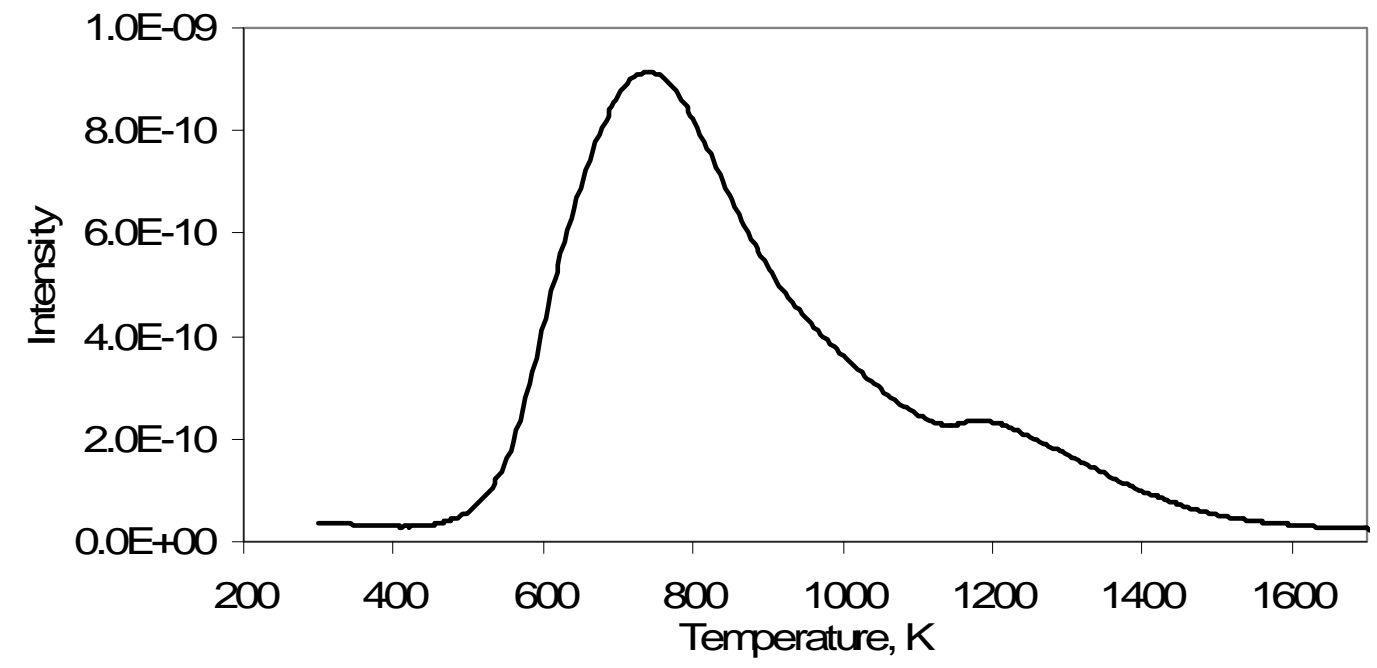

Figure 7.9 Evolution of $\mathrm{CO}_{2}(\mathrm{~m} / \mathrm{z} 44)$ during pyrolysis of AW L3 in a TGA-MS

The complex reactions between $-\mathrm{SH}$ radicals and the char induce the formation of $\mathrm{H}_{2} \mathrm{O}$ and $\mathrm{CO}_{2}$ [9]. The evolutions of $\mathrm{CO}_{2}$ and $\mathrm{H}_{2} \mathrm{O}$ during pyrolysis of $\mathrm{AW} \mathrm{L} 3$ are presented in Figure 7.9 and Figure 7.10, respectively. The released of $\mathrm{CO}_{2}$ starts at ca $500 \mathrm{~K}$ and peaks at ca $750 \mathrm{~K}$. There is a shoulder between 1150-1270 K. Apart from the effect of $\mathrm{SH}$ radicals reaction with the char organic matrix, the evolution of $\mathrm{CO}_{2}$ at low temperature is from the decomposition of aliphatic and aromatic carboxyl groups, while at high temperature $\mathrm{CO}_{2}$ evolution is from the decomposition of more stable ether groups, quinones and oxygen-bearing heterocycles [131].

The evolution of water starts from the beginning of the process until ca $1300 \mathrm{~K}$. The water evolved below $473 \mathrm{~K}$ is mostly from decomposition the physically adsorbed water, while above $473 \mathrm{~K}$ water released is due to the decomposition of chemically combined or formed water [132]. The water evolution above $473 \mathrm{~K}$ also resulted from the complex reactions between - $\mathrm{SH}$ radicals with the char. 


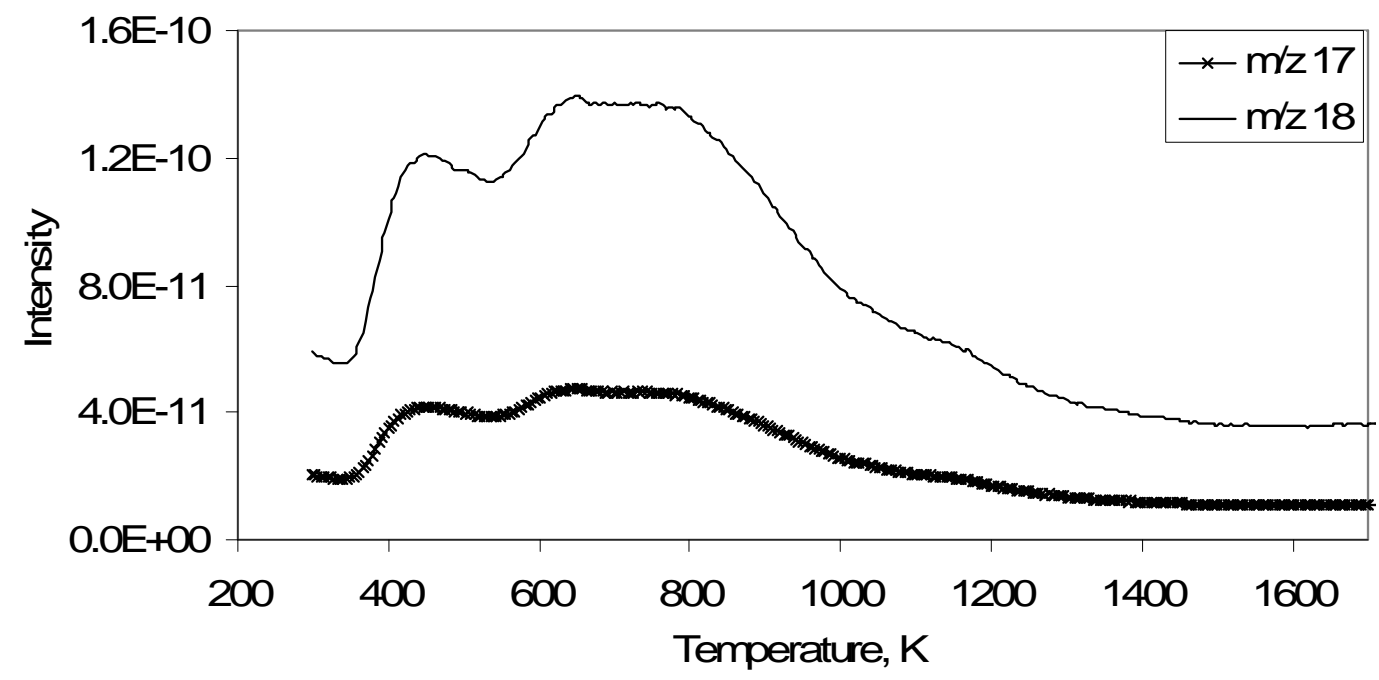

Figure 7.10 Evolution of $\mathrm{H}_{2} \mathrm{O}(\mathrm{m} / \mathrm{z} 17$ and $\mathrm{m} / \mathrm{z}$ 18) during pyrolysis of AW L3 in a TGA-MS

Besides the dominant species $\left(\mathrm{SO}_{2}, \mathrm{CO}_{2}\right.$ and $\left.\mathrm{H}_{2} \mathrm{O}\right)$, fragments with the $\mathrm{m} / \mathrm{z}$ values of 35 , $36,37,51,52,56,58,60$ and 76 are also detected in the MS spectra. Interestingly, hydrogen $(\mathrm{m} / \mathrm{z} 2)$ and $\mathrm{CO}(\mathrm{m} / \mathrm{z} 28)$ which usually release during coal/lignite pyrolysis are not found in the MS spectra. The high oxygenated structure of AW L3 is the reason for the lack of $\mathrm{H}_{2}$ and $\mathrm{CO}$ in the gases, instead $\mathrm{H}_{2} \mathrm{O}$ and $\mathrm{CO}_{2}$ are found to be dominant gases evolved during pyrolysis. The lack of $\mathrm{H}_{2} \mathrm{~S}$ in the gaseous sulphur is also due to the high oxygenated functional groups in AW L3. The un-oxidised organic sulphur compounds which may present in AW L3 decomposes to form - SH radicals which undergo complex reactions with the organic matrix of the char to yield $\mathrm{SO}_{2}$ rather than $\mathrm{H}_{2} \mathrm{~S}$. While other $\mathrm{SO}_{2}$ in the sulphur gas phase is due to the decomposition of sulphuroxy organic compounds which present in AW L3. 


\subsection{Summary}

Both naphthalene thiol in $\mathrm{NT}+\mathrm{L} 1$ and benzyl sulphoxide in $\mathrm{BS}+\mathrm{L} 1$ decompose below $673 \mathrm{~K}$. The retention of total sulphur in NT+L1 char decreases significantly from $673 \mathrm{~K}$ to $873 \mathrm{~K}$, increasing pyrolysis temperature to $1073 \mathrm{~K}$ only gives minor reduction on the total sulphur retention in $\mathrm{NT}+\mathrm{L} 1$ char. While the retention of total sulphur in $\mathrm{BS}+\mathrm{L} 1$ reduces significantly from $673 \mathrm{~K}$ to $773 \mathrm{~K}$, above $773 \mathrm{~K}$ the total sulphur retention does not reduce significantly.

The retention of total sulphur in L3 and AW L3 reduces significantly from $673 \mathrm{~K}$ to 873 $\mathrm{K}$. Above $873 \mathrm{~K}$ there is not much decrease in the total sulphur retention in L3 and AW L3 chars with increasing pyrolysis temperatures. Above $773 \mathrm{~K}$, the total sample retention in L3 is higher than in AW L3, this is due to the interaction between inorganic matter presents in L3 with the sulphur retaining sulphur in the solid phase.

The retention of organic sulphur in L3 and AW L3 is quite similar to the retention of sulphur in $\mathrm{BS}+\mathrm{L} 1$. The organic sulphur retention decreases significantly from 673 to $873 \mathrm{~K}$ and rising the pyrolysis temperature to $1073 \mathrm{~K}$ only give minor effect to the decrease of the organic sulphur in the L3 and AW L3 chars. The retention of organic sulphur in L3 is higher than in AW L3 at the corresponding temperatures due to the interaction between organic sulphur with inorganic matter. Inorganic matter helps to retain organic sulphur in the chars.

FTIR study shows that naphthalene thiol is still observable in the NT+L1 char yielded at $673 \mathrm{~K}$, however at $1073 \mathrm{~K}$ the naphthalene thiol bands has disappeared. SEM micrographs of $\mathrm{NT}+\mathrm{L} 1$ and its chars as well as $\mathrm{BS}+\mathrm{L} 1$ and its chars show that the porosity increases by increasing pyrolysis temperatures. This contributes to the decomposition of naphthalene thiol and benzyl sulphoxide. 
SEM EDS shows that acid washing process dissolves most of the inorganic matter presents in L3. FTIR study shows that sulphur-oxy compounds presents in both L3 and AW L3. The sulphur-oxy compounds are still observable in the char produced at $673 \mathrm{~K}$, but have disappeared in $1073 \mathrm{~K}$ char.

Sulphur dioxide is the only gaseous sulphur produced during pyrolysis of AW L3, $\mathrm{SO}_{2}$ evolution is from the decomposition of oxygenated organic sulphur and also from the decomposition of other labile organic sulphur which presents in AW L3. The evolution of $\mathrm{SO}_{2}$ induces the released of $\mathrm{CO}_{2}$ and $\mathrm{H}_{2} \mathrm{O}$ which are found to be significant during this lignite pyrolysis.

The transformation of two organic sulphur model compounds, namely, naphthalene thiol and benzyl sulphoxide which are impregnated in a low-sulphur and low-inorganic lignite has been thoroughly discussed. The transformation of inherent organic sulphur in lignite has also been discussed in this chapter. In the two previous chapters, Chapter 5 and 6 , the transformations of pyrite and sulphate during lignite have also been discussed in detail. As discussed in Chapter 4, except for L1, the lignites contain extremely high mineral and inorganic matter. To give a better understanding on the effect of inorganic on the sulphur transformation during lignite pyrolysis the following chapter, Chapter 8 , will be devoted to discuss the effect of inherent and added inorganic matter on the sulphur transformation during lignite pyrolysis. 


\section{CHAPTER 8}

\section{The effect of Inorganic Matter on Sulphur}

\section{Transformation during Lignite Pyrolysis}

\subsection{Introduction}

Inorganic matter in coal is all elements present except organically derived or bound C, $\mathrm{H}, \mathrm{N}, \mathrm{O}$, and $\mathrm{S}[22]$. The presence of inorganic matter in coal limits the optimisation of the performance of coal utilisation processes, since inorganic matter is mostly associated with problems encountered during coal utilisations, such as ash fouling, deposition and slagging and particulate matter formation [20, 105, 133-135].

The retention of both organic and inorganic forms of sulphur in the char during coal pyrolysis is influenced by inorganic matter present in the coal. Alkali and alkali earth metals react with sulphur to form low melting-point eutectics which cause slagging, fouling, agglomeration and defluidisation during coal utilisation $[10,115,117]$. Therefore, it is necessary to study the effect of inorganic matter on the sulphur transformations.

This chapter will discuss the effect of inherent and added inorganic species on the sulphur transformation during lignite pyrolysis in a fixed bed reactor. The reactor and pyrolysis technique used have been described in Chapter 3. As discussed in Chapter 4, the lignites, except L1, contain extremely high inorganic matter. The amount of sulphur, organic and inorganic sulphur, in the lignites (except L1) is also considerable. In this study the effect of inherent inorganic matter on the transformation of organic and inorganic sulphur during lignite pyrolysis will be studied using L2 and L3 samples. L2 
Chapter 8

is the lignite with high inorganic matter, high organic sulphur and high inorganic sulphur, while L3 is the lignite with high inorganic matter and high organic sulphur but low in inorganic sulphur. The influence of inherent inorganic matter on the transformation of inorganic and organic sulphur was investigated by comparing the sulphur transformations in the raw lignites and acid washed lignite, the latter removes inherent organics.

The role of sodium and aluminium-silica in sulphur transformations is further examined by adding sodium and kaolinite $\left(\mathrm{Al}_{2} \mathrm{Si}_{2} \mathrm{O}_{5}(\mathrm{OH})_{4}\right)$ to the acid washed L3. The method of adding the inorganic species in the acid washed L3 is described in Chapter 3. To confirm the sodium and kaolinite were bonded in AW L3, scanning electron microscope (SEM) equipped with an energy-dispersive X-ray spectroscopy (EDS) was used.

\subsection{Results and Discussion}

\subsubsection{Effect of inherent inorganic}

L2 and L3 contain high moisture, volatile matter, oxygen and ash as shown in Table 4.1. L3 contains also high organic sulphur (1.74\%), high pyrite (1.61\%) and high sulphate (1.27\%), whereas the sulphur forms in L3 are dominated by organic sulphur (1.56\%) but is very low in pyrite(ca. $0.06 \%)$ and sulphate $(0.56 \%)$ as can be seen in Table 4.3 . Sodium is the dominant inorganic matter in L2 and L3 as shown in Table 4.7.

To study the effect of inherent inorganic matter on the sulphur transformation during lignite pyrolysis, L2 and L3 as well as their acid washed samples, AW L2 for acid washed L2 and AW L3 for acid wshaed L3, were pyrolysed in a fixed bed quartz reactor in nitrogen at a constant flow rate of $11 . \mathrm{min}^{-1}$ at $673 \mathrm{k}, 773 \mathrm{~K}, 873 \mathrm{~K}, 973 \mathrm{~K}$, and 1073 K. The sulphur retained in the chars were analysed for total, organic and inorganic 
Chapter 8

sulphur using CS analyser. The analysis method is described in detail in Chapter 3. The retentions of the sulphur, including total sulphur, organic sulphur, and inorganic sulphur, during pyrolysis of L2, AW L2, L3 and AW L3 in a fixed bed reactor are presented in Figures 8.1-8.6.

It can be observed from Figure 8.1 that the retention of the total and organic sulphur in L2 slightly decreases between $673-773 \mathrm{~K}$. Between $773-873 \mathrm{~K}$ the retention of the total and organic sulphur in L2 experiences a significant decrease but levels off and stays almost constant above $873 \mathrm{~K}$, while the retention of inorganic sulphur in L2 decreases significantly from $673 \mathrm{~K}$ to $1073 \mathrm{~K}$.

The retention of the total, organic and inorganic sulphur in AW L2 is present in Figure 8.2. It can be seen that the retention of total, organic and inorganic sulphur is reduced considerably between 673-973 K and becomes almost constant above $973 \mathrm{~K}$.

Figure 8.3 compares the retention of organic sulphur in L2 and AW L2. It can be observed that $99 \%$ of organic sulphur is retained in $\mathrm{L} 2$ char at $673 \mathrm{~K}$, reduced significantly to $71 \%$ at $873 \mathrm{~K}$ and at $1073 \mathrm{~K}$ approximately $64 \%$ of the organic sulphur is still present in the char. While in AW L2 char at $673 \mathrm{~K} 93 \%$ of the organic sulphur is retained. The retention is then decreased significantly to $40 \%$ at $973 \mathrm{~K}$ and at $1073 \mathrm{~K}$ there is only $35 \%$ organic sulphur retained in the char. The retention of organic sulphur in L2 is much higher than in its acid washed sample, AW L2. This is due to the reactions of inherent inorganic matter present in L2 with the sulphur-bearing compounds, retaining sulphur in the char $[2,4]$. This reaction is believed to occur between the inherent inorganic matter and the $\mathrm{SH}^{*}$ radicals produced from the decomposition of the organic sulphur [9]. The inherent inorganic matter in L2 is dominated by sodium, followed by silica and magnesium, whereas calcium only 
Chapter 8

presents in a small quantity (Table 4.7 Chapter 4). Previous studies of the effect of $\mathrm{Na}$ and $\mathrm{Ca}$ on the sulphur transformation during pyrolysis of Bowmans coal showed that sodium and calcium began to retain sulphur at around 773 and $873 \mathrm{~K}$ [2], respectively, supporting the above observation made in this study.

Figure 8.4 displays a comparison of inorganic sulphur retention in the chars of L2 and AW L2. The inorganic sulphur in L2 consists of pyrite (1.61\%) and sulphate (1.74\%) as can be observed from Table 4.1 Chapter 4. The figure shows that the retention of inorganic sulphur in L2 is much higher than in AW L2. After acid washing the amount of sulphate in AW L2 reduces significantly, thus most of the inorganic sulphur in AW L2 is in the form of pyrite. In Chapter 5, it was revealed that in an inert atmosphere pyrite decomposes to release $\mathrm{H}_{2} \mathrm{~S}$ [51]. Hydrogen sulphide may react with alkaline matter in the char to produce inorganic sulphide sulphur $[2,136]$. The inorganic matter in the lignite has the ability to retain the products of pyrite decomposition in the char as inorganic sulphide sulphur.

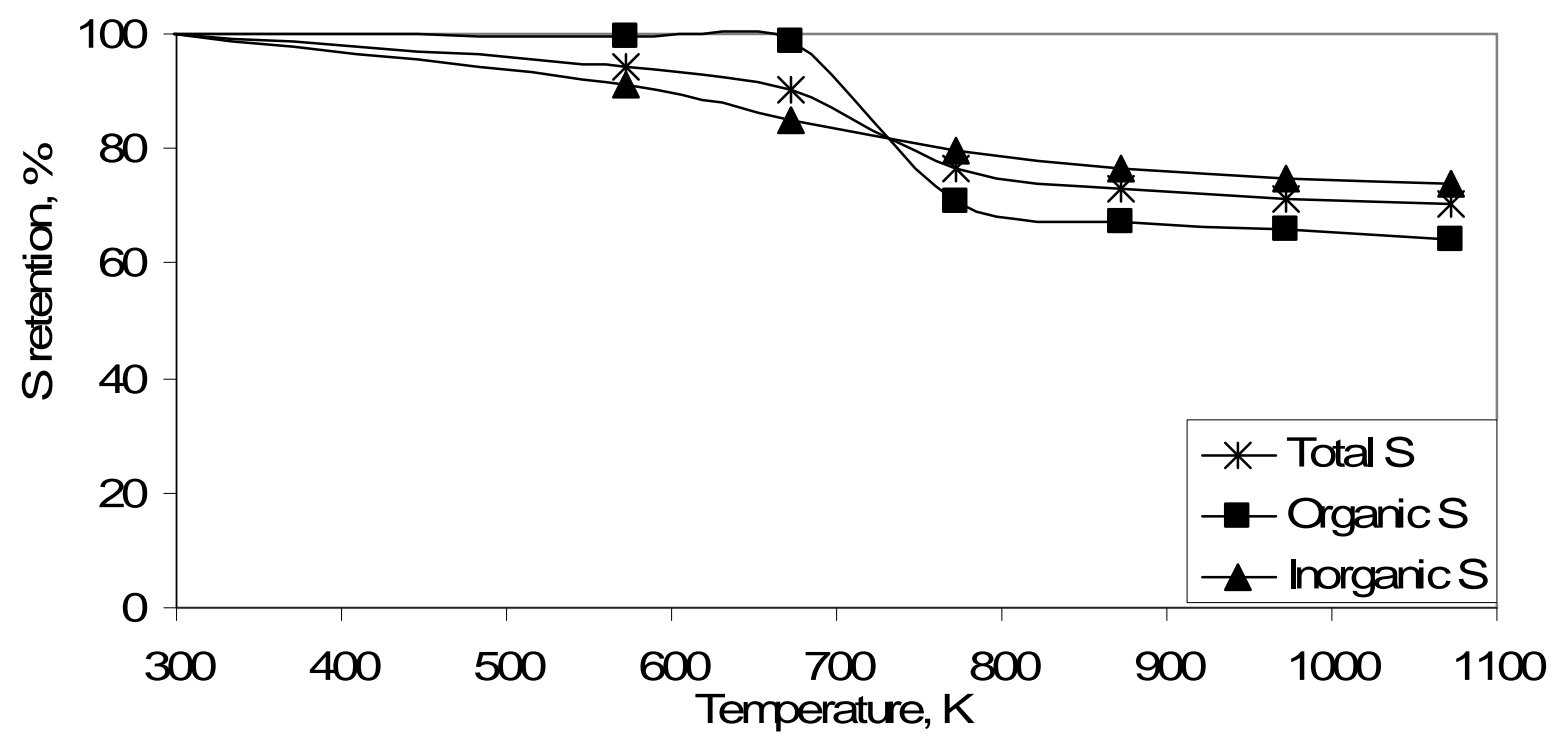

Figure 8.1 The retention of total sulphur, organic sulphur and inorganic sulphur in the $\mathrm{L} 2$ chars during fixed bed pyrolysis 


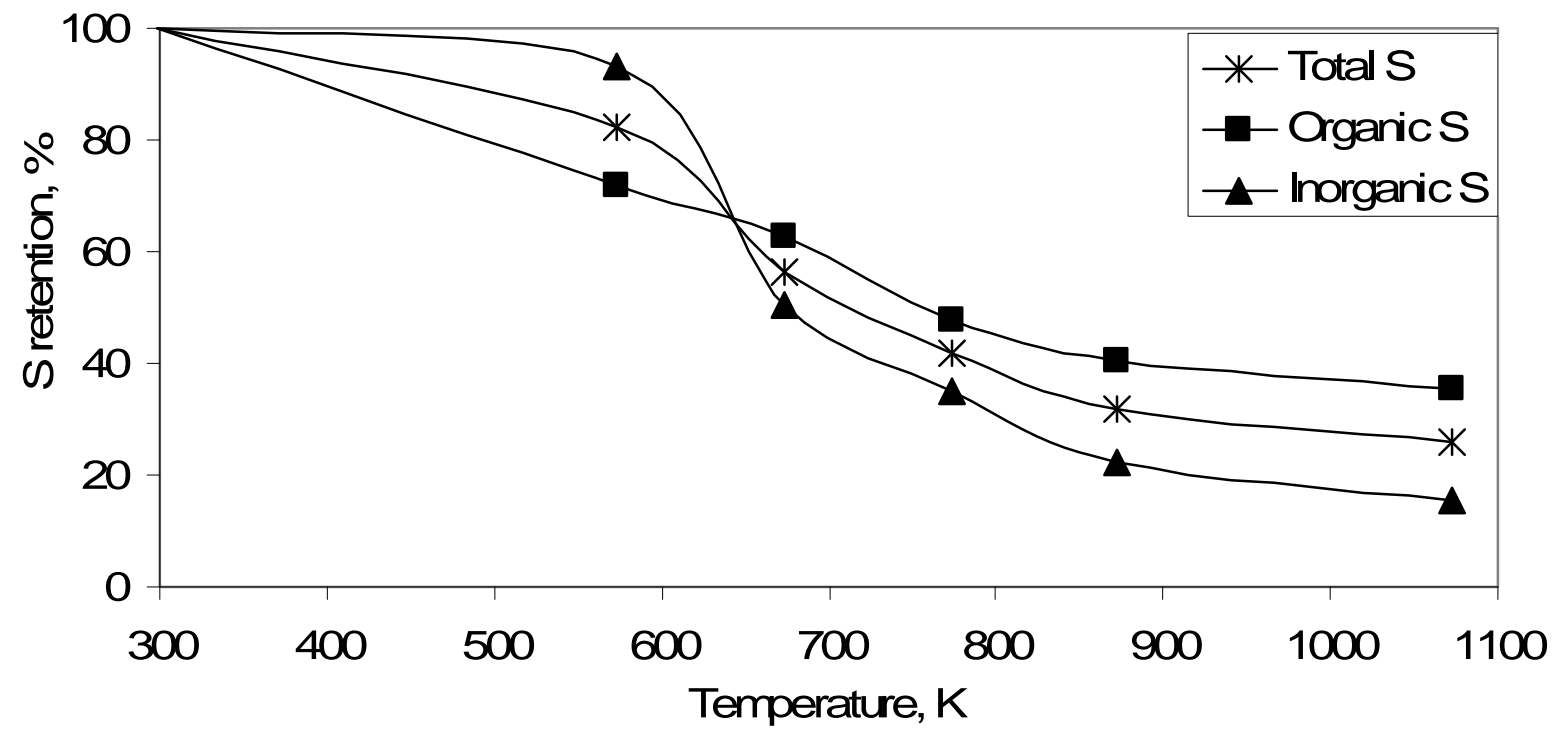

Figure 8.2 The retention of total sulphur, organic sulphur and inorganic sulphur in AWL2 chars during fixed bed pyrolysis

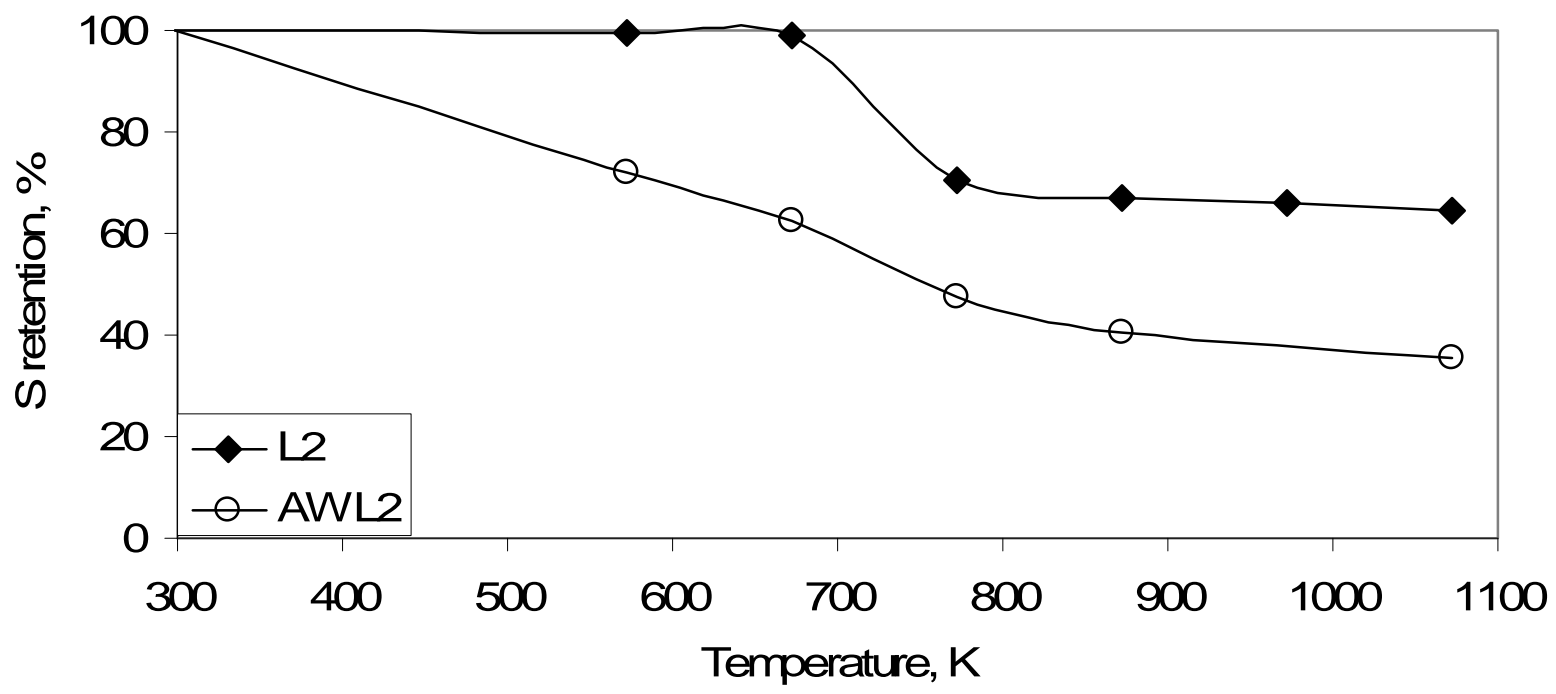

Figure 8.3 A comparison of organic sulphur in L2 and AW L2 chars during fixed bed pyrolysis 


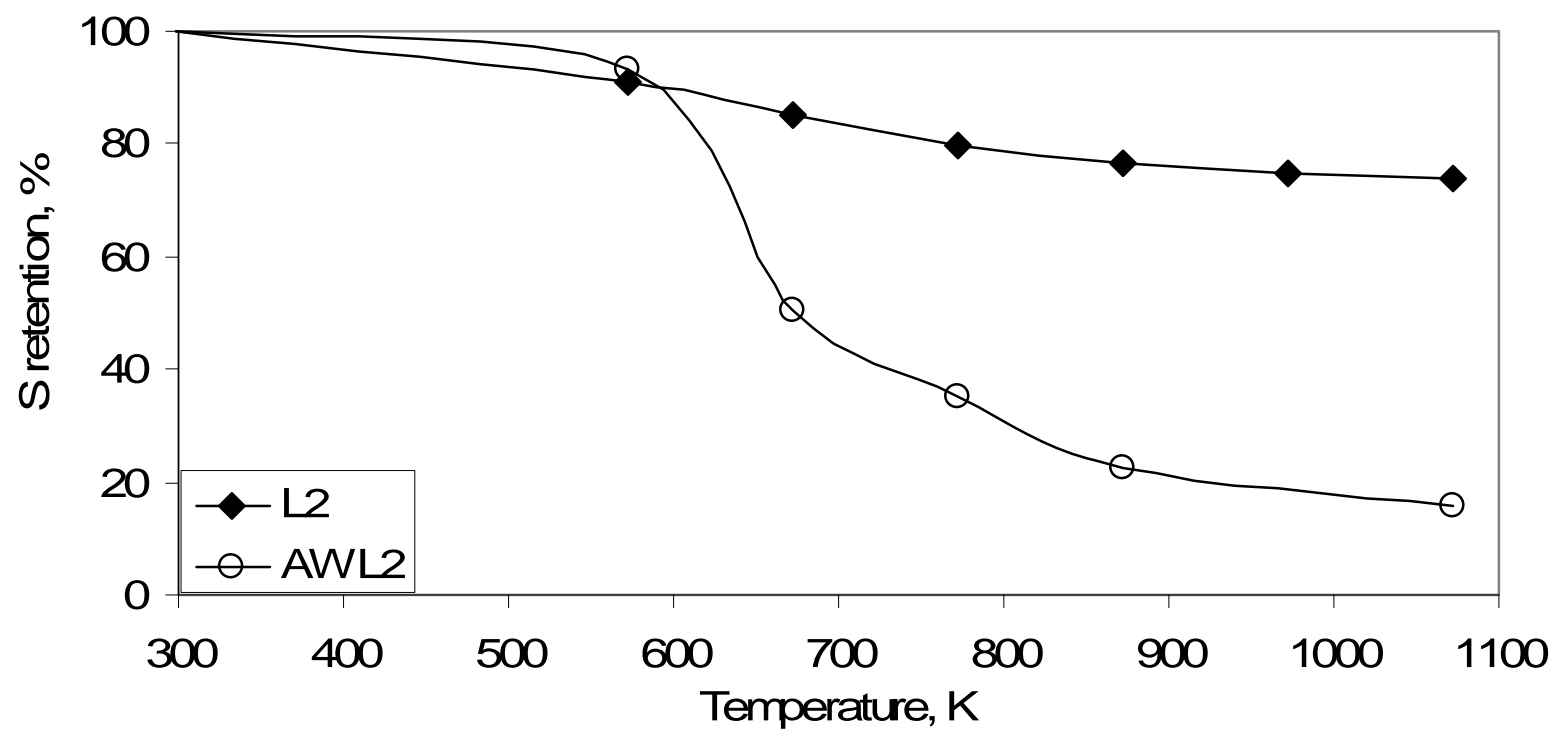

Figure 8.4 A comparison of inorganic sulphur retention in L2 and AW L2 chars during fixed bed pyrolysis

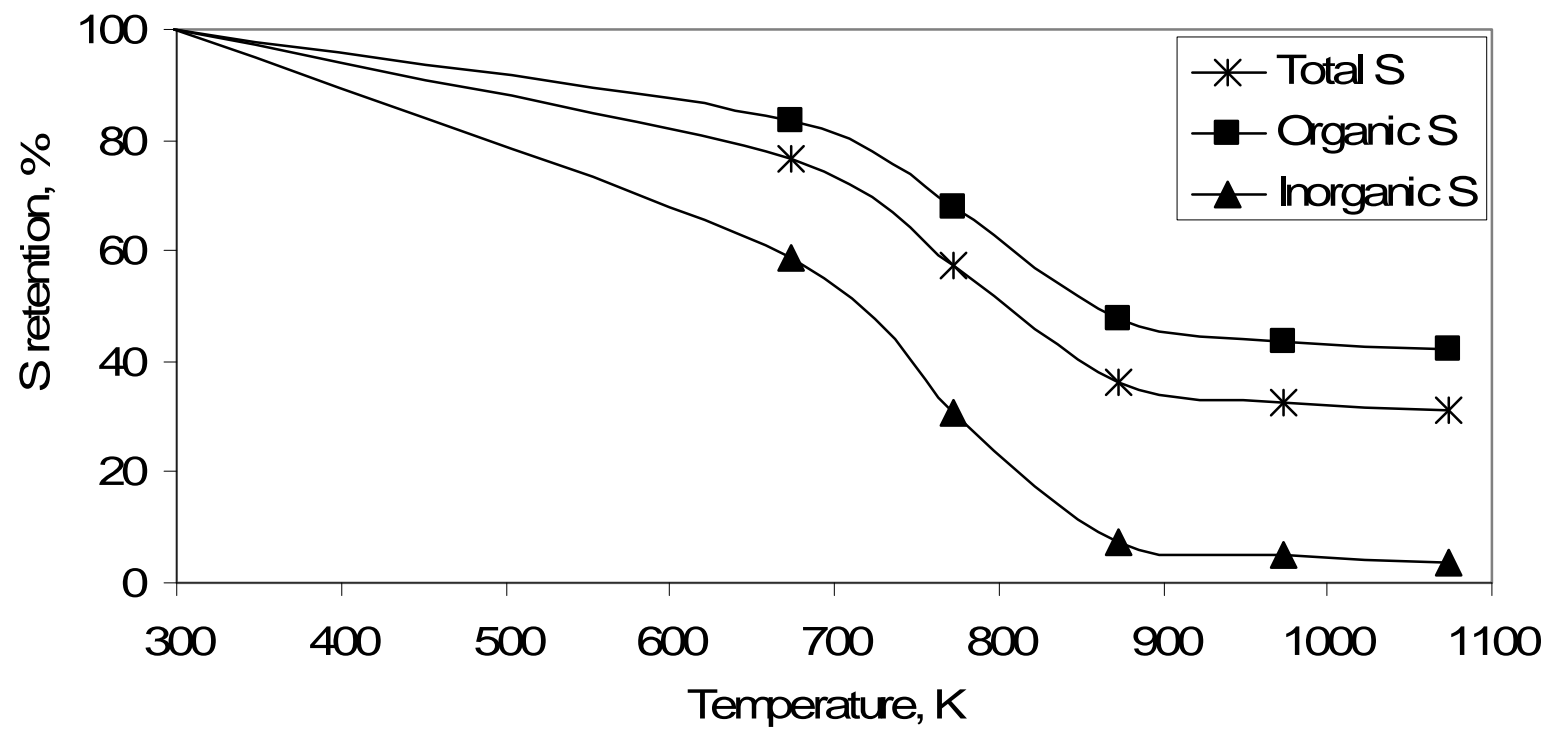

Figure 8.5 The retention of total sulphur, organic sulphur and inorganic sulphur in L3 chars during fixed bed pyrolysis 


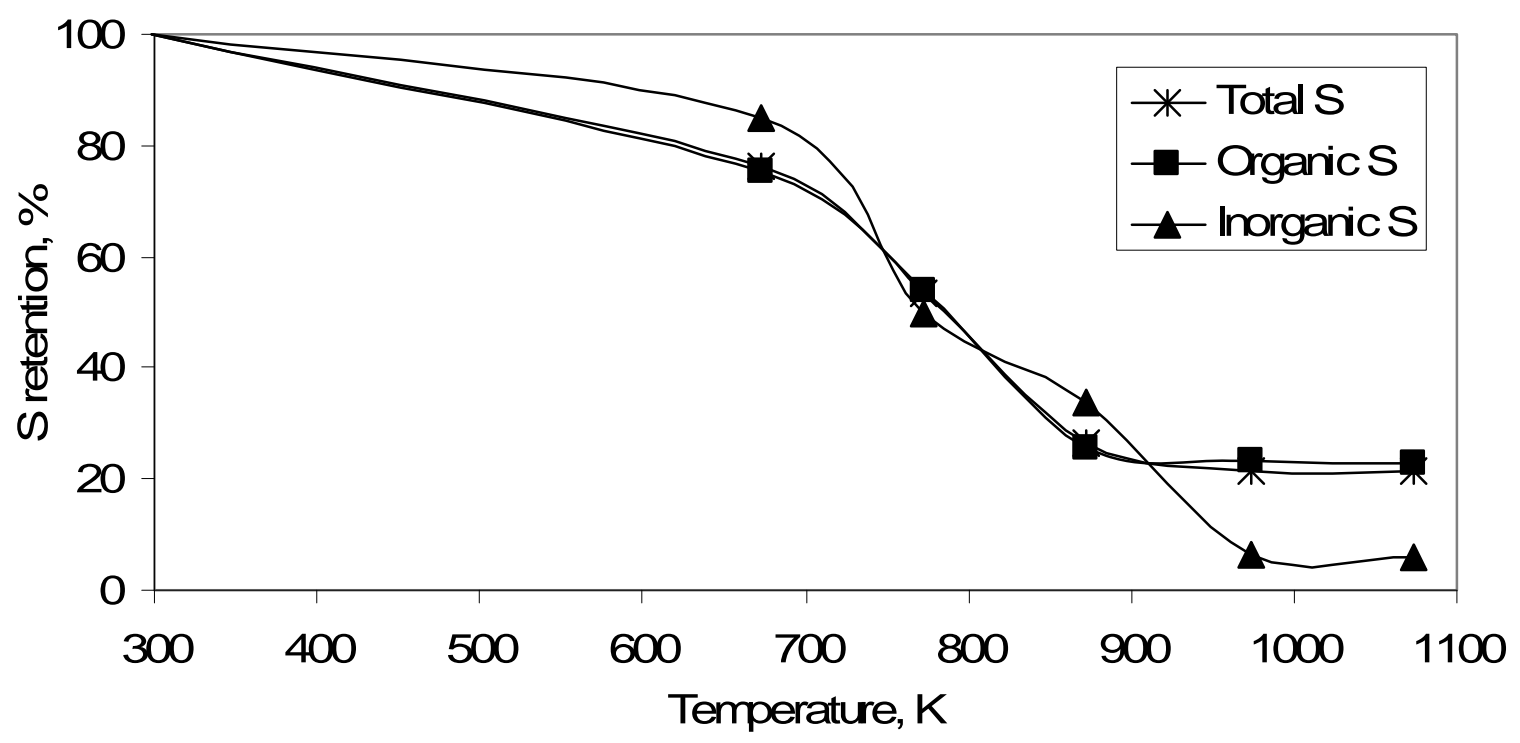

Figure 8.6 The retention of total sulphur, organic sulphur and inorganic sulphur in AWL3 chars during fixed bed pyrolysis

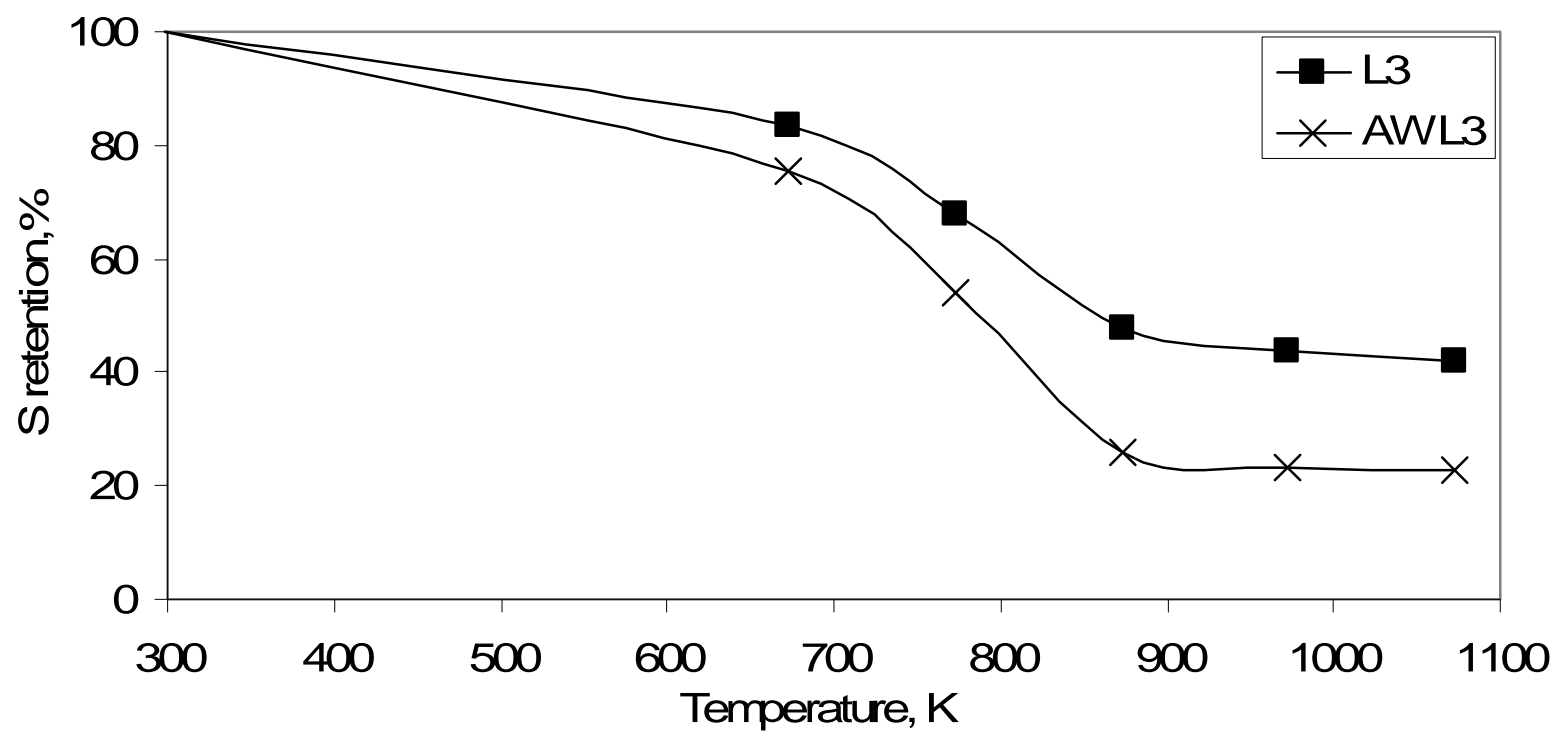

Figure 8.7 A comparison of organic sulphur retention in L3 and AW L3 chars during fixed bed pyrolysis 


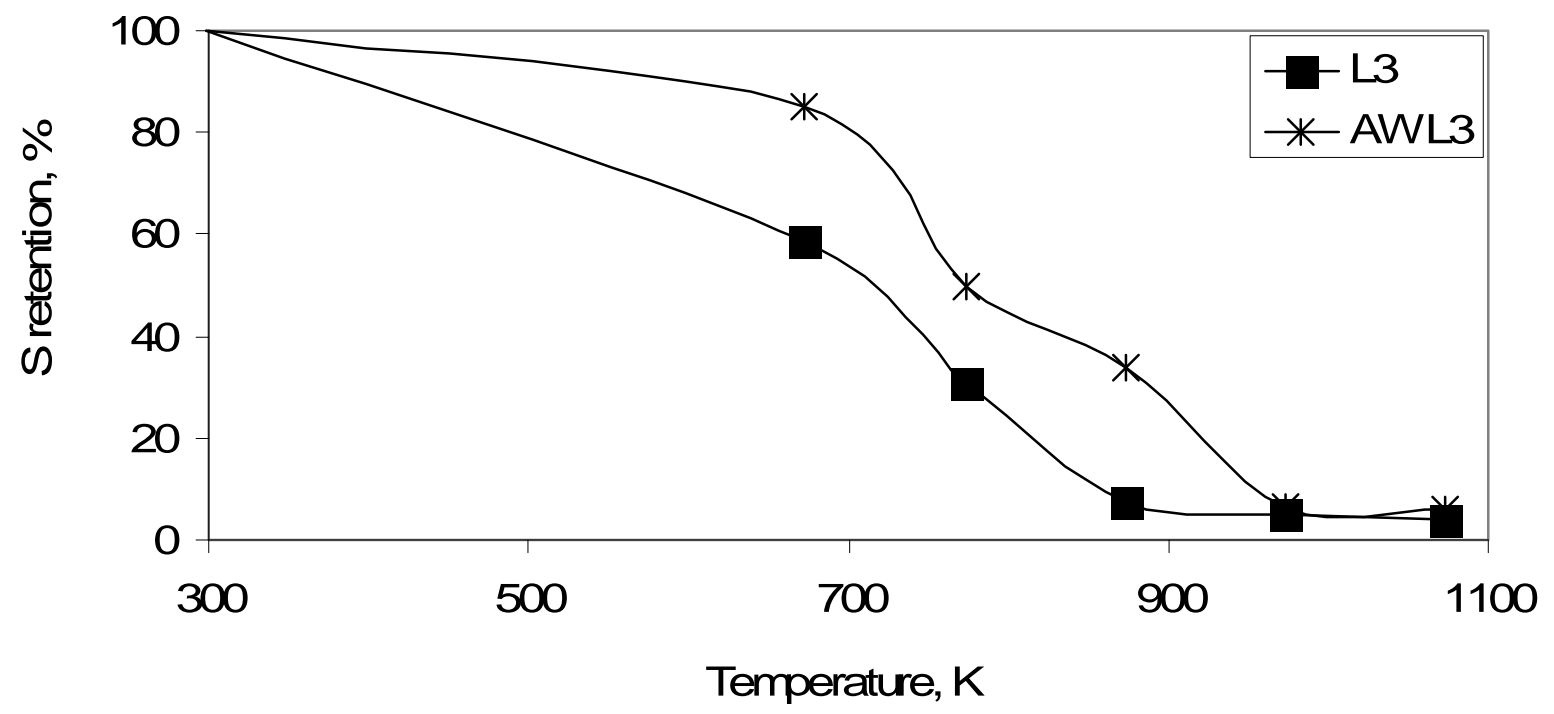

Figure 8.8 A comparison of inorganic sulphur retention in L3 and AW L3 chars during fixed bed pyrolysis

The retentions of total, organic and inorganic sulphur in L3 and AW L3 chars are displayed in Figures 8.5-8.8. The decomposition of organic and inorganic sulphurs in L3 char increases rapidly between $673-873 \mathrm{~K}$ and becomes slower above $873 \mathrm{~K}$ as observed from Figure 8.5. Most of the sulphur in L3 is dominated by organic sulphur, followed by sulphate and only a very small amount of pyrite. As discussed in Chapter 7, the organic sulphurs present in L3 are mostly labile organic sulphurs (thiols and sulphur-oxy compounds) which decompose at low temperatures. The inorganic sulphurs in the lignite are dominated by sulphates which decompose at about 573-773 K when surrounded by organic matter (ie, in a reducing environment) [2].

It can be seen from Figure 8.6 that the retention of the total and organic sulphur in AW L3 char is very similar, as most sulphur in AW L3 is in the form of organic sulphur (Table 4.3 Chapter 4). The retention of the total sulphur and organic sulphur for L3 and AW L3 decreases significantly between $673-873 \mathrm{~K}$ and becomes almost constant above $873 \mathrm{~K}$. From Figure 8.7 it can be seen that above $753 \mathrm{~K}$ the retention of organic sulphur in AWL3 char is lower than that in L3 char. This is due to the reactions of inherent 
Chapter 8

inorganic matter present in L3 with the organic sulphur-bearing compounds, similar to what happens in the case of L2 as discussed in the preceding paragraphs. SH- radicals produced from organic sulphur decomposition undergo complex reaction with inherent inorganic matter retaining the sulphur in the char. The inherent inorganic matter in L3 is also dominated by sodium with calcium only presents in a small quantity (Table 4.7 Chapter 4). As discussed previously, sodium and calcium begin to retain sulphur at around 773 and $873 \mathrm{~K}$. Above $873 \mathrm{~K}$ the organic sulphur retention in AW L3 char is about $20 \%$ lower than in L3. At $1073 \mathrm{~K}$ the retention of organic sulphur is about $42 \%$ for L3 char and about 23\% for AWL3 char.

As can be seen from Figure 8.8, the retention of inorganic sulphur in AW L3 char decreases more slowly than in L3 char between 673-873 K, and becomes more stable above $873 \mathrm{~K}$. This is due to the type of inorganic sulphurs present in the samples. The inorganic sulphur present in AW L3 is those not removed during the acid washing. The inorganic sulphur in the raw lignite is mainly sulphate sulphur. As discussed in Chapter 6 , the more volatile sulphates decompose at lower temperatures than the less volatile ones. In addition, the more volatile sulphates produce a greater amount of volatile sulphur than less volatile sulphates [2]. Since the raw lignite contains more less-stable sulphates, the decomposition of the inorganic sulphur is faster and, as a result, the inorganic sulphur retention decreases significantly. For AWL3, however, the retention of inorganic sulphur in the char is relatively greater.

\subsubsection{Effect of added inorganic matter}

To understand the role of inorganic matter in sulphur transformation during pyrolysis, sodium and kaolinite were added to AW L3. AW L3 doped with $\mathrm{Na}$ is called AW+Na, while AW L3 mixed with kaolinite is named AW+kaolin. Figure 8.9 shows SEM 
Chapter 8

images and EDS spectra of AW L3, AW+Na and AW+kaolin. It can be observed that $\mathrm{Na}$ and $\mathrm{Al}$ atoms are not detected by EDS spectra in AW L3 (Figure 8.9 (b)). In Figure 8.9 (d) it can be seen that sodium atom is detected by EDS. Al and Si atoms detected by EDS spectra as shown in Figure 8.9 (f) are confirmed that kaolinite is present in AW+kaolin sample.

Figure 8.10 shows a comparison of the total sulphur retention in L3 char, AW L3 char, $\mathrm{AW}+\mathrm{Na}$ and $\mathrm{AW}+$ kaolin chars, while the comparison of organic sulphur retentions for these samples is presented in Figure 8.11. It can be observed that profiles of total sulphur retention and organic sulphur retention are quite similar, because the organic sulphur is the dominant sulphur in L3 and AW L3 samples. Significant decreases in the total and organic sulphur retentions for all samples occur between 673-873 K. By adding $\mathrm{Na}$ to AW L3, the decomposition of organic sulphur is significantly reduced, the total and organic sulphur retentions in the $\mathrm{AW}+\mathrm{Na}$ char are the lowest among all samples in the temperature range studied. Kaolinite seems to enhance sulphur decomposition at lower temperatures between $673-773 \mathrm{~K}$, whereas above $873 \mathrm{~K}$, kaolinite retains the organic sulphur. It can be seen that there is an increase in the total and organic sulphur retentions in the AW+kaolin char above $873 \mathrm{~K}$. 


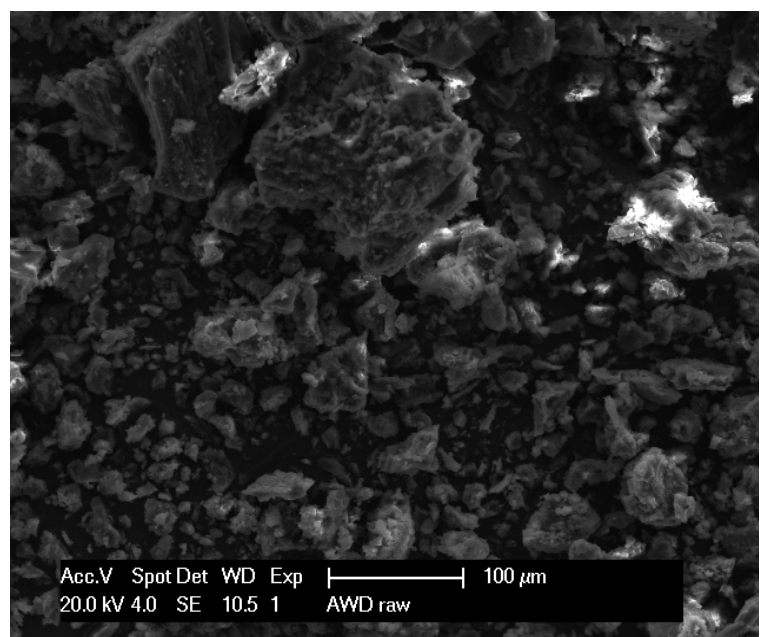

(a)

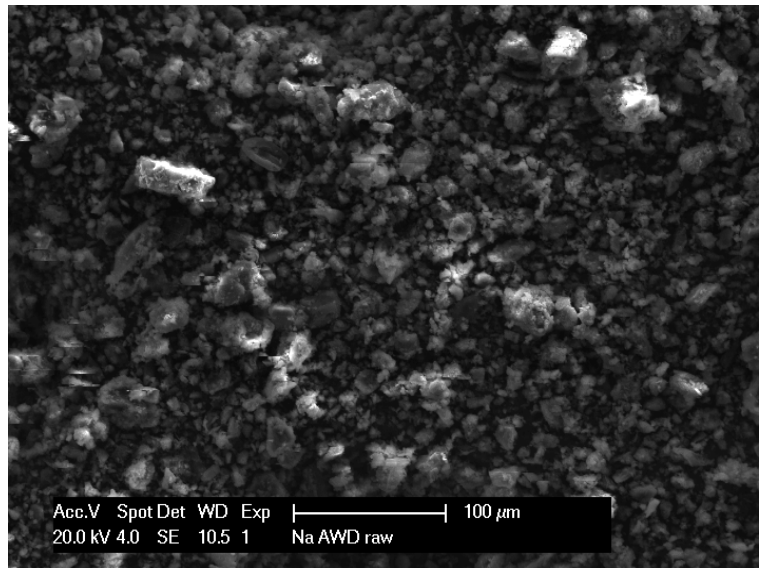

(c)

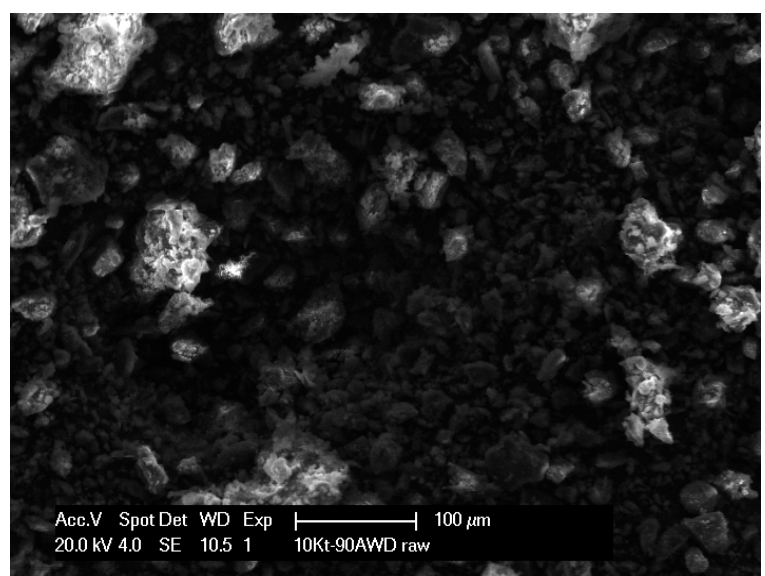

(e)

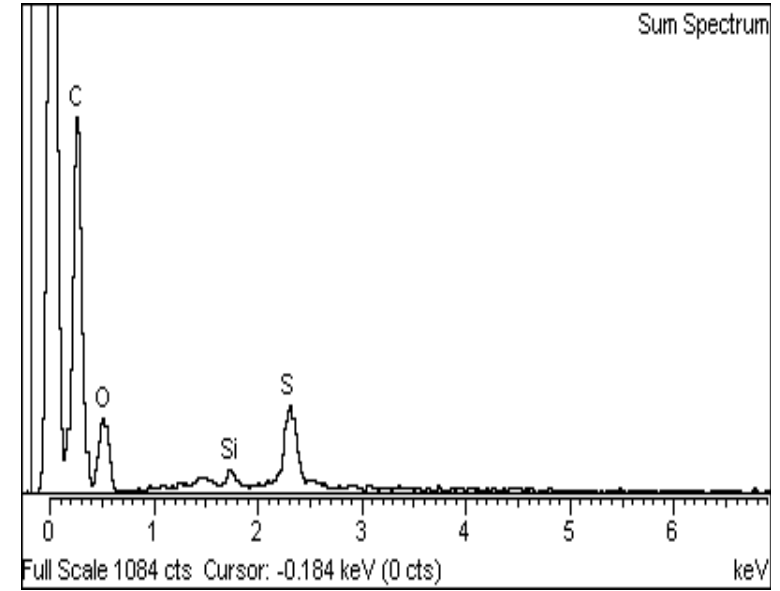

(b)

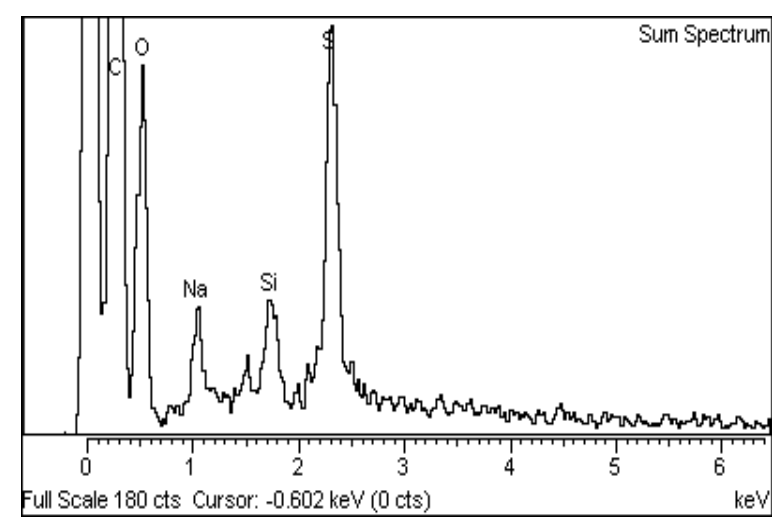

(d)

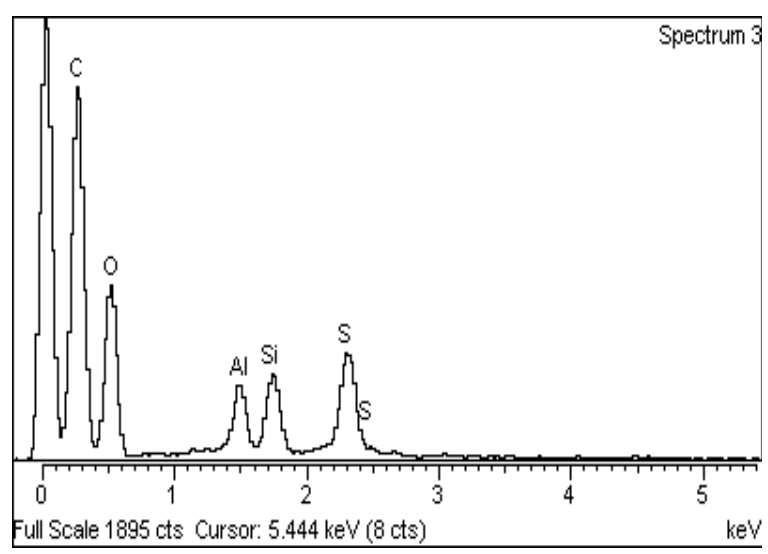

(f)

Figure 8.9 SEM images and EDS spectra of AW L3 (a-b), AW+Na (c-d) and AW+kaolin (e-f) 


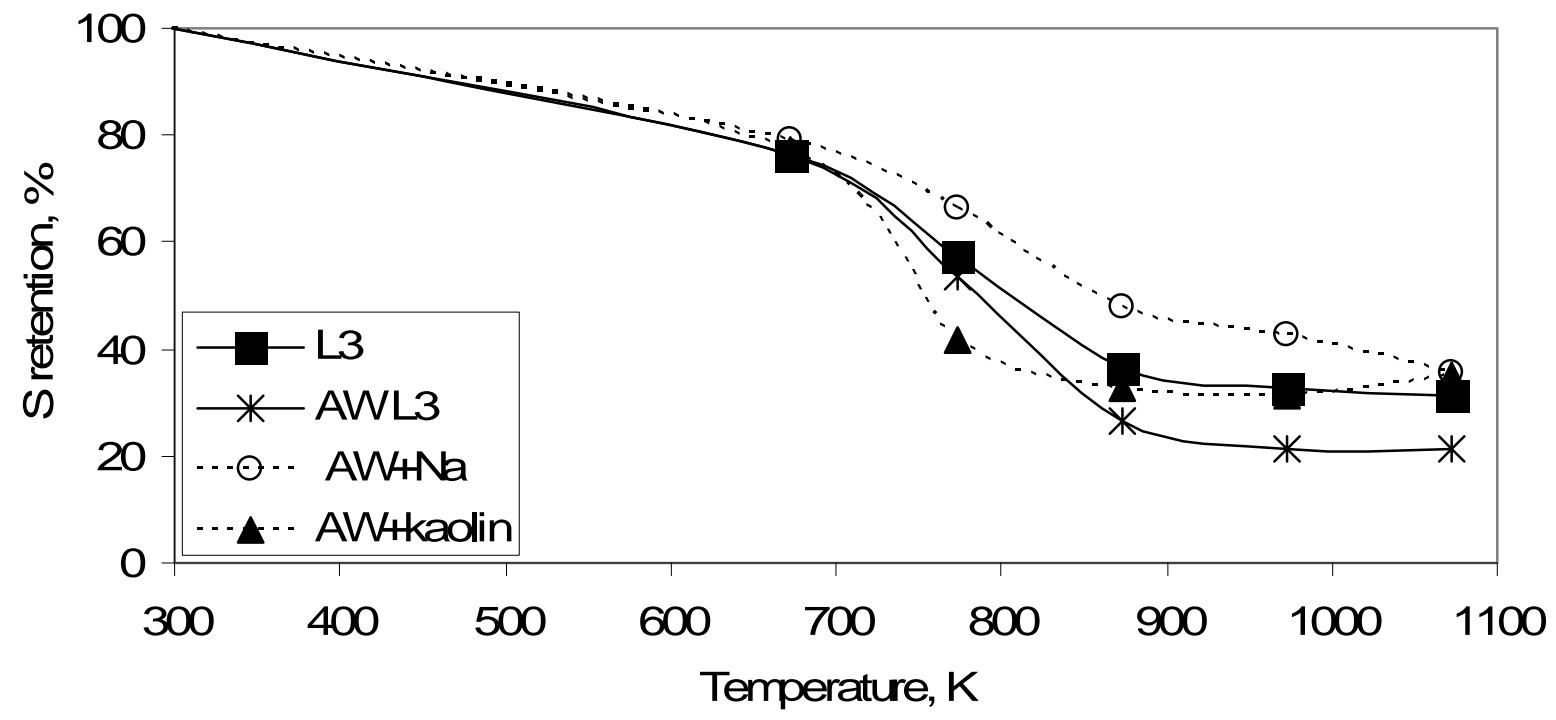

Figure 8.10 A comparison of total sulphur retention in L3, AW L3, AW+Na, $\mathrm{AW}+$ kaolin chars during fixed bed pyrolysis

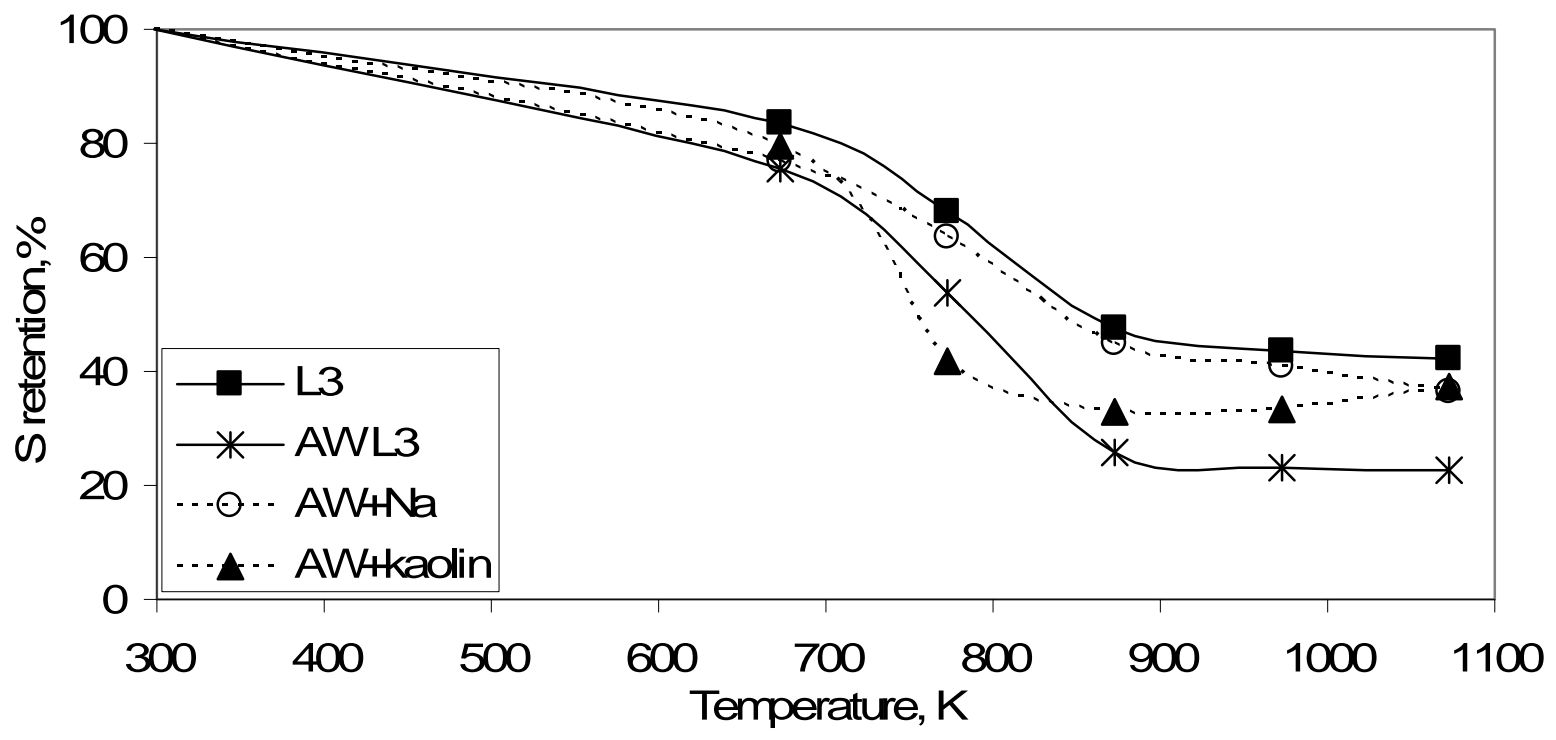

Figure 8.11 A comparison of organic sulphur retention in $L 3, A W L 3, A W+N a$, AW+kaolin chars during fixed bed pyrolysis 


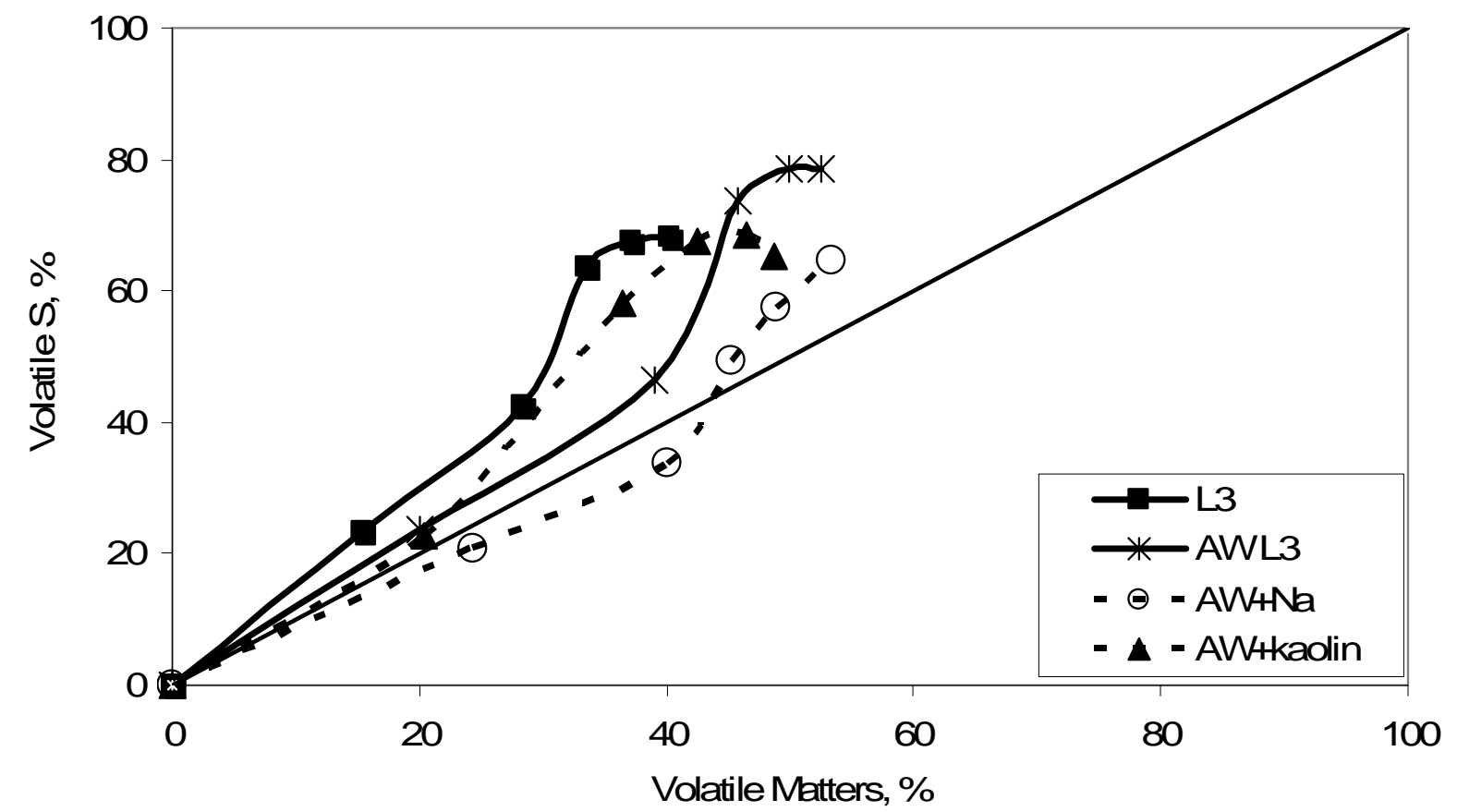

Figure 8.12 A comparison between volatile matter release and volatile sulphur release during pyrolysis of $\mathrm{L} 3, \mathrm{AW} \mathrm{L3}, \mathrm{AW}+\mathrm{Na}$ and $\mathrm{AW}+$ kaolin during pyrolysis in the fixed-bed reactor

To further study the effect of inorganic matter on sulphur transformation, a comparison between volatile matter and volatile sulphur released during pyrolysis of L3, AW L3, $\mathrm{AW}+\mathrm{Na}$ and $\mathrm{AW}+$ kaolin is made and presented in Figure 8.12. It is interesting to see that although the retention of sulphur in L3 char is higher than in AW L3 char, the evolution of volatile sulphur is faster than that of volatile matter for L3 than for AW L3. The release of volatile sulphur in the $\mathrm{AW}+\mathrm{Na}$ lignite char is the slowest among all samples. Between 673-773 K, the evolution of volatile sulphur in the $\mathrm{AW}+\mathrm{Na}$ lignite is much lower than the evolution of volatile matter and only becomes significant above $873 \mathrm{~K}$. This is explained by the fact that $\mathrm{Na}$ is more reactive towards $\mathrm{SH}^{*}$ radicals to retain sulphur in the char but, at higher temperatures, $\mathrm{Na}$ becomes more volatile itself and therefore, its ability to retain sulphur in the char is reduced above $873 \mathrm{~K}$. The pyrolysis of the AW+kaolin initially shows higher volatile sulphur evolution than AW L3 but, at higher temperatures, the evolution of sulphur becomes slower compared to 
Chapter 8

AW L3. This is because kaolinite has a lower reactivity towards sulphur at low temperatures but as temperature increases, kaolinite can react with sulphur to form more stable sulphates, thus, its ability to retain sulphur is greater at high temperatures

\subsection{Summary}

The retention of organic and inorganic sulphur in L3 is higher than in AW L3. This is due to the interaction between inherent inorganic matter in L3 with organic sulphur, retaining the organic sulphur in the char. Inorganic matter also reacts with the pyrite decomposition products resulting another inorganic sulphur retained in the char.

The retention of organic sulphur L3 is higher than in AW L3 due to the inherent inorganic matter present in the raw lignite which readily reacts with the sulphur to retain sulphur in the char. The decomposition of inorganic sulphur in L3, however, is not affected by the inherent inorganic matter; it depends on the type of sulphates present in the sample.

Adding kaolinite and sodium to AW L3 affects the sulphur retention during pyrolysis. Sodium has a retention effect on the organic sulphur between $673-773 \mathrm{~K}$, while kaolinite has a better ability to retain sulphur above $873 \mathrm{~K}$. 


\section{CHAPTER 9}

\section{Evaluation and Practical Implications}

\subsection{Introduction}

In this chapter the present work on sulphur transformation as detailed in the preceding chapters is evaluated with the reference to the objectives set out in Chapter 2 and benchmarked against the literature. In evaluating the work, a comparison between the current studies with previous studies is also presented wherever appropriate. Based on the evaluation, the conclusions of this thesis work and recommendations for future studies are drawn.

\subsection{Pyrite transformation during lignite pyrolysis}

The study of pyrite transformation during lignite pyrolysis presented in Chapter 5 has shown that pyrite which is surrounded by organic matrix of the lignite decomposes at a lower temperature than pyrite mineral alone. By comparing the $\mathrm{S} / \mathrm{Fe}$ ratio in the chars recovered from the pyrolysis of pyrite mineral in a TGA under nitrogen with a particle size $<150 \mu \mathrm{m}$ at a heating rate of 10 and $50 \mathrm{~K} \cdot \mathrm{min}^{-1}$, it was confirmed that pyrite decomposes to pyrrothite at ca $720 \mathrm{~K}$ and then to troilite at $1200 \mathrm{~K}$. Some of the troilite decomposes to elemental iron and gaseous elemental sulphur above $1200 \mathrm{~K}$. While during pyrolysis of lignite with high pyrite content as well as samples of the lignite and pyrite blends, pyrite was shown to decompose at about $100 \mathrm{~K}$ lower than the decomposition temperature of pyrite mineral. 
Table 9.1 Comparison of experimental techniques and conditions for thermal decomposition of pyrite between the current study and that of Chen et al [7].

\begin{tabular}{|c|c|c|}
\hline & Current study & Chen et al [7] \\
\hline Particle size, $\mu \mathrm{m}$ & $<150$ & $<75$ \\
\hline Sample weight, mg & $\sim 12$ & $\sim 50$ \\
\hline Gas atmosphere & nitrogen & nitrogen and hidrogen \\
\hline Gas flow rate, $\mathrm{ml}^{\mathrm{min}}{ }^{-1}$ & 100 & 180 \\
\hline Heating rate, K.min ${ }^{-1}$ & 10 and 50 & 5 \\
\hline Pressure & atmospheric & $0.1-5 \mathrm{MPa}$ \\
\hline Reactor & ceramic crucible in a TGA & quartz crucible \\
\hline
\end{tabular}

A comparison of pyrite thermal decomposition studied by Chen et al [7] and the current work is detailed in Table 9.1. The pyrite decomposition in Chen et al's [7] study can be described with the following steps:

$\mathrm{FeS}_{2} \rightarrow \mathrm{FeS}_{1.2} \rightarrow \mathrm{FeS}(0.1 \mathrm{MPa}$ nitrogen; temperature $<1223 \mathrm{~K}$

$\mathrm{FeS} 2 \rightarrow \mathrm{FeS}_{1.34}(3 \mathrm{MPa}$ nitrogen; temperature $<1073 \mathrm{~K})$

$\mathrm{FeS}_{2} \rightarrow \mathrm{FeS} \rightarrow \mathrm{Fe}$ (hydrogen)

From the pyrite decomposition steps (R8.1-R8.3), it can be seen that elemental iron was formed only under hydrogen atmosphere. In the current study, however, it was shown that elemental iron was formed during thermal decomposition of pyrite in a nitrogen atmosphere. The pyrite transformation under nitrogen can be described with the following paths:

$\mathrm{FeS}_{2} \rightarrow \mathrm{FeS}_{\mathrm{x}} \rightarrow \mathrm{FeS} \rightarrow \mathrm{Fe}$ 
Chapter 9

The pyrite decomposition steps during thermal decomposition in nitrogen presented above (R8.4) agrees with the pyrite decomposition steps under inert atmosphere proposed by $\mathrm{Hu}$ et al [51].

It should be noted that thermal decomposition of pyrite in the present study were only examined in the nitrogen atmosphere, slow heating rate $\left(10\right.$ and $\left.50 \mathrm{~K} \cdot \mathrm{min}^{-1}\right)$ and with particle size $<150 \mu \mathrm{m}$ as can be seen from Table 8.1 . For future study it should be extended to consider different gas atmospheres, fast heating rate and various ranges of particle sizes.

During coal pyrolysis, pyrite interacts with organic matrix of the coal to evolve hydrogen sulphide according to the following reaction [56]:

$\mathrm{FeS}_{2}+(\mathrm{x}-2) \mathrm{H}_{2} \rightarrow \mathrm{FeS}_{\mathrm{x}}+(2-\mathrm{x}) \mathrm{H}_{2} \mathrm{~S}$

In Chapter 5 it was shown that in lignite blends with pyrite samples, $\mathrm{H}_{2} \mathrm{~S}$ is found to be the dominant sulphur gas evolved during pyrolysis. However, during pyrolysis of lignite with high inherent pyrite content in a TGA-MS in the present study $\mathrm{H}_{2} \mathrm{~S}$ was not detected. The high oxygenated functional groups present in the lignite affect the gaseous sulphur evolution. Inherent pyrite interacts with lignite organic matrix which contains high oxygenated structures to produce $\mathrm{SO}_{2}$ rather than $\mathrm{H}_{2} \mathrm{~S}$. Inherent pyrite is deeply embedded in the coal matrix, whereas added pyrite is considered extraneous. Therefore, the added pyrite behaves somewhat differently in the lignite organic matrix.

The lignite used in the experimentation also contains very high inorganic matter. The presence of inorganic matter in the lignite helps to retain sulphur, both inorganic and organic sulphur, in the solid phase. Mineral matter has been found to fix $\mathrm{H}_{2} \mathrm{~S}$ evolved from sulphur decomposition to form higher sulphur content in the chars $[2,101]$. 
Chapter 9

In this study the forms of sulphur left in the char were only presented as total, inorganic and organic sulphur. Several complimentary analyses, such as FTIR, SEM-EDS and XRD helped interpret the pyrite transformation. However, those analyses were not able to quantify the forms of the sulphur remained in the chars.

Kinetic study of pyrite, lignites, and the blended pyrite-lignite samples during pyrolysis were performed. The kinetic parameters are in accordance with previous studies [3, 57, 111]. It is suggested that the kinetic study is performed not only on the kinetics of the whole lignite pyrolysis, but also on the pyrite itself during lignite pyrolysis. To be able to study the kinetics of pyrite during lignite pyrolysis, it will require data of the sulphur forms and contents in the chars as well as gaseous sulphur evolved from the pyrite decomposition.

\subsection{Sulphate transformation during lignite pyrolysis}

It was discussed in Chapter 6 that when surrounded by organic matrix of lignite, sulphate decomposes at lower temperature than the decomposition of its pure sulphate as also shown by previous studies $[9,64,112]$. During thermal decomposition in a TGA using two different atmospheres, namely, nitrogen and a mixture of $50 \%$ nitrogen + $50 \%$ carbon dioxide, calcium sulphate decomposes between 1400-1700 K and iron (II) sulphate decomposes between 813-953 K. Whereas, $\mathrm{CaSO}_{4}$ in $\mathrm{CaSO}_{4}$ impregnated in low-sulphur lignite $\left(\mathrm{CaSO}_{4}+\mathrm{L} 1\right)$ starts to decompose at $623 \mathrm{~K}$ and $\mathrm{FeSO}_{4}$ in $\mathrm{FeSO}_{4}$ impregnated in low-sulphur lignite $\left(\mathrm{FeSO}_{4}+\mathrm{L} 1\right)$ begin to decompose at $500 \mathrm{~K}$.

A comparison between the current research and previous research by Yan et al [9] was made and shown in Table 9.2. It can be seen that $\mathrm{FeSO}_{4}$ in $\mathrm{FeSO}_{4}+\mathrm{L} 1$ decomposed to evolve $\mathrm{SO}_{2}$ from $500 \mathrm{~K}$ to $1000 \mathrm{~K}$. During temperature-programmed decomposition (TPD) of $\mathrm{Fe}_{2}\left(\mathrm{SO}_{4}\right)_{3}$ impregnated in a char by Yan et al [9] it is shown that $\mathrm{SO}_{2}$ is the 
Chapter 9

only sulphur gas evolved which evolves from 473-800 K. This confirms our conclusion that the sulphates in L4 is dominated by iron sulphates which decomposed to evolve $\mathrm{SO}_{2}$ between 473-917 K.

Table 9.2 A comparison of sulphate decomposition in the current study and that of Yan et al [9]

\begin{tabular}{|c|c|c|c|}
\hline & $\mathrm{FeSO}_{4}+\mathrm{L} 1$ & L4 & $\mathrm{Fe}_{2}\left(\mathrm{SO}_{4}\right)_{3}$ in a char[9] \\
\hline Method & TGA-MS & TGA-MS & TPD-MS \\
\hline Gas environment & Argon & Argon & Helium \\
\hline Heating rate, K.min ${ }^{-1}$ & 10 & 10 & 5 \\
\hline Sulphur gas evolved & $\mathrm{SO}_{2}$ & $\mathrm{SO}_{2}$ & $\mathrm{SO}_{2}$ \\
\hline $\begin{array}{l}\text { Temperature range of } \\
\text { the } \mathrm{SO}_{2} \text { evolution, } \mathrm{K}\end{array}$ & $500-1000$ & $473-800$ & $473-917$ \\
\hline
\end{tabular}

$\mathrm{CaSO}_{4}$ has a lower volatility than $\mathrm{FeSO}_{4}[61,112]$. In the present study it was shown that most of $\mathrm{FeSO}_{4}$ in $\mathrm{FeSO}_{4}+\mathrm{L} 1$ are decomposed during lignite pyrolysis, whereas only small portion of $\mathrm{CaSO}_{4}$ in $\mathrm{CaSO}_{4}+\mathrm{L} 1$ impregnated in lignite are decomposed.

It is discussed in Chapter 6 that $\mathrm{CaSO}_{4}$ is a stable compound; it hardly decomposes at high temperature up to $1400 \mathrm{~K}$. Therefore, the presence of $\mathrm{CaSO}_{4}$ in coal will not give significant effect in producing sulphurous related pollution gases.

In the present study the effect of inorganic matter on the transformation of sulphate during lignite pyrolysis has not yet been covered. It is suggested to study both effects of inherent and added inorganic on the sulphate transformation during pyrolysis. It is also further suggested to study other sulphates which may also present in lignite to give thorough understanding on the sulphate transformation during lignite pyrolysis. 


\subsection{Organic sulphur transformation during lignite pyrolysis}

It is discussed in Chapter 7 that naphthalene thiol in the impregnated lignite $\mathrm{NT}+\mathrm{L} 1$ and benzyl sulphoxide in the impregnated lignite $\mathrm{BS}+\mathrm{L} 1$ decompose at a temperature lower than $673 \mathrm{~K}$. The inherent organic sulphur retention in L3 is higher than AW L3 at the corresponding pyrolysis temperatures due to the interaction between organic sulphur with inherent inorganic matter in L3.

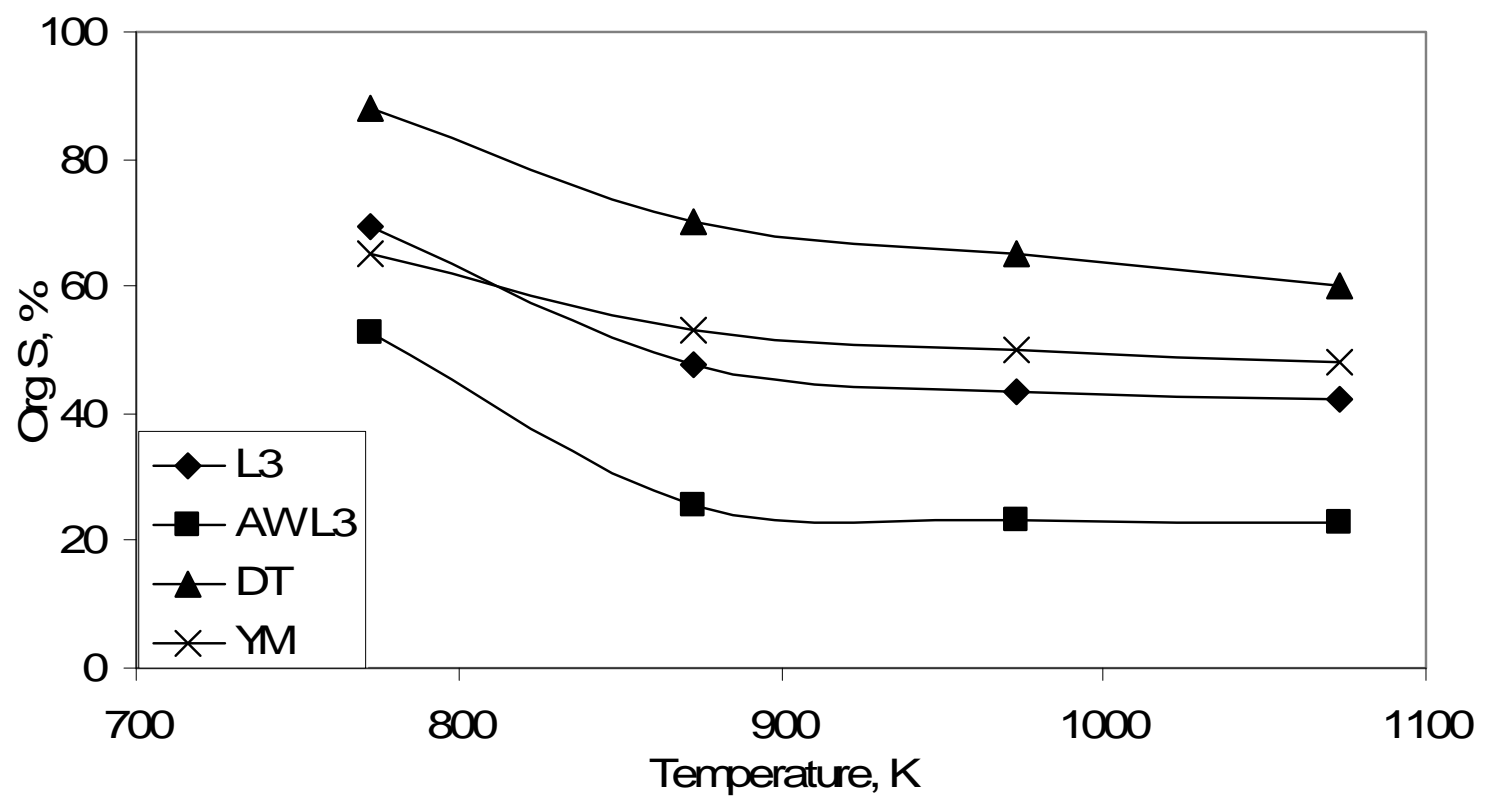

Figure 9.1 Comparison of retentions of organic sulphur during pyrolysis of L3 and AW L3 in a fixed bed reactor with pyrolysis of Datong (DT) and Yima (YM) in a fluidized bed reactor by Qi et al [127]

A comparison of organic sulphur retentions between the current study and literature studies on Datong (DT) and Yima (YM) coal by Qi et al [127] was made as shown in Table 9.3. It can be seen that the sulphur forms in L3 is dominated by organic sulphur, whereas pyritic sulphur is dominant in DT and YM. As discussed in Chapter 7, the organic sulphur in L3 is from the labile organic sulphur, while YM coal has more labile organic sulphur than DT [127]. The current study was carried out in a fast heating rate 
Chapter 9

fixed bed pyrolysis, whereas Qi et al [127] study was carried out in a fast heating rate fluidised bed pyrolysis.

Table 9.3 A comparison of sulphur forms analysis and experimental data of current study and Qi et al [127] study

\begin{tabular}{|l|l|l|l|}
\hline & L3 & Datong [127] & Yima [127] \\
\hline Sulphur forms, \% (db) & & & \\
$\mathrm{S}_{\text {total }}$ & 2.18 & 1.04 & 1.95 \\
$\mathrm{~S}_{\text {sulphate }}$ & 0.56 & 0.06 & 0.08 \\
$\mathrm{~S}_{\text {pyritic }}$ & 0.06 & 0.62 & 1.42 \\
$\mathrm{~S}_{\text {organic }}$ & 1.56 & 0.36 & 0.45 \\
\hline Reactor & Fixed bed & Fluidised bed & Fluidised bed \\
\hline Heating rate & Fast & Fast & Fast \\
\hline Temperature ranges, K & $673-1073$ & $773-1173$ & $773-1173$ \\
\hline Residence time, min & 30 & 30 & 30 \\
\hline
\end{tabular}

Figure 9.1 shows the results of organic sulphur retentions in the chars derived from the fixed bed pyrolysis experiments of AW L3 and L3 samples compared with the organic sulphur retentions in the chars DT and YM. It can be seen that the retentions of organic sulphur in the chars of L3, AW L3 and YM decrease significantly until $873 \mathrm{~K}$, then level off. Organic sulphur in DT behaves differently, the retentions of organic sulphur in DT decrease significantly until $873 \mathrm{~K}$, then above $873 \mathrm{~K}$ there is still a little decrease in the retention of organic sulphur. The type of organic sulphur in the coal affects its behaviour. The organic sulphur compounds in L3, AW L3 and YM are mostly from less stabile organic sulphur, eg. aliphatic sulphur, which decomposes at lower temperature $(\sim 673 \mathrm{~K})$, whereas more thiophenes present in DT; thiophenes decompose at high temperature (ca $1023 \mathrm{~K}$ ) [127]. This is the reason for the lower retentions of organic sulphur in L3, AW L3 and YM compare to in DT. 
Chapter 9

The retention of organic sulphur in the present study was only performed at one residence time, namely, 30 minutes, and one atmosphere, namely, nitrogen. It is worthy to study the effect of residence times as well as atmosphere gases on the sulphur transformation during lignite pyrolysis.

L3 has higher retentions of organic sulphur in the chars at corresponding pyrolysis temperatures than in AW L3. Qi et al [127] also noted that the retentions of organic sulphur in the chars of raw coals are higher than in the chars of their demineralised samples; inherent inorganic matter is mainly contributed to the higher retention of organic sulphur in the chars of raw coal than in its demineralised sample. Qi et al [127] concluded that the interaction of pyrite with organic matrix of coal also plays an important role in organic sulphur retention in the char.

Added inorganic matter, namely, sodium and kaolinite also affects the transformation of organic sulphur during lignite pyrolysis. It is worth to study the effect of other inorganic matter on the sulphur transformation during lignite pyrolysis.

During pyrolysis, organic sulphur in the coal decomposes to form -SH radicals [127]. Some of the $-\mathrm{SH}$ radicals incorporate in the char, while others undergo complex reactions with organic matrix of the coal to produce gaseous sulphur, mainly of $\mathrm{H}_{2} \mathrm{~S}$ $[127,130]$. However, during pyrolysis of L3 and AW L3 which contain high organic sulphur, there is no $\mathrm{H}_{2} \mathrm{~S}$ detected during pyrolysis, $\mathrm{SO}_{2}$ is the only gas sulphur evolves during pyrolysis of L3 and AW L3. During pyrolysis of L3 and AW L3, some of the $\mathrm{SH}$ radicals produce from the decomposition of organic sulphur react with organic matrix of the lignite which have high oxygenated structures to produce $\mathrm{SO}_{2}$.

Due to limitation of the analytical instrumentations, organic sulphur functionalities present in the lignites and their chars could not be identified. Knowing the 
Chapter 9

functionalities of organic sulphur compounds in the coal will give better understanding of the behaviour of organic sulphur during lignite pyrolysis.

TGA-MS has given data of evolved gases during lignite pyrolysis. However, some of the compounds have similar $\mathrm{m} / \mathrm{z}$ values, such as nitrogen $(\mathrm{m} / \mathrm{z} 28)$ has the same $\mathrm{m} / \mathrm{z}$ value with CO. Another drawback of MS is that the gas could have more than one $\mathrm{m} / \mathrm{z}$ signal. Therefore, it will give more precise detections of the gases evolution if apart from MS, other analytical instruments, such as gas chromatography (GC) or FTIR is also used. Thus, it will help to reveal the mechanism of each type of organic sulphur during lignite pyrolysis.

\subsection{A proposed mechanisms of sulphur transformation during}

\section{lignite pyrolysis}

During lignite pyrolysis, there are complex interactions between pyrite and organic matrix of the lignite which is highly oxygenated to lead the evolution of $\mathrm{SO}_{2}$ as the gaseous sulphur. The interaction between pyrite and inorganic matter of the lignite help retaining some of the sulphur from pyrite decomposition in the char. Pyrite may transform to sulphate sulphur and organic sulphur during pyrolysis.

Sulphur dioxide is the gaseous sulphur evolved from the sulphate decomposition during lignite pyrolysis. The interaction between sulphate sulphur and organic matrix as well as inorganic matter of the lignite results in the formation of organic sulphur. However, sulphate decomposition during lignite pyrolysis will never produce pyritic sulphur.

During pyrolysis, organic sulphur decomposes to form $-\mathrm{SH}$ radicals. The radicals then will undergo complex interactions with organic matrix of the lignite to produce various sulphur compounds such as $\mathrm{H}_{2} \mathrm{~S}, \mathrm{COS}, \mathrm{CS}_{2}, \mathrm{SO}_{2}$ and elemental sulphur and also other 
Chapter 9

sulphur forms retained in the solid phase [9]. The high oxygenated organic matrix of this lignite lead to the evolution of only $\mathrm{SO}_{2}$ from the decomposition of organic sulphur during pyrolysis. The interaction of organic sulphur with inorganic matter help the retention of the sulphur formed from the organic sulphur decomposition in the char, in the form of other organic sulphur compounds and sulphate sulphur.

From the preceding paragraphs the mechanisms of the sulphur transformation during lignite pyrolysis have been proposed as articulated in Figure 9.2.

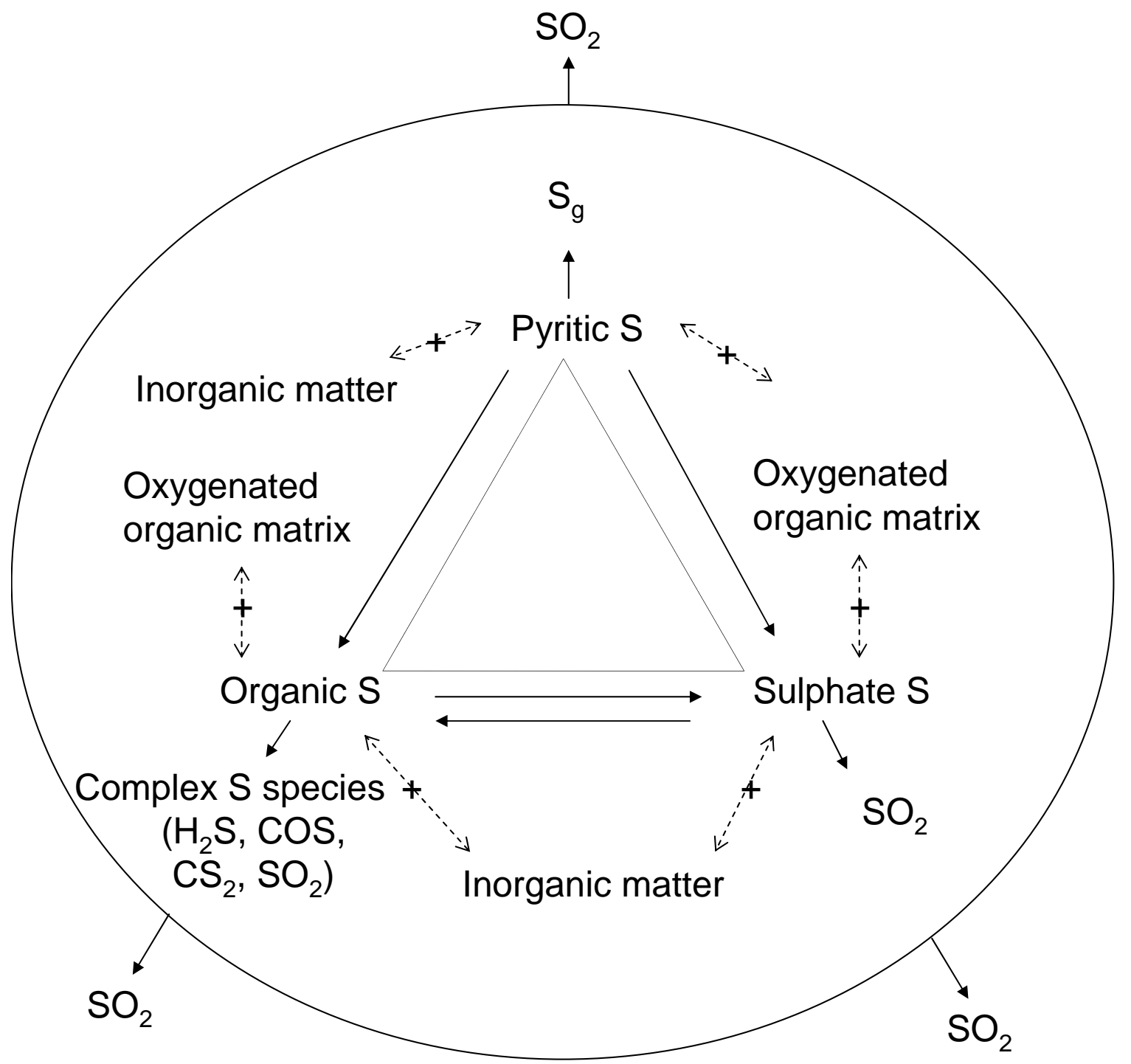

Figure 9.2 A schematic of the mechanisms of sulphur transformation during lignite pyrolysis 


\subsection{Practical implications}

The present study has improved fundamental understandings of the sulphur transformation during pyrolysis of lignites which contain high oxygenated functional groups and extremely high inorganic matter, especially sodium and chlorine. Thus, the environmental and processing issues regarding the utilisations of lignite with high oxygenated groups as well as inorganic matter could be addressed.

The interaction between sulphur and high-oxygenated organic matrix of the lignite during pyrolysis leads to the evolution of sulphur dioxide instead of hydrogen sulphide. It was also confirmed that $\mathrm{CaSO}_{4}$ presents in the lignite is a stable compound until high pyrolysis temperature. Inherent inorganic matter in the lignite interacts with sulphur during pyrolysis to help retaining sulphur in the solid phase. The findings have various practical implications regarding processing and developing appropriate sulphur capture strategies during pyrolysis and other thermochemical conversion of lignite or other coals with high oxygenated functional groups and high inorganic matter.

During combustion, the char is oxidized to form ash and release gases. The proportion and forms of sulphur present in the ash can be predicted. For example, $\mathrm{CaSO}_{4}$ which is thermally stable in high temperature is expected to present in the ash. During gasification, the char reacts with gasifying agent under reducing environment. The interaction of sulphur retained in the char of lignite which has highly oxygenated functional groups with reducing gas is expected to release $\mathrm{SO}_{2}$ instead of $\mathrm{H}_{2} \mathrm{~S}$, thus it will give benefit in controlling the sulphur gas since it is easier to capture $\mathrm{SO}_{2}$ than $\mathrm{H}_{2} \mathrm{~S}$ with the available technologies. The understanding of the interaction between sulphur and inorganic matter can be used to optimise sulphur retention in the solid phase during pyrolysis. The evolution of $\mathrm{SO}_{2}$ instead of $\mathrm{H}_{2} \mathrm{~S}$ during pyrolysis is also beneficial for the 
Chapter 9

environment in term of pollution control and also for the sulphur capture technology; as mentioned earlier that it is easier to capture $\mathrm{SO}_{2}$ than $\mathrm{H}_{2} \mathrm{~S}$ with the present technology.

To sum up, the present research has improved the understanding of sulphur transformation during lignite pyrolysis, thus it will help device appropriate sulphur control strategies. 


\section{CHAPTER 10}

\section{Conclusions and Recommendations}

\subsection{Conclusions}

\subsubsection{Lignite characterisation}

1. The lignites employed in the present thesis work are categorised as of low quality since they contain very high moisture, high ash, and low fixed carbon, therefore they have a low calorific value. Furthermore, the lignites have a considerable amount of sulphur with different proportions of pyrite, sulphate and organic sulphur.

2. These lignites contain extremely high sodium and chlorine which both of them are water soluble, thus in the form of $\mathrm{NaCl}$.

3. FTIR and solid state ${ }^{13} \mathrm{C}$ NMR spectroscopy studies show that aliphatic carbon presents in significant abundance in the samples. It is confirmed with carbon aromaticity value (fa) obtained for the raw samples, ranging from 0.47-0.62. FTIR and solid state ${ }^{13} \mathrm{C}$ NMR also confirm that the lignites have a high concentration of oxygenated functional structures.

4. Petrographic analysis shows that the raw lignites are dominated by huminite, the precursor of vitrinite. The $\mathrm{Rr}$ values of the raw lignites range between $0.19 \% \mathrm{Rr}$ and $0.27 \% \mathrm{Rr}$. The heat treatment affects the maturation of the sample, it is confirmed with the $\mathrm{Rr}$ of the chars ranges from $1.21 \% \mathrm{Rr}-1.48 \% \mathrm{Rr}$. 


\subsubsection{Pyrite transformation during lignite pyrolysis}

1. The TGA pyrolysis experiments confirmed that during pyrolysis of pyrite alone in nitrogen the pyrite $(\mathrm{S} / \mathrm{Fe}=2)$ transforms to pyrrothite $(1<\mathrm{S} / \mathrm{Fe}<2)$ at ca $720 \mathrm{~K}$ and then to troilite $(\mathrm{S} / \mathrm{Fe}=1)$ at $1200 \mathrm{~K}$. Some of the troilite is further decomposed to elemental iron $(\mathrm{S} / \mathrm{Fe}<1)$ and gaseous elemental sulphur above $1200 \mathrm{~K}$.

2. Twin DTG peaks were observed during pyrolysis of pyrite-lignite blended samples. The first peak was attributed to the devolatilisation of the lignite, while the second peak was related to the pyrite decomposition.

3. Hydrogen sulphide $\left(\mathrm{H}_{2} \mathrm{~S}\right)$ is the major sulphurous gas product released during the pyrolysis of the pyrite-lignite blends, with only a small quantity of sulphur dioxide $\left(\mathrm{SO}_{2}\right)$ as the co-product. However, the inherent pyrite in the lignite with high pyrite content reacts with the oxygenate groups in the lignite to release $\mathrm{SO}_{2}$ as the dominant sulphurous gas product and no hydrogen sulphide during pyrolysis.

4. Inherent inorganic matter in the lignite helps retaining part of the sulphur in the char. The retention of inorganic sulphur in the blended samples was almost constant above $873 \mathrm{~K}$ following complete transformation of pyrite to troilite as confirmed by FTIR, SEM-EDS and XRD analysis.

5. Kinetic parameters were obtained for the lignites, the lignite - pyrite blends and the pyrite, assuming the first-order reactions. The activation energy (E) and frequency factor (A) of the pyrolysis of the lignite samples decrease with increasing temperature over the three temperature regions; at 573-613K E ranges between 107.40-147.56 $\mathrm{kJ} \mathrm{mol}^{-1}$ and A ranges between $1.5 \times 10^{8}-4.9 \times 10^{11} \mathrm{~min}^{-1}$, at $623-753 \mathrm{~K} \mathrm{E}$ ranges between $43.40-64.71 \mathrm{~kJ}^{\mathrm{mol}}{ }^{-1}$ and $\mathrm{A}$ ranges between 
$6.2 \times 10^{2}-2.6 \times 10^{4} \mathrm{~min}^{-1}$, and at 763-1003K E ranges between $15.86-17.0 \mathrm{~kJ} . \mathrm{mol}^{-1}$ and A ranges between 17.0-22.7 $\mathrm{min}^{-1}$, respectively. For the blended samples, the $\mathrm{E}$ and $\mathrm{A}$ values also decrease with increasing temperature, at $573-613 \mathrm{~K} \mathrm{E}$ ranges between $121.84-140.67 \mathrm{~kJ}^{-\mathrm{mol}^{-1}}$ and A ranges between $3.0 \times 10^{9}-1.4 \times 10^{11}$ $\mathrm{min}^{-1}$, at $623-753 \mathrm{~K} \mathrm{E}$ ranges between $55.50-63.81 \mathrm{~kJ} \cdot \mathrm{mol}^{-1}$ and $\mathrm{A}$ ranges between $3.5 \times 10^{3}-2.4 \times 10^{4} \mathrm{~min}^{-1}$, and at $763-1003 \mathrm{~K} \mathrm{E}$ ranges between 24.91$33.50 \mathrm{~kJ} . \mathrm{mol}^{-1}$ and A ranges between 35.7-74.4 $\mathrm{min}^{-1}$, respectively. $\mathrm{E}$ and A obtained for pyrite mineral decomposition between $773-953 \mathrm{~K}$ is $121.60 \mathrm{~kJ} \cdot \mathrm{mol}^{-1}$ and $5.2 \times 10^{6} \mathrm{~min}^{-1}$, respectively. These high $\mathrm{E}$ and $\mathrm{A}$ values are the cause of increasing $\mathrm{E}$ and $\mathrm{A}$ values in the highest temperature region with increasing pyrite content in the blended samples.

\subsubsection{Sulphate transformation during lignite pyrolysis}

1. Pure $\mathrm{CaSO}_{4}$ decomposes at temperatures between $1400-1700 \mathrm{~K}$ in a TGA under nitrogen and a mixture of $50 \%$ nitrogen $+50 \%$ carbon dioxide atmospheres. During pyrolysis of $\mathrm{CaSO}_{4}+\mathrm{L} 1, \mathrm{CaSO}_{4}$ starts to decompose at $623 \mathrm{~K}$ to evolve $\mathrm{SO}_{2} . \mathrm{CaSO}_{4}$ is a stable compound, only a little part of the $\mathrm{CaSO}_{4}$ decomposes. The undecomposed $\mathrm{CaSO}_{4}$ left in the char is still in the form of $\mathrm{CaSO}_{4}$.

2. During thermal decomposition of pure $\mathrm{FeSO}_{4} \cdot 7 \mathrm{H}_{2} \mathrm{O}$ in both nitrogen and a mixture of $50 \%$ nitrogen $+50 \% \mathrm{CO}_{2}$, water is released in temperatures $<543 \mathrm{~K}$, the decomposition of $\mathrm{FeSO}_{4}$ occurs between 813-953 K. $\mathrm{FeSO}_{4}$ is a volatile compound, during pyrolysis of $\mathrm{FeSO}_{4}+\mathrm{L} 1$ almost all $\mathrm{FeSO}_{4}$ is decomposed to release $\mathrm{SO}_{2}$ in the temperature range of $500-800 \mathrm{~K}$.

3. Most of the sulphates in L4 is in the form of iron sulphates which have high volatility and the sulphates start to decompose at $500 \mathrm{~K}$. Sulphates has disappeared from the char produced at $1073 \mathrm{~K}$. 
4. During pyrolysis in the fixed bed reactor in nitrogen at $1073 \mathrm{~K}$, only about $36 \%$ of the total sulphur in the $\mathrm{CaSO}_{4}+\mathrm{L} 1$ decomposes. While in the $\mathrm{FeSO}_{4}+\mathrm{L} 1,88 \%$ of the total sulphur decomposes and about $76 \%$ the sulphur in L4 decomposes. It is also confirmed that $\mathrm{FeSO}_{4}+\mathrm{L} 1$ produces more volatile sulphur than $\mathrm{CaSO}_{4}+\mathrm{L} 1$ during pyrolsysis.

\subsubsection{Organic sulphur transformation during lignite pyrolysis}

1. Both naphthalene thiol in $\mathrm{NT}+\mathrm{L} 1$ and benzyl sulphoxide in $\mathrm{BS}+\mathrm{L} 1$ decompose below $673 \mathrm{~K}$. The retention of total sulphur in $\mathrm{NT}+\mathrm{L} 1$ char decreases significantly from $673 \mathrm{~K}$ to $873 \mathrm{~K}$, increasing pyrolysis temperature to $1073 \mathrm{~K}$ only gives minor reduction on the total sulphur retention in $\mathrm{NT}+\mathrm{L} 1$ char. While the retention of total sulphur in $\mathrm{BS}+\mathrm{L} 1$ reduces significantly from $673 \mathrm{~K}$ to $773 \mathrm{~K}$, above $773 \mathrm{~K}$ the total sulphur retention does not reduce significantly.

2. The retention of total sulphur in L3 and AW L3 reduces significantly from 673 $\mathrm{K}$ to $873 \mathrm{~K}$. Above $873 \mathrm{~K}$ there is not much decrease in the total sulphur retention in L3 and AW L3 chars with increasing pyrolysis temperatures. Above $773 \mathrm{~K}$, the total sample retention in L3 is higher than in AW L3, this is due to the interaction between inorganic matter presents in L3 with the sulphur retaining sulphur in the solid phase.

3. The retention of organic sulphur in L3 and AW L3 is quite similar to the retention of sulphur in $\mathrm{BS}+\mathrm{L} 1$. The organic sulphur retention decreases significantly from 673 to $873 \mathrm{~K}$ and rising the pyrolysis temperature to $1073 \mathrm{~K}$ only give minor effect to the decrease of the organic sulphur in the L3 and AW L3 chars. The retention of organic sulphur in L3 is higher than in AW L3 at the corresponding temperatures due to the interaction between organic sulphur with inorganic matter. Inorganic matter helps to retain organic sulphur in the chars. 
4. FTIR study shows that naphthalene thiol is still observable in the NT+L1 char yielded at $673 \mathrm{~K}$, however at $1073 \mathrm{~K}$ the naphthalene thiol bands has disappeared. SEM micrographs of NT+L1 and its chars as well as BS+L1 and its chars show that the porosity increases by increasing pyrolysis temperatures. This contributes to the decomposition of naphthalene thiol and benzyl sulphoxide.

5. SEM EDS shows that acid washing process dissolves most of the inorganic matter presents in L3. FTIR study shows that sulphur-oxy compounds presents in both L3 and AW L3. The sulphur-oxy compounds are still observable in the char produced at $673 \mathrm{~K}$, but have disappeared in $1073 \mathrm{~K}$ char.

6. Sulphur dioxide is the only gaseous sulphur produced during pyrolysis of AW $\mathrm{L} 3, \mathrm{SO}_{2}$ evolution is from the decomposition of oxygenated organic sulphur and also from the decomposition of other labile organic sulphur which presents in AW L3. The evolution of $\mathrm{SO}_{2}$ induces the released of $\mathrm{CO}_{2}$ and $\mathrm{H}_{2} \mathrm{O}$ which are found to be significant during this lignite pyrolysis.

\subsubsection{Effect of inorganic matter on the sulphur transformation during lignite pyrolysis}

1. The retention of organic and inorganic sulphur in L3 is higher than in AW L3. This is due to the interaction between inherent inorganic matter in L3 with organic sulphur, retaining the organic sulphur in the char. Inorganic matter also reacts with the pyrite decomposition products resulting another inorganic sulphur retained in the char.

2. The retention of organic sulphur L3 is higher than in AW L3 due to the inherent inorganic matter present in the raw lignite which readily reacts with the sulphur to retain sulphur in the char. The decomposition of inorganic sulphur in L3, 
however, is not affected by the inherent inorganic matter; it depends on the type of sulphates present in the sample.

3. Adding kaolinite and sodium to AW L3 affects the sulphur retention during pyrolysis. Sodium has a retention effect on the organic sulphur between 400$500^{\circ} \mathrm{C}$, while kaolinite has a better ability to retain sulphur above $600^{\circ} \mathrm{C}$.

\subsection{Recommendations for Future Research}

Following are some recommendations which were drawn when evaluating the work as presented in Chapter 9:

1. The overall aims of the study of pyrite transformation during lignite pyrolysis have been achieved. In the current study thermal decomposition of pure pyrite was focused on nitrogen atmosphere, slow heating rate (10 and $\left.50 \mathrm{~K} \cdot \mathrm{min}^{-1}\right)$ and with particle size $<150 \mu \mathrm{m}$. For future study it can be extended to use different gas atmospheres, fast heating rates and various ranges of particle sizes. Chemical, physical or spectroscopic analyses which can quantify each type of sulphur in the solid phase (lignite and its char) are also suggested for future work. It is also important to use not only mass spectrometer (MS) but also gas chromatography (GC) to study the sulphur gases evolved during lignite pyrolysis. With those solid and gas sulphur analyses appropriate data for the kinetic study of sulphur transformation can be supplied.

2. For the sulphate transformation during lignite pyrolysis, it is suggested in the future to study the effect of inherent inorganic matter on the sulphate transformation. The work also can be extended to study more wide variety of sulphate model compounds. 
3. It is suggested for future work to study the organic sulphur functionalities in the Australian lignite so that the mechanisms of organic sulphur in lignite can be identified. Other organic sulphur model compounds are also suggested to be studied.

4. It is suggested for future study to perform further analysis of the similarities between added and inherent sulphate as well as added and inherent organic sulphur in lignite samples.

5. The effect of sodium and kaolinite on the sulphur transformation during lignite pyrolysis has been studied. It is recommended to study other forms of inorganic matter on the sulphur transformation in the future study. Chemical and physical analysis to identify the changes of inorganic matter and sulphur forms in the char are valuable to study the mechanism of the reaction between inorganic matter and sulphur during lignite pyrolysis. 


\section{REFERENCES}

1. Australian-Bureau-of-Statistic. Profile of Major Minerals, Oil and Gas. 2006 [cited $200726 \quad$ November 2007]; Available from: http://www.abs.gov.au/Ausstats/abs@.nsf/0/582B60B066B26906CA2570DE00 173BBD?opendocument

2. Telfer, M. and D.K. Zhang, The influence of water-soluble and acid-soluble inorganic matter on sulphur transformations during pyrolysis of low-rank coals. Fuel, 2001. 80(14): p. 2085-2098.

3. Vamvuka, D., S. Troulinos, and E. Kastanaki, The effect of mineral matter on the physical and chemical activation of low rank coal and biomass materials. Fuel, 2006. 85(12-13): p. 1763-1771.

4. Attar, A., Chemistry, thermodynamics and kinetics of reactions of sulphur in coal-gas reactions: A review. Fuel, 1978. 57(4): p. 201-212.

5. Khan, M.R., Prediction of sulphur distribution in products during low temperature coal pyrolysis and gasification. Fuel, 1989. 68(11): p. 1439-1449.

6. Gryglewicz, G., Sulfur transformations during pyrolysis of a high sulfur Polish coking coal. Fuel, 1995. 74(3): p. 356-361.

7. Chen, H., B. Li, and B. Zhang, Decomposition of pyrite and the interaction of pyrite with coal organic matrix in pyrolysis and hydropyrolysis. Fuel, 2000. 79(13): p. 1627-1631.

8. Gryglewicz, G. and S. Jasienko, The behaviour of sulphur forms during pyrolysis of low-rank coal. Fuel, 1992. 71(11): p. 1225-1229.

9. Yan, J., J. Yang, and Z. Liu, SH Radical: The Key Intermediate in Sulfur Transformation during Thermal Processing of Coal. Environ. Sci. Technol., 2005. 39(13): p. 5043-5051.

10. Vuthaluru, H.B., et al., Investigations into the control of agglomeration and defluidisation during fluidised-bed combustion of low-rank coals. Fuel, 1999. 78(4): p. 419-425.

11. Karayigit, A.I. and M. Koksoy, Origin of coal, in Coal Resources, Properties, Utilisation, Pollution, O. Kural, Editor. 1994, Mining Faculty Istanbul Technical University: Istanbul. p. 29-44.

12. Couch, G.R., Lignite resources and characteristics. 1988, IEA Coal Research: London. p. 13.

13. Sandison, C.M., The Organic Geochemistry of Marine-Influenced Coal, in School of Applied Chemistry. 2001, Curtin University of Technology: Perth.

14. AS-2096, Classification and coding systems for Australian coals 1987.

15. ASTM-D-388, Standard Classification of Coals by Rank. 2005.

16. Kural, O., ed. Coal Resources,Properties, Utilization, Pollution. Classification of Coal, ed. A.I. Karayigit and M. Koksoy. 1994, Mining Faculty Istanbul Technical University: Istanbul. 45-58.

17. Ibarra, J.V., E. Munoz, and R. Moliner, FTIR study of the evolution of coal structure during the coalification process. Org. Geochem., 1996. 24(6/7): p. 725-735.

18. Georgakopoulos, A., A. Iordanidis, and V. Kapina, Study of Low Rank Greek Coals Using FTIR Spectroscopy. Energy Sources, 2003. 25: p. 995-1005.

19. Meiler, W. and R. Meusinger, NMR of Coals and Coal Products. Annual Reports on NMR Spectroscopy, 1991. 23: p. 375-406.

20. Ward, C.R., Analysis and significance of mineral matter in coal seams. International Journal of Coal Geology, 2002. 50(1-4): p. 135-168. 
21. Hayashi, J.-I. and C.-Z. Li, Structure and Properties of Victorian Brown Coal, in Advances in the Science of Victorian Brown Coal, C.-Z. Li, Editor. 2004, Elsevier: Amsterdam. p. 11-84.

22. Meyers, R.A., ed. Coal Structure. Mineral Matter in Coal, ed. J.J. Renton. 1982, Academic Press: New York. 283-326.

23. ICCP, The new vitrinite classification (ICCP System 1994). Fuel, 1998. 77(5): p. 349-358.

24. Sykorova, I., et al., Classification of huminite--ICCP System 1994. International Journal of Coal Geology, 2005. 62(1-2): p. 85-106.

25. Finkelman, R.B., Abundance, source, and mode of occurence of the inorganic constituents in coal, in Coal Resources, Properties, Utilization, Pollution, O. Kural, Editor. 1994, Mining Faculty Istanbul Technical University: Istanbul.

26. Ward, C.R., et al., Quantification of mineral matter in the Argonne Premium Coals using interactive Rietveld-based $X$-ray diffraction. International Journal of Coal Geology, 2001. 46(2-4): p. 67-82.

27. Gluskoter, H.J., Electronic low tempearture ashing of bituminous coal. Fuel, 1965. 44: p. 285-291.

28. Zhang, L., A. Sato, and Y. Ninomiya, CCSEM analysis of ash from combustion of coal added with limestone. Fuel, 2002. 81(11-12): p. 1499-1508.

29. Painter, P.C., et al., Fourier Transform Infrared study of mineral matter in coal. A novel method for quantitative mineralogical analysis. Fuel, 1978. 57(6): p. 337-344.

30. Barooah, P.K. and M.K. Baruah, Sulphur in Assam coal. Fuel Processing Technology, 1996. 46(2): p. 83-97.

31. Calkins, W.H., The chemical forms of sulfur in coal: a review. Fuel, 1994. 73(4): p. 475-484.

32. Miura, K., et al., Analysis of formation rates of sulfur-containing gases during the pyrolysis of various coals. Energy \& Fuels, 2001. 15: p. 629-636.

33. Spears, D.A., ed. Pyrite in some UK Coals. Processing and Utilization of HighSulfur Coals IV, ed. P.R. Dugan, D.R. Quigley, and Y.A. Attia. 1991, Elsevier Science Publishers B.V.: Amsterdam.

34. Berner, R.A., Sedimentary pyrite formation : An update. Geochimica et Cosmochimica Acta, 1984. 48: p. 605-615.

35. Perry, R.H. and C.H. Chilton, Chemical Engineers' Handbook. 5th. ed. 1974.

36. Davidson, R.M., Organic Sulphur in Coal, in IEA Coal Research. 1993, IEA Coal Research: London. p. 13.

37. Ryan, B. and A. Ledda, A review of sulphur in coal : with specific reference to Telkwa deposit, North-Western British Columbia. Geological Fieldwork 1997, 1998. 1.

38. Mullens, S., et al., A study of the reductive pyrolysis behaviour of sulphur model compounds. Journal of Analytical and Applied Pyrolysis, 2003. 70(2): p. 469491.

39. Li, C.-Z., Conversion of coal- $N$ and coal-S during pyrolysis, gasification and combustion, in Advances in the Science of Victorian Brown Coal ,C.-Z. Li, Editor. 2004, Elsevier: Amsterdam. p. 286-350.

40. Calkins, W.H., Investigation of Organic Sulfur-Containing Structures in Coal by Flash Pyrolysis Experiments. Energy \& Fuels, 1987. 1: p. 59-64.

41. ASTM-D-2492, Standard Test Method for Forms of Sulphur in Coal. 1998.

42. ASTM-D-3177, Standard Test Methods for Total Sulfur in the Analysis Sample of Coal and Coke. 2002.

43. Guo, Z., Z. Fu, and S. Wang, Sulfur distribution in coke and sulfur removal during pyrolysis. Fuel Processing Technology, 2007. 88(10): p. 935-941. 
44. Davidson, R.M., Quantifying organic sulfur in coal. Fuel, 1994. 73: p. 9881005.

45. Olivella, M.A., et al., A study of sulfur functionalities in fossil fuels using destructive- (ASTM and Py-GC-MS) and non-destructive- (SEM-EDX, XANES and XPS) techniques. Fuel, 2002. 81(4): p. 405-411.

46. Stefanova, M., et al., Reductive pyrolysis of Miocene-aged lignite lithotypes using MS and GC/MS detection systems for analysis of organic sulphur groups. Fuel, 2005. 84(1): p. 71-79.

47. Maes, I.I., et al., Sulfur functionalities and physical characteristics of the Maritza Iztok Basin lignite. Fuel, 1996. 75(11): p. 1286-1293.

48. Gorbaty, M.L., G.N. George, and S.R. Kelemen, Chemistry of organically bound sulphur forms during the mild oxidation of coal. Fuel, 1990. 69: p. 1065-1067.

49. Gorbaty, M.L. and S.R. Kelemen, Characterization and reactivity of organically bound sulfur and nitrogen fossil fuels. Fuel Processing Technology, 2001. 71(13): p. 71-78.

50. Gorbaty, M.L., et al., Characterization and thermal reactivity of oxidized organic sulphur forms in coals. Fuel, 1992. 71(11): p. 1255-1264.

51. Hu, G., et al., Decomposition and oxidation of pyrite. Progress in Energy and Combustion Science, 2006. 32(3): p. 295-314.

52. Kullerud, G. and H. Yoder, Pyrite stability relations in the Fe-S system. Econ Geol, 1959. 54: p. 533-572.

53. Sack, R.O. and D.S. Ebel, Thermochemistry of Sulfide Mineral Solutions. Review in Mineralogy \& Geochemistry, 2006. 61: p. 265-364.

54. Cypres, R., M. Ghodsi, and R. Stocq, Behaviour of pyrite during hydrogenation of graphite at atmospheric pressure. Fuel, 1981. 60(3): p. 247-250.

55. Maa, P.S., C. Randall Lewis, and J. Hamrin, Charles E., Sulphur transformation and removal for Western Kentucky coals. Fuel, 1975. 54(1): p. 62-69.

56. Lambert, J.M., G. Simkovich, and P.L. Walker, The kinetics and mechanism of the pyrite-to-pyrrothite transformation. Metalurgical and Materials Transaction B, 1998. 29B: p. 385-396.

57. Boyabat, N., et al., Thermal decomposition of pyrite in the nitrogen atmosphere. Fuel Processing Technology, 2004. 85(2-3): p. 179-188.

58. Borah, D., M. Barua, and M.K. Baruah, Dependence of pyrite concentration on kinetics and thermodynamics of coal pyrolysis in non-isothermal systems. Fuel Processing Technology, 2005. 86(9): p. 977-993.

59. Ericsson, I. and P. Almen, Kinetic and quantitative studies of the formation of SO2 from iron(II) and calcium sulfates by sequential and fractionated pyrolysis. Journal of Analytical and Applied Pyrolysis, 1996. 36(1): p. 37-49.

60. Ibarra, J.V., A.J. Bonet, and R. Moliner, Release of volatile sulfur compounds during low temperature pyrolysis of coal. Fuel, 1994. 73(6): p. 933-939.

61. Telfer, M.A. and D.K. Zhang, Investigation of Sulfur Retention and the Effect of Inorganic Matter during Pyrolysis of South Australian Low-Rank Coals. Energy Fuels, 1998. 12(6): p. 1135-1141.

62. Xu, L., et al., Behavior of organic sulfur model compounds in pyrolysis under coal-like environment. Fuel Processing Technology, 2004. 85(8-10): p. 10131024.

63. Yperman, J., et al., Determination of sulfur groups in pyrolysed low-rank coal by atmospheric-pressure t.p.r. Fuel, 1995. 74(9): p. 1261-1266.

64. Telfer, M., Sulphur transformations during pyrolysis of Low-rank coals and characterisation of Ca-based sorbents, in Department of Chemical Engineering. 1999, University of Adelaide: Adelaide. p. 323. 
65. Li, C.-Z., ed. Advances in the science of Victorian brown coal. Pyrolysis of Victorian brown coal, ed. J.-i. Hayashi and K. Miura. 2004, Elsevier: Amsterdam. 134-222.

66. Schafer, H.N.S., Pyrolysis of brown coals. 1. Decomposition of acid groups in coals containing carboxyl groups in the acid and cation forms. Fuel, 1979. 58(9): p. 667-672.

67. Sathe, C., Y. Pang, and C.Z. Li, Effects of Heating Rate and Ion-Exchangeable Cations on the Pyrolysis Yields from a Victorian Brown Coal. Energy Fuels, 1999. 13(3): p. 748-755.

68. Manzoori, A.R. and P.K. Agarwal, The fate of organically bound inorganic elements and sodium chloride during fluidized bed combustion of high sodium, high sulphur low rank coals. Fuel, 1992. 71(5): p. 513-522.

69. Quyn, D.M., H. Wu, and C.-Z. Li, Volatilisation and catalytic effects of alkali and alkaline earth metallic species during the pyrolysis and gasification of Victorian brown coal. Part I. Volatilisation of $\mathrm{Na}$ and $\mathrm{Cl}$ from a set of $\mathrm{NaCl}$ loaded samples. Fuel, 2002. 81(2): p. 143-149.

70. Hayashi, J.-i. and K. Miura, eds. Pyrolysis of Victorian brown coal. Advances in the science of Victorian brown coal, ed. C.-Z. Li. 2004, Elsevier: Amsterdam. 134-222.

71. Cheng, J., et al., Sulfur removal at high temperature during coal combustion in furnaces: a review. Progress in Energy and Combustion Science, 2003. 29(5): p. 381-405.

72. Yan, R., et al., Thermodynamic study of the behaviour of minor coal elements and their affinities to sulphur during coal combustion. Fuel, 1999. 78(15): p. 1817-1829.

73. Anthony, E.J. and D.L. Granatstein, Sulfation phenomena in fluidized bed combustion systems. Progress in Energy and Combustion Science, 2001. 27(2): p. 215-236.

74. Ninomiya, Y., et al., Combustion and De-SO $\mathrm{S}_{x}$ behavior of high-sulfur coals added with calcium produced from biomass pyroligneous acid. Fuel, 2004. 83(16): p. 2123-2131.

75. Solomon, P.R., M.A. Serio, and E.M. Suuberg, Coal pyrolysis: Experiments, kinetic rates and mechanisms. Progress in Energy and Combustion Science, 1992. 18(2): p. 133-220.

76. Friedman, H.L., J. Polymer Sci., 1963. Part C(6): p. 183.

77. Coats, A.W. and J.P. Redfern, Kinetic parameters from thermogravimetric data. Nature, 1964. 201: p. 68-69.

78. Garcia-Labiano, F., E. Hampartsoumian, and A. Williams, Determination of sulfur release and its kinetics in rapid pyrolysis of coal. Fuel, 1995. 74(7): p. 1072-1079.

79. Sugawara, K., et al., Effect of heating rate and temperature on pyrolysis desulfurization of a bituminous coal. Fuel Processing Technology, 1994. 37(1): p. 73-85.

80. Yip, K., H. Wu, and D.k. Zhang, Effect of Inherent Moisture in Collie Coal during Pyrolysis Due to in-Situ Steam Gasification. Energy Fuels, 2007. 21(5): p. 2883-2891.

81. Cole, D.I. and D.L. Roberts, Lignite from the western coastal plain of South Africa. Journal of African Earth Sciences, 1996. 23(1): p. 95-117.

82. GriffinCoal. Measuring coal quality. 2006 [cited 200624 August]; Available from: http://www.griffincoal.com.au.

83. Grigore, M., et al., Mineral matter in coals and their reactions during coking. International Journal of Coal Geology, 2008: p. doi :10.1016/j.coal.2008.08.03. 
84. Yudovich, Y.E. and M.P. Ketris, Chlorine in coal : A review. International Journal of Coal Geology, 2006. 67: p. 127-144.

85. Gavlak, R., et al., Soil, Plant and Water Reference Methods for the Western Region. 2nd ed. WCC-123. 2003: WREP-125. 1-5.

86. Ibarra, J.V., E. Munoz, and R. Moliner, FTIR study of evolution of coal structure during the coalification process. Org. Geochem, 1996. 24(6/7): p. 725-735.

87. Cerny, J., Structural dependence of $\mathrm{CH}$ bond absorptivities and consequences for FT-i.r. analysis of coals. Fuel, 1996. 75(11): p. 1301-1306.

88. Walker, R. and M. Mastalerz, Functional group and individual maceral chemistry of high volatile bituminous coals from southern Indiana: controls on coking. International Journal of Coal Geology, 2004. 58(3): p. 181-191.

89. Descostes, M., et al., Effect of carbonate ions on pyrite $\left(\mathrm{FeS}_{2}\right)$ dissolution. Bull. Soc. geol. France, 2002. 173(3): p. 265-270.

90. Meiler, W. and R. Meusinger, eds. Application of NMR Methods in Coal Research. Annual reports on NMR spectroscopy ed. G.A. Webb. Vol. 23. 1991, Academic Press: London. 331-361.

91. Miknis, F.P., et al., Cross-polarization magic-angle spinning 13C NMR spectra of coals of varying rank. Organic Geochemistry, 1981. 3(1-2): p. 19-28.

92. Saito, K., K. Kanehashi, and I. Komaki, Applications of NMR Techniques to Coal Science. Annual Reports on NMR Spectroscopy. 44: p. 23-74.

93. Monsef-Mirzai, P., et al., Measurement of -OH groups in coals of different rank using microwave methodology, and the development of quantitative solid state n.m.r. methods for in situ analysis. Fuel, 1995. 74(5): p. 674-683.

94. Snape, C.E., et al., Quantitative reliability of aromaticity and related measurements on coals by 13C n.m.r. A debate. Fuel, 1989. 68(5): p. 547-548.

95. Wind, R.A., et al., An investigation of coal by means of e.s.r., 1 H n.m.r., $13 \mathrm{C}$ n.m.r. and dynamic nuclear polarization. Fuel, 1987. 66(7): p. 876-885.

96. Smernik, R.J., L. Schwark, and M.W.I. Schmidt, Assessing the quantitative reliabilitybof solid state ${ }^{13} \mathrm{C} N \mathrm{NR}$ spectra of kerogens across a gradient of thermal maturity. Solid Stae Nuclear Magnetic Resonance 2006. 29: p. 312-321.

97. Dereppe, J.-M. and C. Moreaux, A limitation of ${ }^{13} \mathrm{C}$ CP-MAS n.m.r. spectroscopy for the study of treated coals. Fuel, 1987. 66: p. 1088-1089.

98. MacPhee, J.A. and B.N. Nandi, ${ }^{13}$ C n.m.r as a probe for the characterisation of the low-temperature oxidation of coal. Fuel, 1981. 60: p. 169-170.

99. Kortenski, J. and A. Sotirov, Petrography of the Neogene lignite from the Sofia basin, Bulgaria. International Journal of Coal Geology, 2004. 57: p. 117-126.

100. Liu, Y., D. Che, and T. Xu, Catalytic reduction of SO2 during combustion of typical Chinese coals. Fuel Processing Technology, 2002. 79(2): p. 157-169.

101. Chen, H.-k., et al., Transformation of sulfur during pyrolysis and hydropyrolysis of coal. Fuel, 1998. 77(6): p. 487-493.

102. Guin, J.A., et al., Pyrite-Catalyzed Hydrogenolysis of Benzothiophene at Coal Liquefaction Conditions. Ind. Eng. Chem. Process Des. Dev., 1980. 19: p. 440446.

103. Yip, K., H. Wu, and D.-k. Zhang. Mathematical Modelling of Collie Coal Pyrolysis Considering the Effect of Steam Produced in situ from Coal Inherent Moisture and Pyrolytic Water. in The $32^{\text {nd }}$ International Symposium on Combustion. 2008. Montreal, Canada.

104. Kizgut, S. and S. Yilmaz, Characterization and non-isothermal decomposition kinetics of some Turkish bituminous coals by thermal analysis. Fuel Processing Technology, 2004. 85(2-3): p. 103-111.

105. Liu, Q., et al., Effect of inorganic matter on reactivity and kinetics of coal pyrolysis. Fuel, 2004. 83(6): p. 713-718. 
106. Elder, J.P. and M.B. Harris, Thermogravimetry and differential scanning calorimetry of Kentucky bituminous coals. Fuel, 1984. 63(2): p. 262-267.

107. Ceylan, K., H. Karaca, and Y. Onal, Thermogravimetric analysis of pretreated Turkish lignites. Fuel, 1999. 78(9): p. 1109-1116.

108. Lambert, J.J.M., G. Simkovich, and J.P.L. Walker, Production of pyrrhotites by pyrite reduction. Fuel, 1980. 59(10): p. 687-690.

109. Yani, S. and D.K. Zhang, Transformation of organic and inorganic sulphur in a lignite during pyrolysis : Influence of inherent and added inorganic matter. Proc. Combust. Inst., 2009. 32: p. 2083-2089.

110. Ibarra, J., et al., Influence of weathering on the sulphur removal from coal by pyrolysis. Fuel Processing Technology, 1989. 21(1): p. 63-73.

111. Ahmaruzzaman, M. and D.K. Sharma, Non-isothermal kinetic studies on coprocessing of vacuum residue, plastics, coal and petrocrop. Journal of Analytical and Applied Pyrolysis, 2005. 73(2): p. 263-275.

112. Medvedev, K.P. and V.M. Petropolskaya, Volatility of the different forms of sulphate sulphur in the carbonisation of coals. UKhIN, 1966: p. 10-13.

113. Aquilar-Santilan, J., Wetting of $\mathrm{Al}_{2} \mathrm{O}_{3}$ by molten aluminium : the influence of $\mathrm{BaSO}_{4}$ additions. Journal of Nanomaterials, 2008. vol. 2008(Article ID 629185): p. 12 pages. doi : 10.1155/2008/629185.

114. Stern, K.H., High temperature properties and thermal decomposition of inorganic salts with oxyanions. Kindle Edition ed. 2000: CRC; 1 edition. 288.

115. Raask, E., Mineral Impurities in Coal Combustion. 1985: Hemisphere Publishing. 293-312.

116. Vuthaluru, H.B. and D. Zhang, Control methods for remediation of ash-related problems in fluidised-bed combustors. Fuel Processing Technology, 1999: p. 145-156.

117. Vuthaluru, H.B. and D.K. Zhang, Effect of Ca- and Mg-bearing minerals on particle agglomeration defluidisation during fluidised-bed combustion of a South Australian lignite. Fuel Processing Technology, 2001. 69: p. 13-27.

118. Czaplicki, A. and W. Smolka, Sulfur distribution within coal pyrolysis products. Fuel Processing Technology, 1998. 55(1): p. 1-11.

119. Garcia-Labiano, F., et al., Sulfur release during the devolatilization of large coal particles. Fuel, 1996. 75(5): p. 585-590.

120. Hu, H., et al., Product distribution and sulfur behavior in coal pyrolysis. Fuel Processing Technology, 2004. 85(8-10): p. 849-861.

121. Hu, G., et al., Review of the direct sulfation reaction of limestone. Progress in Energy and Combustion Science, 2006. 32: p. 386-407.

122. Coates, J., ed. Interpretation of Infrared Spectra, a Practical Approach. Encyclopedia of Analytical Chemistry, ed. R.A. Meyers. 2000, John Wiley \& Sons Ltd: Chichesters. 1-23.

123. Liu, K., et al., Desulphurization of coal via low temperature atmospheric alkaline oxidation. Chemosphere, 2008. 71: p. 183-188.

124. Stock, L.M., R. Wolny, and B. Bal, Sulfur distribution in American Bituminous Coals. Energy \& Fuels, 1989. 3(6): p. 651-661.

125. Yperman, J., et al., Sulphur group analysis in solid matrices by atmospheric pressure-temperature programmed reduction. Analytica Chimica Acta, 1999. 395(1-2): p. 143-155.

126. Uzun, D. and S. Ozdogan, Sulfur removal from original and acid treated lignites by pyrolysis. Fuel, 2006. 85(3): p. 315-322.

127. Qi, Y., et al., Sulfur release from coal in fluidized-bed reactor through pyrolysis and partial oxidation with low concentration of oxygen. Fuel, 2004. 83(16): p. 2189-2194. 
128. Borah, D., Desulphurization of organic sulphur from coal by electron transfer process with Co2+ ion. Fuel Processing Technology, 2005. 86(5): p. 509-522.

129. Silverstein, R.M., G.C. Bassler, and T.C. Morril, Spectrometric identification of organic compounds. 1991, New York: Wiley.

130. Song, Z., et al., Hydrous pyrolysis transformation of organic sulfur compounds : Part 1. Reactivity and chemical changes. Org. Geochem., 2005. 36: p. 15231532.

131. Arenillas, A., et al., Characterisation of model compounds and a synthetic coal by TG/MS/FTIR to represent the pyrolysis behaviour of coal. Journal of Analytical and Applied Pyrolysis, 2004. 71(2): p. 747-763.

132. Li, X., et al., A study on combustion of Chinese coals by TA/MS. Journal of Analytical and Applied Pyrolysis, 2003. 67(2): p. 393-406.

133. Gaffney, J.S. and N.A. Marley, The impacts of combustion emission on air quality and climate - From coal to biofuels and beyond. Atmospheric Environment, 2009. 43: p. 23-36.

134. Li, C.-Z., Some recent advances in the understanding of the pyrolysis and gasification behaviour of Victorian brown coal. Fuel, 2007. 86(12-13): p. 16641683.

135. Querol, X., et al., Seasonal evolution of suspended particles around a large coal-fired power station : particulate levels and source. Atmospheric Environment 1998. 32: p. 1963-1978.

136. Liu, Q., et al., Effect of mineral on sulfur behavior during pressurized coal pyrolysis. Fuel Processing Technology, 2004. 85(8-10): p. 863-871. 


\section{APPENDICES}

\section{Appendix A FTIR Spectra}

FTIR spectra of 9 lignite samples, namely, L3 to L11, respectively and their chars produced at $773 \mathrm{~K}$ to complement FTIR spectra presented in Chapter 4 are presented in this Appendix.

\section{A.1 FTIR spectra of Australian lignites}

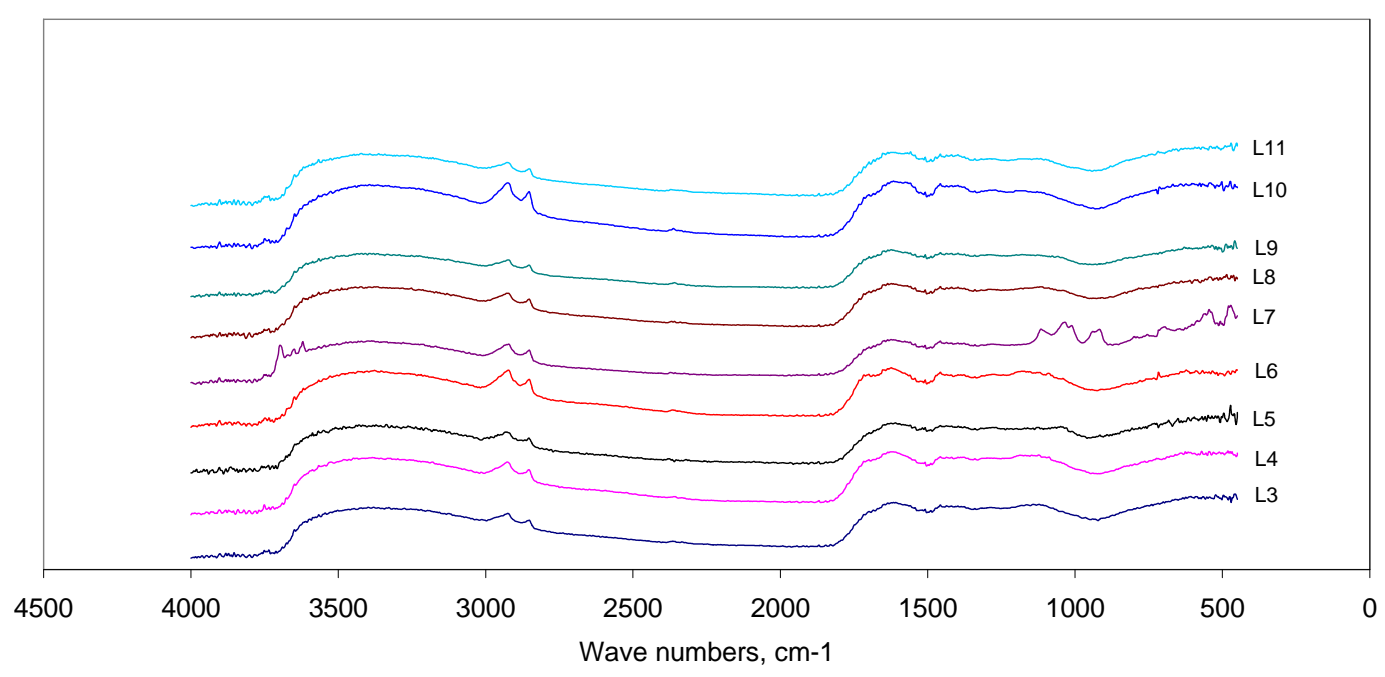

Figure A.1 FTIR spectra of L3 to L11, respectively 


\section{A.2 FTIR of chars produced at $773 \mathrm{~K}$}

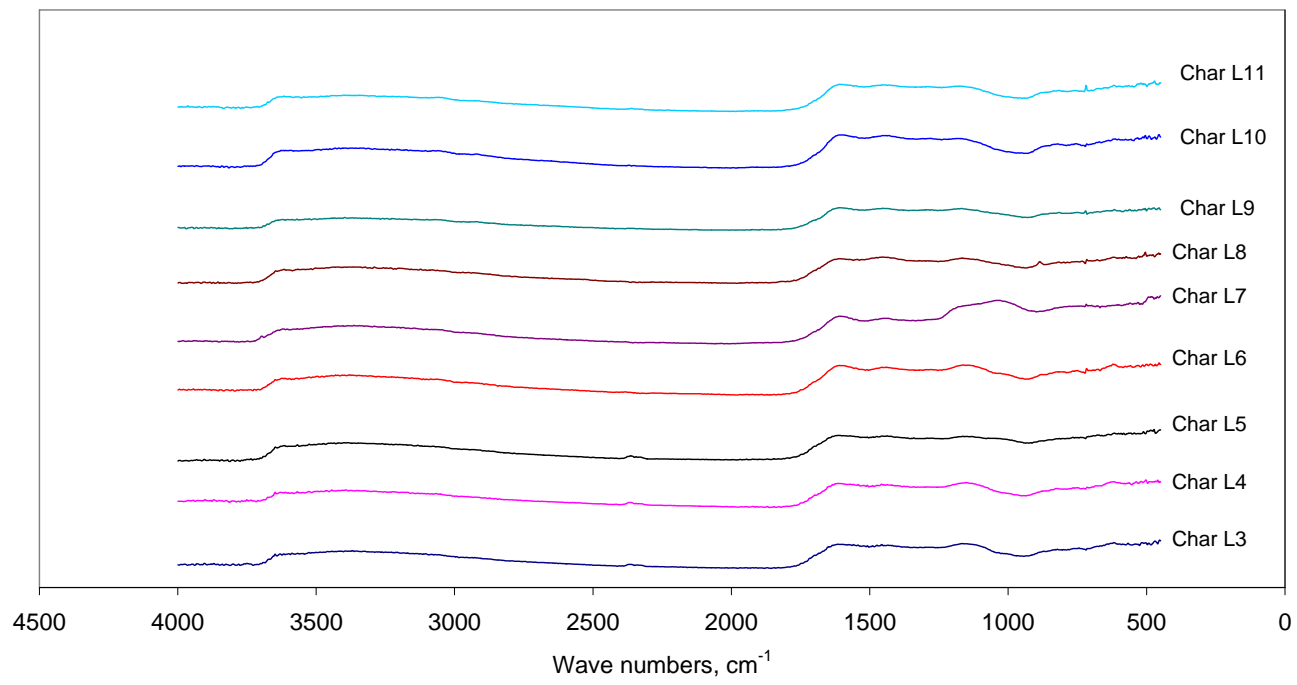

Figure A.2 FTIR spectra of L3-L11 chars produced at $773 \mathrm{~K}$ 


\section{Appendix B NMR Spectra}

\section{B.1 Solid state ${ }^{13} \mathrm{C}$ NMR spectra of selected lignites}

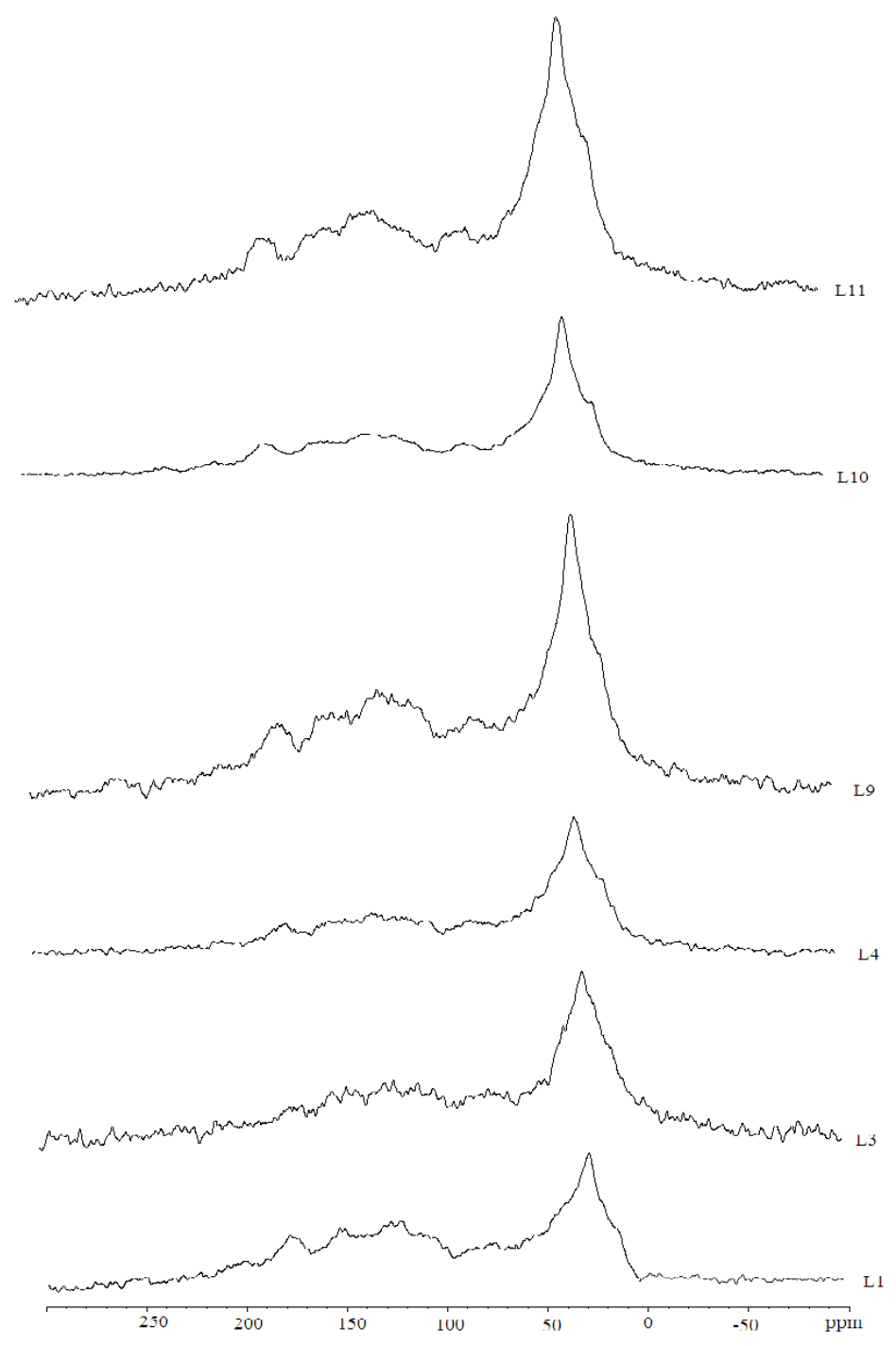

Figure B.1 Solid state ${ }^{13}$ C NMR spectra of L1, L3, L4, L9, L10 and L11, respectively 


\section{B.2 Solid state ${ }^{13} \mathrm{C}$ NMR spectra of selected chars at $773 \mathrm{~K}$}
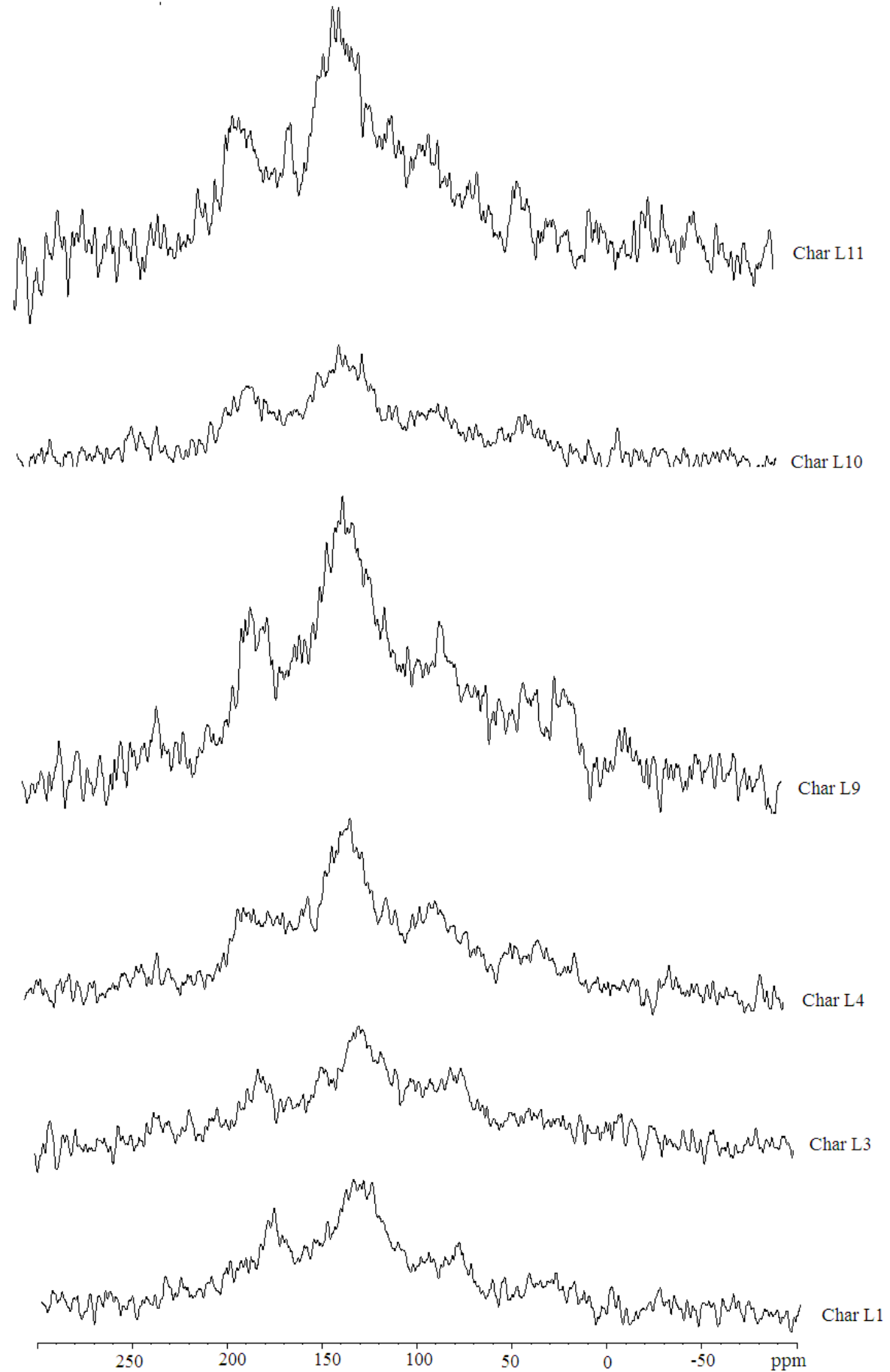

Figure B.2 Solid state ${ }^{13} \mathrm{C}$ NMR spectra of lignite chars produced at $773 \mathrm{~K}$ 
Appendices

\section{Appendix C XRD spectra of lignite Ashes}

Additional XRD diffractograms of high temperature ash (HTA) of lignites prepared at three different temperatures, namely, 723, 823 and $923 \mathrm{~K}$, respectively as those presented in Chapter 4 are presented in this appendix .

\section{C.1 HTA of lignites at $723 \mathrm{~K}$}

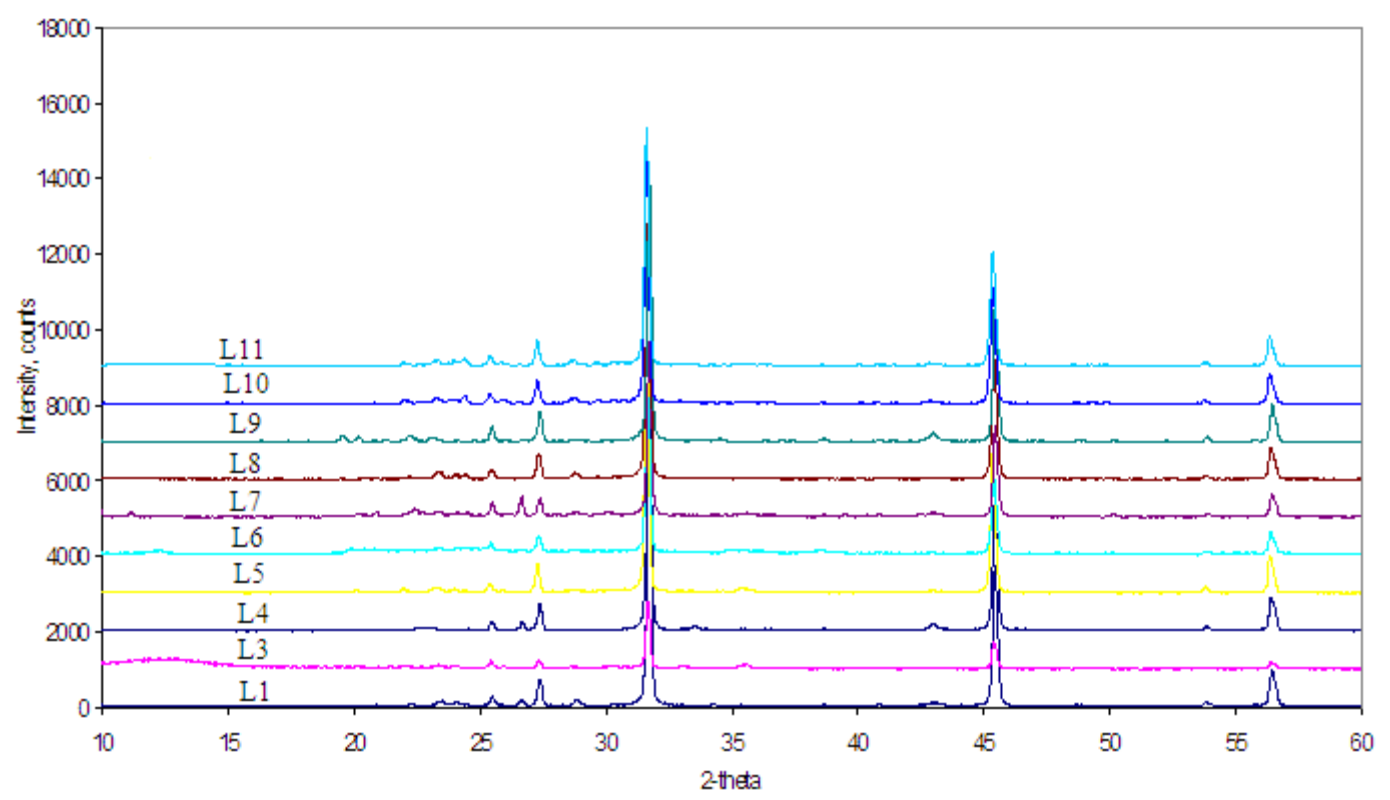

Figure C.1 X-ray diffractogram of lignite ash prepared at $723 \mathrm{~K}$ 


\section{C2. HTA of lignites at $823 \mathrm{~K}$}

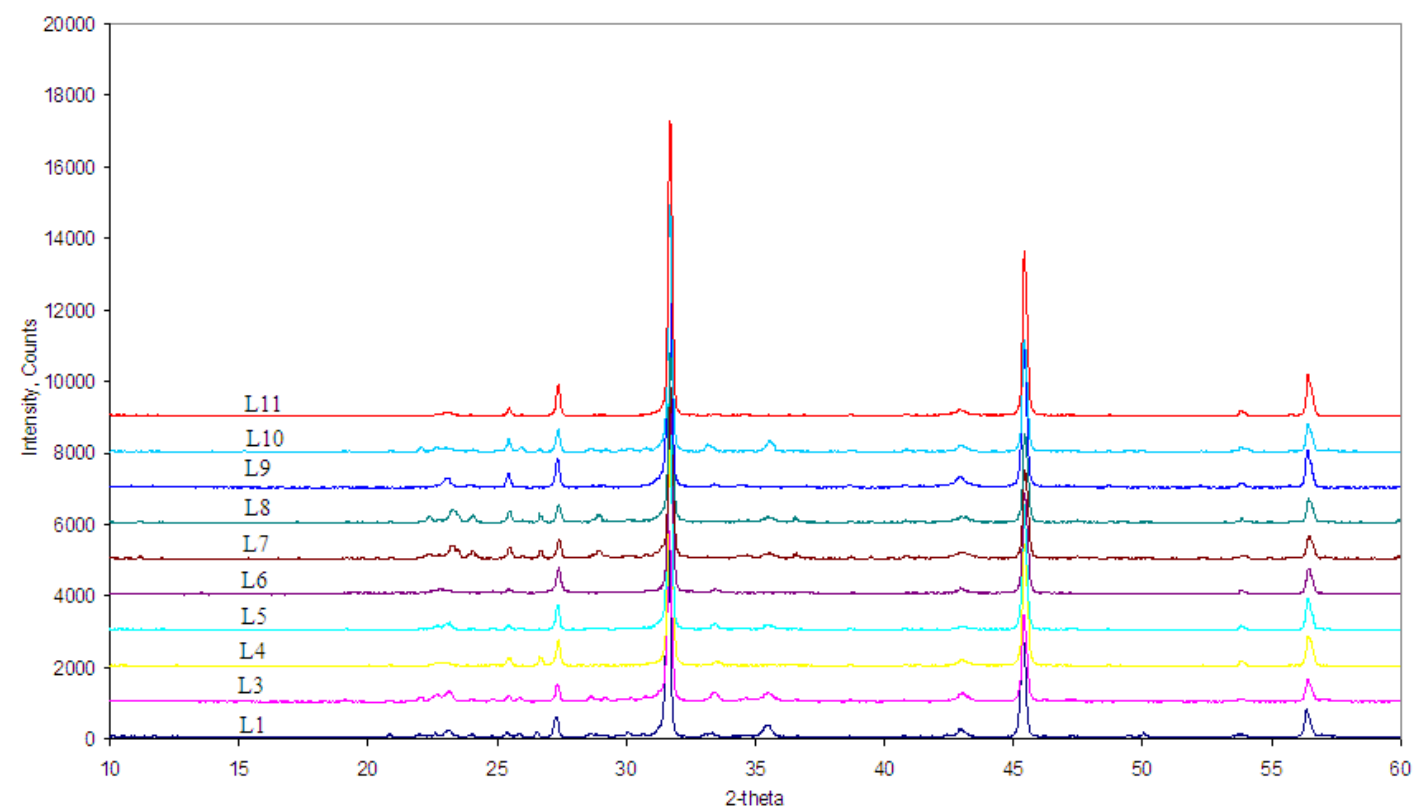

Figure C.2 X-ray diffractogram of lignite ash prepared at $823 \mathrm{~K}$

\section{C3. HTA of lignites at $923 \mathrm{~K}$}

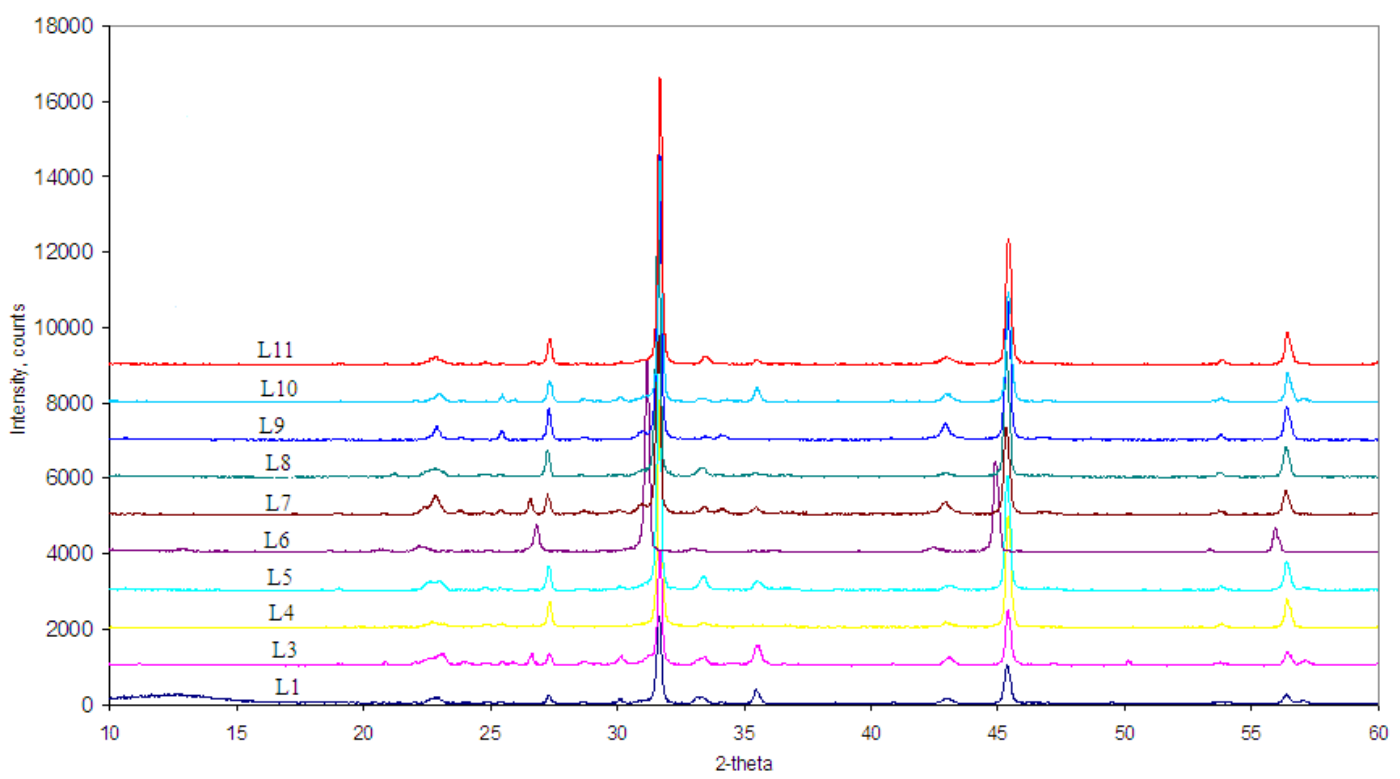

Figure C.3 X-ray diffractogram of lignite ash prepared at $923 \mathrm{~K}$ 
